\title{
Driven Motion and Instability of an Atmospheric Pressure Arc
}

\author{
Max Karasik
}

A Dissertation

Presented to the Faculty

of Princeton University

in Candidacy for the Degree

of Doctor of Philosophy

ReCommended for AcCeptance by the

Department of Astrophysical Sciences

January 2000 
(C) Copyright by Max Karasik, 1999. All rights reserved. 


\section{Abstract}

Atmospheric pressure arcs are used extensively in applications such as welding and metallurgy. However, comparatively little is known of the physics of such arcs in external magnetic fields and the mechanisms of the instabilities present. In order to address questions of equilibrium and stability of such arcs, an experimental arc furnace is constructed and operated in air with graphite cathode and steel anode at currents 100-250 A. The arc is diagnosed with a gated intensified camera and a collimated photodiode array, as well as fast voltage and current probes.

Experiments are carried out on the response of the arc to applied transverse $\mathrm{DC}$ and $\mathrm{AC}$ (up to $\approx 1 \mathrm{kHz}$ ) magnetic fields. The arc is found to deflect parabolically for DC field and assumes a growing sinusoidal structure for AC field. A simple analytic two-parameter fluid model of the arc dynamics is derived, in which the inertia of the magnetically pumped cathode jet balances the applied $\vec{J} \times \vec{B}$ force. Time variation of the applied field allows evaluation of the parameters individually. A fit of the model to the experimental data gives a value for the average jet speed an order of magnitude below Maecker's estimate of the maximum jet speed.

A spontaneous instability of the same arc is investigated experimentally and modeled analytically. The presence of the instability is found to depend critically on cathode dimensions. For cylindrical cathodes, instability occurs only for a narrow range of cathode diameters. Cathode spot motion is proposed as the mechanism of the instability. A simple fluid model combining the effect of the cathode spot motion and the inertia of the cathode jet successfully describes the arc shape during low amplitude instability. The amplitude of cathode spot motion required by the model is in agreement with measurements. The average jet velocity required is ap- 
proximately equal to that inferred from the transverse magnetic field experiments. Reasons for spot motion and for cathode geometry dependence are discussed.

An exploratory study of the instability of the arc in applied axial magnetic field is also described. Applicability of the results of the thesis to an industrial steelmaking furnace is considered. 
Dedicated to my parents,

Isak and Natalie,

and to my grandparents,

Stashek and Nina. 


\section{Acknowledgments}

I would like first of all to thank my thesis advisor, Stewart Zweben. What seemed at first to be a well-studied, very applied problem, through his guidance turned out to be a challenging and scientifically rewarding basic physics research experience. His physical insight and experience have been invaluable guides for this work. His dedication and integrity have been a great example and an inspiration.

I am very thankful to Lane Roquemore for helping get the Arc Furnace off the ground and for his contributions to the project. I would like to thank Paul Bellan for introducing the subject to my advisor and for enlightening discussions and Glen Wurden for loaning the LANL fast camera. I thank Leonid Zakharov for his physics guidance and interest in the project, and Sam Cohen, G. Bendzak, Ben Bowman, J. Heberlein, and S.-E. Stenkvist for fruitful discussions on arc physics. The support of Dale Meade, Lewis Meixler and Ken Young is gratefuly acknowledged.

Deep thanks go to Manfred Bitter and Schweickh von Goeler for help with German literature. I would also like to thank Yevgeny Raitses and Vlad Savchenko for many helpful discussions and suggestions.

I learned a great deal from Erick May and Olufemi Dosumnu who did their summer projects on the Arc Furnace.

I would like to thank Dick Yager for lots of technical assistance, expert advice, and know-how, Tom Holoman for great help in constructing the Arc Furnace, Greg Lemunyan for lots of help with cameras and video acquisition, John Robinson for much help with data acquisition hardware and software, Troy Carter for his computer expertise, as well as George Renda for assistance with photodiodes. 
I am very grateful to Prof. Ron Davidson and Phil Efthimion for reading the thesis and suggesting improvements.

This work was supported by Department of Energy contract \# DE-AC02-76CH03073.

I am glad to have had Sam Cohen as my faculty advisor and had learned a great deal in his lab course. I would like to thank my my theory project advisor Morell Chance. Many thanks to my experimental project supervisor Ira Lehrman for teaching me electron accelerators, RF cavities and soldering.

I am very thankful to PPL librarians Luan Huang, Sharon Brown, and Mitchell Brown for helping with so many literature requests and for digging up 40-year-old sources and lab reports.

I would like to thank Barbara Sarfaty for taking care of us in the department and keeping us well-insulated from the bureaucracy, and Terry Greenberg for a much needed smile in tough times.

Princeton was a much better place with friends like Phil Snyder, Ed Chao, Vlad Savchenko, Scott Hsu, Bob Heeter, Troy Carter, Chris Harrison, Bryan Fong, Dmitri Uzdensky, Stas Boldyrev, and John Wright.

I would like to thank Master Mikhail for introducing me to the art of Tae Kwon Do. I thank Prof. Erika Gilson for making Turkish logical and fun to learn.

I am very grateful to Nelly, Mikhail, Mark, and Steven Karasik for all they have done for me.

My warmest thanks go to my parents, Isak and Natalie, my sister Julie, and to my grandparents, Stashek and Nina, for all their love and support.

I thank my alma mater, The Cooper Union.

I thank Şuhnaz for providing the antidote and for making these years the best.

November 30, 1999 


\section{Table of Contents}

$\begin{array}{ll}\text { Abstract } & \text { iii }\end{array}$

1 Introduction 1

1.1 Uses of Atmospheric Pressure Arcs . . . . . . . . . . . . . . 1

1.2 Objectives of this Thesis $\ldots \ldots \ldots \ldots$

1.3 An Overview of the Physics of Atmospheric Pressure Arcs . . . . 4

1.3 .1 Arc Structure . . . . . . . . . . . . . . . . . 4

1.3.2 Physics of the cathode jet . . . . . . . . . . 7

1.4 An Overview of Previous Work . . . . . . . . . . . . . . 9

1.4.1 Arc deflection . . . . . . . . . . . . . . . 9

1.4 .2 Arc instability . . . . . . . . . . . . . . . 10

1.4 .3 Summary . . . . . . . . . . . . . . . . . . 12

1.5 Similarities and Differences of the PPPL Arc and the Industrial Furnace Arcs . . . . . . . . . . . . . . . . . . . . . 14

1.6 Outline of the Thesis . . . . . . . . . . . . . 15

2 Experimental Apparatus 17

2.1 Arc Furnace Hardware . . . . . . . . . . . . . . . . . 17

2.1.1 Cathode position control . . . . . . . . . . . . . 22

2.1.2 Transverse magnetic field experiments . . . . . . . . 22

2.1.3 Arc stability experiments . . . . . . . . . . . . . 24 
2.1.4 Safety practices in arc furnace operation . . . . . . . . 26

2.2 Diagnostics . . . . . . . . . . . . . . . . . . . . 27

2.2.1 Arc voltage and current monitoring . . . . . . . . . . 27

2.2.2 Monitoring of the applied magnetic field . . . . . . . . . 27

2.2.3 Uncollimated light monitor . . . . . . . . . . . . . . . 27

2.2.4 Gated imaging of the arc . . . . . . . . . . . . . 28

2.2.5 Continuous arc position monitoring . . . . . . . . . . . 29

2.3 Data Acquisition . . . . . . . . . . . . . . . . . . 30

2.4 Arc characteristics . . . . . . . . . . . . . . . . 31

2.4.1 Static characteristics . . . . . . . . . . . . . . 31

2.4.2 Conductivity temperature of the arc plasma . . . . . . . 32

2.4.3 Dynamic characteristics . . . . . . . . . . . . . . . 33

3 Arc Deflection in Transverse Magnetic Field 43

3.1 Experimental Setup . . . . . . . . . . . . . . . . . . . 43

3.2 Description of Experimental Results . . . . . . . . . . . . . . . 44

3.2.1 Arc behavior with no external magnetic field . . . . . . . . . 44

3.2 .2 Observed response of the $\operatorname{arc} \ldots \ldots \ldots \ldots \ldots$

3.3 Theoretical Model . . . . . . . . . . . . . . . . . . . . . . . . 47

3.3 .1 Assumptions . . . . . . . . . . . . . . . . . . 48

3.3.2 Derivation of Equation of Arc Shape . . . . . . . . . . . 49

3.3 .3 Arc length variation $\ldots \ldots \ldots \ldots$. . . . . . . . . 51

3.3.4 Effect of cathode spot motion . . . . . . . . . . . . 53

3.3 .5 Aerodynamic Drag . . . . . . . . . . . . . . . . . . 54

3.3.6 Effect of self magnetic field . . . . . . . . . . . . . 55

3.3.7 Non-uniform applied magnetic Field . . . . . . . . . . . 58

3.3.8 Range of validity of the model . . . . . . . . . . . . . 60

3.4 Application of Model to Experiment _. . . . . . . . . . . . . 62 
3.4 .1 Low Frequency Case . . . . . . . . . . . . . . . . . . 62

3.4 .2 Higher Frequencies . . . . . . . . . . . . . . . . . 63

3.4.3 Arc voltage and current variation . . . . . . . . . . . 71

3.4 .4 Self-field effects . . . . . . . . . . . . . . . . 73

3.5 An Industrial Application _ . . . . . . . . . . . . . . . 74

3.6 Discussion and Conclusions . . . . . . . . . . . . . 75

$\begin{array}{lll}4 & \text { Arc Instability } & 77\end{array}$

4.1 Experimental Setup . . . . . . . . . . . . . . . . 77

4.2 Experimental Results . . . . . . . . . . . . . . . . . . . 78

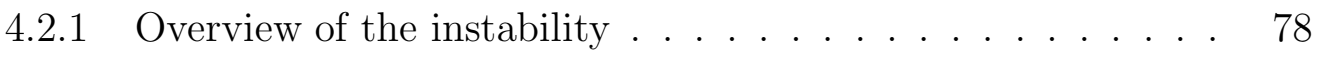

4.2.2 Cathode geometry dependence . . . . . . . . . . . 85

4.2 .3 Cathode spot motion . . . . . . . . . . . . . . . . 89

4.2.4 Erosion rate of the cathode . . . . . . . . . . . 92

4.3 Model of the Instability . . . . . . . . . . . . . . . . . . 100

4.3.1 Mechanism of the instability . . . . . . . . . . . . 100

4.3.2 Derivation of arc shape based on spot motion model . . . . . 102

4.3.3 Arc length variation due to spot motion . . . . . . . . 105

4.3.4 Application of the Spot Motion Model to the Instability Experiments . . . . . . . . . . . . . 107

4.4 Cathode processes affecting arc stability . . . . . . . . . . 112

4.4.1 Cathode temperature distribution influence on spot mobility 114

4.4 .2 Possible reasons for spot motion . . . . . . . . . . . . . . . 119

4.5 Industrial Application _ . . . . . . . . . . . . . . . . 128

4.6 Discussion and Conclusions _ . . . . . . . . . . . . . . . 129

4.6.1 The cathode spot motion model and its relevancy to experimental observations . . . . . . . . . . . . . . . . . . 129

4.6.2 Mechanism of the cathode spot motion . . . . . . . . . 132

4.6.3 Other possible models of the instability . . . . . . . . . . 132 
5 Conclusions and Future Work

5.1 Arc Deflection . . . . . . . . . . . . . . . 135

5.1.1 Conclusions ...................... 135

5.1.2 Suggestions for future work . . . . . . . . . . . 136

5.2 Arc Instability . . . . . . . . . . . . . . . . . . . . 137

5.2.1 Conclusions ..................... 137

5.2.2 Suggestions for future work . . . . . . . . . . . . 138

A Force due to Self-Magnetic Field of the Arc Column 143

B Exploratory Study of Arc Instability in Applied Axial Magnetic $\begin{array}{ll}\text { Field } & 147\end{array}$

B.1 Axial Field Experimental Setup . . . . . . . . . . . . . . 147

B.2 Experimental Results . . . . . . . . . . . . . . . . . . . 148

B.3 Speculation on the Mechanism of Instability in Axial Field . . . . . 156

$\begin{array}{lr}\text { Bibliography } & 159\end{array}$ 


\section{Chapter 1}

\section{Introduction}

\subsection{Uses of Atmospheric Pressure Arcs}

Thermal plasma generators in the form of electric arcs are used extensively in materials processing [1], metallurgy [2] and welding [3]. The work in this thesis was motivated particularly by the steelmaking application of the electric arcs. Electric arc furnaces are used primarily for remelting and recycling used steel. In 1998, electric arc furnaces were used to produce approximately $34 \%$ of the world's total steel output [4], with their use projected to grow. Improvements to the operation of these devices could thus have a large economic impact.

A schematic of a DC electric arc furnace as used in the steelmaking industry is shown in Figure 1.1. The arc in the furnace is used as a resistive heating element: most of the electrical power is dissipated in the arc because its resistivity is much higher than that of the rest of the circuit elements (the resistivity of the arc plasma at $\approx 15,000 \mathrm{~K}$ is $\sim 1000$ times higher than that of steel). Moreover, much of the power developed in the arc is carried directly to the anode (the steel to be melted) convectively via the strong magnetically pumped jet originating at the cathode (see Section 1.3.2). To further increase the melt rate and efficiency, and to decrease the wear of the furnace lining, the arc is usually kept submerged in a foamy slag. 


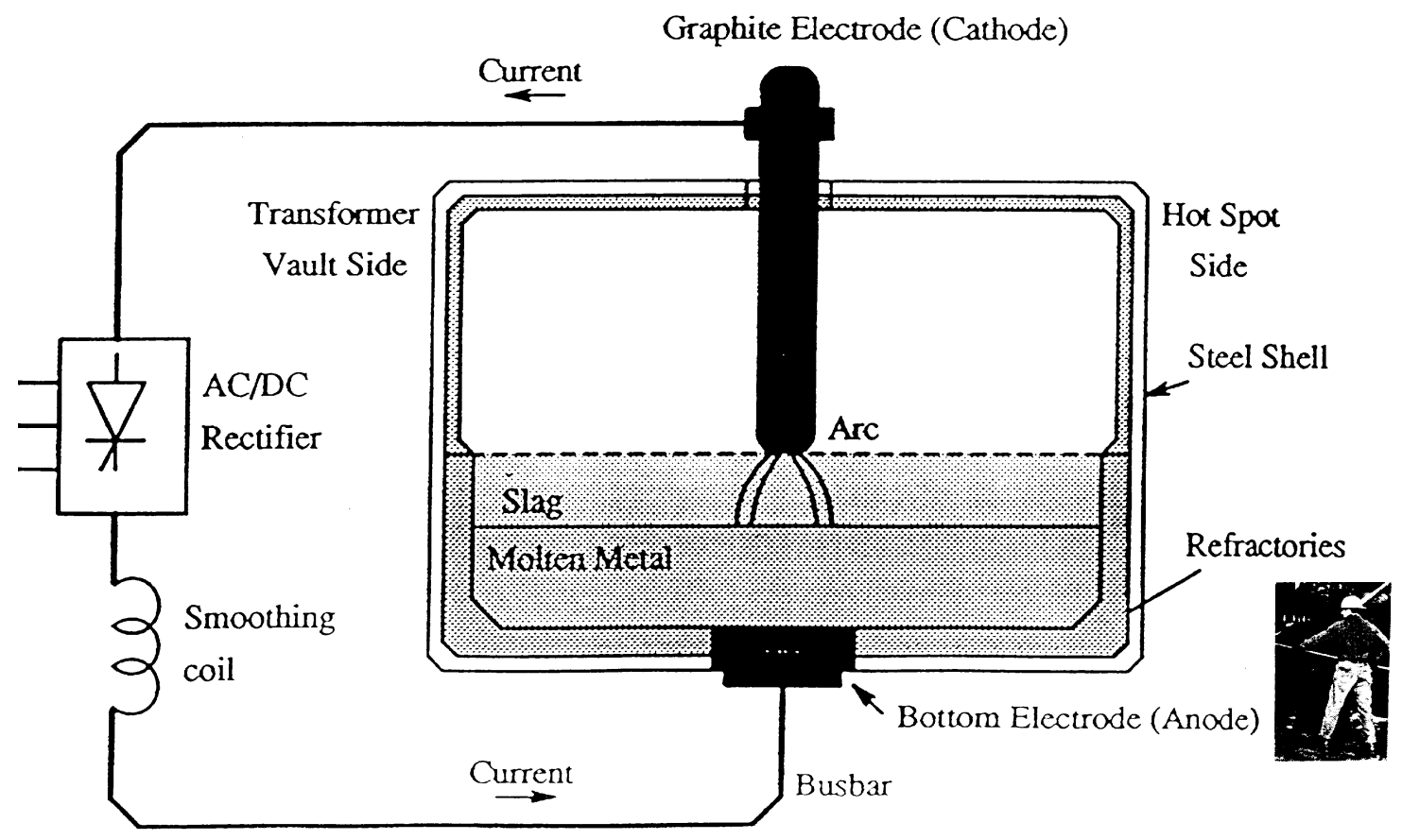

Figure 1.1: A schematic showing the main components of a DC electric arc furnace used in steelmaking. A worker is pictured on the lower-right for scale. Figure after Ref. 7.

Two issues of importance to the industry are arc deflection and arc instabilities. The former apparently occurs due to the stray magnetic fields from the electrical power cables feeding the arc furnace, shown schematically in Figure 1.1. The steady-state, uncontrolled, and often unmeasured deflection of the arc to one side causes increased wear of the refractory lining of the furnace, both via an increased heat flux and molten metal splatter [5-7]. The time-dependent arc instabilities cause "flicker" — voltage fluctuations on the electrical power grid to which the arc furnace is connected, which, without sufficient external electrical filtering, can cause noticeable variation in brightness of electric lights in the vicinity [7].

An additional detriment of the arc deflection and instabilities is a limitation on how long an arc can be sustained in the furnace. During the furnace operation, it is desirable to make the arc as long as possible in order to keep the voltage high and couple the most power into the furnace. Operating at a higher voltage rather than current keeps the current-dependent graphite electrode wear to a minimum, 
and lowers the capital cost of the rectifier circuitry. However, the amount of arc deflection, the voltage fluctuations, and the risk of arc extinction due to instabilities increase with the arc length, putting a limitation on how long an arc can be made.

Steelmaking electric arc furnaces are difficult to study and diagnose because of the very harsh environment of the furnace. A typical modern DC furnace releases approximately $100 \mathrm{MW}$ continuous power inside the furnace shell with on the order of $100 \mathrm{kA}$ of arc current [2]. Because of the presence of molten metal and vapors, and lack of diagnostic viewports, even photographing the arc is a challenge [2]. Moreover, the economics of the steelmaking business often preclude dedication of operating time to experimental studies. In part due to these reasons, and in part due to the complexities of arc physics associated with the interplay of aerodynamic, electromagnetic, plasma, and plasma-surface interaction effects, the physics of higher current arcs are not well understood despite their wide applications [2].

\subsection{Objectives of this Thesis}

The aims of this work are twofold. Firstly, it is to understand the arc motion due to external magnetic fields transverse to the arc current. This understanding is potentially applicable to the control of deflection in industrial arc furnaces. Secondly, it is to identify the causes of arc instabilities, which might lead to a better control of the instabilities in the industrial arcs.

The research was thus carried out with the following specific objectives in mind:

- Construct an experimental facility that allows the study of arc dynamics and that is flexible in configuration, has good diagnostic access, and, at the same time, retains similarity to the industrial installations, albeit on a much smaller scale.

- Understand the arc behavior in applied magnetic fields.

- Identify the cause of arc instabilities present in this device. 
The work corresponding to the objectives listed above is described in Chapters 2,3 , and 4 , respectively.

\subsection{An Overview of the Physics of Atmospheric Pressure Arcs}

Electric arcs were one of the first plasmas produced in a laboratory. Despite their importance in industry, knowledge of arc physics is far from complete. The type of electric arc under consideration belongs to the class of high-pressure, high intensity arcs, which burn at atmospheric pressure, with carbon cathode and a molten metal anode. The literature on arc equilibrium is very extensive, and only a brief overview will be given below. Some general references on the subject include Ref. 8-12.

\subsubsection{Arc Structure}

A schematic of a free-burning atmospheric arc with a carbon cathode is shown in Figure 1.2.

The description of atmospheric arc structure is usually divided into three regions: the cathode region, the arc column, and the anode region [13]. Though there exists extensive literature on mechanisms of cathode emission in arcs, many cathodic phenomena, such as cathode spot motion and the extreme current density on non-refractory metals, remain without a satisfactory explanation [13]. Cathodes made from refractory materials such as carbon or tungsten, however, are well described by thermionic emission. The cathode in a self-sustaining carbon arc operates at temperatures close to $3,500 \mathrm{~K}$ and higher, allowing electrons to boil off the surface. Electrons thus released are accelerated through a thin collisionless layer of net positive space charge with a relatively high electric field $\left(\sim 10^{5} \mathrm{~V} / \mathrm{cm}\right)$ into a collisional quasineutral layer where most of ionizations take place via electron impact. Most of the ions that heat the cathode are produced here. The ions move up the cathode sheath, impact the cathode and become neutralized, giving up some of 


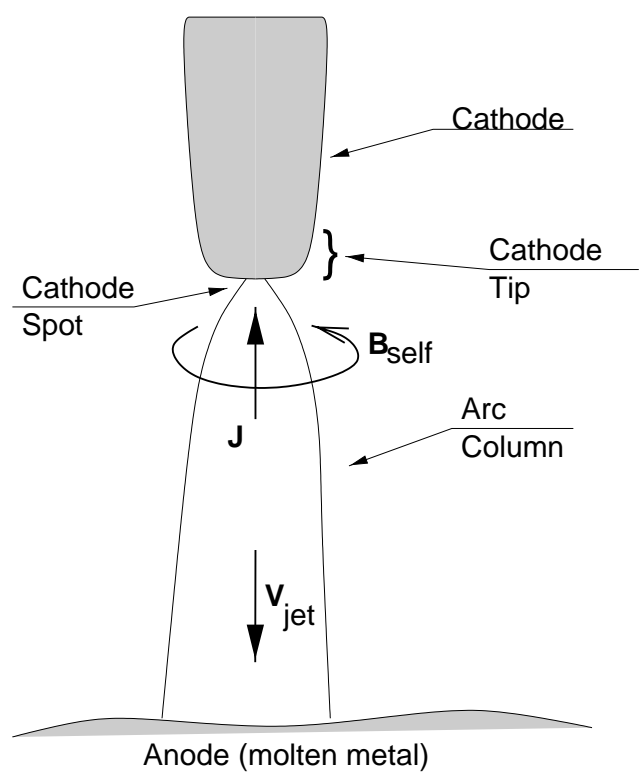

Figure 1.2: A schematic diagram of an electric arc.

their kinetic and recombination energy to the cathode, thus maintaining the cathode's high temperature [9]. The arc is constricted at its cathode attachment region, which is limited to a hot thermionically emitting spot on the surface (cathode spot), with current density of order $4 \times 10^{3} \mathrm{~A} / \mathrm{cm}^{2}[9]$.

The column of a high-pressure arc is usually taken to be in local thermodynamic equilibrium (LTE). Although all of the conditions for LTE are rarely satisfied, results of analysis done under LTE assumptions are reasonable approximations in most cases [13]. High collisionality and low electric field in the column at high pressure and high current keeps the difference in electron, ion, and neutral gas temperatures negligible. The column contains a narrow core region at temperatures of order $1 \mathrm{eV}$ and electron density of $10^{16}-10^{17} \mathrm{~cm}^{-3}$. The electron density falls off sharply with radius due to exponential dependence of ionization fraction on temperature.

Orders of magnitude of arc parameters (taken from the literature) for the present experiments and for a modern industrial arc furnace are listed in Table 1.1. It was not possible to reproduce the measurements of density, temperature, etc. in the experiments due to time limitations. 


\begin{tabular}{|c|c|c|c|}
\hline \multirow[b]{2}{*}{ Parameter } & \multirow[b]{2}{*}{ Definition } & \multicolumn{2}{|c|}{ Magnitude } \\
\hline & & PPPL Arc & Industrial Arc \\
\hline Power (peak) & $V I$ & $25 \mathrm{~kW}$ & $100 \mathrm{MW}$ \\
\hline Temperature & $T_{e}=T_{i}=T_{n}$ & $1 \mathrm{eV}$ & $1-2 \mathrm{eV}$ \\
\hline E-field (arc column) & $E$ & $10 \mathrm{~V} / \mathrm{cm}$ & $10 \mathrm{~V} / \mathrm{cm}$ \\
\hline Current (peak) & $I$ & $250 \mathrm{~A}$ & $100 \mathrm{kA}$ \\
\hline Self B-field & $B_{\text {self }}$ & $200 \mathrm{G}$ & $6 \mathrm{kG}$ \\
\hline Pressure & $p=n k T$ & $1 \mathrm{~atm}$ & $\gtrsim 1 \mathrm{~atm}$ \\
\hline$e^{-}$Density (peak) & $n_{e}$ & $10^{17} \mathrm{~cm}^{-3}$ & $\gtrsim 10^{17} \mathrm{~cm}^{-3}$ \\
\hline Characteristic Arc Dia. & $L$ & $0.6 \mathrm{~cm}$ & $10 \mathrm{~cm}$ \\
\hline Cathode Spot Radius & $r_{c}$ & $1.3 \mathrm{~mm}$ & $2.5 \mathrm{~cm}$ \\
\hline Ionization fraction (\%) & $n_{e} / n$ & $\sim 10 \%$ & $10-100 \%$ \\
\hline Debye Length & $\lambda_{D}=\sqrt{\frac{k T \epsilon_{0}}{4 \pi n_{e}^{2}}}$ & $10^{-6} \mathrm{~cm}$ & $10^{-6}$ \\
\hline Plasma Parameter & $(4 \pi / 3) n_{e} \lambda_{D}^{3}$ & $\sim 5$ & $\sim 5$ \\
\hline Plasma Frequency & $f_{p e}=\sqrt{\frac{n_{e} e^{2}}{4 \pi^{2} \epsilon_{0} m_{e}}}$ & $10^{12} \mathrm{~Hz}$ & $10^{12} \mathrm{~Hz}$ \\
\hline Ion Gyrofrequency & $f_{c i}=\frac{e B_{\text {self }}}{2 \pi m_{i}}$ & $10^{4} \mathrm{~Hz}$ & $10^{5} \mathrm{~Hz}$ \\
\hline Ion Gyroradius & $\rho_{L i}=\frac{\sqrt{k T / m_{i}}}{2 \pi f_{c} i}$ & $2 \mathrm{~cm}$ & $0.6 \mathrm{~mm}$ \\
\hline$e^{-}$Collision Frequency & $\nu_{e}=\frac{n_{i} e^{4} \ln \Lambda / \pi^{2} \epsilon_{0}^{2}}{12 \sqrt{2 m_{e}}\left(k T_{e}\right)^{3 / 2}}$ & $10^{11} \mathrm{sec}^{-1}$ & $10^{11} \mathrm{sec}^{-1}$ \\
\hline$e^{-}$Mean Free Path & $\lambda_{\mathrm{mfp}, e}=\frac{\sqrt{k T / m_{e}}}{\nu_{e}}$ & $10^{-5} \mathrm{~cm}$ & $10^{-5} \mathrm{~cm}$ \\
\hline Conductivity & $\sigma=\left(I / \pi L^{2}\right) / E$ & $80 \Omega^{-1} \mathrm{~cm}^{-1}$ & $100 \Omega^{-1} \mathrm{~cm}^{-1}$ \\
\hline Resistive Diffusion Time & $\tau_{R}=\mu_{0} L^{2} \sigma$ & $10^{-7} \mathrm{sec}$ & $10^{-4} \mathrm{sec}$ \\
\hline Alfvén Speed & $V_{A}=B_{\text {self }} / \sqrt{\mu_{0} \rho}$ & $10^{4} \mathrm{~cm} / \mathrm{s}$ & $10^{5} \mathrm{~cm} / \mathrm{s}$ \\
\hline Magnetic Reynolds \# & $R_{m}=\tau_{R} V_{A} / L$ & $10^{-3}$ & $\sim 1$ \\
\hline Plasma $\beta$ & $\beta=\frac{p}{\left(B_{\text {self }}^{2} / 2 \pi \mu_{0}\right)}$ & $\sim 1000$ & $\sim 1$ \\
\hline Plasma jet speed (peak) & $v_{\mathrm{jet}}=I \sqrt{\frac{\mu_{0}}{2 \pi^{2} r_{c}^{2} \rho}}$ & $10^{4} \mathrm{~cm} / \mathrm{s}$ & $10^{5} \mathrm{~cm} / \mathrm{s}$ \\
\hline Mach number (peak) & $M$ & $10^{-2}$ & $\sim 1$ \\
\hline Hydrodyn. Reynolds \# & $R_{H}=v_{\mathrm{jet}} r_{c} / \eta$ & 10 & $10^{3}$ \\
\hline
\end{tabular}

Table 1.1: Orders of magnitude of arc parameters in the present experiments and in a modern industrial arc furnace. 


\subsubsection{Physics of the cathode jet}

An important feature of high-intensity arcs is a jet originating due to the current constriction at the cathode $[8,14]$. The mechanism of the jet formation is as follows.

Due to the constriction at the cathode needed to maintain electron emission, the self-magnetic field of the arc is highest there and decreases with axial distance away from the cathode (see Figure 1.2). Because of the high collisionality, pressure is isotropized, and the axial gradient in the self-magnetic field induces an axial pressure gradient. This pressure gradient drives an axial flow directed away from the cathode, while colder surrounding gas is entrained in the constriction region.

The maximum velocity of this jet has been estimated by Maecker [15] assuming no viscosity and a uniform current density. Following the derivation in Ref. 8, assume that all of the $\vec{J} \times \vec{B}$ force is balanced by the radial pressure gradient:

$$
\frac{d p}{d r}=-J(r) B(r)
$$

The overpressure $q \equiv p-p_{\text {atmospheric }}$ on the axis of the discharge $(r=0)$ is then given by:

$$
q=\int_{R}^{0} \frac{d p}{d r} d r=\int_{0}^{R} J(r) B(r) d r
$$

where $R$ is the radius of the conducting arc boundary. Assuming uniform current density, $J(r)=J$, the self magnetic field is given by:

$$
B(r)=\frac{\mu_{0}}{2 \pi r}(J)\left(\pi r^{2}\right)=\frac{\mu_{0} J r}{2}
$$

Substituting Eq. (1.3) into Eq. (1.2), get:

$$
q=\frac{\mu_{0} J^{2} R^{2}}{4}=\frac{\mu_{0} I^{2}}{4 \pi^{2} R^{2}}
$$


where $I$ is the arc current. The pressure difference between to axial locations in the arc, with conducting radii $R_{1}$ and $R_{2}$, then is:

$$
\Delta q=\frac{\mu_{0} I^{2}}{4 \pi^{2}}\left(\frac{1}{R_{1}^{2}}-\frac{1}{R_{2}^{2}}\right)
$$

Taking $R_{1}=r_{c}$, where $r_{c}$ is the cathode spot radius, letting $R_{2}$ be the column radius, and assuming $r_{c}^{2} \ll R_{2}^{2}$, the maximum axial pressure difference is

$$
\Delta q=\frac{\mu_{0} I^{2}}{4 \pi^{2} r_{c}^{2}}
$$

The axial momentum balance is given by:

$$
\frac{d q}{d z}=\rho v \frac{d}{d z} v=\frac{\rho}{2} \frac{d\left(v^{2}\right)}{d z}
$$

where $\rho$ is an average mass density on axis. Integrating Eq. (1.7) from the cathode $(z=0)$ to a location in the column, get:

$$
\Delta q=\frac{\rho v^{2}}{2}
$$

Substituting Eq. (1.6) into the above and solving for $v$, finally obtain the result found by Maecker [15]:

$$
v_{\text {peak }}=I \sqrt{\frac{\mu_{0}}{2 \pi^{2} r_{c}^{2} \rho}}
$$

where subscript "peak" intends to emphasize that this is an upper estimate of the peak velocity on axis, before viscous effects broaden the velocity profile.

As will be seen in Chapters 3 and 4, the jet plays an important role in determining the arc dynamics. 


\subsection{An Overview of Previous Work}

\subsubsection{Arc deflection}

The interaction of various arcs with applied DC magnetic fields has been studied extensively with regard to applications such as magnetically rotated arcs, driven arcs, and magnetically balanced arcs $[10,16]$. In these applications, the arc is moving as a whole with respect to the surrounding atmosphere. The applied magnetic field experiments described in Chapter 3 pertain to the magnetic deflection of a DC arc which is free to move on a large area anode and is fixed at an emission spot on the cathode. In this case, as will be seen below, the arc is deformed by the applied magnetic field. This type of setup is found in welding and arc furnace applications, where, as discussed above in Section 1.1, arc deflection due to stray magnetic fields from the electrical power feeds can cause uneven melting or furnace wall hot spots and erosion [5-7]. However, no consistent quantitative experimental analysis of a high intensity ( $\gtrsim 100 \mathrm{~A}$ ) arc motion in this geometry in response to magnetic fields from DC to high frequencies is found in the literature.

Maecker [17] has given a general description of arc motion and equilibrium in external magnetic and flow fields. That description, however, becomes inadequate for high intensity $\operatorname{arcs}(I \gtrsim 100 \mathrm{~A})$, where strong plasma flows generated by magnetic pumping effect $[8,14]$ at the cathode become important. In [18], experiments on a low current $(I<12 \mathrm{~A})$ arc subjected to an oscillating transverse magnetic field of up to $100 \mathrm{~Hz}$ are analyzed on the basis a balance of the Lorentz $\vec{J} \times \vec{B}$ force, inertia, and aerodynamic drag. Ref. 19 examined a low current arc in DC transverse magnetic field with fixed anode and cathode regions on the basis of inhomogeneous heat flow. Speckhofer et al. [20] performed experiments and three-dimensional numerical modeling on a short (3 mm) $100 \mathrm{~A}$ arc in transverse DC magnetic field for a conical tungsten cathode.

A qualitative description of the behavior of an arc similar to that studied in the present experiments was given in [21] for $\mathrm{DC}$ and $50 \mathrm{~Hz} \mathrm{AC}$ applied magnetic field, together with a discussion of the forces acting on the moving column of the arc. Lancaster [3] gives a description of magnetic field effects in welding, as well 
as empirical and analytic expressions for gas tungsten welding arc deflection in DC fields.

\subsubsection{Arc instability}

The literature contains several cases of direct comparisons between theories of arc instabilities and experiments. All of these cases, however, involve low intensity arcs, with currents of order $\sim 10 \mathrm{~A}$, where electrode jet effects are insignificant. Hülsmann and Mentel [22,23] have done a comprehensive study of stability of a low current $(\sim 10 \mathrm{~A})$, long, wall stabilized atmospheric pressure hydrogen arc. Gaede [24] investigated a free-burning $\sim 10$ A hydrogen arc with one end fixed by a diaphragm and the other free to move in a plane.

A helical instability of a wall-stabilized hydrogen arc burning in a $3 \mathrm{~mm}$ dia. tube at 500-900 torr pressures and currents up to 120 A was studied experimentally in Ref. 25. The onset of the instability was explained on the basis of the competition between the destabilizing self-magnetic forces and the stabilizing effect of the wall and inhomogeneous heat flow tending to straighten the current path (Section 3.3.8). The onset of instability was found to depend only on a parameter containing the input power and the average plasma enthalpy, mass density, viscosity and conductivity.

A recent theoretical work [26] examined arc stability in external axial magnetic field. Instability was studied for an infinitely long arc column on time scales slower than the resistive and viscous diffusion time scales. It was concluded that the arc is unstable to helical perturbations with wavelengths on the order of $2 \pi \times$ (arc radius) with the axial magnetic field, and marginally stable to such perturbations with no external magnetic field applied. The analysis was carried without any zeroorder flows, such as the cathode jet. The paper states that the case with flow will be the subject of a future publication.

Several investigations had focused on stability of high-current arcs in an imposed accelerating flow, such as found in gas-blast circuit breakers and arc heaters. In Ref. 27, a numerical study of a $500 \mathrm{~A} \mathrm{SF}_{6}$ arc at 8 atm in imposed accelerating flow finds instability due to shear flow amplification of density fluctuations. 
In Ref. 28, an experimental investigation of a 20-500 A AC arc in accelerating flow revealed similarities with the instabilities seen in fluid jets. An axisymmetric disturbance was generated, which developed into a helical mode downstream. The arc was stable for Reynolds numbers $<100$. The work concludes that the instabilities arose from the shear layers between the cold gas flow and the more accelerated plasma of the arc column. Ref. 29 describes experiments on a nitrogen arc in accelerating flow at 25 atm. The helical instability observed was analyzed on the basis of destabilizing self-magnetic forces and convective stabilization by the imposed flow. Qualitative agreement was found between the analysis and the experiments.

Ragaller [30] derived the functional form of linear stability behavior of an infinitely long arc column against the $\mathrm{m}=1$ kink mode with the aid of dimensional analysis. Stability was determined by the balance of the self-magnetic field of the helically disturbed column and asymmetrical heating effects. It was assumed that axial flow can be treated by choosing a reference frame moving with the flow, and it was are not included in the treatment. It is mentioned, however, that the flow can stabilize the arc if the instability growth rate is slow enough.

Bellan et al. [31] performed experiments on arc blowout initiated by transverse magnetic field on a pulsed, $\approx 1 \mathrm{kA}, 0.6 \mathrm{~cm}$ long arc, with the discharge lasting $\approx 40 \mathrm{~ms}$. The blowout was treated as being driven by the self-magnetic field of the arc and the centrifugal force of the jet flow. Stabilization of the arc blowout was accomplished with an external axial magnetic field on the order of the self-field of the arc. However, the axial field was also found to induce helical rotation of the arc column. It is suggested that the applied axial field was partially "frozen" into the plasma, because of the arc's high conductivity, and stabilized the blowout through the resistance of the field lines to bending (similar to Kruskal-Shafranov kink stability criterion for a z-pinch). Further, as the flux was only partially "frozen in" (the Magnetic Reynolds number was estimated to be $\sim 0.3$ ), it produced a torque on a helically perturbed arc column, resulting in column rotation.

$\mathrm{AC}$ arcs with a geometry similar to the present experiments (free-burning between a graphite rod and a steel plate), but at an order of magnitude higher currents $(\approx 10 \mathrm{kA} \mathrm{AC})$ were observed by Bowman and Jordan [32]. The arc was observed to be stable during the half-cycle with the graphite as cathode (the case 
in the present experiments), and contorted by "kinks" when the graphite was the anode. A strong cathode jet was present during the former, and absent in the latter. The stability in the former case was explained by the stabilizing effect of the cathode jet momentum, which precluded self-magnetic "kinking". Through a simplified analysis balancing the force per unit length due to self-magnetic field of a helical column with the centripetal force of the jet, it was found that the stabilizing effect of the cathode jet precludes instability for wavelengths shorter than $\approx R^{3} / r_{c}^{2}$, where $R$ is the column radius, and $r_{c}$ is the cathode spot radius. It was concluded that the arc was stable in this case because the minimum wavelength for instability was longer than the arc length. Additionally, the work discusses the limitation on the angle the arc column makes with the graphite electrode axis on the basis of interaction of the current distribution in the graphite cathode with the arc current.

The instability studied in the present work (Chapter 4) is first mentioned in Ref. 11 for free burning arcs between graphite electrodes for currents greater than 400 A. Witkowski [33] investigated this instability experimentally and suggested a fire-hose type instability mechanism. Montgomery and Sharp [34] observed the instability for an arc between graphite rod cathodes and a graphite plate anode in the range of 400-2000 A. It was found that the current threshold for the instability depended on the geometry of the cathode tip: the arc was stabilized by the presence of a sharp edge on the cathode, and became unstable as soon as the edge was eroded. The arc was stable with pointed cathodes, but became unstable as the point eroded. The frequency of column motion was found to be $\approx 1.5 \mathrm{kHz}$, and was independent of current in the range 400-2000 A, and weakly dependent on arc length and cathode diameter. It was suggested that this dependence is a result of the influence of the current distribution in the cathode on arc stability.

\subsubsection{Summary}

The following briefly summarizes the above overview of the previous work. 


\section{Arc deflection}

It has been established in previous investigations that arc displacement in applied magnetic fields at atmospheric pressure is due to the $\vec{J} \times \vec{B}$ force on the arc current. It has also been found that the response of the arc often depends on the specific geometries of the electrodes, the electrode materials, and the current range. However, no detailed measurements and analysis of arc deflection in the geometries relevant to arc furnaces is found in the literature.

\section{Arc Instability}

Detailed experimental and theoretical analysis of arc column instabilities is found in the literature for low current $(\leq 100 \mathrm{~A})$ arcs. For such arcs, the destabilizing mechanism is usually found to be a magnetic "kink". Experimental studies usually aim to minimize the influence of the electrodes on the discharge and theoretical analysis tends to disregard their effects.

Experiments and analysis is scarce for free-burning higher-current arcs. There are conflicting conclusions as to the stabilizing or destabilizing role of the cathode jet (e.g. Bowman [32] vs. Bellan [31]). Cathode geometry effect is found to be critical to stability [34]. However, no satisfactory explanation of the effect is found.

Experimental studies of industrial scale arcs $(\sim 100 \mathrm{kA})$ are based on limited visual observations of instabilities, with analysis usually highly speculative, consisting mostly of extrapolations from lower currents or from fluid mechanic phenomena. However, it is clear that such arcs are almost always highly unstable $[2,35,36]$.

Possible instability mechanisms in industrial scale $(\sim 100 \mathrm{kA})$ arc furnaces were characterized by Bowman [2] as follows:

- Aerodynamic: instabilities driven by the high-speed cathode jet. Further subdivided into helical ("firehose"-like) and axisymmetric vortex-like.

- Magnetic: driven by the self-magnetic forces ("kink" instability). 
- Cathode spot movement: disturbance of the arc column due to motion of the arc attachment spot on the cathode.

\subsection{Similarities and Differences of the PPPL Arc and the Industrial Furnace Arcs}

The arc furnace setup under study in this thesis is a very much scaled down version of the industrial steelmaking furnace. It is approximately four orders of magnitude smaller in power and three orders of magnitude lower in current. However, it retains many similar features important in determining the arc behavior. Among the structural similarities are: a free-burning arc, the same geometry and composition of electrodes (movable graphite cathode, large area steel anode), as well as the same working gas and pressure (air at 1 atm). Structural differences include the absence of foamy slag surrounding the arc and a steel shell (usually ferromagnetic) surrounding the furnace, which are found in industry.

Arc physics at the two scales retain many similarities as well. As can be seen in Table 1.1, plasma temperature, electric field, electron density, collisionality, mean free path, conductivity, lack of magnetization (collision frequency $\gg$ gyrofrequency) are all similar. Moreover, the cathode jet remains a dominant feature in both.

Several important differences can be pointed out. The higher self-magnetic field in the industrial case implies a higher Magnetic Reynolds number. Whereas in the case of the present experiments the magnetic field evolution is dominated by resistive diffusion $\left(R_{m} \sim 10^{-3}\right)$, the industrial arc is approaching the "frozen in" behavior of the magnetic field $\left(R_{m} \sim 1\right)$. Moreover, the ratio of plasma pressure to magnetic pressure (plasma $\beta$ ) is $\sim 1000$ for the experiments and $\sim 1$ for the industrial arc, indicating some degree of confinement. Hydrodynamic parameters differ as well: a factor of 100 in Reynolds number, and possibly supersonic cathode jet flow (Mach number $\sim 1$ ) in the case of the industrial arcs [2].

The last paragraph underscores the difficulties in analyzing arc phenomena on the scale found in industry. However, it is believed that successful measure- 
ments and analysis of the smaller scale phenomena will yield useful insight into this fascinating regime.

\subsection{Outline of the Thesis}

The thesis is organized as follows. The description of the experimental apparatus, the diagnostics, and the data acquisition system are given in Chapter 2 . Chapter 3 describes the experiments and results on the deflection of a stable arc in applied DC and AC transverse magnetic field. A simple analytical model for the arc dynamics in transverse field is developed and is used to analyze the DC and $\mathrm{AC}$ deflection in a consistent manner.

The discussion of unstable arc behavior is taken up in Chapter 4. Here, instability observations and the conditions under which instability occurs are discussed, and results of stability experiments are presented. A cathode spot motion model of the instability is developed on the basis of the model of arc dynamics from the transverse magnetic field study (Chapter 3), and is shown to be consistent with the experimental results. Possible reasons for the motion of the cathode spot on the surface of the cathode are discussed. The chapter is concluded with an application of the model to an industrial-scale arc furnace.

Conclusions and suggestions for future work are given in Chapter 5. Appendix A gives supplemental self-magnetic field calculations. Finally, Appendix B summarizes exploratory experiments on arc stability in an external axial magnetic field. 


\section{Chapter 2}

\section{Experimental Apparatus}

This chapter gives a description of the apparatus used in the arc deflection and instability experiments described in Chapters 3 and 4 . The chapter is organized as follows. Section 2.1 describes the hardware setup of the arc furnace, Section 2.2 describes the diagnostics used in the experiments, Section 2.3 gives the methods of data acquisition, and finally, arc $(I, V)$ characteristics are discussed in Section 2.4.

\subsection{Arc Furnace Hardware}

A side view of the experimental setup is shown schematically in Figure 2.1, and a top-down view of the setup geometry is shown in Figure 2.2. The arc is struck by contact between a vertical graphite rod cathode and a flat metal surface anode whose diameter is much larger than the arc diameter. The arc length is adjusted by moving the cathode using a remotely controlled stepper motor (Section 2.1.1). The arc is burning at atmospheric pressure in air. Within a minute after the arc is initiated by electrode contact, the metal surface of the anode becomes molten. A photograph of the inside of the arc furnace is shown in Figure 2.3. Photographs of several arcs in operation are shown in Figure 2.4.

Several different anode configurations where used: a $4^{\prime \prime}$ dia. iron cylinder enclosed in a $\mathrm{MgO}$ brick crucible impregnated with a conducting tar (such as used 


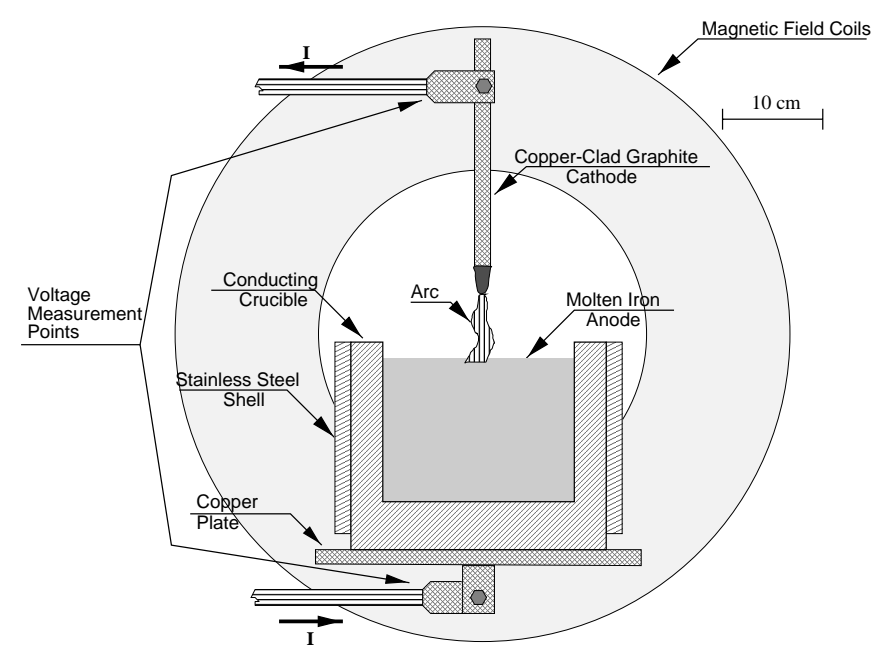

Figure 2.1: Experimental setup (side view). Shown is the iron anode in the $\mathrm{MgO}$ conducting crucible.

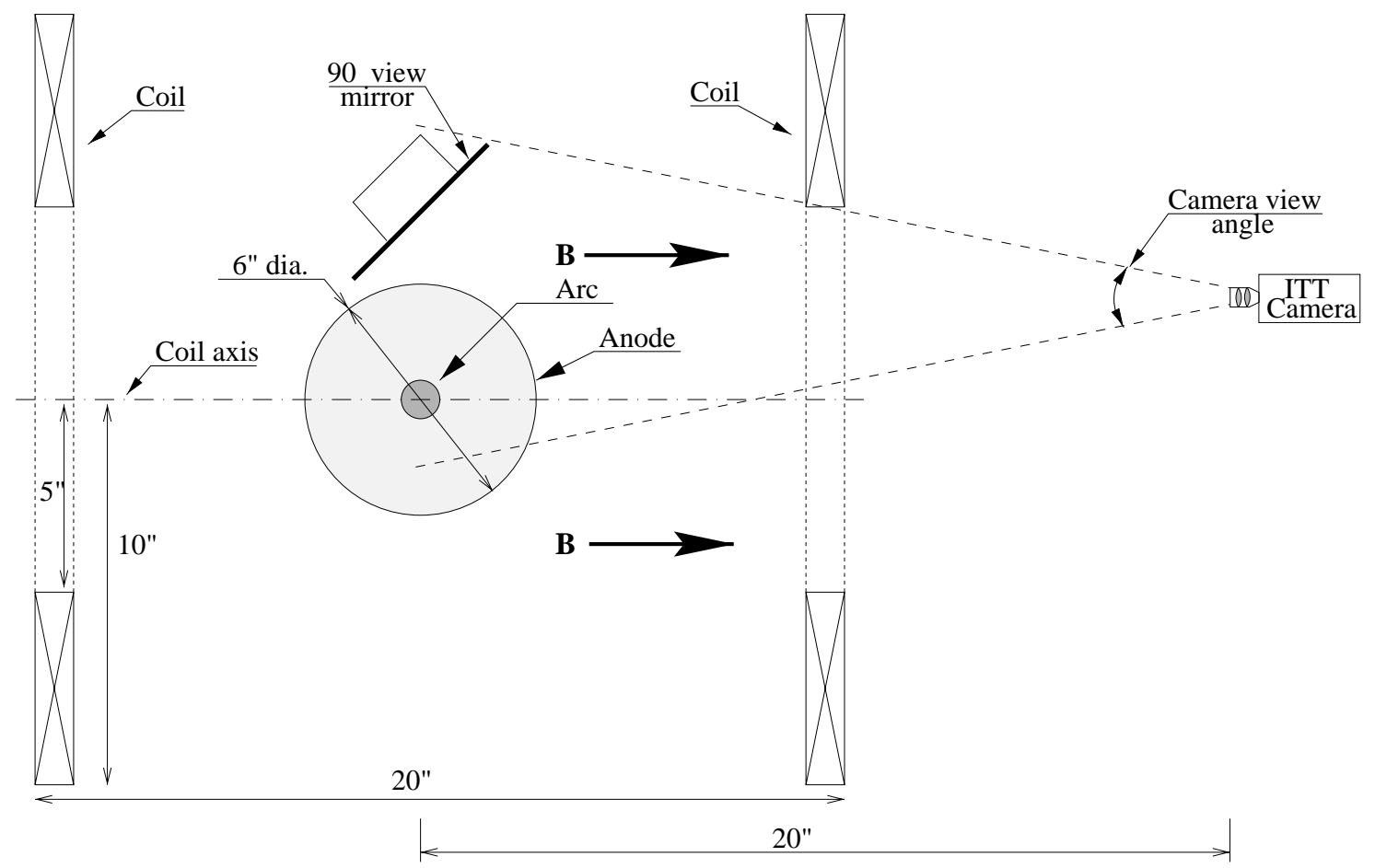

Figure 2.2: Experimental setup (top down view showing the geometry of the coils for the transverse magnetic field). Also shown are the ITT camera and the $90^{\circ}$ view mirror, referred to in Section 2.2.4. 


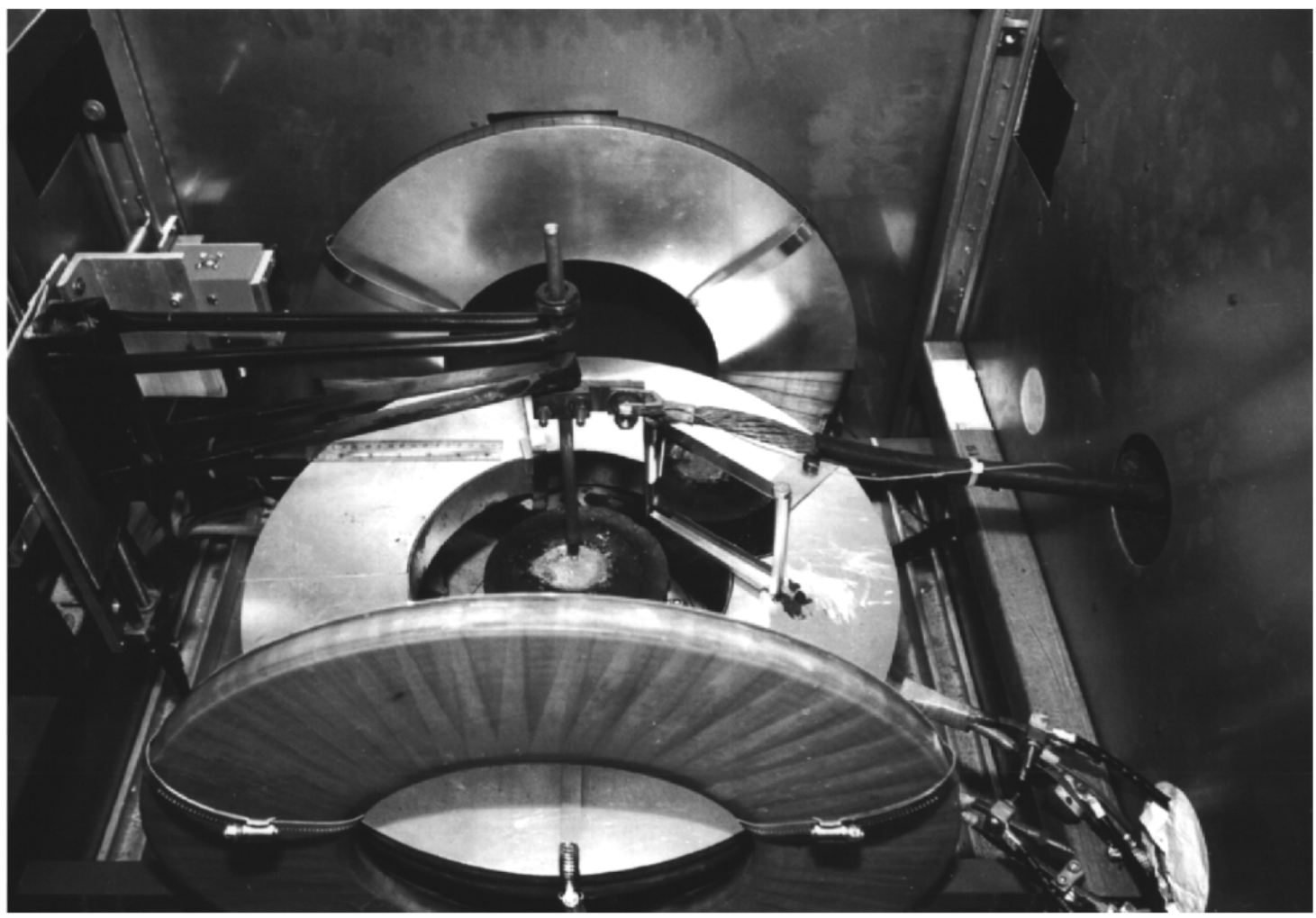

Figure 2.3: Inside of the arc furnace viewed from just above the ITT camera. The transverse magnetic field coils (20" outside diameter) are in the front and rear of the photo. A standard $3 / 8^{\prime \prime}$ cathode with the negative power supply lead attached is seen in the middle, with the anode below partially visible. The $90^{\circ}$ view mirror is to the right of the cathode.

in steelmaking arc furnaces), a $4^{\prime \prime}$ dia. non-magnetic stainless steel cylinder in a similar crucible, as well as a $4^{\prime \prime}$ and a $6^{\prime \prime}$ dia. non-magnetic stainless steel cylinders without crucibles. The iron anode configuration is shown as part of Figure 2.1, and a photograph of the $4^{\prime \prime}$ stainless steel anode inside the crucible is given in Figure 2.5. There was no perceptible difference in the arc behavior between these types of anodes.

The depth of the molten layer on the iron anode measured immediately after the shut-off of a $150 \mathrm{~A}$ arc was approximately $0.5 \mathrm{~cm}$. The temperature on the side surface of the anode was measured using a thermocouple to be approximately $650^{\circ} \mathrm{C}$ after $\approx 15$ min of running. 


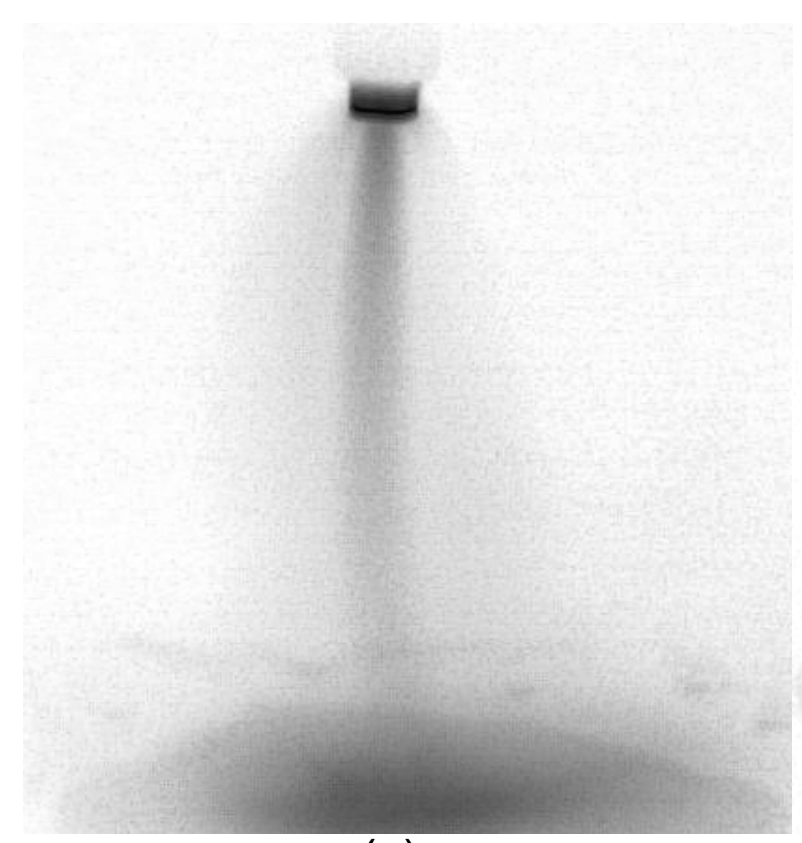

(a)

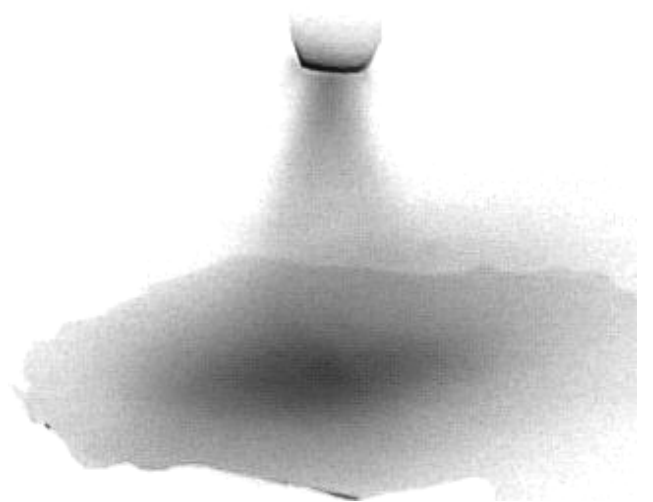

(c)

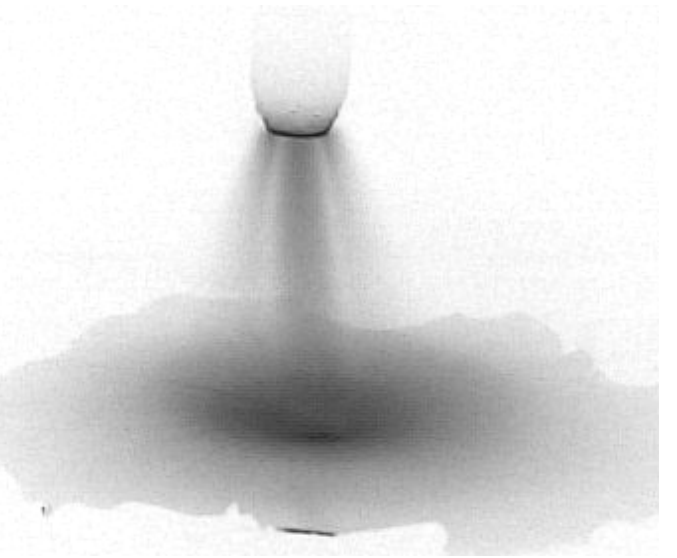

(b)

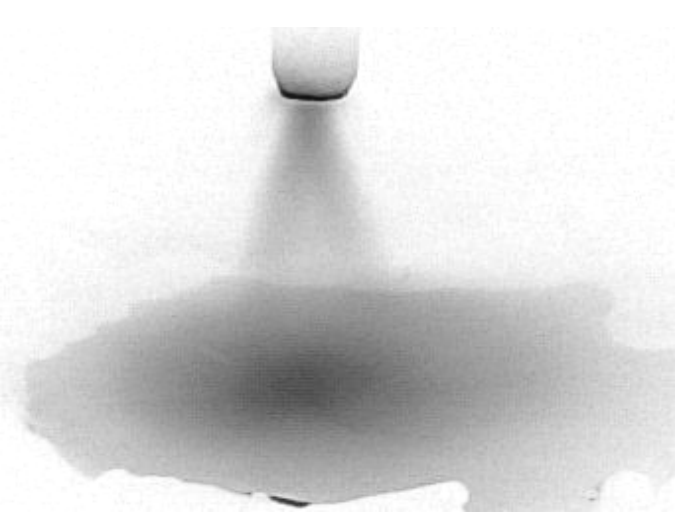

(d)

Figure 2.4: Negatives of stable ((a) and (b)) and unstable ((c) and (d)) arcs. Note the bright narrow core visible for the stable arcs and the cone shape of the unstable arcs (the exposure time is many instability periods).

The arc is powered using up to two current-regulated EMHP 80-250 power supplies, each rated at $80 \mathrm{~V}, 250 \mathrm{~A}$, with a maximum available power of $40 \mathrm{~kW}$. Both a single supply and two supplies in series have been used. A $0.12 \Omega$ ballast resistor is inserted in series with the arc during many of the runs. Two types of ballast resistors are used: an inductive one (helically wound, $L=30 \mu \mathrm{H}$ ) and a 


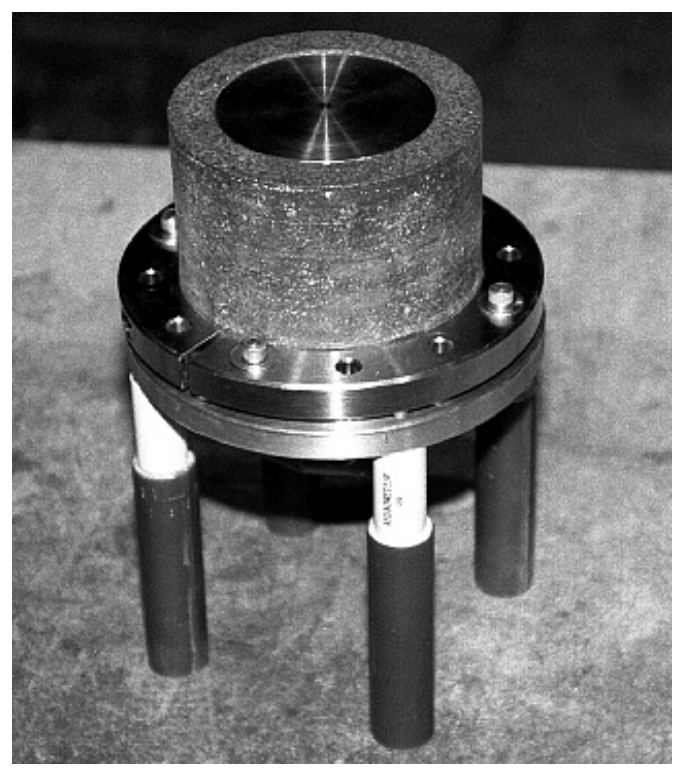

Figure 2.5: The $4^{\prime \prime}$ stainless steel anode inside the tar impregnated $\mathrm{MgO}$ crucible mounted on the stand prior to installation into the arc furnace.

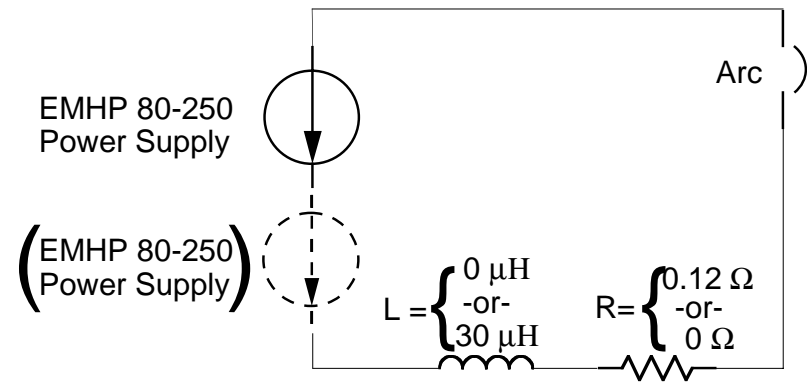

Figure 2.6: Schematic of the arc electrical circuit. Several configurations where used: one or two EMHP 80-250 current-regulated supplies with a non-inductive $(L=0)$ or inductive $(L=30 \mu \mathrm{H}) 0.12 \Omega$ ballast resistor, or no ballast resistor.

non-inductive one (steel ribbon, model Avtron AGR200LS). The arc deflection and arc instability were the same in all configurations: one or two power supplies, and either or none of the ballast resistors. A diagram of the arc circuit is shown in Figure 2.6. Typical operation is with two supplies in series and the non-inductive $(L=0) 0.12 \Omega$ ballast resistor.

Most of the voltage drop in the arc circuit occurs across the arc itself. Voltage of $\approx 18 \mathrm{~V}$ at $150 \mathrm{~A}$ was measured with the cathode in direct contact with the anode 
surface with the anode cold. Most of this voltage drop, however, occurs across a resistive layer of porous crust that forms on the anode surface as the metal solidifies. Once the arc has been initiated, this layer melts rapidly.

\subsubsection{Cathode position control}

The cathode holder consists of a chuck into which the standard copper clad graphite $3 / 8^{\prime \prime}$ welding rod is placed. Larger diameter cathodes are drilled, tapped, and threaded onto a $3 / 8^{\prime \prime}$ dia. copper rod, which is then inserted into the chuck. The negative lead from the power supply is clamped directly onto the standard copper clad graphite electrode or the $3 / 8^{\prime \prime}$ copper rod. The chuck is attached via a steel arm to a vertically movable stage. The stage is raised or lowered using a stepper motor linked to the stage using an electrically controlled clutch. The clutch is set for light slipping torque when lowering the cathode stage in order to slip when the tip of the cathode electrode comes in contact with the anode, and for heavy slipping torque when the stage is being raised. The range of motion of the stage holding the cathode is limited to $\approx 7 \mathrm{~cm}$, which determined the maximum arc length in these experiments.

\subsubsection{Transverse magnetic field experiments}

In the applied transverse magnetic field experiments, the set of coils pictured in Figure 2.1 is used to apply a magnetic field to the arc in the direction perpendicular to the arc current. A signal generator and an audio amplifier are used to drive the coils at single frequencies in the range $\mathrm{DC}-2 \mathrm{kHz}$. The coil driving circuit is represented in Figure 2.7, together with the equivalent resistance and inductance of the two coils. For the instability experiments, the coils were not energized.

The coils utilized are a pair of "L-2" pancake coils [37], with 33 turns each, $10^{\prime \prime}$ inner diameter and $20^{\prime \prime}$ outer diameter and 3/4" thickness. Each of the coils

produces 1.1 Gauss/A at its center on axis. In order to protect the epoxy-encased coils from the arc radiation, aluminum heat shields where attached to the coil sides 


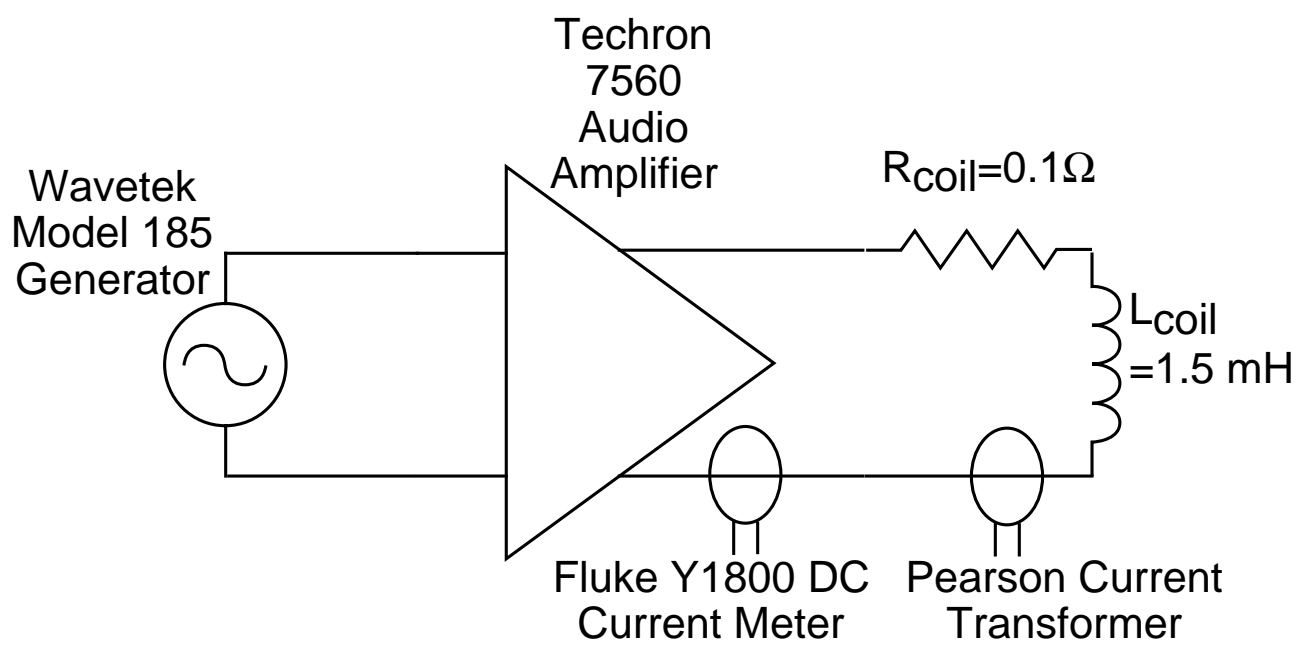

Figure 2.7: Schematic of circuit for driving the transverse magnetic field coils.

facing the arc on the upper halves of the coils. The shields are $1 / 32^{\prime \prime}$ thick. High pressure air was sent through the coils for additional cooling.

With the anode and crucible removed, the magnetic field at the arc (midway between the two coils) was measured to be $5.0 \pm 0.2$ Gauss, uniform to that tolerance along the $7 \mathrm{~cm}$ maximal arc length with $10 \mathrm{~A} \mathrm{DC}$ in coils. With a non-magnetic steel anode in place, the magnetic field measured was $5.3 \pm 0.2$ Gauss from the surface of the anode to $6 \mathrm{~cm}$ above it. The measurements were made using a Walker Scientific MG-50 gaussmeter with a 145R408 probe.

The magnetic field was also measured for the iron anode in crucible and non-magnetic steel anode for several frequencies of the applied magnetic field for several distances above the anode surface using a calibrated B-dot coil. The results of the measurements are shown in Tables 2.1 and 2.2.

The applied magnetic field strength available in the experiments was limited by the voltage output of the amplifier at higher frequencies to approximately 08 Gauss.

The results of these magnetic field measurements were used to analyze the applied transverse magnetic field experiments, described in Chapter 3. The amplitude of the applied magnetic field is a parameter in the model for the deflected arc shape. Since various frequencies in the range $\mathrm{DC}-1.6 \mathrm{kHz}$ were used in the ex- 


\begin{tabular}{|r||c|c|c|}
\cline { 2 - 3 } \multicolumn{1}{c|}{} & \multicolumn{3}{c|}{ Distance above the iron anode: } \\
\hline Frequency & \multicolumn{3}{c|}{ Magnetic Field (Gauss) } \\
\hline $40 \mathrm{~Hz}$ & 3.5 & 3.9 & 4.4 \\
$100 \mathrm{~Hz}$ & 3.9 & 4.6 & 4.6 \\
$400 \mathrm{~Hz}$ & 3.9 & 4.4 & 4.6 \\
$700 \mathrm{~Hz}$ & 3.9 & 4.3 & 4.4 \\
$1200 \mathrm{~Hz}$ & 3.8 & 4.1 & 4.2 \\
\hline
\end{tabular}

Table 2.1: Applied magnetic field variation with distance above the anode surface and applied field frequency for the iron anode. The amplitude of the current in the coils was fixed at 10.0 A peak.

\begin{tabular}{|r||c|c|c|c|}
\cline { 2 - 5 } \multicolumn{1}{c|}{} & \multicolumn{4}{c|}{ Distance above the steel anode: } \\
\hline Frequency & $1.0 \mathrm{~cm}$ & \multicolumn{4}{c|}{ Magnetic Field (Gauss) } \\
\hline $100 \mathrm{~Hz}$ & 4.9 & 5.1 & 4.9 & 4.9 \\
$700 \mathrm{~Hz}$ & 4.4 & 4.8 & 4.9 & 4.8 \\
$1000 \mathrm{~Hz}$ & 4.3 & 4.6 & 4.8 & 4.6 \\
$1500 \mathrm{~Hz}$ & 4.1 & 4.4 & 4.6 & 4.4 \\
\hline
\end{tabular}

Table 2.2: Applied magnetic field variation with distance above the anode surface and applied field frequency for the $6^{\prime \prime}$ stainless steel anode. The amplitude of the current in the coils was fixed at 10.0 A peak.

periments, the magnetic field value at a particular frequency was interpolated from the above measurements. The magnetic field amplitude was taken to be the value at the location of the cathode, interpolated from the above measurements, and assumed uniform along the arc length. The error in making this spatially uniform field assumption is shown to be negligible in Section 3.3.7.

\subsubsection{Arc stability experiments}

Cathode geometry dependence was an important parameter for the arc instability studied, as will be discussed in Chapter 4. Various types of cathode geometries were used in arc stability experiments. Photos of some of the cathodes are shown in Figure 2.8. Photos of cathodes taken after experiments are shown in Figure 2.9 . 


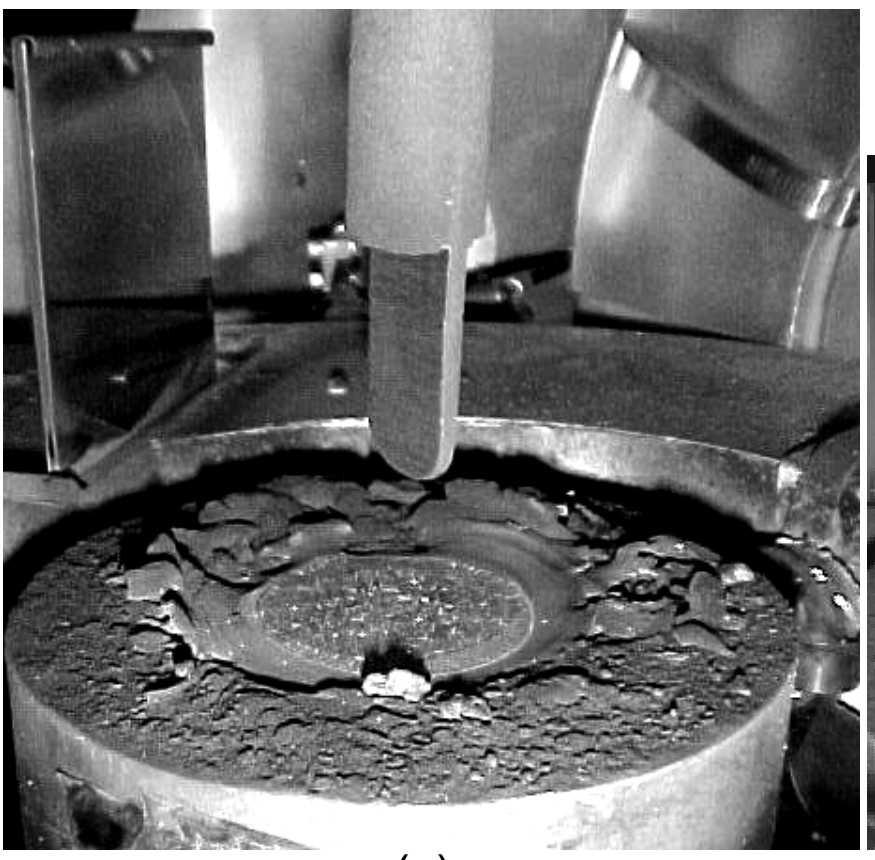

(a)

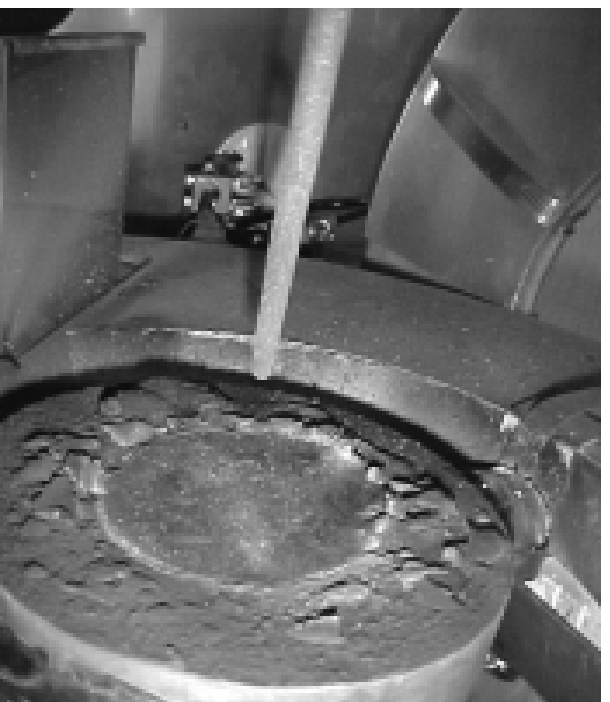

(b)

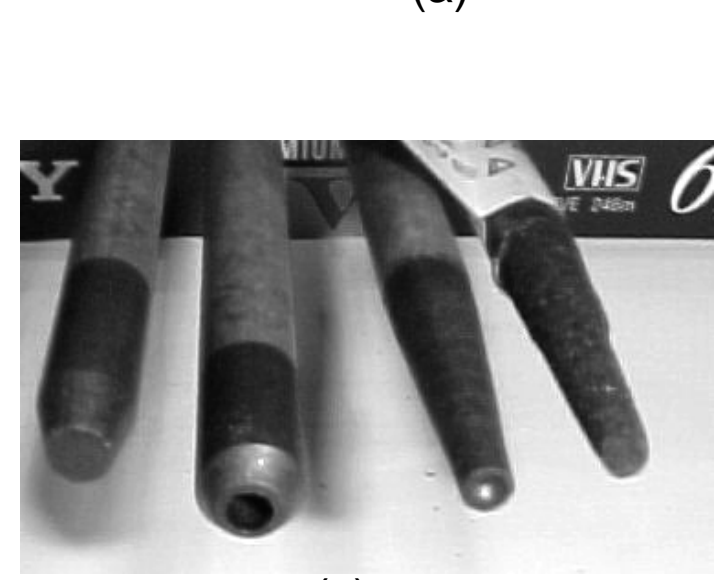

(c)

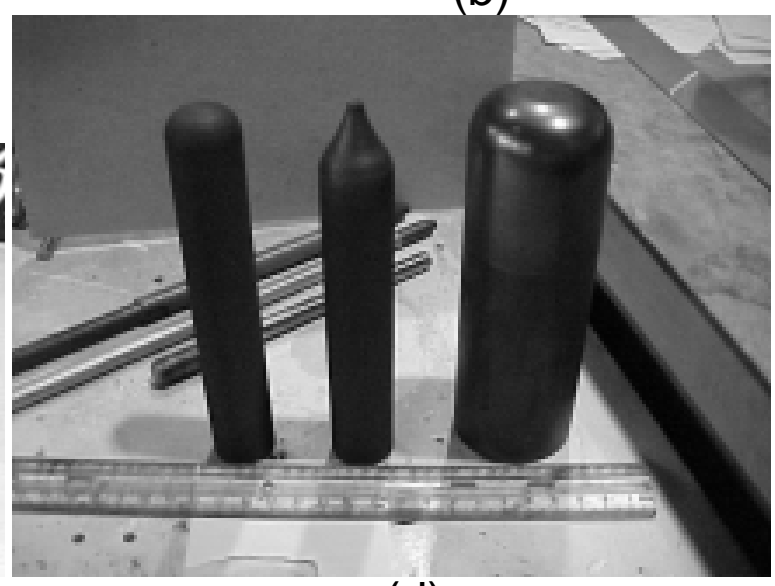

(d)

Figure 2.8: Photos of some of the cathodes used to study the dependence of arc stability on cathode geometry. (a) A "paddle" shaped cathode (number 19 in Table 4.1) in place prior to experiments. Also visible is the $6^{\prime \prime}$ dia stainless steel anode, the $90^{\circ}$ view mirror (upper left) and a portion of the transverse magnetic field coil (upper right). (b) A used standard 3/8" cathode. Note that the camera was at an angle when the photo was taken. (c) 3/8" standard cathodes, left-toright: new, drilled, graded with concave tip, and used. (d) McMaster cathodes, left-to-right: $1^{\prime \prime}$ dia, rounded edge, graded $1^{\prime \prime}$ dia, and $2^{\prime \prime}$ dia rounded edge. 


\subsubsection{Safety practices in arc furnace operation}

In addition to the usual practices associated with operating an experimental electrical apparatus, arc furnace operation requires some special precautions, which are discussed briefly below.

\section{Radiation}

An atmospheric pressure carbon arc is a very intense light source, and even a short unprotected exposure to it can be damaging to the eyesight. The required eye protection is similar to high-current carbon welding and cutting and is specified by the ANSI spec. Z87.1-1989 [38] as welding shade number 14.

Ultraviolet radiation is an additional hazard, with uncovered skin exposure resulting in possible skin burn.

In order minimize the radiation hazards, the arc furnace was enclosed in aluminum sheets, with view ports covered by welding shade 14 glass or plastic.

\section{Heat}

Since on the order of $20 \mathrm{~kW}$ of DC power is deposited inside the arc furnace, a potential for burns and a fire hazard exist even for tens of minutes after the arc has been shut off. Use of flammable materials in the furnace enclosure was therefore minimized and a sandbox was placed underneath the anode stand. However, the integrity of the fiberglass struts and epoxy casing of the coils can be jeopardized if the operations are carried on for too long. In order to minimize this hazard, the arc furnace was operated for a maximum of approximately $15 \mathrm{~min}$ at a time. Additionally, a fume hood with an exhaust fan was used to facilitate heat removal as well as removal of any gases generated during operation. 


\subsection{Diagnostics}

\subsubsection{Arc voltage and current monitoring}

The voltage across the arc is measured using a 1:10 voltage divider circuit, with a DC and AC coupled outputs. The input resistance of the voltage divider circuit is $60 \mathrm{k} \Omega$. The ratio of the input voltage to the output voltage was tested to be $10 \pm 1 \%$. The $3 \mathrm{~dB}$ bandwidth of the DC coupled output is DC-350 kHz and of the AC coupled output is $0.5 \mathrm{~Hz}-350 \mathrm{kHz}$. The voltage is measured between the attachment point of the negative lead to the cathode rod and the attachment point of the positive lead to the bottom of copper plate on which the anode (with or without the crucible) is resting (see Figure 2.1).

Arc current is measured using a LEM model LT 500-S current probe with frequency response $\mathrm{DC}-150 \mathrm{kHz}$. The probe's accuracy is $\pm 1.5 \mathrm{~A}$.

\subsubsection{Monitoring of the applied magnetic field}

Knowledge of the amplitude, phase, and frequency of the current in the magnetic field coils was necessary for the study of the arc in applied magnetic field. These were monitored using a Fluke Y8100 DC/AC current probe with a frequency response of DC-1 kHz. For frequencies above $1 \mathrm{kHz}$, a Pearson Electronics wideband current transformer model 110 with a $0.1 \mathrm{~V} / \mathrm{A}$ gain was used.

Transverse magnetic field is also monitored directly using a B-dot coil mounted below the level of the anode with its axis parallel to the axis of the transverse magnetic field coils. The B-dot coil has a gain of $3.5 \times 10^{-5} \mathrm{~V} /$ Gauss/Hz.

\subsubsection{Uncollimated light monitor}

In order to monitor the general light emission level of the arc, a pair of photodiodes are positioned inside the arc furnace. The photodiodes together with their amplifier circuit are mounted on the same stage to which the cathode chuck is 
attached, and so are fixed w.r.t. the cathode as the cathode is raised or lowered. The photodiodes are Hamamatsu S1336-5BQ, and together with the amplifier circuit have a $3 \mathrm{~dB}$ frequency response of $\mathrm{DC}-80 \mathrm{kHz}$. The photodiodes are protected from saturation from the arc light emission with a glass neutral density filter. One of the photodiodes is blinded with a piece of steel shim stock and is used to monitor any noise pickup on the data acquisition system.

\subsubsection{Gated imaging of the arc}

The shape of the arc for the applied magnetic field and instability experiments is obtained using an intensified CCD camera (ITT 4577). The axis of the camera is angled slightly w.r.t the horizontal in order for the anode surface to be visible, but is otherwise parallel to the axis of the transverse magnetic field coils. The vertical angles used were $9^{\circ}$ and $14^{\circ}$ w.r.t the horizontal.

The camera is protected from the intense light of the arc by neutral density filters and the small aperture of the the iris in the objective, as well as by the short exposure time (typically $10 \mu \mathrm{sec}$ ).

In order to monitor the shape of the arc column in three dimensions, a stainless steel mirror was used to create a $90^{\circ}$ view of the arc in the field of view of the camera, in addition to the direct view. The location of the mirror and the camera are shown in the top-down view of the setup in Figure 2.2. An air stream parallel to the mirror surface was used to cool the mirror. The mirror had to be sufficiently thick $\left(1 / 16^{\prime \prime}\right)$ to prevent warpage due to heat, and had to be cleaned and replaced periodically to prevent excessive metal splatter and soot buildup.

The direct view in the camera as well as the view in the mirror were calibrated as follows. A pair of LED's was set in holes in a rectangular aluminum block, with each LED shining through a $1.4 \mathrm{~mm}$ dia pinhole. With the distance between the pinholes known, the field-of-view of the camera was calibrated by imaging the LED's positioned horizontally as well as vertically, both in direct view and in the

mirror. A count of pixels between the imaged pinholes gave the calibrated distance per pixel horizontally as well as vertically. 
The camera is gated by external pulses, typically $10 \mu \mathrm{s}$ in duration. Typically, one gate pulse is used for each field of the camera, with the images acquired by the frame grabber in the non-interlaced mode. The clock used for the gating pulses is the internal clock of the camera, measured to be $59.939 \mathrm{~Hz}$, or 59.939 fields/s. When two exposures of the same field were required, each field was gated twice by two $10 \mu$ s pulses separated by $2 \mathrm{~ms}$.

The camera image of the arc as captured by a frame grabber is processed to give the coordinates of the points along the center of the luminous arc column by finding the centroid of brightness along each horizontal line of pixels. The arc shape is defined as the locus of such points.

\subsubsection{Continuous arc position monitoring}

Continuous monitoring of the arc column position along a horizontal line is accomplished using a linear array of 8 individual optical fibers going into 8 separate photodiodes. The photodiodes are Hamamatsu S1336-5BQ, and together with their amplifier circuits have a $3 \mathrm{~dB}$ frequency response of $\mathrm{DC}-80 \mathrm{kHz}$. The optical setup for projecting an image of the arc onto the 8 fibers is shown in Figure 2.10 and is described as follows.

An image of the arc is focused onto a fiberoptic image bundle $(400 \times 400$ fibers) using an objective $(A)$ (Figure 2.10). The objective is located directly above the ITT camera. The fiberoptic bundle is brought to an optical table, whereupon the image of the arc is magnified using a lens $(B)$. The image of the arc formed by lens $(B)$ is focused in the plane of the 8 individual fibers, aligned horizontally. The final image magnification ( $1 \mathrm{~cm}$ at fibers $=3.3 \mathrm{~cm}$ at arc) was chosen so that the typical horizontal displacement of the arc due to the instability or application of the transverse magnetic field would be within the line segment covered by the fiber array.

The response of each of the fibers as registered by the corresponding photodiode is calibrated relative to the others as follows. A cylindrical lens in front of the fibers was used to form a line image of a flashlight onto the fiber array. The resulting voltages were digitized and normalized to the highest one. The normalized 
voltages are used as calibration weights to compensate for any inequities in gain of the individual fibers and photodiodes.

The information on the arc column position along the horizontal line defined by the fiber array is at each point in time is obtained as follows. A Gaussian profile is fitted to the calibrated voltages of the 8 photodiodes. The fit parameters are the center and the width of the Gaussian. The center of the Gaussian is taken to be the location of the center of the arc column. The details of the actual emission profile of the arc are not important, since only the location of the center of the profile is required. The fit fails, however, on the occasions when the image of center of the column swings out past the last fiber on either side.

\subsection{Data Acquisition}

Data acquisition is accomplished using a National Instruments PCI-6017E data acquisition board on a PC. The control of the data acquisition and processing is done using LabVIEW ${ }^{\mathrm{TM}}$ graphical programming language. Data is sampled typically at $20 \mathrm{kSamples} / \mathrm{sec}$.

The trigger for the data acquisition board and the frame grabber for the camera image acquisition is obtained as follows. The camera is being gated continuously with typically $10 \mu$ s pulses at $59.939 \mathrm{~Hz}$, as discussed above. A manual trigger pulse sets a flip-flop circuit to output a logic "1". The next camera gating pulse resets the flip-flop to a logic " 0 ", triggering the data acquisition board and the frame grabber. Data acquisition thus starts precisely at the moment the first acquired image field is gated. 


\subsection{Arc characteristics}

\subsubsection{Static characteristics}

As mentioned above, the power to the arc is supplied using a power supply operated in the current-controlled mode. For all the measurements presented below, a particular current value was dialed up on the current-controlled arc power supply, then a data sample at that current was taken.

The measured variation of the arc voltage with arc length for various arc currents is shown in Figure 2.11. As can be seen from the figure, the arc voltage increases linearly with the arc length. This is expected from the approximately constant cross-section and temperature (and thus resistivity) along the arc column, and has been found in previous experiments [9].

Since the voltage across the arc varies approximately linearly with the arc length, the voltage drop in the electrode sheaths can be estimated by extrapolating the fits in Figure 2.11 to zero arc length. The sum of voltage drops in the cathode and anode sheaths thus obtained is plotted in Figure 2.12. As can be seen, the sum of the voltage drops in the electrode sheaths is approximately constant in this current range. This is similar to the observations given in Ref. 12 for argon arcs on tungsten electrodes up to $200 \mathrm{~A}$, in which the cathode fall is found to vary by approximately $2 \mathrm{~V}$ each in the range 50-200 A.

The electric field in the arc column, taken as the slope of the fits in Figure 2.11, is plotted vs. the arc current on the right-hand scale of Figure 2.13. The same plot shows the variation of the total voltage across the arc measured during a different set of runs (left-hand scale). The cathode position was not changed for the voltage data. The electrode gap did vary somewhat, however, due to the cathode erosion. To counter this, the data points at the several currents were taken out-of-sequence.

Both the column electric field and the total arc voltage show a minimum at approximately $140 \mathrm{~A}$ and have similar variation with current. A possible inference from the plot is that the total arc voltage varies with current due to the variation 
of the electric field of the column, and not of the sheath voltages. This is consistent with the data in Figure 2.12 showing that the voltage drop across the electrode sheaths is approximately constant in this current range.

The arc thus has a rising voltage-current characteristic above approximately $140 \mathrm{~A}$. The falling characteristic below $\approx 140 \mathrm{~A}$ would not imply that the arc would be electrically unstable, since the power supply in the present setup is run in a current-controlled mode.

\subsubsection{Conductivity temperature of the arc plasma}

Since the electric field in the arc column has been measured, the average conductivity of the plasma can be estimated and an estimate of the plasma temperature obtained.

Assuming that the conduction radius of the column of $\approx 0.3 \mathrm{~cm}$ at $200 \mathrm{~A}$, equal to the radius of the bright central core, the current density $J$ in the column is:

$$
J=\frac{200 \mathrm{~A}}{\pi\left(3 \times 10^{-3} \mathrm{~m}\right)^{2}}=7.1 \times 10^{6} \mathrm{~A} / \mathrm{m}^{2}
$$

Using the value of electric field at 200 A from Figure 2.13 of $9 \mathrm{~V} / \mathrm{cm}$, the resistivity is:

$$
\eta=E / J=\frac{900 \mathrm{~V} / \mathrm{m}}{7.1 \times 10^{6} \mathrm{~A} / \mathrm{m}^{2}}=1.3 \times 10^{-4} \Omega \cdot \mathrm{m}
$$

Assuming Spitzer resistivity model [39],

$$
\eta=5.2 \times 10^{-5} \frac{Z \ln \Lambda}{T^{3 / 2}} \Omega \cdot \mathrm{m}, T \text { in } \mathrm{eV}
$$

for $Z=1$ and $\ln \Lambda=5$, obtain $T=1.6 \mathrm{eV}$, or $19,000 \mathrm{~K}$. This temperature is higher than the 10,000-12,000 K measured for a 200 A carbon arc by Maecker [40]. The Spitzer model, however, is probably not fully applicable to the arc plasma because the plasma is not fully ionized. According to numerically computed tables 
of conductivity of air vs. temperature in Ref. 41, the above resistivity value occurs at a temperature of $14,900 \mathrm{~K}$, which is closer to the Maecker measurements.

\subsubsection{Dynamic characteristics}

The dynamic, or small-signal AC, voltage-current characteristics discussed below are observed for arcs which are oscillating on a fast time scale, either during AC transverse magnetic field application or during arc instability. The characteristics are obtained during a single data sample, with the average current value fixed.

The dynamic characteristics appear to be similar for the arc in either the applied transverse AC magnetic field, a low amplitude unstable arc, or large amplitude instability. Typical V-I characteristics for the three cases with the non-inductive ballast resistor are plotted in Figures 2.14, 2.15, and 2.16. In all three cases, the characteristics are negatively-sloping, with falling current and rising voltage and vice versa.

The similarity in behavior in the three cases suggests that the characteristics are determined by the response of the power supply to the changing load at the arc, and can be understood as follows. The EMHP 80-250 power supply contains a $0.21 \mathrm{~F}$ capacitor bank across the output. As the arc length changes due to deflection by the applied transverse magnetic field (Section 3.3.3) or due to the instability (Section 4.3.3), the voltage across the arc changes. If the arc circuit includes a ballast resistor $R$, as shown in Figure 2.6, the voltage across the arc, $V_{\text {arc }}$, and the voltage across the power supply, $V_{s}$ are related as:

$$
V_{\mathrm{arc}}=V_{s}-I R
$$

On the time scale shorter than the $R C$ time of the capacitor bank and the arc load,

$$
\tau=R C=(60 \mathrm{~V} / 200 \mathrm{~A})(0.21 \mathrm{~F})=(0.3 \Omega)(0.21 \mathrm{~F}) \approx 60 \mathrm{msec},
$$

the voltage across the capacitor bank, and therefore, at the output of the power supply would remain constant $\left(V_{s}=\right.$ const.). Under this condition, $V_{\text {arc }}$ is related 
to $I$ as shown in Figure 2.17. Therefore, from Eq. (2.4), any fast change in the arc voltage would result in a change in the arc current with $\Delta V_{\operatorname{arc}}=-R(\Delta I)$. Indeed, for the three cases considered in Figures 2.14, 2.15, and 2.16, the values of $\Delta V_{\text {arc }} / \Delta I$ are $-0.14 \Omega,-0.12 \Omega$, and $-0.13 \Omega$. The measured value of the ballast resistor used for the runs shown was $0.12 \pm 0.01 \Omega$.

The presence of a "hysteresis" in the characteristic in Figure 2.14 could be explained by the presence of inductance due to the current leads in the arc circuit. Adding an equivalent inductance $L$ in series with the arc, modifies Eq. (2.4) as follows:

$$
V_{\mathrm{arc}}=V_{s}-I R-L \frac{d I}{d t}
$$

Assuming a sinusoidal variation of the arc voltage (due to arc length change) about the DC operating point $\left(V_{0}, I_{0}\right)$, write:

$$
\begin{gathered}
V_{\mathrm{arc}}=V_{0}+V_{1} e^{i \omega t} \\
I=I_{0}+I_{1} e^{i \omega t}
\end{gathered}
$$

Substituting the above expressions into Eq. (2.6) and solving for I:

$$
I=I_{0}-\frac{V_{1} e^{i\left(\omega t-\tan ^{-1}(\omega L / R)\right)}}{R \sqrt{1+(\omega L / R)^{2}}}
$$

The inductance of the $\approx 7 \mathrm{~m}$ long leads of the arc circuit is $L \approx 7 \mu_{0}=9 \times 10^{-6} \mathrm{H}$. The voltage-current characteristic dictated by Eq. (2.9) is plotted in Figure 2.18, with the values for $V_{0}, V_{1}$, and $I_{0}$ taken from the example in Figure 2.14, with $\omega=2 \pi(2 \mathrm{kHz})$ (the arc length varies at twice the applied magnetic field frequency, as discussed in Section 3.3.3). As can be seen from the similarity of Figures 2.18 and 2.14, the inductance in the arc circuit can explain the "hysteresis" seen in the dynamic V-I characteristics. 

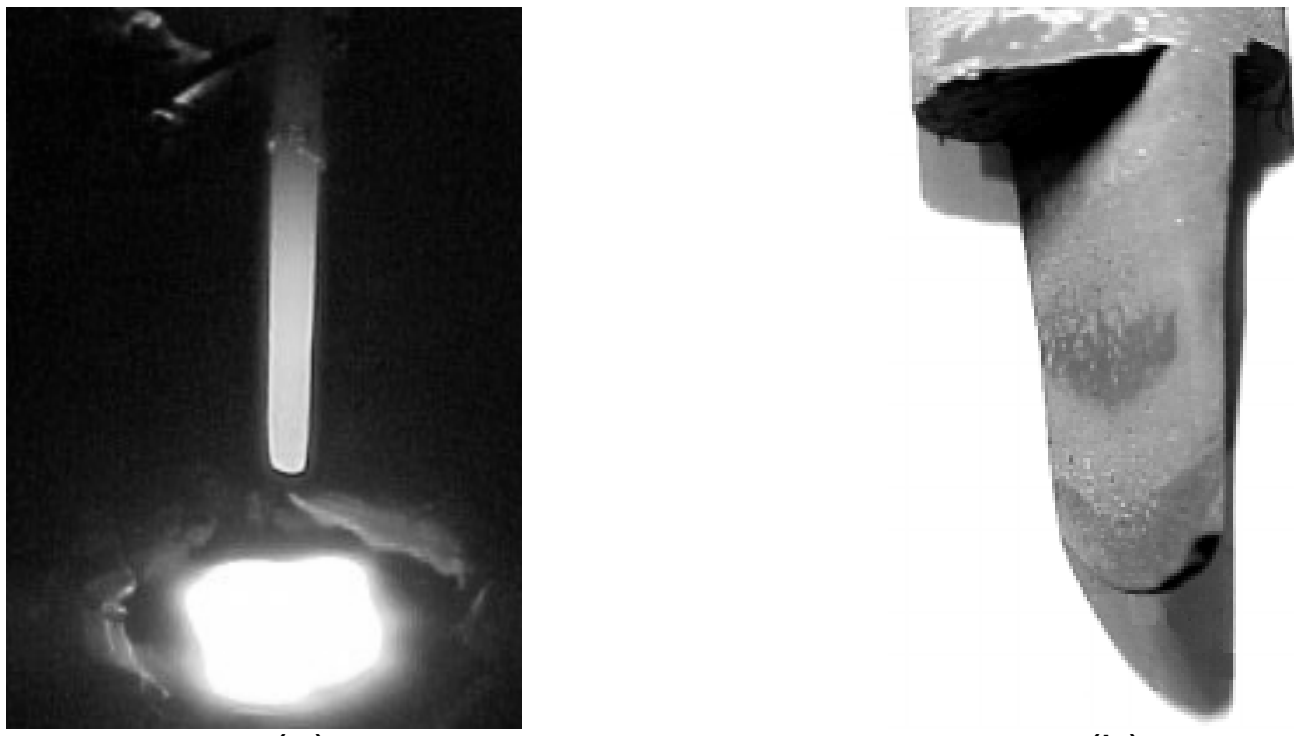

(a)

(b)

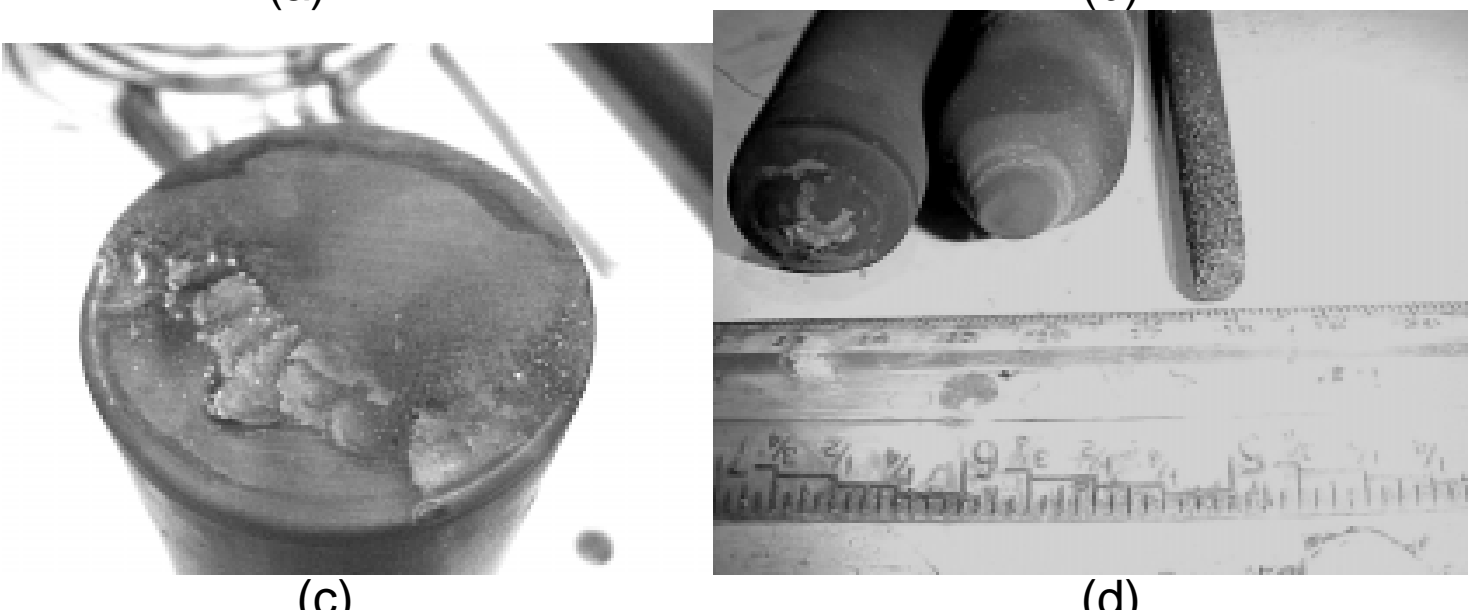

(c)

(d)

Figure 2.9: Photos of cathodes after experiments: (a) The cathode and anode glowing after the arc shut-off. (b) The "paddle" shaped cathode shown before in Figure 2.8(a). During the experiments, the arc was attached to the tip and slowly moving back-and-forth along it. (c) The surface tip of a $2^{\prime \prime}$ dia cathode removed after running for $\approx 10 \mathrm{sec}$. Most of the time the arc was attached at the edge and was moving slowly along it. Note how the initially sharp edge is eroded after a single pass of the arc. Metal deposits apparently from the anode vapor are also visible on the surface. (d) $1^{\prime \prime}$ dia cathodes and a standard $3 / 8^{\prime \prime}$ dia cathode after arcing. 

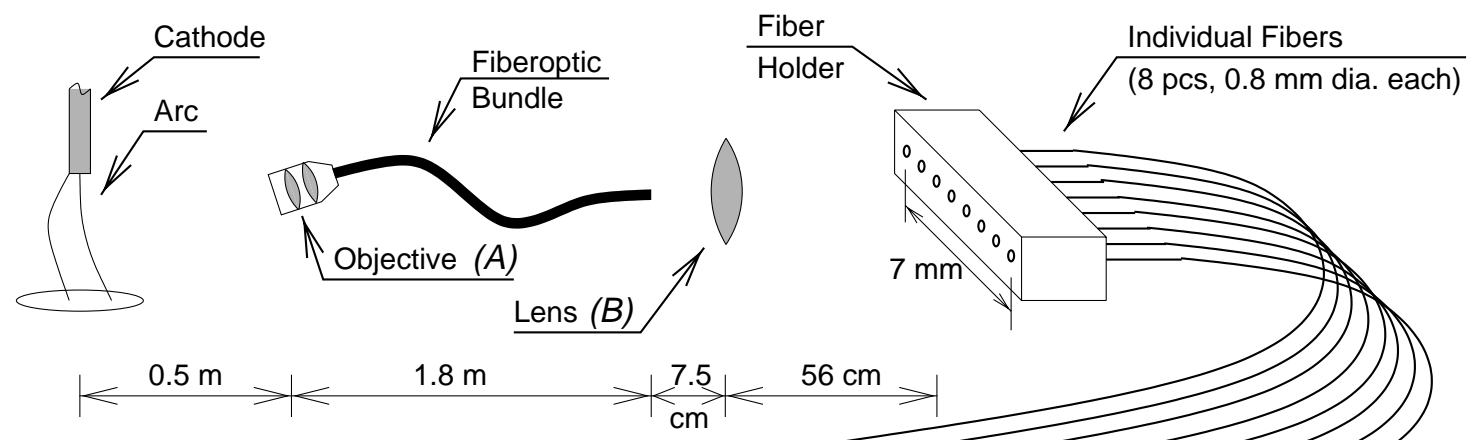

Note: Drawing not to scale.

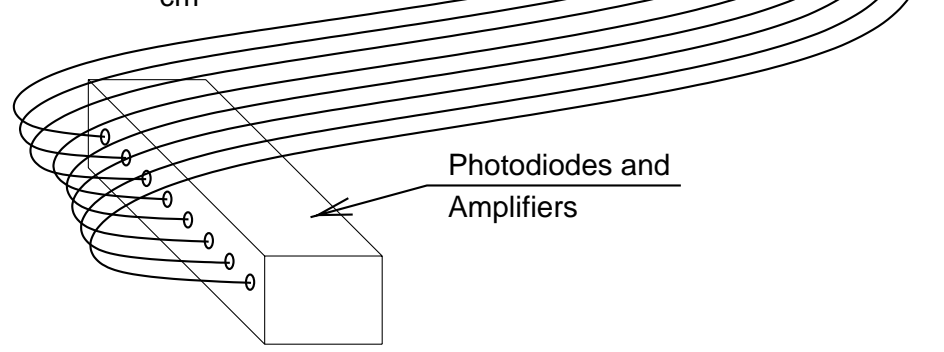

Figure 2.10: A schematic illustrating the collimated photodiode array setup for continuous monitoring of the arc column position. The schematic is not drawn to scale.

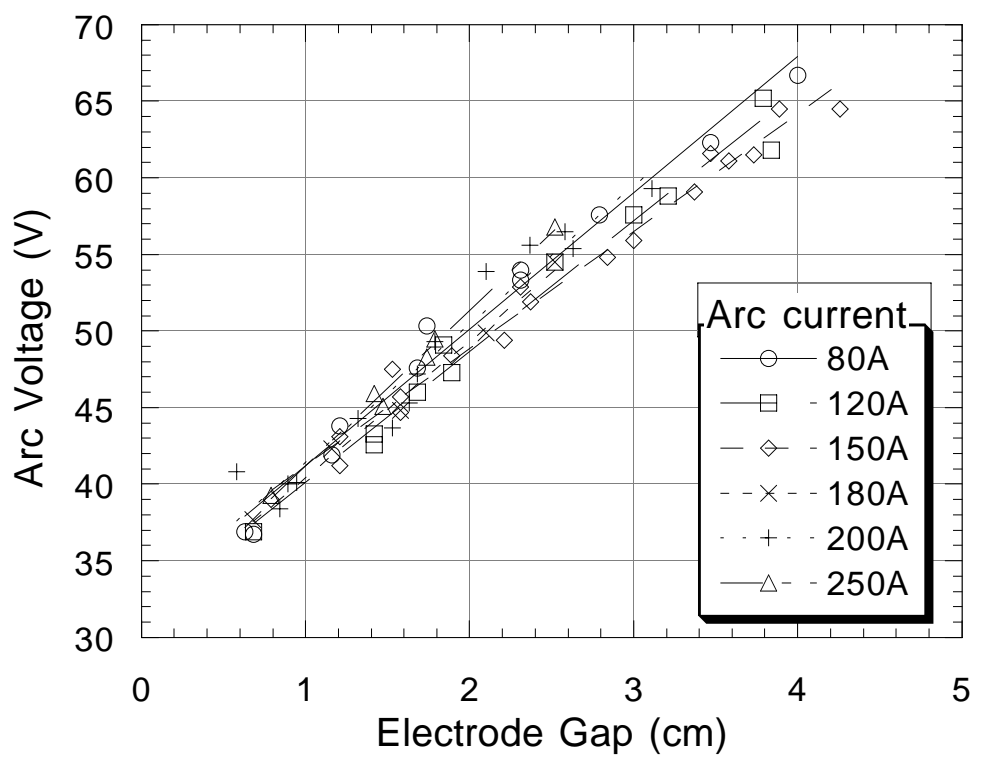

Figure 2.11: Arc voltage variation with electrode gap for several arc currents. Points with RMS fluctuation level $\geq 5 \%$ were excluded. The voltage increases linearly with electrode gap, indicating that the electric field is constant in the arc column, as expected [9]. 


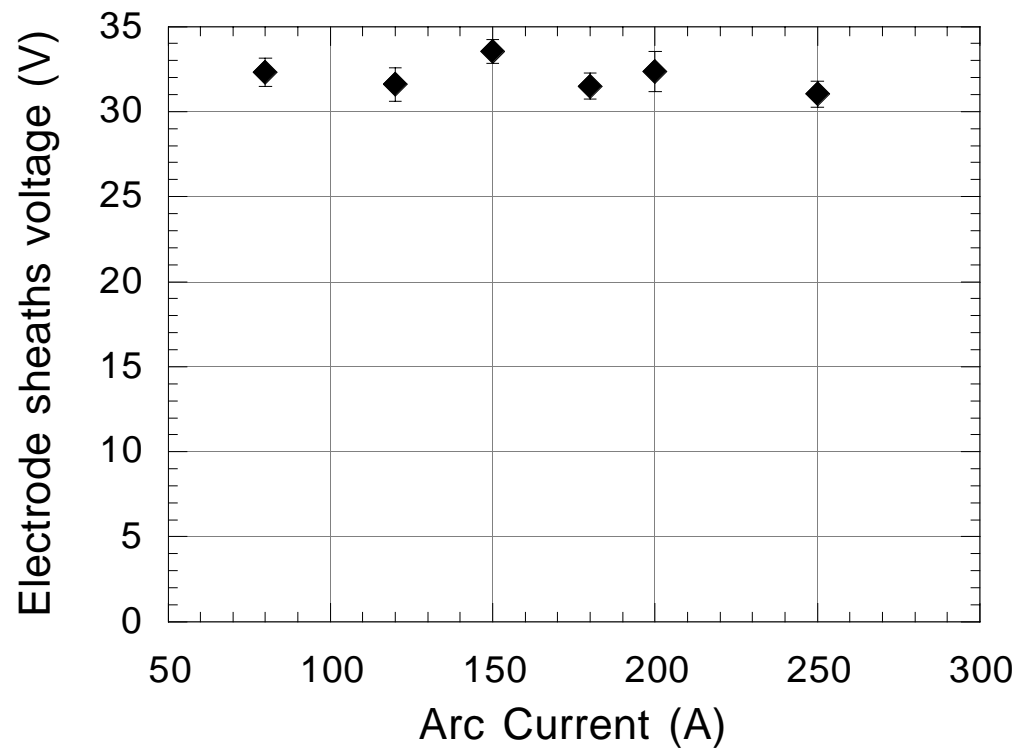

Figure 2.12: The total voltage drop in the cathode and anode sheaths is approximately constant in the current range studied. The voltage drop is found by extrapolating the voltage vs. electrode gap fits in Figure 2.11 to zero gap. The error bars are estimated from the standard errors in the least-squares fits. 


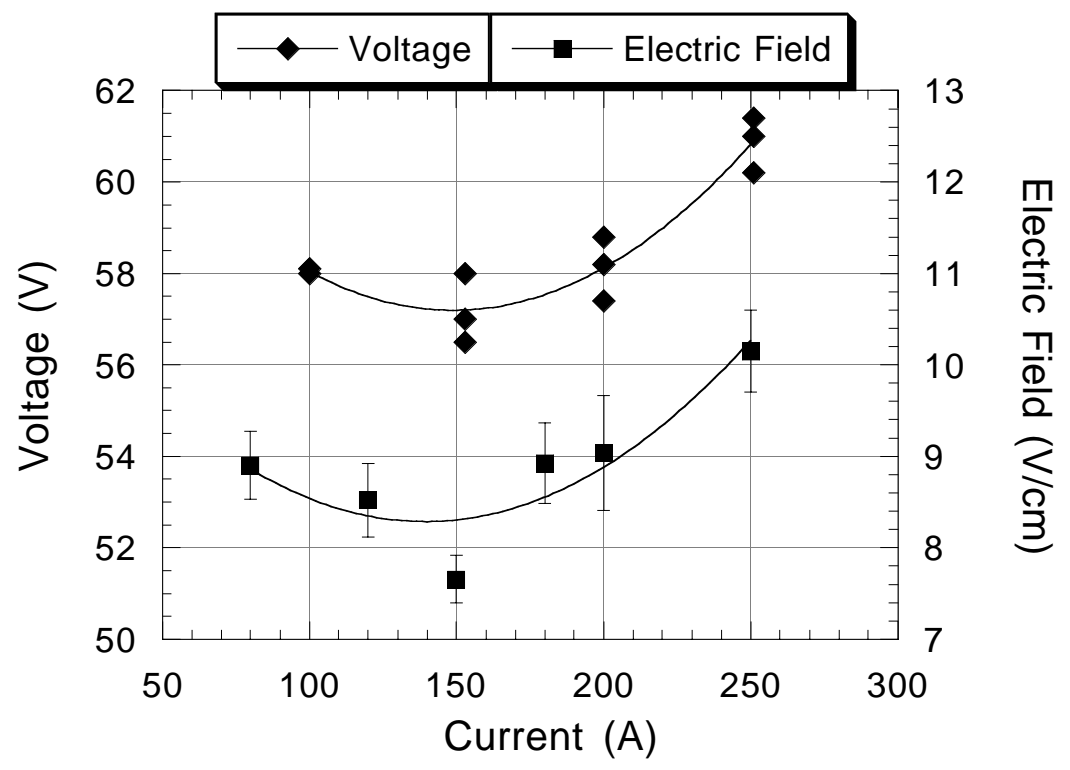

Figure 2.13: Arc column electric field variation with the arc current (right-hand scale). Also shown is a quadratic polynomial fit to the data. The electric field is taken as the slope of the fits in Figure 2.11. The error bars are estimated from the standard errors in the least-squares fits. The variation of the total voltage across the arc measured during a different set of runs is plotted on the left-hand scale. A quadratic polynomial fit to the data is also shown. The electrode gap for the voltage data is $2.5 \pm 0.2 \mathrm{~cm}$, with the variation due to cathode erosion. 


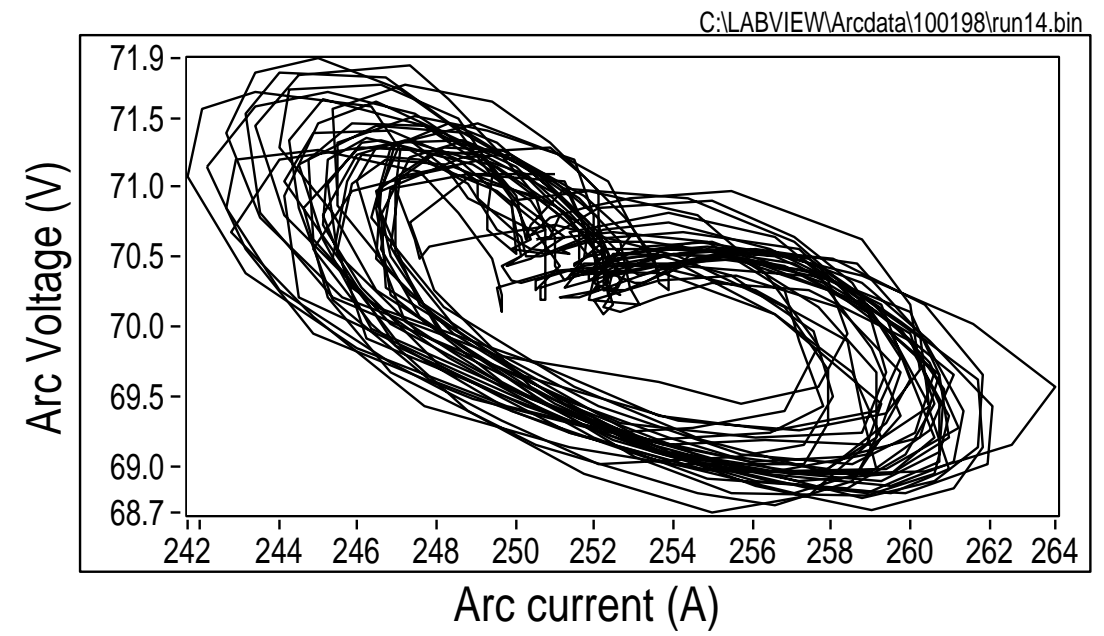

Figure 2.14: Typical small-signal AC V-I characteristics for a stable arc in transverse magnetic field. The applied field frequency is $1 \mathrm{kHz}$; the average arc current is $251 \mathrm{~A}$. As can be seen, the voltage and current variation is nearly $180^{\circ}$ out of phase (exactly $180^{\circ}$ would be a line with a negative slope). This phase relationship also evident on the plot of waveforms themselves, shown in Figure 3.20.

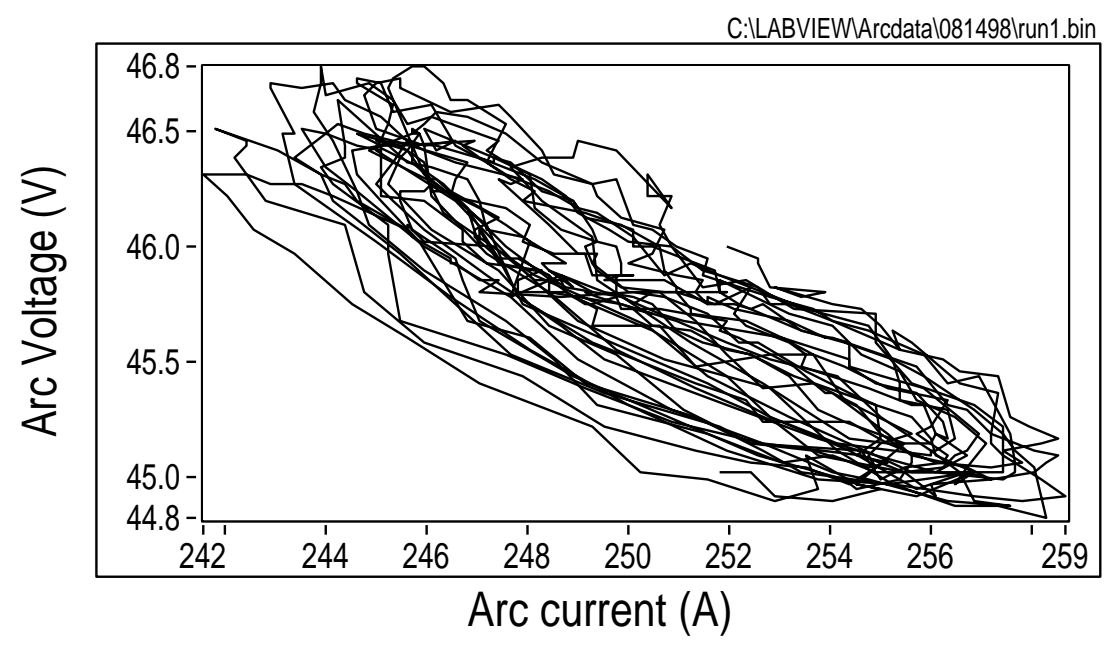

Figure 2.15: Typical small-signal AC V-I characteristics for low amplitude instability. Average arc current is $251 \mathrm{~A}$. The voltage and current variation is nearly $180^{\circ}$ out of phase, similar to that for applied AC magnetic field shown above in Figure 2.14. The $180^{\circ}$ out-of-phase relationship is also evident on the instability waveforms themselves, shown in Figure 4.4. 


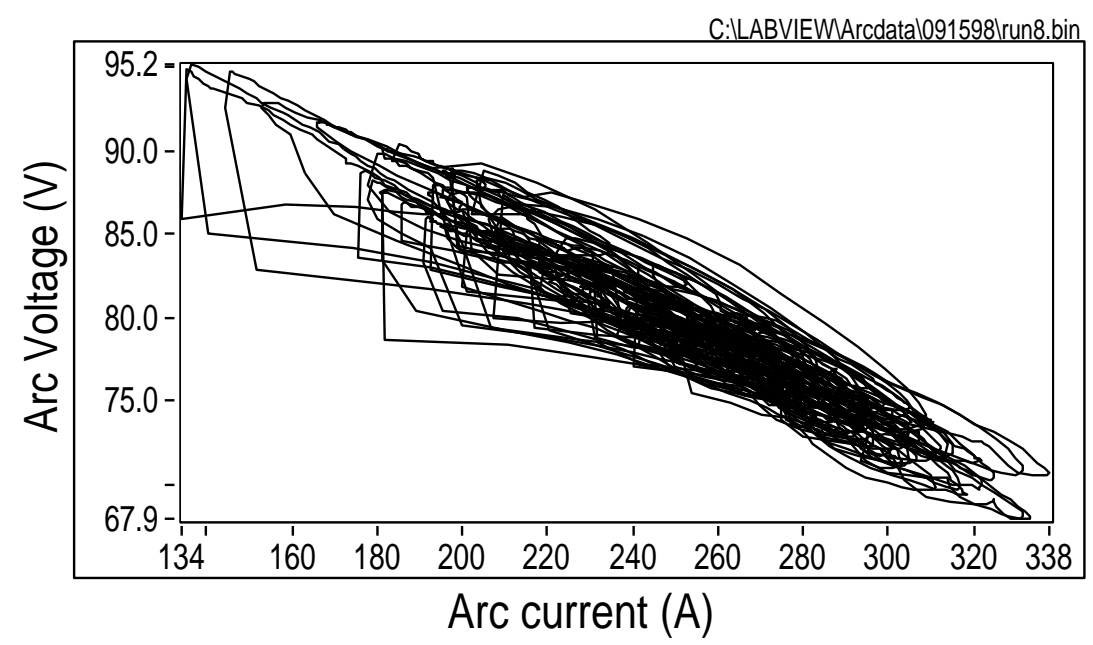

Figure 2.16: Typical AC V-I characteristics for large amplitude instability. The average arc current is $251 \mathrm{~A}$. Note the large amplitude of the current variation. The voltage and current variation is still nearly $180^{\circ}$ out of phase, similar to that for applied AC magnetic field and low amplitude instability.

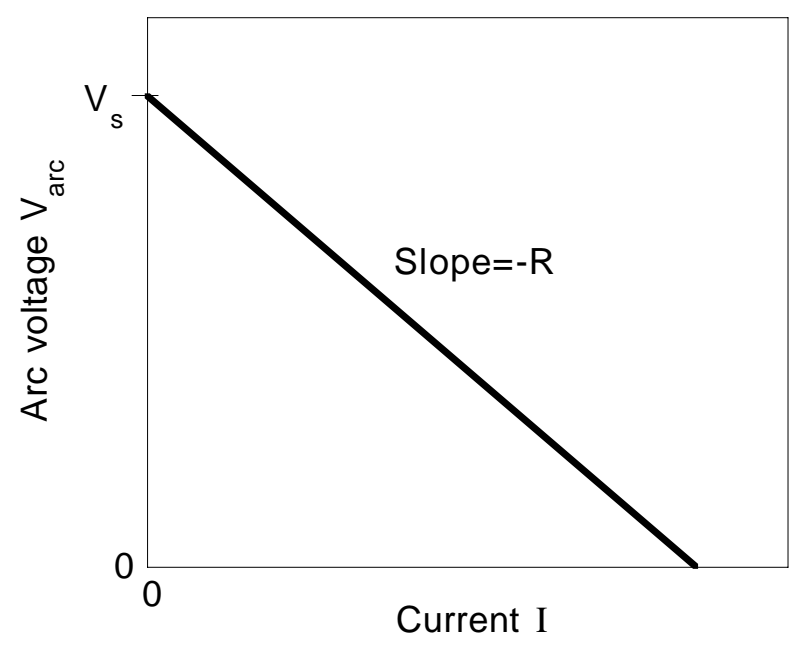

Figure 2.17: Plot of Eq. (2.4) for constant power supply voltage $V_{s}$. The arc voltagecurrent characteristic has negative slope of $-R$, where $R$ is the ballast resistance value. 


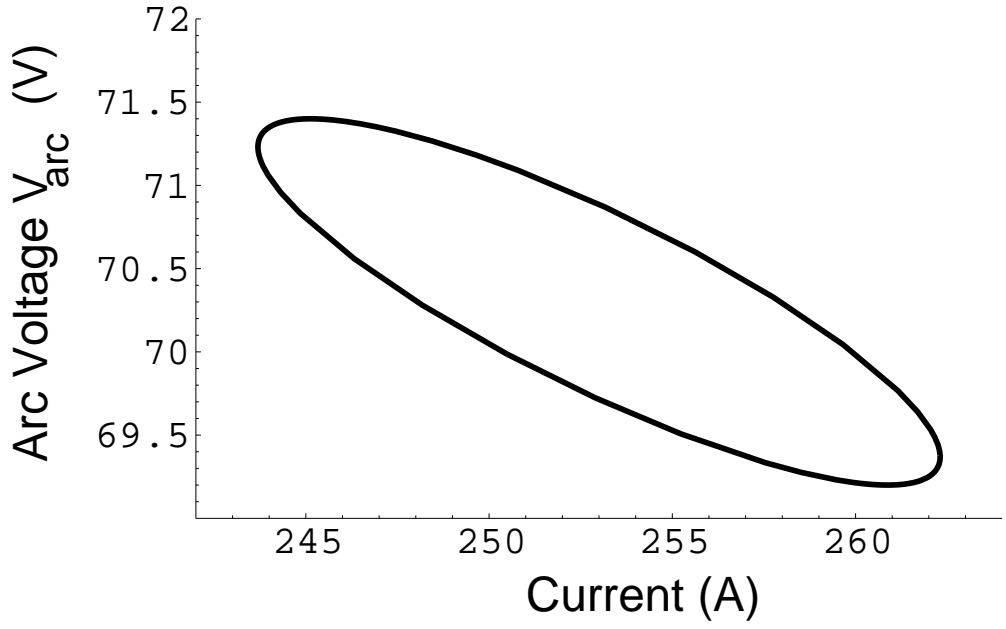

Figure 2.18: Plot of Eq. (2.9) for constant power supply voltage $V_{s} . R=0.12 \Omega$, $L=9 \times 10^{-6} \mathrm{H}$, and frequency is $2 \mathrm{kHz}$. 


\section{Chapter 3}

\section{Arc Deflection in Transverse Magnetic Field}

This Chapter pertains to dynamics of the arc forced perpendicular to the arc current by the application of an external magnetic field. As will be seen below, the resulting effect on the arc is a stable and orderly motion. Occurrence of instability is discussed and analyzed in the following Chapter.

The Chapter is organized as follows: Section 3.1 describes the experimental setup, Section 3.2 gives the experimental results, Section 3.3 gives a derivation of the model, Section 3.4 applies the model to the experimental data, Section 3.5 projects the model to an industrial scale arc furnace, and finally, Section 3.6 discusses the results and gives conclusions.

\subsection{Experimental Setup}

The general description of the arc furnace setup is given above in Chapter 2 . The details of the setup pertaining to the deflection experiments is described here.

In the arc deflection experiments, a set of coils is used to apply a field to the arc in the direction perpendicular to the arc current, as described in Chapter 2. Deflection experiments are conducted using the $3 / 8$ " graphite rod cathode described 
in Chapter 2, as well as a larger 1" diameter graphite cathode. Two different types of anodes were used: a large area iron ( $4^{\prime \prime}$ diameter) cylinder enclosed in a conducting tar impregnated $\mathrm{MgO}$ brick crucible (such as used in arc furnaces), and non-magnetic stainless steel ( $6{ }^{\prime \prime}$ diameter) ingots. There was no difference in the arc behavior between these two types of anodes.

The parameter space available in the experiments is arc length $0-7 \mathrm{~cm}$, arc current 100-250 A, applied magnetic field strength 0-5 Gauss and frequency DC$2 \mathrm{kHz}$.

The shape of the arc in applied magnetic field is obtained using an intensified CCD camera (ITT 4577) with typically a $10 \mu \mathrm{sec}$ gate duration at a rate of 60 fields/sec (non-interlaced). The plane of view of the camera is perpendicular to the applied magnetic field. The raw image of the arc as captured by a frame grabber is processed to give the coordinates of the points along the center of the luminous arc column by finding the centroid of brightness along each horizontal line of pixels. The arc shape is defined as the locus of such points.

Continuous monitoring of the arc column position along a horizontal line is accomplished using a linear array of 8 collimated photodiodes with a bandwidth of $80 \mathrm{kHz}$.

Data acquisition is synchronized to the gating pulse of the camera. The image capture and data acquisition systems are described in more detail in Chapter 2.

\subsection{Description of Experimental Results}

\subsubsection{Arc behavior with no external magnetic field}

The starting point for all the applied transverse magnetic field experiments is an arc with less than $1 \%$ voltage and current fluctuations that is stable except for a slow precession of the arc on the tip of the cathode. The effect of this motion is that the angle the arc axis makes with the axis of the electrodes varies within approximately $\pm 5^{\circ}$. Since the time scale for this precession (1 cycle in $20 \mathrm{sec}$ ) is much longer than all other time scales in the experiment, this angle is taken as a DC 
position offset in the analysis below. The origin of this precession lies in thermal and erosion processes at the cathode and will be discussed more in Section 4.4.2.

\subsubsection{Observed response of the arc}

Motion of the arc in response to applied transverse magnetic field is strictly in the plane perpendicular to the field direction. This was ascertained by viewing the arc directly as well as at $90^{\circ}$ using a mirror.

No significant differences in the response of the arc are found between $3 / 8^{\prime \prime}$ and $1^{\prime \prime}$ diameter graphite cathodes and between iron and steel anodes.

\section{DC and low frequencies}

For DC applied magnetic field, the arc exhibits a deflection in the direction expected from the $\vec{J} \times \vec{B}$ force, with maximum deflection at the anode (Figure 3.1). The shape of the deflected arc is stable and is well fitted by a parabola. For
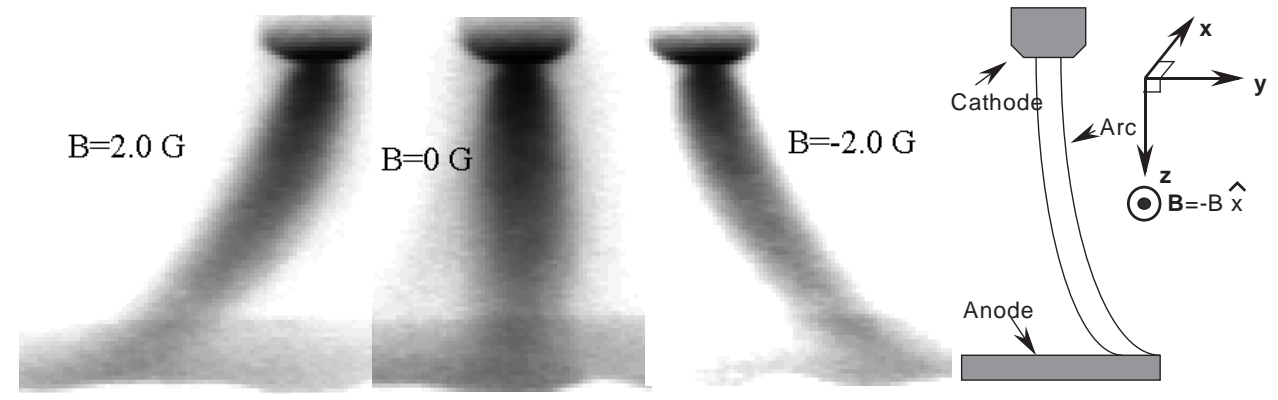

Figure 3.1: Arc deflection for DC and low frequency AC magnetic field. Pictures are negatives of images taken with $10 \mu$ sec exposure intensified CCD camera. Pictures are compressed vertically $2.4: 1$. The schematic shows the location of the cathode and the anode and the direction of applied magnetic field.

low frequency oscillating transverse magnetic field, when the applied frequency is $\lesssim 80 \mathrm{~Hz}$, the arc is swept across the anode surface while being anchored at the cathode. The shape of the arc at any point in time is parabolic, as in the DC field case. The amount of deflection and the arc shape are found to be independent of 


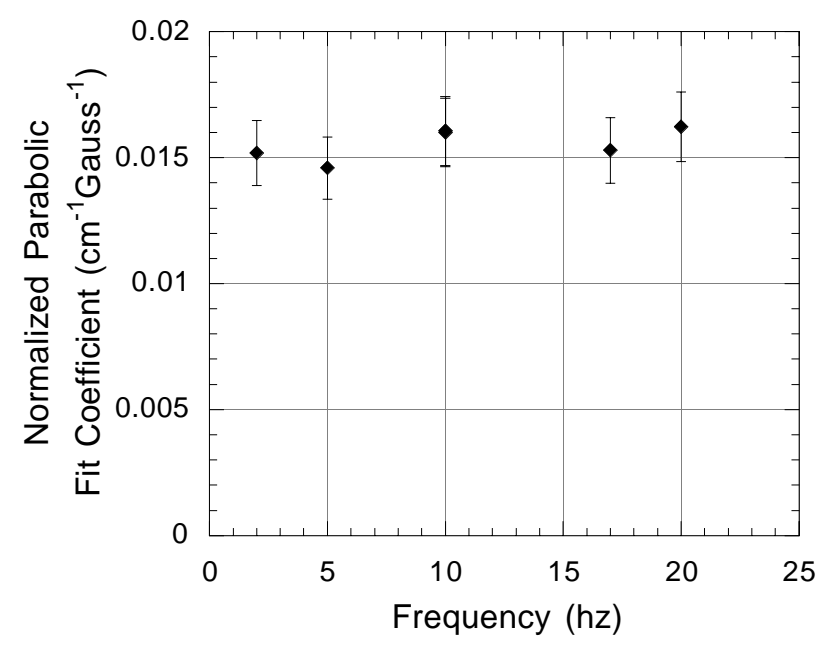

Figure 3.2: The shape of the arc is independent of frequency for low frequency applied magnetic field. Here, the coefficient $A_{2}$ resulting from the fit of a parabola $\xi(z)=A_{1} z+A_{2} z^{2}$, where $\mathrm{z}$ is the vertical distance from the cathode, to the arc shape, normalized to instantaneous magnetic field is plotted vs. frequency. Arc current is $145 \mathrm{~A}$; arc length is $4 \mathrm{~cm}$. The error bars are the standard error from linear fits of data at different phases of the applied field.

magnetic field frequency for low frequencies. This can seen in Figure 3.2 showing the results of parabolic fit to arc shape vs. frequency.

Figure 3.2 is obtained by fitting a parabola $\xi(z)=A_{1} z+A_{2} z^{2}$, where $\mathrm{z}$ is the vertical distance from the cathode and $\xi$ is the measured displacement from the vertical, to the arc shape for different frequencies of the applied field. The coefficient $A_{1}$ accounts for the variation of the angle of the arc w.r.t. the vertical (discussed in Section 3.2.1 above). For each frequency, the fits are done at several different phases of the applied magnetic field. The resulting $A_{2}$ coefficients vary linearly with the instantaneous applied magnetic field. Effects of any DC bias magnetic field, such as due to Earth's magnetic field and the field from the current feeds, are removed by performing a linear fit of the $A_{2}$ vs. the instantaneous applied magnetic field. Each data point in Figure 3.2 is the mean value of the slope thus obtained. 


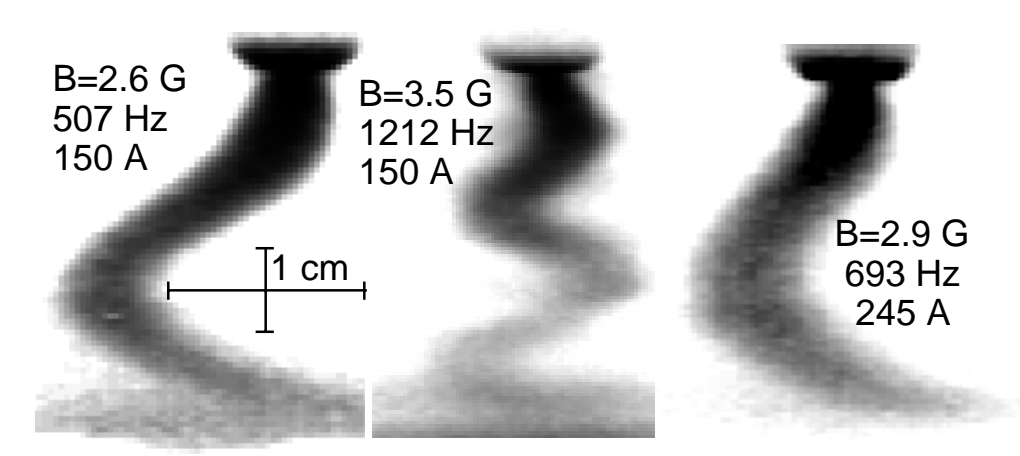

Figure 3.3: Sample pictures of the arc in applied AC magnetic field. (Negatives, $10 \mu \mathrm{sec}$ exposure; compressed vertically 2.4:1). The geometry is the same as in Figure 3.1. Labels indicate the peak amplitude and frequency of applied magnetic field and the arc current. As can be seen from the pictures, the mode number is higher for higher frequency and lower for higher current.

\section{High frequencies}

For applied magnetic field frequencies above $100 \mathrm{~Hz}$, the arc shape is no longer parabolic. As in low frequency case, the arc is anchored at the cathode and is swept across a line segment of the anode. However, the arc shape at any moment in time is a growing sinusoid, with the amplitude increasing towards the anode. Sample images of the arc at three different frequencies of applied field are shown in Figure 3.3. The longitudinal mode number increases with the applied frequency, while the amplitude of displacement at a given vertical position decreases with increasing frequency. For higher arc currents, the mode number decreases. The arc shape is periodic in time at the frequency of the applied field. The analysis of the high frequency data is in Section 3.4.2.

\subsection{Theoretical Model}

The arc shape data for DC and $\mathrm{AC}$ applied magnetic fields was quantitatively analyzed on the basis of a model derived below. Comparison of data with the model is given in Section 3.4. 


\subsubsection{Assumptions}

In order to make an analytical solution tractable, a one-dimensional (1-D), linear approximation is used. The following assumptions are made in the derivation:

1. Arc dynamics can be described by single fluid equations due to the high collisionality, as discussed in Section 3.3.8.

2. A thin-wire (1-D) approximation is made replacing actual cross-sectional profiles of mass density, current density, and axial flow velocity, with linear mass density, arc current, and an average jet speed and that are assumed constant along the length of the column. Any pressure gradients are assumed to be internal to the arc.

3. The arc displacement from the equilibrium axis is sufficiently small for transverse component of current density and jet velocity to be small compared to the respective axial components.

4. Motion of the arc due to heat flux effects [42] is negligible (see Section 3.3.8).

5. The self-magnetic field effect is negligible. This assumption is relaxed in Section 3.3.6.

6. The arc is anchored at the cathode emission spot and is free to move over the anode surface. This assumption is relaxed in Section 3.3.4.

7. Gravity effects are neglected. The characteristic flow speed due to buoyancy is $\sqrt{g L} \sim 0.8 \mathrm{~m} / \mathrm{s}$, where $g$ is acceleration of gravity and $L \leq 0.07 \mathrm{~m}$ is the arc length; it is much slower than the average jet speeds inferred $(\sim 20 \mathrm{~m} / \mathrm{s})$.

8. The applied magnetic field is uniform along the length of the arc. This assumption is examined in Section 3.3.7. 


\subsubsection{Derivation of Equation of Arc Shape}

With the above assumptions, the force balance equation is:

$$
m \frac{\partial \vec{v}}{\partial t}+m(\vec{v} \cdot \nabla) \vec{v}=\vec{I} \times \vec{B}
$$

where $m$ is the linear mass density. Letting z-axis be the equilibrium arc axis, $\vec{B}=\hat{x} B \cos (\omega t)$, jet velocity $\vec{v}=\tilde{v} \hat{y}+v \hat{z}$, where $v$ is the jet speed and $\tilde{v}$ is the perturbed transverse velocity, with $\frac{\tilde{v}}{v} \ll 1$. Linearizing Eq. (3.1) in this way, get:

$$
\frac{\partial \tilde{v}}{\partial t}+v \frac{\partial \tilde{v}}{\partial z}=\frac{I B}{m} \cos (\omega t)
$$

Letting $\xi$ denote transverse displacement, $\tilde{v}$ is given by the convective derivative $\tilde{v}=\frac{d \xi}{d t}$. Expanding the convective derivative and linearizing, get:

$$
\tilde{v}=\frac{\partial \xi}{\partial t}+v \frac{\partial \xi}{\partial z}
$$

Substituting Eq. (3.3) into Eq. (3.2), obtain:

$$
\frac{\partial^{2} \xi}{\partial t^{2}}+2 v \frac{\partial^{2} \xi}{\partial t \partial z}+v^{2} \frac{\partial^{2} \xi}{\partial z^{2}}=\frac{I B}{m} \cos (\omega t)
$$

The initial conditions at the cathode $z=0$ corresponding to the assumption that the arc is fixed at the cathode emission spot are: $\left.\xi(z, t)\right|_{z=0}=0$ and $\left.\frac{\partial \xi}{\partial z}\right|_{z=0}=0$. Since we seek a steady-state solution in time with initial conditions in $z$, Eq. (3.4) can be solved by Fourier transforming in time and Laplace transforming in space (along $z$ ). One gets:

$$
\xi(z, t)=\frac{I B}{m \omega^{2}}\left[\cos \left(\omega t-\frac{\omega z}{v}\right)-\cos (\omega t)-\frac{z \omega}{v} \sin \left(\omega t-\frac{\omega z}{v}\right)\right]
$$

The resulting arc shape is thus a growing sinusoid with a wavenumber $k=\omega / v$. 
For $\frac{\omega z}{v} \ll 1$ (low frequency limit), sin and cos can be expanded in power series, giving:

$$
\xi(z, t)=\frac{I B \cos (\omega t)}{2 m v^{2}} z^{2}+\frac{I B \omega \sin (\omega t)}{3 m v^{3}} z^{3}+\mathcal{O}\left(\frac{z \omega}{v}\right)^{4}
$$

which gives parabolic deflection plus a cubic correction that is important for zero crossings of the magnetic field (when phase $\omega t$ is an odd multiple of $\pi / 2$ ). For DC magnetic field, in the limit $\omega \rightarrow 0$, Eq. (3.6) reduces to:

$$
\xi(z, t)=\frac{I B}{2 m v^{2}} z^{2}
$$

Parabolic deflection is thus recovered in the low frequency limit, as expected from the experiments described in Section 3.2.2.

Eq. (3.7) is consistent with the expression for DC deflection given in [3]:

$$
y=\frac{J B z^{2}}{2 \rho v^{2}}
$$

where $J$ and $\rho$ are current and mass densities, respectively. Assuming $J$ and $\rho$ to be average values over the arc cross-section and multiplying top and bottom of Eq. (3.8) by the cross-sectional area of the arc, one arrives at Eq. (3.7).

The above equations can be rewritten in dimensionless form by defining a length scale by $\frac{v}{\omega}$, equal to the distance the jet travels in an oscillation of the applied field, divided by $2 \pi$; the time scale by $\frac{1}{\omega}$, equal the oscillation period divided by $2 \pi$. Eq. (3.5) can then be rewritten as:

$$
\chi(\zeta, \tau)=M[\cos (\tau-\zeta)-\cos (\tau)-\zeta \sin (\tau-\zeta)]
$$

where $\chi \equiv \frac{\xi \omega}{v}, \tau \equiv \omega t, M \equiv \frac{I B}{m \omega v}$ and $\zeta \equiv \frac{z \omega}{v}$. The dimensionless number $M$, rewritten as $M=\frac{I B / \omega}{m v}$ can be interpreted as the ratio of transverse momentum imparted by the $\vec{I} \times \vec{B}$ force on a section of the arc in a time $\frac{1}{\omega}$ to the axial momentum of the jet. Eq. (3.9) is plotted in Figure 3.4 for two values of $\tau$. Figure 3.5 zooms in on region $\zeta \ll 1$, which represents low frequency, high jet speed, or short distances from the cathode cases. In Figure 3.6, $\chi(\zeta, \tau)$ is plotted for many values of $\tau$, 


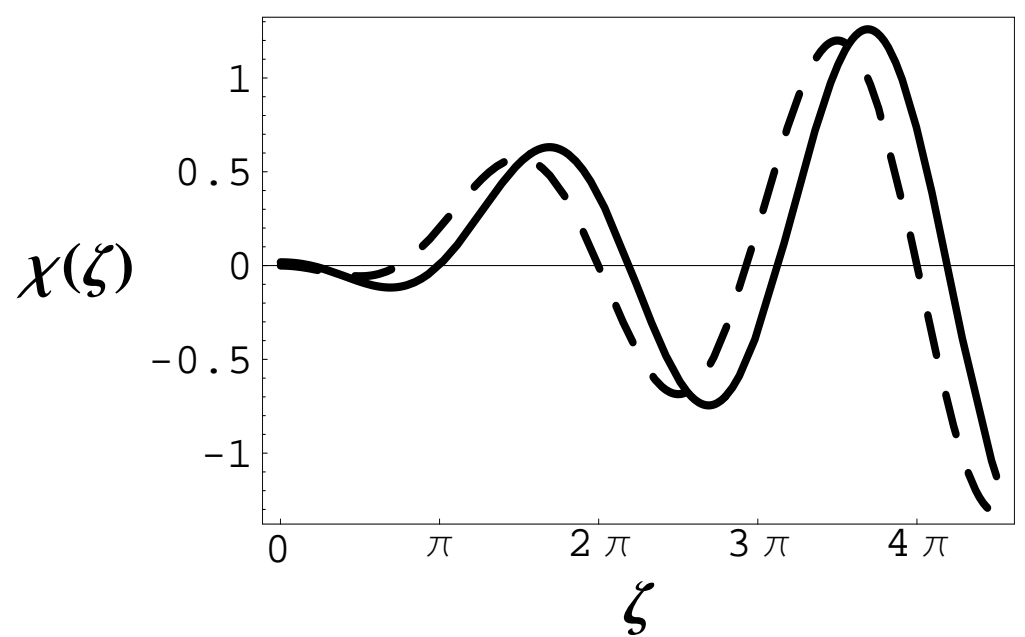

Figure 3.4: Derived arc shape and its time evolution: dashed curve shows arc shape at a particular time $\tau_{1}$, solid - a short time $\Delta \tau$ later. In this example, $\tau_{1}=0, \Delta \tau=0.6, M=-0.1$. Cathode is at $(0,0)$. See text for definition of dimensionless variables and numbers.

showing the expected envelope for arc displacement. The envelope can be defined as the locus of peaks of $\chi$, i. e. $(\zeta, \chi)$ such that $\frac{\partial \chi}{\partial \zeta}=0$. Applying this condition to Eq. (3.9), one gets:

$$
\left.\chi\right|_{\frac{\partial \chi}{\partial \zeta}=0}= \pm M[\zeta-\sin \zeta]
$$

Or, for $\zeta \gg 1$

$$
\left.\chi\right|_{\frac{\partial \chi}{\partial \zeta}=0} \approx \pm M \zeta
$$

That is, the envelope for arc motion is approximately a cone. The above equations are shown in Figure 3.6 as grey and broken lines, respectively.

\subsubsection{Arc length variation}

Given the equation for arc shape, the length of the arc as a function of time can be obtained. Since the arc voltage is a function of the arc length, any time variation in the latter will show up as a fluctuation on the former, as discussed 


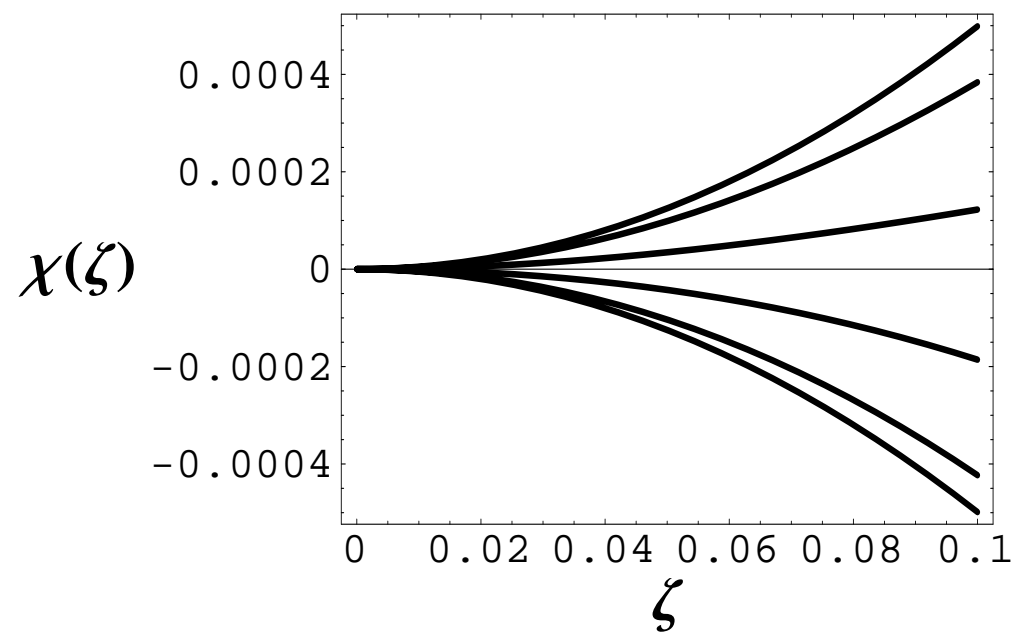

Figure 3.5: Arc shape for $\zeta \ll 1$ for several different times. $M=-0.1$. Cathode is at $(0,0)$.

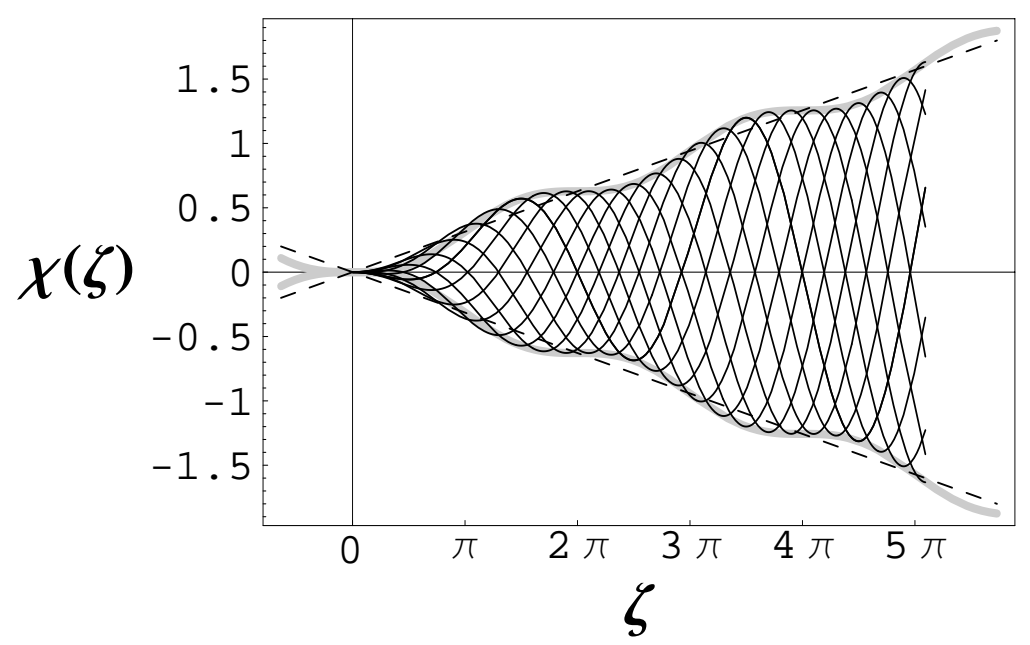

Figure 3.6: Overlay of arc shapes at different times shows the envelope of the arc displacement. The envelope for arc motion, given by $\pm M[\zeta-\sin (\zeta)]$, is shown in light grey. A cone given by lines $\pm M \zeta$ is shown dashed. Here, $M=-0.1$. Cathode is at $(0,0)$.

below in section 3.4.3. Using the dimensionless expression 3.9, arc length as a function of time is given by:

$$
L(\tau)=\int_{C(\tau)} d s
$$


where the line integral is along the arc column $C(\tau)$. Using $d s^{2}=d \zeta^{2}+d \chi^{2}$,

$$
L(\tau)=\int_{0}^{h} \sqrt{1+\left(\frac{\partial \chi}{\partial \zeta}\right)^{2}} d \zeta
$$

where $h$ is the distance between anode and cathode. From equation 3.9, $\frac{\partial \chi}{\partial \zeta}=$ $M \zeta \cos (\tau-\zeta)$. Therefore,

$$
L(\tau)=\int_{0}^{h} \sqrt{1+M^{2} \zeta^{2} \cos ^{2}(\tau-\zeta)} d \zeta
$$

Numerical evaluations of $L(t)$ for given values $h$ and $M$ show that $L(t) \propto \cos (2 \omega t)$, or, in other words, the arc length is varying at twice the applied magnetic field frequency $\omega$.

\subsubsection{Effect of cathode spot motion}

If the assumption of arc attachment spot being immobile on the cathode is relaxed, the arc shape, and therefore quantities inferred from it could change. The effect would depend on how much and how quickly the spot would move in response to the applied transverse field. Assuming the spot would move in the plane of motion of the arc column (transverse to the applied field), and that the surface of the cathode is flat and parallel to the anode surface, let the position of the spot on the cathode be given as $y=f(t)$. If the other assumptions made in the above derivation of arc shape (Section 3.3.1) are retained, the differential equation (3.4) for arc shape still holds. The only change is that the initial conditions at the cathode $z=0$ are now a function of time: $\left.\xi(z, t)\right|_{z=0}=f(t)$ and $\left.\frac{\partial \xi}{\partial z}\right|_{z=0}=0$. Incorporating these initial conditions in Laplace transform in $\mathrm{z}$, the solution, rewritten in the same dimensionless form as Eq. (3.9), becomes:

$$
\chi(\zeta, \tau)=\frac{\omega}{v}\left[f(\tau-\zeta)+\zeta f^{\prime}(\tau-\zeta)\right]+M[\cos (\tau-\zeta)-\cos (\tau)-\zeta \sin (\tau-\zeta)]
$$


If, furthermore, the spot is assumed to move harmonically and in phase with the driving field, with an amplitude a, $f(t)=a \cos (\omega t)$, arc shape becomes:

$$
\chi(\zeta, \tau)=\left(M+\frac{a \omega}{v}\right)[\cos (\tau-\zeta)-\cos (\tau)-\zeta \sin (\tau-\zeta)]+\frac{a \omega}{v} \cos (\tau)
$$

The shape of the arc in this case is thus identical to the anchored spot case, except for an increase in the amplitude of arc displacement $M \rightarrow M+\frac{a \omega}{v}$ and a harmonic motion of the column as a whole.

\subsubsection{Aerodynamic Drag}

In the above derivation the effect of the force of drag of the surrounding gas on the moving arc was neglected. The hydrodynamic Reynolds number for transverse motion of the arc is:

$$
R e=\frac{L v \rho}{\eta} \sim \frac{(0.01 \mathrm{~m})(1 \mathrm{~m} / \mathrm{s})\left(0.07 \mathrm{~kg} / \mathrm{m}^{3}\right)}{1.3 \times 10^{-4} \mathrm{~kg} / \mathrm{m} \cdot \mathrm{s}} \sim 5
$$

where $L=2 a$ is the arc diameter; $\rho$ and $\eta$ are mass density and viscosity of the surrounding gas, respectively, and their values are taken from [41]. Since Re is low, the aerodynamic drag per unit length of the column experienced by the arc as it moves through the surrounding gas is proportional to speed [43]:

$$
F_{\mathrm{drag}}=C \eta v
$$

where $C \sim 1$ is a dimensionless constant. In the above, the relevant $v$ is the component of velocity normal to the arc column, and, for $\frac{\partial \xi}{\partial z} \ll 1$ it is $\frac{\partial \xi}{\partial t}$. Adding this force term to the equation for arc shape, we get, in dimensionless form:

$$
\frac{\partial^{2} \chi}{\partial \tau^{2}}+2 \frac{\partial^{2} \chi}{\partial \tau \partial \zeta}+\frac{\partial^{2} \chi}{\partial \zeta^{2}}=M \cos (\tau)-G \frac{\partial \chi}{\partial \tau}
$$

where $G \equiv \frac{C \eta}{m \omega}$, the other quantities defined as before. Eq. (3.19) can be solved analytically using the same Fourier/Laplace method as before. The result shows that the effect of drag is small for $\zeta \sqrt{G} \ll 1$, i.e. for $z \ll v \sqrt{m / C \eta \omega} \sim 5 \mathrm{~cm}$ for the highest frequencies studied. For lower frequencies, the effect is negligible 


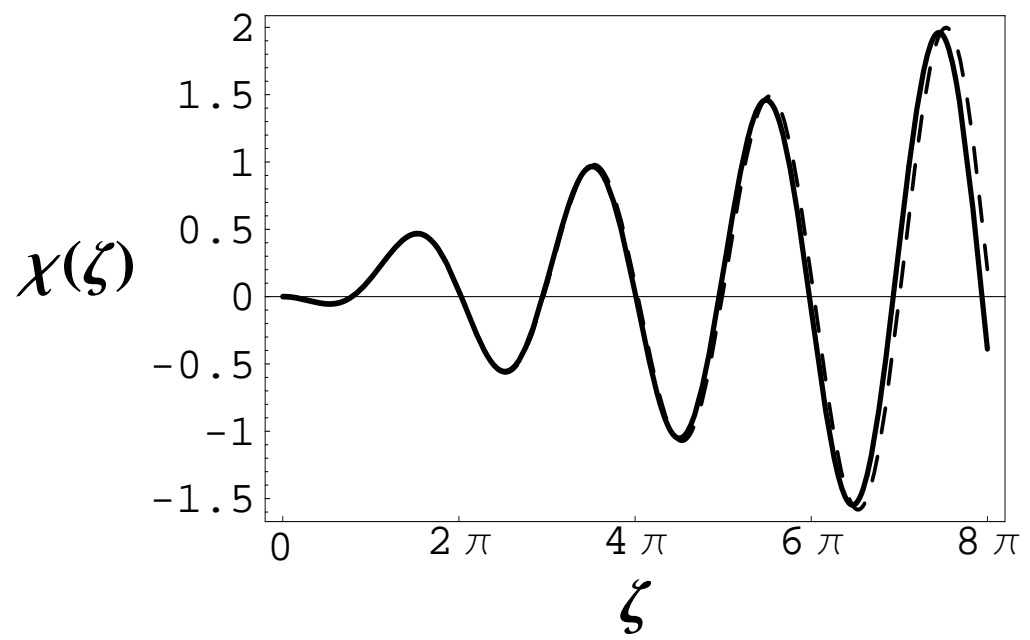

Figure 3.7: Arc shape with (solid line) and without (dashed) the aerodynamic drag effect. $M=-0.081, G=2.8 \times 10^{-3}$, frequency of applied magnetic field is $1212 \mathrm{~Hz}$. At this frequency, a $4 \mathrm{~cm}$ arc extends to $\zeta \approx 4 \pi$, and the drag effect is thus negligible.

even for longer arc lengths. Figure 3.7 shows an example of the arc shape with and without the aerodynamic drag effect for parameters corresponding to the high frequency experiments.

\subsubsection{Effect of self magnetic field}

In this section, an attempt is made to relax the assumption made until now of neglecting the self magnetic field effect in deriving the shape of the arc. Still following the 1-D approximation, the local effect of the self-field on a displaced arc column can be modeled as a force per unit length of the column dependent on the local curvature of the column and normal to it [44]:

$$
F_{\text {self }}=\frac{\mu_{0} I^{2}}{4 \pi R} \ln \frac{l}{a},
$$

where $I$ is the arc current, $R$ is local radius of curvature, $a$ is the radius of arc column, and $l$ is a characteristic length of the bending including geometrical factors. 
The force balance equation is then given by:

$$
m \frac{\partial \vec{v}}{\partial t}+m(\vec{v} \cdot \nabla) \vec{v}=\vec{I} \times \vec{B}+\vec{F}_{\text {self }}
$$

where $m$ is mass per unit length of arc, $I$ is arc current. Proceeding as in Section 3.3.2, get

$$
\frac{\partial^{2} \xi}{\partial t^{2}}+2 v \frac{\partial^{2} \xi}{\partial t \partial z}+v^{2} \frac{\partial^{2} \xi}{\partial z^{2}}=\frac{I B}{m} \cos (\omega t)+\frac{F_{\text {self }}}{m}
$$

For sufficiently small slope of the displaced arc column, the curvature can be approximated by the second derivative of the displacement [45]: $\frac{1}{R} \approx \frac{\partial^{2} \xi}{\partial z^{2}}$, where the minus sign is included to indicate the outward direction of the self-field force. Substituting expression (3.20) and making this approximation, the equation for arc displacement becomes:

$$
\frac{\partial^{2} \xi}{\partial t^{2}}+2 v \frac{\partial^{2} \xi}{\partial t \partial z}+\left[v^{2}+\frac{\mu_{0} I^{2}}{4 \pi m} \ln \frac{l}{a}\right] \frac{\partial^{2} \xi}{\partial z^{2}}=\frac{I B}{m} \cos (\omega t)
$$

Rewriting this in dimensionless form,

$$
\frac{\partial^{2} \chi}{\partial \tau^{2}}+2 \frac{\partial^{2} \chi}{\partial \tau \partial \zeta}+(1+N) \frac{\partial^{2} \chi}{\partial \zeta^{2}}=M \cos (\tau),
$$

where $N \equiv \frac{\mu_{0} I^{2}}{4 \pi m v^{2}} \ln \frac{l}{a}$, the other dimensionless variables and $M$ defined as before. The dimensionless quantity $N$ rewritten as $F_{\text {self }} /\left(\frac{m v^{2}}{R}\right)$ using Eq. (3.20), can be seen as the ratio of the self-field force to the centripetal force of the jet. Another interpretation of $N$ is the ratio of the magnetic energy to the kinetic energy in the jet: $\frac{\mu_{0} I^{2}}{4 \pi m v^{2}}=\left(\frac{B_{\text {self }}^{2}}{2 \mu_{0}} \pi a^{2}\right) /\left(\frac{m v^{2}}{2}\right)$, where $B_{\text {self }}=\frac{\mu_{0} I}{2 \pi a}$ is the self-field at arc radius $a$ and $\frac{B_{\text {self }}^{2}}{2 \mu_{0}}$ is the magnetic energy density. 
Since $N$ is dependent on $l$ only logarithmically, it is taken as a constant. Using the initial conditions and the method used in Section 3.3.2, the solution is:

$$
\begin{aligned}
\chi(\zeta, \tau)=M\left[\cos \left(\tau-\frac{\zeta}{1+K^{2}}\right) \cosh \left(\frac{K \zeta}{1+K^{2}}\right)-\right. \\
\left.\quad \frac{1}{K} \sin \left(\tau-\frac{\zeta}{1+K^{2}}\right) \sinh \left(\frac{K \zeta}{1+K^{2}}\right)-\cos (\tau)\right],
\end{aligned}
$$

where $K \equiv \sqrt{N}$.

In the limit $K \rightarrow 0$, Eq. (3.25), as expected, reduces to the arc shape with no self-field effect, given by Eq. (3.9). For sufficiently high applied magnetic field frequency when $K \zeta \gtrsim 1$, even if $K<1$, a significant effect is expected because of exponential growth of cosh and sinh. The arc shape given by Eq. (3.25) is plotted in Figure 3.8 for a value of $K$ consistent with the experimental arc parameters. The arc shape without including the self magnetic field effect is also shown for comparison. Clearly, inclusion of the self-field interaction in this manner dramatically affects the calculated arc shape for $\zeta>1 / K$. The envelope of arc displacement no longer grows linearly with distance from the cathode, as in Figure 3.6, but grows exponentially. The perturbation given to the arc shape by the oscillating applied field is then growing with a mechanism similar to the $m=1$ instability in a z-pinch [46].

In the low frequency and DC limit for which $\zeta \ll 1$, Eq. (3.25) can be expanded as:

$$
\chi=\frac{M}{1+K^{2}}\left[\frac{1}{2} \cos (\tau) \zeta^{2}+\frac{\sin (\tau)}{3\left(1+K^{2}\right)} \zeta^{3}\right]+\mathcal{O}\left(\zeta^{4}\right)
$$

The deflection is thus still parabolic to lowest order, with self-field contribution small for $K<1$. For $K \gtrsim 1$ however, the arc would be deflected less than expected without inclusion of self-field effect (compare to Eq. (3.6)).

Going back to dimensional quantities, the deflection in the DC $(\omega \rightarrow 0)$ limit becomes:

$$
\xi(z)=\frac{I B}{2 m v^{2}(1+N)} z^{2}
$$




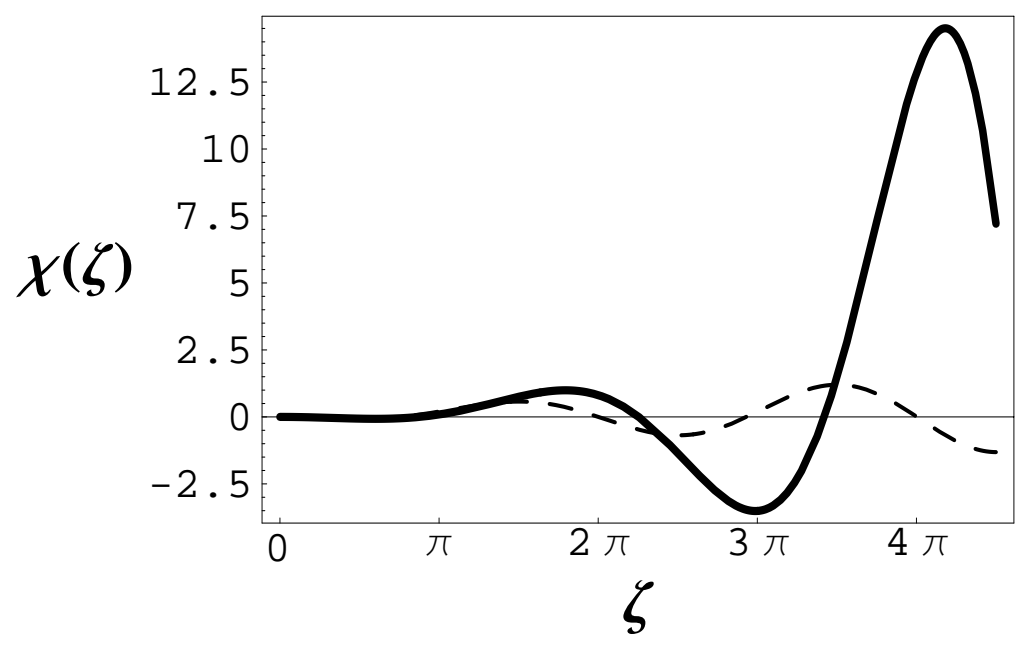

Figure 3.8: Calculated shape of the arc including the self magnetic field effect. $K=\sqrt{N}=0.44, M=-0.1$. For comparison, the shape calculated without the self-field effect $(K=0)$ is shown as the dashed curve. At $1200 \mathrm{~Hz}$, a $4 \mathrm{~cm}$ long arc extends to $\zeta \approx 4 \pi$.

where $N=K^{2}$ as before.

The numerical value of $N$ depends on the geometry of the deformed arc column. For parabolically deflected column, $N$ is given by (see the Appendix):

$$
N=\frac{\mu_{0} I^{2}}{4 \pi m v^{2}}\left(\ln \frac{h}{a}-1\right)
$$

where $h$ is the electrode separation, and $a$ is the arc radius. For non-parabolic (higher frequency) deflection, a numerical calculation discussed in the Appendix shows that Eq. (3.20) gives an overestimate for the self-field effect. The effect of self-fields in the experiment is discussed in Section 3.4.4.

\subsubsection{Non-uniform applied magnetic Field}

It has been assumed until now that the applied magnetic field is uniform over the length of the arc. If a gradient exists in the applied field, the driving term on the right side of Eq. (3.4) will be a function of $z$. Assuming the applied field 
decreases form a value $B$ at the cathode $(z=0)$ to a value $B_{a}$ at the anode $(z=h)$,

$$
B(z)=\frac{\left(B_{a}-B\right) z}{h}+B
$$

Substituting this $B(z)$ into the driving term on the right hand side of Eq. (3.4), and rewriting in dimensionless form, we obtain this modified equation for arc shape:

$$
\frac{\partial^{2} \chi}{\partial \tau^{2}}+2 \frac{\partial^{2} \chi}{\partial \tau \partial \zeta}+\frac{\partial^{2} \chi}{\partial \zeta^{2}}=M \cos (\tau)\left((b-1) \frac{\zeta v}{h \omega}+1\right),
$$

where $b \equiv B_{a} / B$. Using the same method as above, the solution is:

$$
\begin{aligned}
\chi(\zeta, \tau)=M[\cos (\tau-\zeta)-\cos (\tau)-\zeta \sin (\tau-\zeta)]+ \\
\quad \frac{M v(b-1)}{h \omega}[2 \sin (\tau)-2 \sin (\tau-\zeta)-\zeta \cos (\tau)-\zeta \cos (\tau-\zeta)],
\end{aligned}
$$

where the quantity in the first square brackets is the original solution for a uniform field. The ratio of magnitudes of the correction to the original solution is $(b-$ 1) $v / h \omega$. The correction is therefore small for $v / h \omega \ll 1$, or $\omega \gg v / h$, even if the magnetic field decreases to 0 at the anode $(b=0)$. In the present experiment, $v \sim 23 \mathrm{~m} / \mathrm{s}$ and $h \sim 0.04 \mathrm{~m}$. Thus, for frequencies greater than $\approx 100 \mathrm{~Hz}$, the correction due to an applied field gradient can be ignored.

For low frequencies, when $v / h \omega \gtrsim 1$, Eq. (3.31) can be expanded in powers of $\zeta$ as before, since in this limit $\zeta=z \omega / v \leq h \omega / v<1$ :

$$
\chi=\frac{M}{2} \cos (\tau) \zeta^{2}+M\left(\frac{1}{3} \sin (\tau)-\frac{(1-b) v \cos (\tau)}{6 h \omega}\right) \zeta^{3}+\mathcal{O}(\zeta)^{4}
$$

The largest difference in the arc shape due to non-uniformity would then occur at the anode, i. e. at $\zeta=h \omega / v$. The ratio of the third term to the first one would then equal $(1-b) / 3$. Thus the error in assuming a uniform applied field is $(1-b) / 3 \times 100 \%$. Measurements of the applied magnetic field in the present experiments show a maximum gradient of $20 \%$ over the $4 \mathrm{~cm}$ arc length, corresponding to $b=0.8$ and therefore the maximum error in neglecting the gradient is $7 \%$. 


\subsubsection{Range of validity of the model}

The validity of the derived model is limited by the assumptions listed in Section 3.3.1. The high collisionality assumption is justified since the collision frequency is greater than the cyclotron frequencies and the applied field frequency: $\tau_{e e} \sim \tau_{e n} \sim 10^{11} \mathrm{~Hz} . \tau_{c e} \sim 2.8 \times 10^{6} \mathrm{~B} \mathrm{~Hz} \sim 10^{8} \mathrm{~Hz}$ [39], and the highest applied field frequency is $2 \mathrm{kHz}$.

Assumption 3 is examined as follows. The arc current direction is always tangent to the arc column. Unit tangent vector to a curve $\vec{r}(t)=x(t) \hat{x}+y(t) \hat{y}+z(t) \hat{z}$ is given by $\vec{T}=\frac{\vec{r}^{\prime}(t)}{\left\|\vec{r}^{\prime}(t)\right\|}$. Parameterizing arc shape by distance from cathode z, get:

$$
\begin{gathered}
\vec{r}(z)=z \hat{z}+\xi(z) \hat{y} \\
\vec{r}^{\prime}(z)=\hat{z}+\frac{\partial \xi}{\partial z} \hat{y}
\end{gathered}
$$

From Eq. (3.5),

$$
\frac{\partial \xi}{\partial z}=\frac{M \omega z}{v} \cos \left(\omega t-\frac{\omega z}{v}\right)
$$

The direction of the arc current is then given by:

$$
\vec{T}=\frac{\hat{z}+\hat{y} \frac{M \omega z}{v} \cos \left(\omega t-\frac{\omega z}{v}\right)}{\sqrt{1+\left(\frac{M \omega z}{v}\right)^{2} \cos ^{2}\left(\omega t-\frac{\omega z}{v}\right)}}
$$

Therefore, transverse component of current is small for $\frac{M \omega z}{v} \ll 1$, i.e., $z \ll \frac{m v^{2}}{I B}$. From the experiment, $\frac{m v^{2}}{I B} \approx 4 \mathrm{~cm}$ at 5.6 Gauss and $150 \mathrm{~A}$. Thus, the assumption of small transverse current component is valid for distances from the cathode up to $4 \mathrm{~cm}$; further for smaller applied fields and higher arc currents (if $v \propto I$ as found in the present experiments - see Section 3.4.2, page 68).

The transverse component of velocity $\tilde{v}$ can be compared to the axial jet speed $v$ by evaluating $\tilde{v}$ using definition (3.3) and Eq. (3.5):

$$
\frac{\tilde{v}}{v}=M\left[\sin (\omega t)-\sin \left(\omega t-\frac{\omega z}{v}\right)\right]
$$


Thus, $\tilde{v}$ is small for $M \ll 1$, which is always the case in the present experiments $\left(M \leq 0.3\right.$ ). For $\omega \rightarrow 0$ (DC deflection case), Eq. (3.36) becomes $\frac{\tilde{v}}{v}=M \frac{\omega z}{v}$, or $\frac{\tilde{v}}{v}=\frac{I B z}{m v^{2}}$, and the condition for validity is then the same as derived above, namely $z \ll \frac{m v^{2}}{I B}$, or $z<4 \mathrm{~cm}$.

Assumption 4 can be verified by considering the displacement due to anisotropic heat flux in a curved arc column. This effect is discussed in [42], where the following expression is derived for the velocity of the arc temperature distribution:

$$
v_{T} \approx 10 d_{m}\left(\frac{\rho_{m}}{\rho_{e}}\right)\left(\frac{1}{R}\right)
$$

where $d_{m}$ is thermal diffusivity, $\rho$ is mass density, $R$ is the radius of curvature of the column, and subscripts $m$ and $e$ refer to values at the temperature maximum in the arc and externally to the arc, respectively. The direction of this motion is towards the center of curvature. In order estimate the importance of this effect, the displacement $\xi^{\prime}$ caused by $v_{T}$ over the transit time of the jet from the cathode to the anode can be compared to the displacement $\xi$ derived above neglecting this effect. From Eq. (3.9) the curvature is:

$$
\frac{1}{R}=\frac{\partial^{2} \xi}{\partial z^{2}}=\frac{\omega}{v} \frac{\partial^{2} \chi}{\partial \zeta^{2}}=\frac{\omega M}{v}[\cos (\tau-\zeta)+\zeta \sin (\tau-\zeta)] \sim \frac{\omega M}{v} \zeta
$$

$v_{T}$ therefore is:

$$
v_{T} \sim 10 d_{m}\left(\frac{\rho_{m}}{\rho_{e}}\right)\left(\frac{M z \omega^{2}}{v^{2}}\right)
$$

The displacement due to $v_{T}$ would then be:

$$
\xi^{\prime}=\int_{0}^{t} v_{T} d t=\int_{0}^{z} \frac{v_{T}}{v} d z=10 d_{m}\left(\frac{\rho_{m}}{\rho_{e}}\right)\left(\frac{M \omega^{2} z^{2}}{2 v^{3}}\right)
$$

Using the envelope of arc displacement Eq. (3.11), $\xi \sim M z$ and therefore:

$$
\frac{\xi^{\prime}}{\xi}=10 d_{m}\left(\frac{\rho_{m}}{\rho_{e}}\right)\left(\frac{\omega^{2} z}{2 v^{3}}\right)
$$


For this effect to be small, $\xi^{\prime} / \xi$ should be $\ll 1$. From Eq. (3.41), this implies:

$$
\begin{aligned}
z \ll\left(\frac{v^{3}}{\omega^{2}}\right) & \left(\frac{\rho_{e}}{5 \rho_{m} d_{m}}\right) \\
& \approx \frac{(23 \mathrm{~m} / \mathrm{s})^{3}\left(0.7 \mathrm{~kg} / \mathrm{m}^{3}\right)}{((2 \pi) 1200 \mathrm{~Hz})^{2}(5)\left(1.7 \times 10^{-2} \mathrm{~kg} / \mathrm{m}^{3}\right)\left(0.02 \mathrm{~m}^{2} / \mathrm{s}\right)}=0.09 \mathrm{~m}
\end{aligned}
$$

where density and diffusivity values are taken from [41]. Thus, the Assumption 4 is valid for $z \ll 9 \mathrm{~cm}$ for $f \leq 1200 \mathrm{~Hz}$ and $v \approx 20 \mathrm{~m} / \mathrm{s}$, which is the case for the present experiments. For frequencies lower than $1200 \mathrm{~Hz}$ and for higher jet speeds, the effect is negligible even for longer arc lengths.

\subsection{Application of Model to Experiment}

In this section, the above model is applied to the experimental results. The model for arc shape has two parameters that are not measured directly in the experiments: the axial jet speed $v$ and the linear mass density $m$. For DC and low frequency deflection, both of these parameters appear in a single coefficient (see Eq. (3.7)) that can be obtained from the parabolic fit but cannot be evaluated separately. For higher frequencies $\left(\frac{\omega z}{v} \gtrsim 1\right)$, the jet speed and mass density appear as independent parameters (Eq. (3.5)), and thus can be inferred from experimental data.

\subsubsection{Low Frequency Case}

Eq. (3.27) was used to obtain the quantity $\frac{I}{2 m v^{2}(1+N)}$ from low frequency deflection experiments. As discussed in Section 3.2.2 and shown in Figure 3.2, the low frequency response is found to be independent of the applied field frequency, as expected from Eq. (3.6). The deflection is parabolic and proportional to the applied field strength. 


\begin{tabular}{|c||cccc|ccccc|}
\hline Run \# & \multicolumn{4}{|c|}{ "Normal" Fit } & \multicolumn{5}{|c|}{ "Alternate" Fit } \\
$(1 / 30 / 98)$ & $\mathrm{v}(\mathrm{m} / \mathrm{s})$ & $\mathrm{M}$ & $\theta(\mathrm{rad})$ & $\mathrm{R}$ & $\mathrm{v}(\mathrm{m} / \mathrm{s})$ & $\mathrm{IB} / \mathrm{m \omega}(\mathrm{m} / \mathrm{s})$ & $\theta(\mathrm{rad})$ & $\mathrm{R}$ & $\mathrm{M}=\mathrm{IB} / \mathrm{m} \omega / \mathrm{v}$ \\
\hline $18 \_3$ & 23.70 & -0.0688 & 0.0085 & 0.946 & 23.70 & -1.63 & 0.0085 & 0.946 & -0.06878 \\
$17 \_25$ & 21.18 & -0.0873 & 0.0300 & 0.988 & 21.18 & -1.85 & 0.0300 & 0.988 & -0.08735 \\
$17 \_11$ & 22.79 & -0.0777 & 0.0066 & 0.943 & 22.79 & -1.77 & 0.0066 & 0.943 & -0.07767 \\
\hline
\end{tabular}

Table 3.1: Examples showing the fit results to be identical for two choices of fit parameters. The "normal" fit parameters are $v, M$, and $\theta$. The "alternate" fit parameters are $v, I B / m \omega$, and $\theta$. In the last column, the values of $M$ computed by dividing $I B / m \omega$ by $v$, which are obtained from the "alternate" fit are identical to the values of $M$ obtained from the "normal" fit.

\subsubsection{Higher Frequencies}

As mentioned in Section 3.2.1, the angle that the arc axis makes with the electrode axis varies slowly. In order to account for this offset, this angle has been added to Eq. (3.9) as a third fit parameter ${ }^{1}, \theta$ :

$$
\xi(z, t)=\frac{M v}{\omega}\left[\cos \left(\tau-\frac{\omega z}{v}\right)-\cos (\tau)-\frac{z \omega}{v} \sin \left(\tau-\frac{\omega z}{v}\right)\right]+\theta z
$$

Data points $(z, \xi(z))$ are obtained from the camera images. The phase of the applied magnetic field, $\tau=\omega t$ at each image taken by the camera is known precisely from the phase of the current in the coils. Eq. (3.43) is fitted to the data points with $M$, $v$, and angle $\theta$ as fit parameters using a non-linear least-squares curve fit [47].

The three fit parameters $M, v$, and $\theta$ are independent of each other. Even though $M=\frac{I B}{m v \omega}$ contains $v$, it also contains the linear mass density $m$, a quantity independent of the jet speed $v$ and the angle $\theta$. Fits using other choices of three fit parameters, such as $\frac{I B}{m \omega}, v$, and $\theta$ yielded identical curve fits and identical values of $v, m$, and $\theta$, as can be seen in the examples shown in Table 3.1.

Two example higher frequency fits to experimentally obtained arc shape are shown in Figure 3.9. Such fits are obtained for many arc shapes for a range of frequencies, arc lengths, and arc currents, with the goodness of fit similar to the

\footnotetext{
${ }^{1}$ Strictly speaking, this parameter is the slope of the arc axis w.r.t. the electrode axis, i.e. slope $=\tan (\theta)$, where $\theta$ is the angle between the two. However, since $\theta \ll 1, \tan (\theta) \approx \theta$.
} 

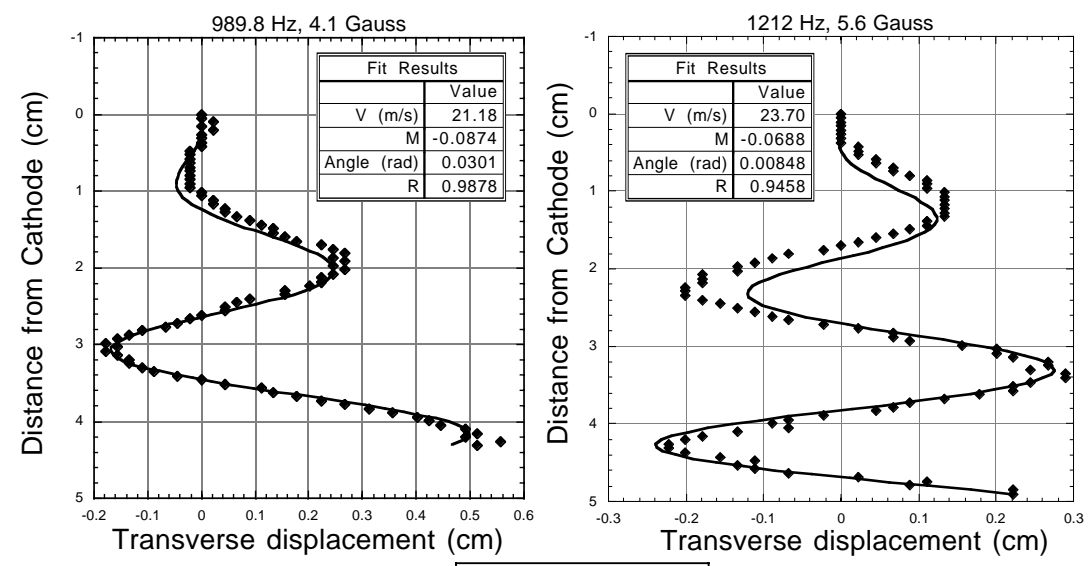

- Arc shape data

Curve fit

Figure 3.9: Examples of experimentally obtained arc shape fitted by the model (neglecting the self magnetic field effect). The jet speed $v$, interaction parameter $M$ containing the mass density, and the angle of the arc axis are the fit parameters. $\mathrm{R}$ is the fit correlation coefficient. Horizontal scales are enlarged. Arc current is $150 \mathrm{~A}$.

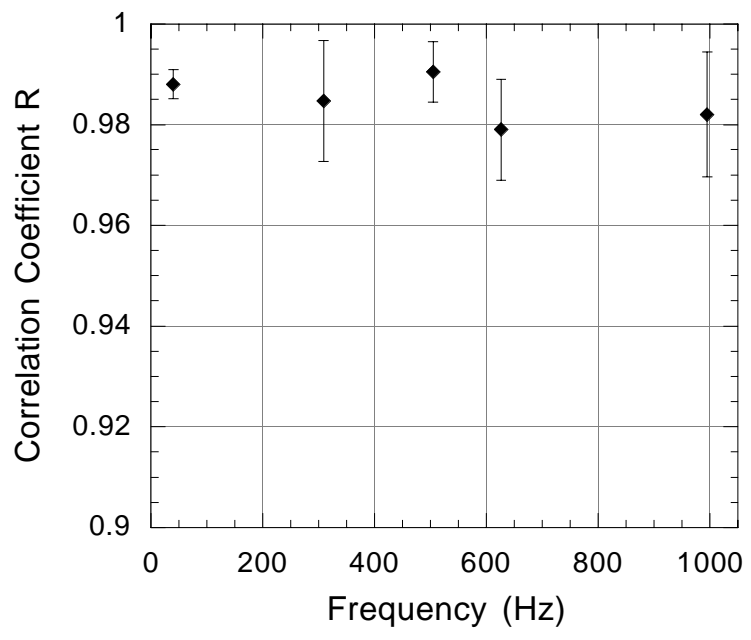

Figure 3.10: Correlation coefficient $\mathrm{R}$ of the model fit to the arc shape for several frequencies of applied magnetic field.

examples shown. The correlation coefficient $\mathrm{R}$ of the fits is plotted in Figure 3.10 for several frequencies, and is generally 0.95 or higher, and typically is 0.98 . 
The values of $v$ and $M$ can be determined uniquely from the fits. As can be seen in Eq. (3.43), the amplitude of the displacement of the arc column is proportional to the product of the dimensionless parameter $M$ and the jet speed $v$. However, Eq. (3.43) is very sensitive to the jet speed $v$, since for a fixed applied magnetic field frequency $\omega, v$ determines the wavelength of the displacement of the arc column. The nearly orthogonal roles of $v$ and $M$ in determining the wavelength and the amplitude of the fit, respectively, are illustrated in Figure 3.11. The figure shows two fits to the same arc shape: one for $v$ fixed at a value slightly above optimal, with $M$ as a fit parameter, the other — for $M$ fixed at a value slightly below optimal, with $v$ as a fit parameter, with the optimal values determined by fit with both $v$ and $M$ as fit parameters. The former shows a change in wavelength, while the latter - a change in amplitude. The sensitivity of the fit to variation in $v$ and $M$ is further illustrated in Figure 3.12, which shows the decrease in the goodness of fit as $v$ or $M$ is varied from their optimal values.

The jet speed $v$ inferred from the fits is found to be is independent of frequency and amplitude of the applied magnetic field to within the error bars, as can be seen in Figures 3.13 and 3.14. This is as expected, since the jet speed is determined by the constriction at the cathode and should not depend on the applied field [15]. Its value is significantly below Maecker's estimate of $\sim 200 \mathrm{~m} / \mathrm{s}$ [15]. Maecker's estimate is that of the maximum jet velocity near to the cathode region, while the jet velocity inferred from the fits is an average along the arc length and cross-section. Velocity profiles measured on similar arcs [48] show an initial rapid slowing down of axial flow within an arc diameter downstream of the cathode, due to momentum transfer to and entrainment of the surrounding gas [48], followed by much a smaller gradient further down the axis.

The inferred jet speed is also seen to be independent of the arc length as length was varied by $30 \%$ (Figure 3.15). This indicates that at approximately $3 \mathrm{~cm}$ from the cathode, the gradient in the cross-section averaged axial speed is smaller than the measurement error, i.e. $\frac{\partial v}{\partial z} \lesssim 5 \frac{\mathrm{m}}{\mathrm{s}} / \mathrm{cm}$.

The value of the jet speed inferred from the fits is in agreement with speeds of particles observed moving in the arc using a fast framing camera ${ }^{2}$ [49]. These

\footnotetext{
${ }^{2}$ Courtesy of Glen Wurden, Los Alamos National Lab.
} 


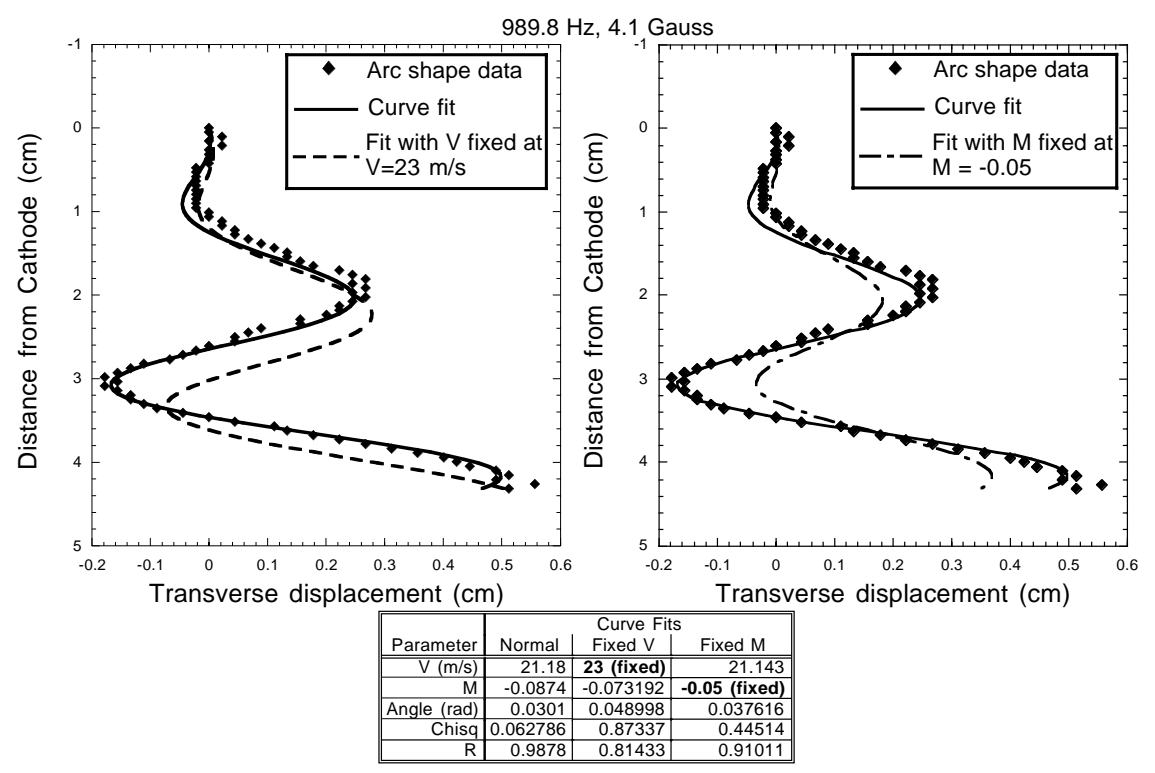

Figure 3.11: Fit parameters $v$ and $M$ are nearly orthogonal in determining the wavelength and amplitude, respectively, of the curve fit. On the left-hand side, the broken line is a fit with $v$ fixed at $23 \mathrm{~m} / \mathrm{s}$, slightly above the optimal $21.2 \mathrm{~m} / \mathrm{s}$, with $M$ as a fit parameter. As can be seen, the wavelength of the fit is longer than optimal. On the right-hand side, the broken line is a fit of the same arc shape data with $M$ fixed at -0.05 , below the optimal value of -0.087 , with $v$ as a fit parameter. In this case, the amplitude of the fit is smaller than optimal. Arc current is $150 \mathrm{~A}$; applied field frequency is $989.8 \mathrm{~Hz}$, amplitude is 4.1 Gauss peak.

particles apparently come off the graphite cathode. An example is shown in Figure 3.16. Particles were seen to traverse the length of the arc $(\approx 4 \mathrm{~cm})$ in $2 \mathrm{msec}$, giving a speed of $\approx 2,000 \mathrm{~cm} / \mathrm{sec}$. However, since the initial speed at which the particle is released from the cathode, and the amount of coupling of particle velocity to the gas flow has not been measured, it is not known how accurately the particle speeds represent the jet speed.

Having obtained the value of $v$ from the high frequency fits, one can now quantify the low frequency limit $\frac{z \omega}{v} \ll 1$ derived in Section 3.3.2. For a $4 \mathrm{~cm}$ long 150 A arc,

$$
\omega \ll \frac{v}{z} \sim \frac{23 \mathrm{~m} / \mathrm{s}}{0.04 \mathrm{~m}}=575 \mathrm{~s}^{-1}
$$



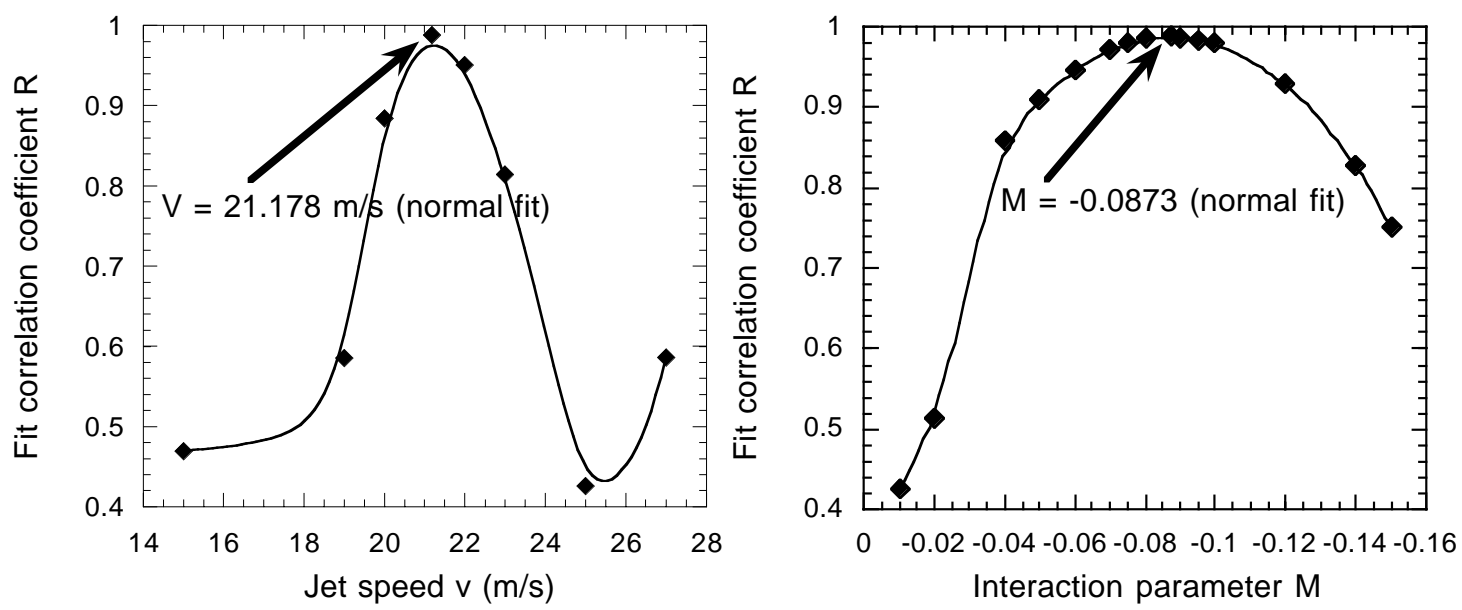

Figure 3.12: The goodness of fit decreases as the fit parameters $v$ and $M$ are varied from their optimal values. The left-hand side shows the fit correlation coefficient $\mathrm{R}$ for $M$ as a fit parameter with fixed values of $v$. The fit is very sensitive to $v$, with the optimal value at $v=21.2 \mathrm{~m} / \mathrm{s}$. The right-hand side gives $\mathrm{R}$ for $v$ as a fit parameter with fixed values of $M$. Although the fit is not as sensitive to $M$, the optimal fit is unique at $M=-0.087$. The arc shape data is the same as in Figure 3.11.

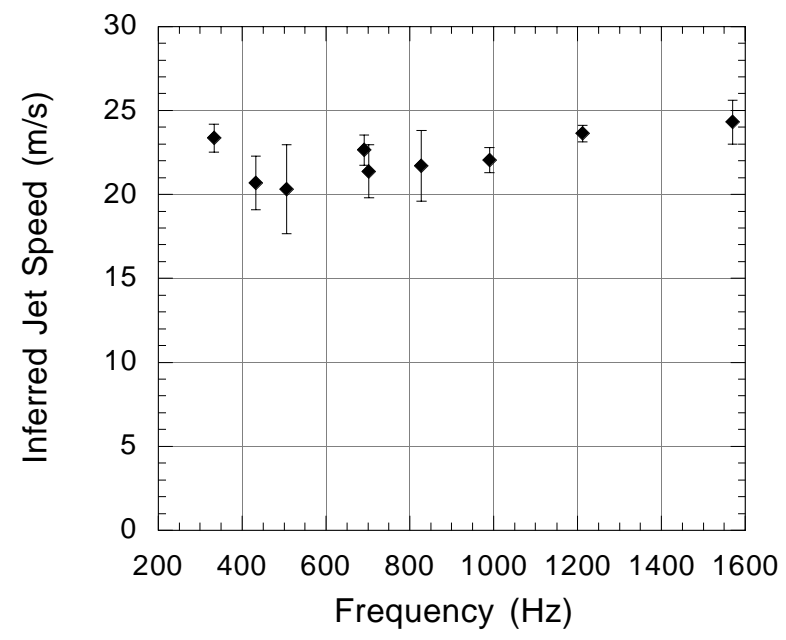

Figure 3.13: Inferred jet speed is independent of the frequency of the applied magnetic field. Arc current is 150 A. Error bars are one standard deviation from measurements at different phases of magnetic field. 


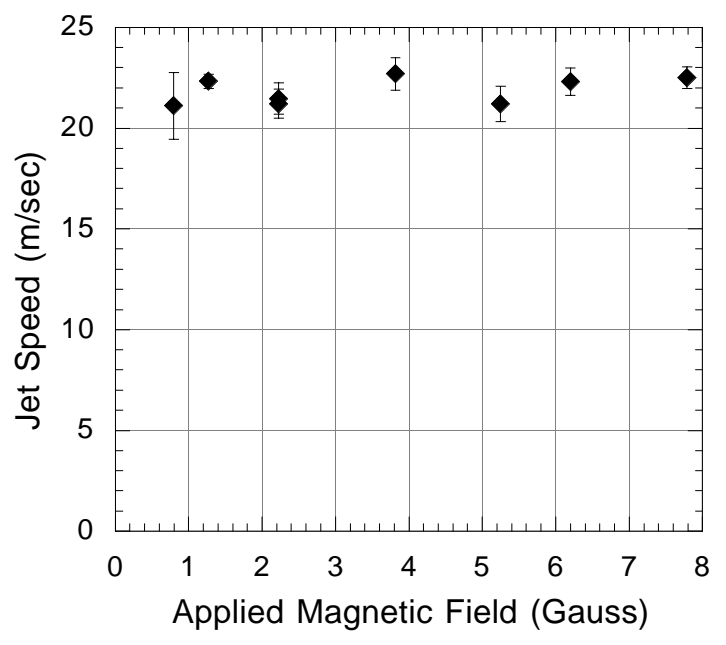

Figure 3.14: Inferred jet speed is independent of the applied field amplitude, as expected. Applied field frequency is $702.5 \mathrm{~Hz}$; arc current is $150 \mathrm{~A}$. Error bars are one standard deviation from measurements at different phases of magnetic field.

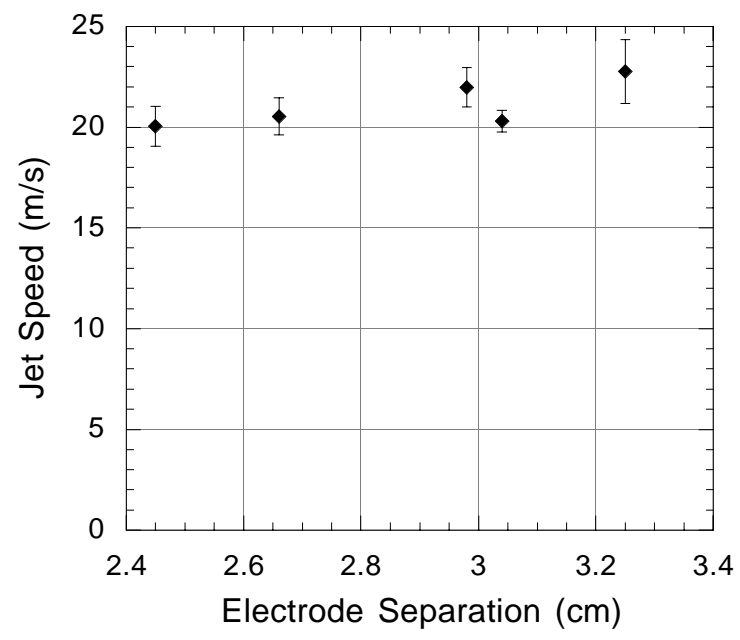

Figure 3.15: Inferred jet speed is independent of arc length. Arc current is $150 \mathrm{~A}$. Applied field frequency is $626 \mathrm{~Hz}$. Error bars are one standard deviation from measurements at different phases of magnetic field.

Thus, the low frequency limit, and therefore parabolic deflection, should be obtained for frequencies $f \lesssim 575 \mathrm{~s}^{-1} / 2 \pi \sim 90 \mathrm{~Hz}$. As mentioned in Section 3.2.2, the deflection is parabolic for frequencies up to $\approx 80 \mathrm{~Hz}$. 


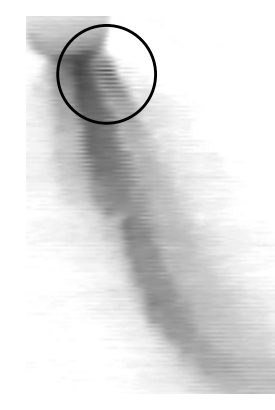

$0 \mathrm{~ms}$

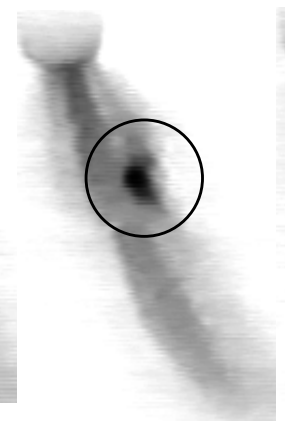

$1 \mathrm{~ms}$

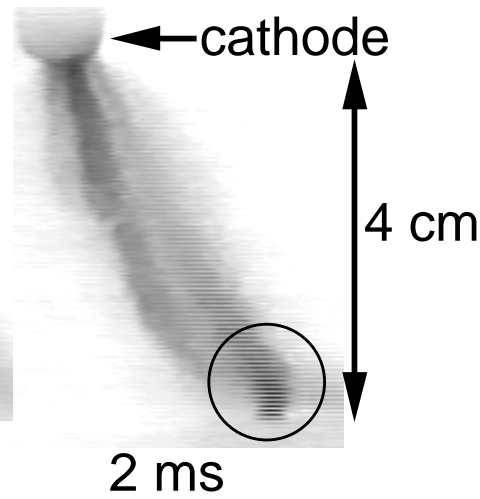

$2 \mathrm{~ms}$

Figure 3.16: Three consecutive frames showing the transit of a particle in the cathode jet. The camera framing rate was 1000 frames/sec. The particle (circled) comes off the cathode at $\mathrm{t}=0 \mathrm{~ms}$ and is seen to traverse the length of the arc $(\approx$ $4 \mathrm{~cm}$ ) in $2 \mathrm{msec}$, giving a speed of $\approx 2,000 \mathrm{~cm} / \mathrm{sec}$.

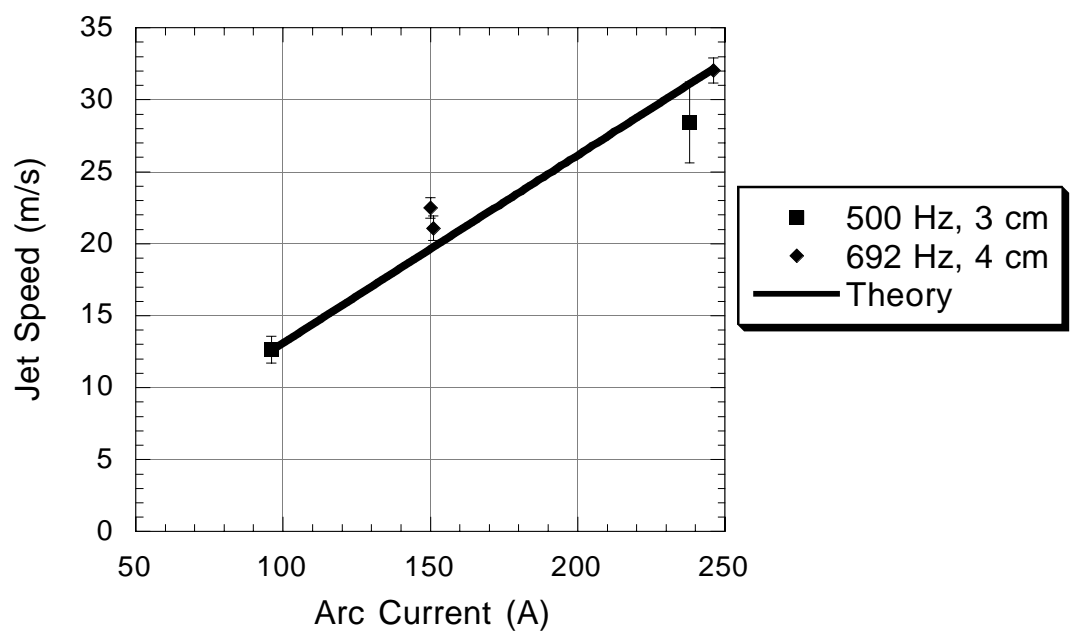

Figure 3.17: Inferred jet speed scales linearly with the arc current. Applied field frequency is $500 \mathrm{~Hz}$ and $692 \mathrm{~Hz}$ and electrode separation is $3 \mathrm{~cm}$ and $4 \mathrm{~cm}$, respectively. Error bars are one standard deviation from measurements at different phases of magnetic field.

Figure 3.17 shows the variation of inferred jet speed with arc current. The jet speed scales linearly in this current range. This is in agreement with Maecker's scaling of jet speed [15] $v=I \sqrt{\frac{\mu_{0}}{2 \pi^{2} r_{c}^{2} \rho}}$ if the cathode spot radius, $r_{c}$, remains constant. 


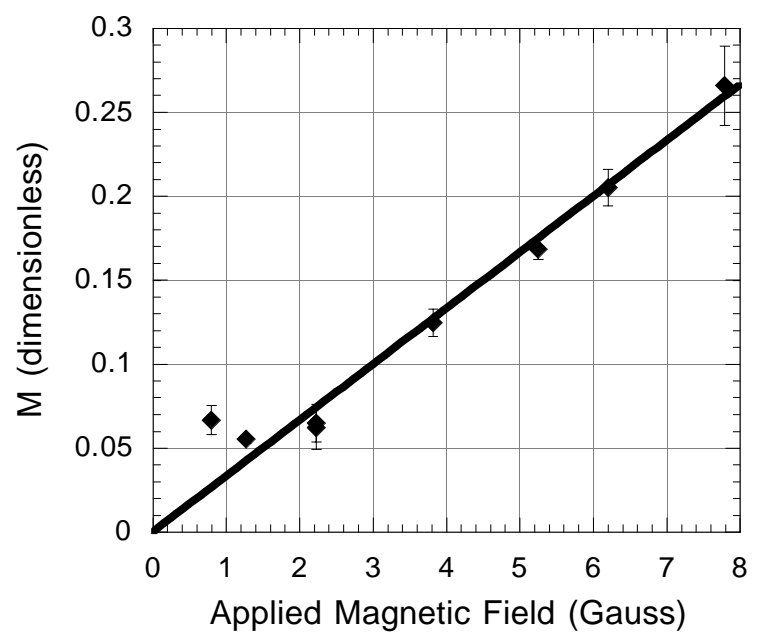

Figure 3.18: Arc response is linear in the applied magnetic field. The applied field frequency is $702.5 \mathrm{~Hz}$; electrode separation is $4 \mathrm{~cm}$; arc current is 150 A. Error bars are one standard deviation from measurements at different phases of magnetic field.

Figure 3.18 shows the scaling of the fit parameter $M$ with the applied field obtained from the high frequency fits. Since $M$ determines the amplitude of the deflection and the arc shape is well fitted by Eq. (3.43), it can be inferred that the deflection amplitude is proportional to the applied field in this range of applied field amplitudes, as expected.

The mass density inferred from the fits exhibits fluctuations from run-torun, which are not explained by the model, as can be seen in Figure 3.19. Based on measured temperature profiles of a $200 \mathrm{~A}$ carbon arc in air [9] and density values from [41], these linear mass density values imply an arc radius of approximately $0.7 \mathrm{~cm}$, corresponding to the $6,000-7,000 \mathrm{~K}$ isotherms. Large radial temperature (and therefore, density) gradients at this arc radius could account for the variation of this parameter.

The density values at $20 \mathrm{~Hz}$ were obtained by assuming a jet speed of $23 \mathrm{~m} / \mathrm{s}$ inferred from high frequency experiments and including the self-field effect as given by Eq. (3.27). It lies within the error bars of values at higher frequencies, indicating the model's consistency across this frequency range. Also shown is the value at $20 \mathrm{~Hz}$ if no self-magnetic field effect is included. 


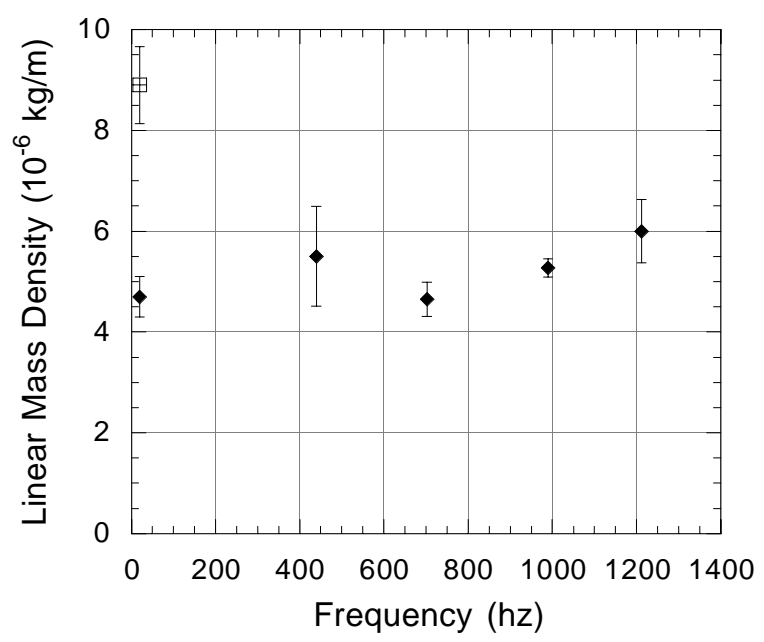

Figure 3.19: Inferred linear mass density vs. frequency of the applied magnetic field. Arc current is $150 \mathrm{~A}$. The average jet speed $v=23 \mathrm{~m} / \mathrm{s}$ inferred from the high frequency fits was used to get mass density from the parabolic fits of the low frequency $(20 \mathrm{~Hz})$ data point, including the self-magnetic field. Also shown (as the open square point) is the value at $20 \mathrm{~Hz}$ if no self-magnetic field effect is included. Error bars are one standard deviation from measurements at different phases of magnetic field.

\subsubsection{Arc voltage and current variation}

The voltage across the arc as well as the arc current exhibit fluctuations as a result of transverse magnetic field application. Since arc voltage is proportional to arc length, part of this fluctuation is due to variation in arc length as the shape of the arc evolves in time. On the basis of the model, as shown in section 3.3.3, arc length is found to vary at twice the applied field frequency. This harmonic is indeed present in the current and voltage waveforms, as can be seen from a segment of data waveforms in Figure 3.20. The presence of the fundamental frequency is not explained by the 1-D model, however. 


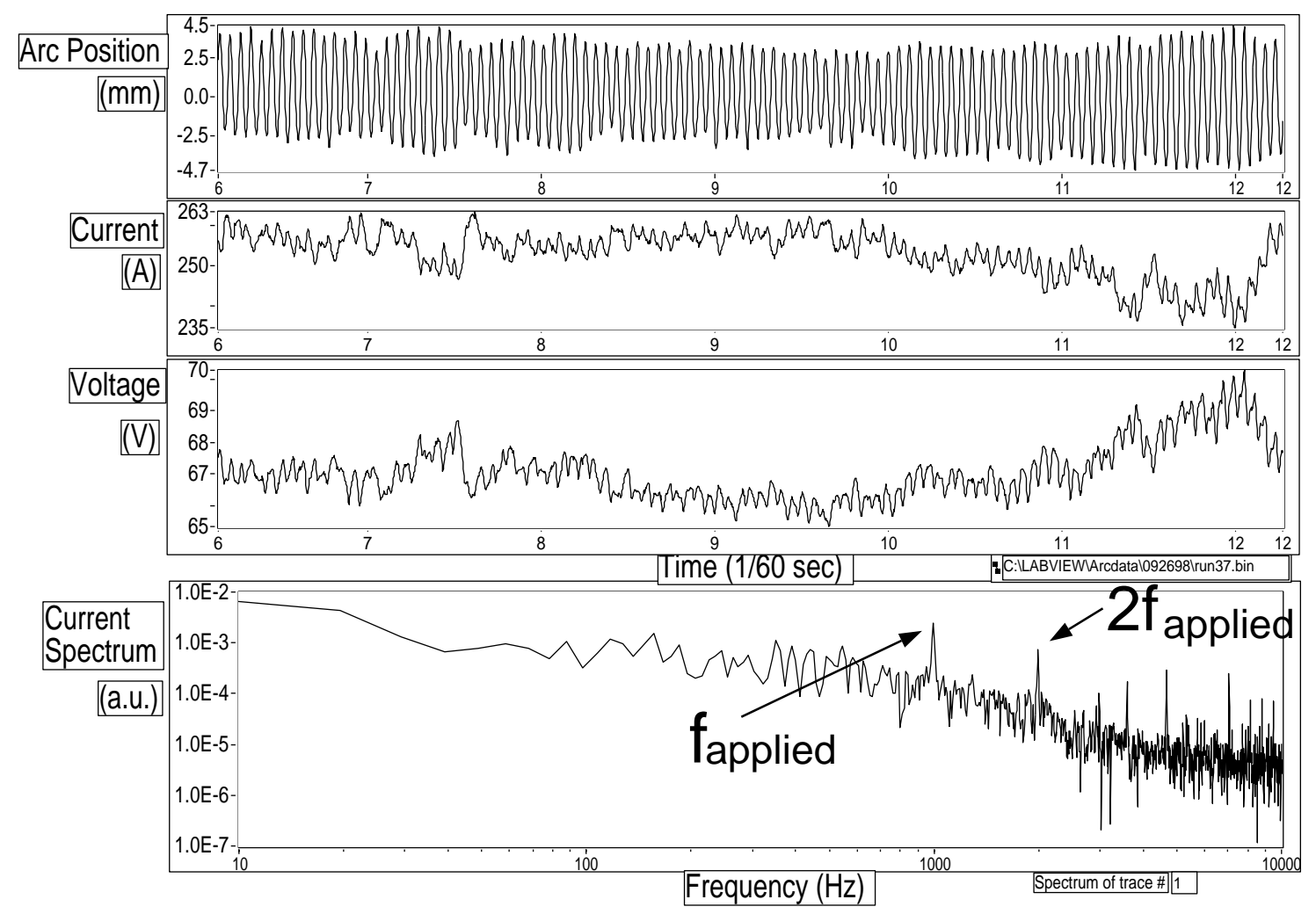

Figure 3.20: Waveforms acquired during application of transverse magnetic field. Applied frequency is $1 \mathrm{kHz}$. Arc position refers to the center of a cross-section of the arc at a fixed distance above the anode, and is moving at $1 \mathrm{kHz}$. A $2 \mathrm{kHz}$ harmonic is prominent on the current spectrum in addition to the fundamental. 


\subsubsection{Self-field effects}

In Section 3.3.6, an attempt was made to include the effect of the selfmagnetic field of the arc in the model by using an analytical approximation resulting in a simplified self-field term in Eq. (3.24). This approximation gives an exponentially growing envelope for the arc shape for high frequencies, as discussed in Section 3.3.6. In fact, the measured arc shapes do not show this exponential growth and the observed arc shape is best described by Eq. (3.5) without the simplified self-field term. This is illustrated in Figure 3.21, in which the fit produced using Eq. (3.25) with the simplified self-field term is compared with the fit without this term. As can be seen in the figure, the model with the self-field approximation is only able to reproduce the first half-wavelength on the measured arc shape, and rapidly diverges from the arc shape with further distance from the cathode. Thus, it is found that including the self-field approximation of Eq. (3.24) gives an arc shape inconsistent with the observations. Therefore, for all the high frequency results presented above, arc shape was fitted using Eq. (3.5) neglecting self-field effect.

Several possible reasons why Eq. (3.25) fails to model the arc shape correctly are as follows. First, the expression (3.20) for the force due to self magnetic field is a local approximation, neglecting the force due to the current in the rest of the arc column. Second, the expression is valid for $R \gg a$, where $a$ is the arc radius. For higher frequency $\mathrm{AC}$ experiments, however, $R \sim a$. Thus, in order to properly take the self-field into account, a 2D model is needed, which would include a current density $J(\vec{x})$ instead of total current. Third, the approximation

$\frac{1}{R} \approx \frac{\partial^{2} \xi}{\partial z^{2}}$ is only good for $\frac{\partial \xi}{\partial z} \ll 1$, whereas $\frac{\partial \xi}{\partial z}$ approaches 1 in the high frequency experiments. Finally, an effect balancing the self-field force could be present that is not accounted for in the model. The latter is unlikely since this effect would have to cancel the self-field effect over the whole frequency and amplitude range of the present experiment.

A better approximation (still in the thin-wire approximation) of the selfmagnetic field of the arc is calculated in Appendix A, in which the force due to self-field is integrated over the whole arc length. However, unlike the case of the simplified local analytical approximation discussed above, an analytical expression 


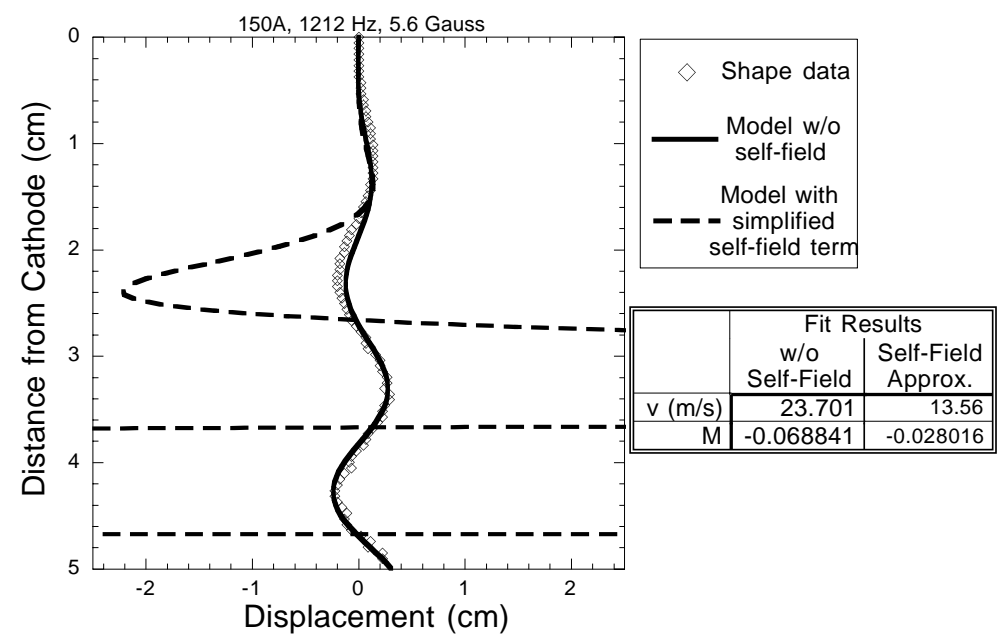

Figure 3.21: Experimentally obtained arc shape fitted by the models with and without the simplified self-magnetic field term, shown with dashed and solid curves, respectively. The jet speed $v$ and interaction parameter $M$ containing the mass density are the fit parameters. The model with the simplified self-field term gives exponential growth of displacement amplitude and can only approximate the measured arc shape for the first half-wavelength of the displacement.

for the arc shape cannot be obtained in this case. Therefore, no fits to measured arc shape were possible in this case. The computed arc shape did not deviate significantly from the arc shape without the self-field term (Figure A.1), indicating that when integrated over the whole arc length, the self-field effect is small. A fully two-dimensional numerical calculation including the current density profile would be needed to fully resolve the issue.

\subsection{An Industrial Application}

In this section, the model used for fitting the arc deflection in the present experiment is applied to an industrial scale DC arc furnace. Because this involves extrapolation of almost three orders of magnitude in arc current, only an order of magnitude estimate will be attempted.

The typical values for a modern DC steelmaking arc furnace are [2]: $I=$ $100 \mathrm{kA}, v_{\mathrm{jet}} \sim 1 \mathrm{~km} / \mathrm{s}$, and furnace diameter $=2 r \sim 10 \mathrm{~m}$. The size of the 
furnace and the arc current imply a magnetic field at the arc from the wire feeds of $B \approx \frac{\mu_{0} I}{2 \pi r}=40$ Gauss. Assuming an arc diameter of $10 \mathrm{~cm}$ and a mass density corresponding to air at $10,000 \mathrm{~K}$ of $1.7 \times 10^{-2} \mathrm{~kg} / \mathrm{m}^{3}$ gives linear mass density $m \sim 1 \times 10^{-4} \mathrm{~kg} / \mathrm{m}$. Using the model for DC arc deflection without the selfmagnetic field (Eq. (3.6)), $\xi=\frac{I B}{2 m v^{2}} z^{2}$, the deflection for $0.5 \mathrm{~m}$ long arc is $0.5 \mathrm{~m}$ at the anode, or $\approx 45^{\circ}$ from the axis. This is similar to the deflection observed in some industrial furnaces $[6,7]$, before any power cable re-routing to decrease the stray magnetic fields [5].

Eq. (3.27) can be used to include the self-magnetic field effect. Using the above values, $N \approx 6$. This gives a deflection of only $0.07 \mathrm{~m}$, or $8^{\circ}$ from the axis. Thus, the self-field effect might play a significant role in stiffening the arc.

This simplified estimate neglects complexities arising from the irregularities in the geometry of unmelted scrap anode, of current distribution in the melt and the electrode, the effect of an iron furnace shell on the magnetic fields, and the effects of submerging the arc in a layer of foamy slag as done in steelmaking. Some of these effects are discussed in Ref. 2,6,50.

\subsection{Discussion and Conclusions}

A systematic study was made of the behavior of $\approx 150$ A atmospheric pressure arc under the influence of DC and oscillating transverse magnetic fields. The experimental setup retains some of the features of industrial arc furnace and welding installations, i.e. the electrode geometry and materials, working gas composition and pressure, and the presence of transverse magnetic fields. The current range studied is similar to that utilized in welding.

A simple two-parameter model is proposed for the arc dynamics in transverse magnetic fields, based on a balance of $\vec{J} \times \vec{B}$ force and the inertia of the cathode jet. The model successfully describes the shape of the deflected arc for DC, low frequency, and high frequency magnetic fields, and gives reasonable values for the average jet speed $v$ and the linear mass density $m$. Both of these parameters are found to be independent of the applied magnetic field characteristics, as expected. 
The inferred average jet speed scales linearly with the arc current, as predicted by the Maecker's model, but is an order of magnitude below the estimated peak value.

The simplicity of the model is also its limitation. The two free parameters $v$ and $m$ it requires are determined empirically from the experiments. No prescription is given for relating these to actual cross-sectional and axial profiles of velocity and mass density. Resolution of these issues would most likely require a 2D model with radial and axial profiles of the arc, including pressure, mass density, velocity, and current density, such as computed in [51] together with a closed set of MHD equations.

The self-magnetic field effect on the arc shape have not been successfully incorporated into the analytical description of the arc deflection at higher frequencies. As discussed in the Appendix, the self-magnetic field analysis breaks down at higher frequencies and has not been iterated to achieve self-consistency. However, the consistency of the model without the self-field effect with the experiment for the whole frequency range studied indicates that self-field effects do not play a significant role in this parameter regime.

The ability to use the thin-wire approximation to describe the arc dynamics in magnetic fields indicates the possibility of using this model to analyze instabilities in high current arcs such as those described in [34]. Additionally, the importance of the cathode jet shown here indicates that it may play a role in determining stability properties as well.

In the above analysis, the average jet speed $v$ and linear mass density $m$ where determined from application of a high frequency transverse magnetic field. This method may be applicable as a diagnostic of flow speed and density in other arcs and plasma torches where strong flows are present.

The model of arc deflection presented here is directly applicable to the arc deflection in welding and also in material processing, where oscillating transverse magnetic fields have been used, for example, to spread the discharge over a wider processing area [52]. Although it involves a large extrapolation in arc current, the model gives a correct estimate for the deflection angle in steelmaking furnaces. 


\section{Chapter 4}

\section{Arc Instability}

The previous chapter dealt with driven regular motion of a stable arc under the influence of the applied magnetic field. The present chapter gives a description and analysis of unstable arc behavior observed under certain conditions with no external applied magnetic field. A discussion of the previous results on arc instability is given in Section 1.4.2.

The organization of this chapter is as follows: the experimental setup is described in Section 4.1, the experimental results are given in Section 4.2, a model for the instability is developed and applied to the experimental data in Section 4.3, cathode processes affecting arc stability are discussed in Section 4.4, an extrapolation to industrial scale arcs is considered in Section 4.5. Lastly, a discussion of the results and conclusions are given in Section 4.6.

\subsection{Experimental Setup}

The apparatus used in the instability study is the same as in Chapter 3 with the exception of the lack of the applied transverse magnetic field. The same type of instability has been observed with both a iron anode in the $\mathrm{MgO}$ crucible as well as with non-magnetic stainless steel anodes. However, all of the quantitative analysis presented below is for stainless steel anodes. Additionally, in order to determine 
the effect of cathode geometry on arc stability, cathodes of various tip diameters and tip shapes are utilized.

A typical "run" is done as follows. A particular kind of cathode to be studied is installed on the apparatus. The cathode is lowered to contact the anode. The current is raised to $150 \mathrm{~A}$. The cathode is raised approximately $1 \mathrm{~cm}$ from the anode, drawing an arc. Approximately $30 \mathrm{sec}$ is allowed before data taking for the anode surface to melt and the arc to steady. The experiments are carried out for 5-10 min, at variable currents and electrode gap, until the anode as a whole becomes too hot (side surface temperature $\approx 500^{\circ} \mathrm{C}$ ). If the arc extinguishes during the run, it is restarted by bringing the cathode back in contact with the anode and separating them as before. Each data acquisition sample consists of $0.5 \mathrm{sec}$ (30 fields) of image acquisition and $3.3 \mathrm{sec}$ fast data acquisition, synchronized to the camera gating. Typically, 20-30 such samples are collected per 5-10 min run.

\subsection{Experimental Results}

The instability occurs with no externally applied magnetic field. During the instability, the arc column develops a 3-dimensional wriggling helical shape, with the amplitude of the helix increasing toward the anode. The arc moves continuously on the anode surface. Cathode spot motion has also been observed during many unstable runs. A series of pictures of the arc taken using the gated camera during typical unstable behavior is shown in Figure 4.1. For comparison, views of a stable arc are also shown at the top.

\subsubsection{Overview of the instability}

The instability is accompanied by typically up to $\approx 10 \%$ RMS current fluctuations and $\approx 5 \%$ RMS voltage fluctuations. A plot of the current fluctuation amplitude in the (electrode gap vs. arc current) parameter space for a set of runs is shown in Figure 4.2. As can be seen in the Figure, the fluctuation level increases with current and electrode gap. For the standard $3 / 8^{\prime \prime}$ cathode, no instability has 


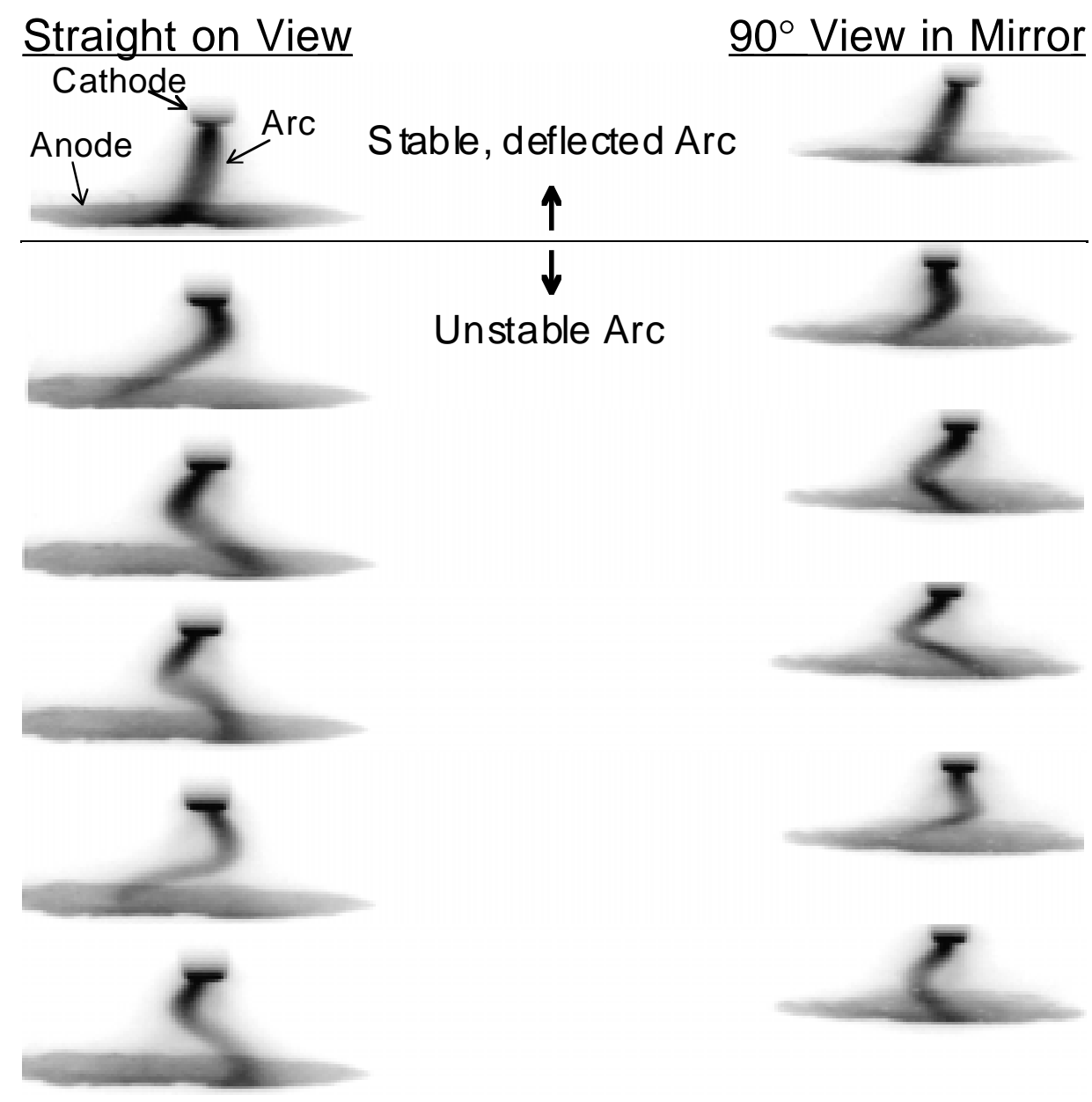

Figure 4.1: Sample images of unstable arc during low amplitude oscillation. Lefthand column shows direct view of the arc; right-hand column shows a $90^{\circ}$ view in the mirror. The horizontal scale is expanded 2.4:1 in direct view and 2.3:1 in the mirror view. The electrode gap is $3.1 \mathrm{~cm}$. The five unstable arc frames were picked from a 30 frame data sample to illustrate different arc column shapes.

been observed below 120 A. Also, although the amplitude of the voltage and current oscillation for unstable arc increases with electrode gap, the electrode gap, and therefore the arc length does not affect the threshold of the instability.

The arc instability has been found at all arc lengths in the range $1-7 \mathrm{~cm}$. For short arcs in the range of $1-2 \mathrm{~cm}$, especially when the fluctuation frequency is low, the arc column is deformed by only a fraction of a wavelength, and its motion as a whole along the cathode surface is apparent. An example of this is shown 


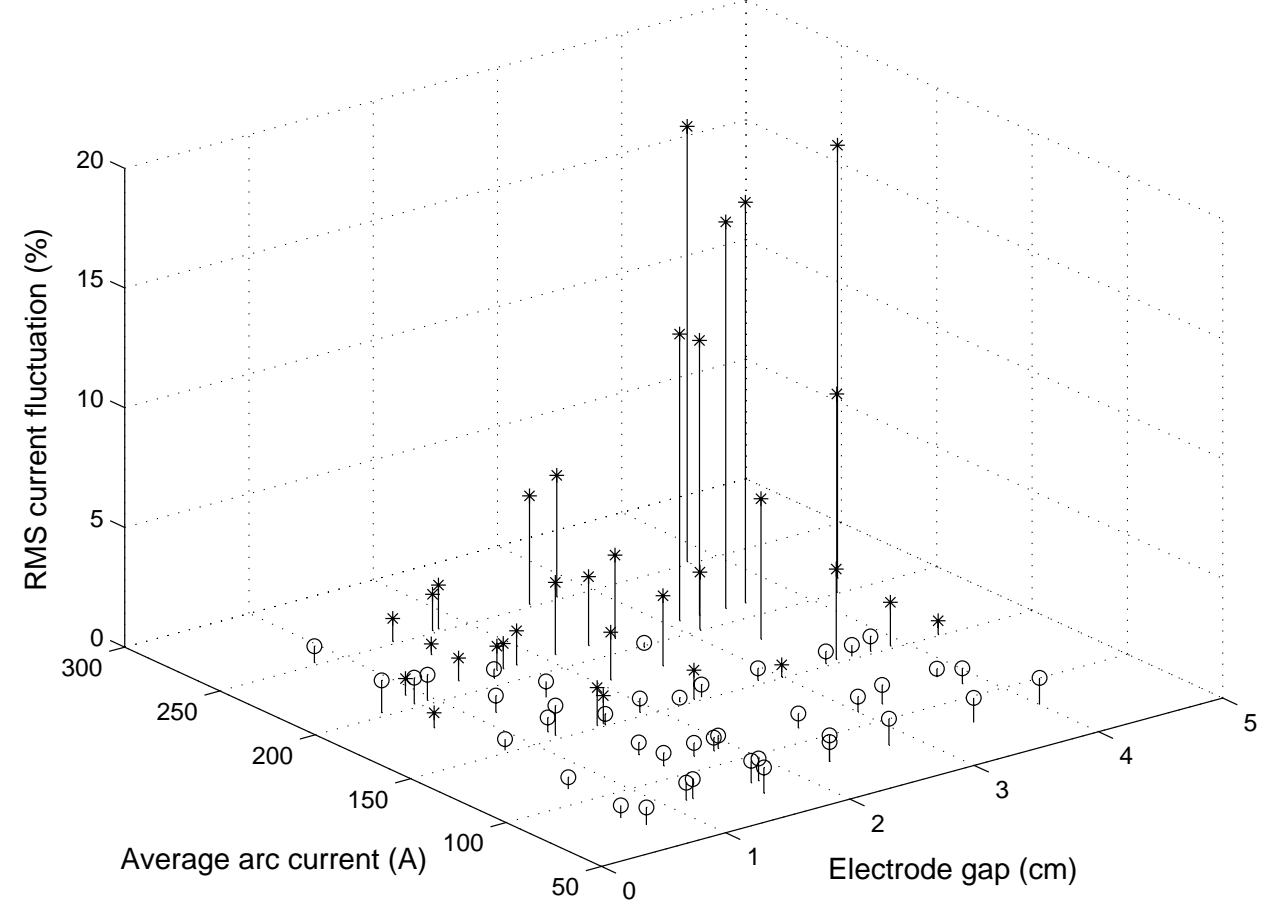

Figure 4.2: Measured RMS current fluctuation vs. current and electrode gap for a standard 3/8" cathode. Stable runs are marked with "o", unstable with "*". The fluctuation level increases with current and electrode gap. For a stable arc, the fluctuation level is typically $<1 \%$.

in Figure 4.3. Below approximately $0.5 \mathrm{~cm}$, the proximity of electrodes and the interaction of anode vapors and the impinging cathode jet can produce erratic arc motion regardless of the presence of the instability.

The frequency range of the current and voltage fluctuations is approximately 200-1000 Hz. A whistling sound is heard during many of the instability runs, as the frequency range is in the audio frequencies. For low amplitudes, the arc current fluctuation spectrum typically consists of strong peaks at a fundamental and a harmonic, with higher harmonics present during some runs. Typical waveforms and spectrum for low amplitude instability are shown in Figure 4.4. The peak frequencies vary by approximately 10\% during a 3 sec data acquisition sample.

Another feature seen in Figure 4.4 is that the current and voltage fluctuations are $180^{\circ}$ out of phase with each other. The same was found for a stable arc in 


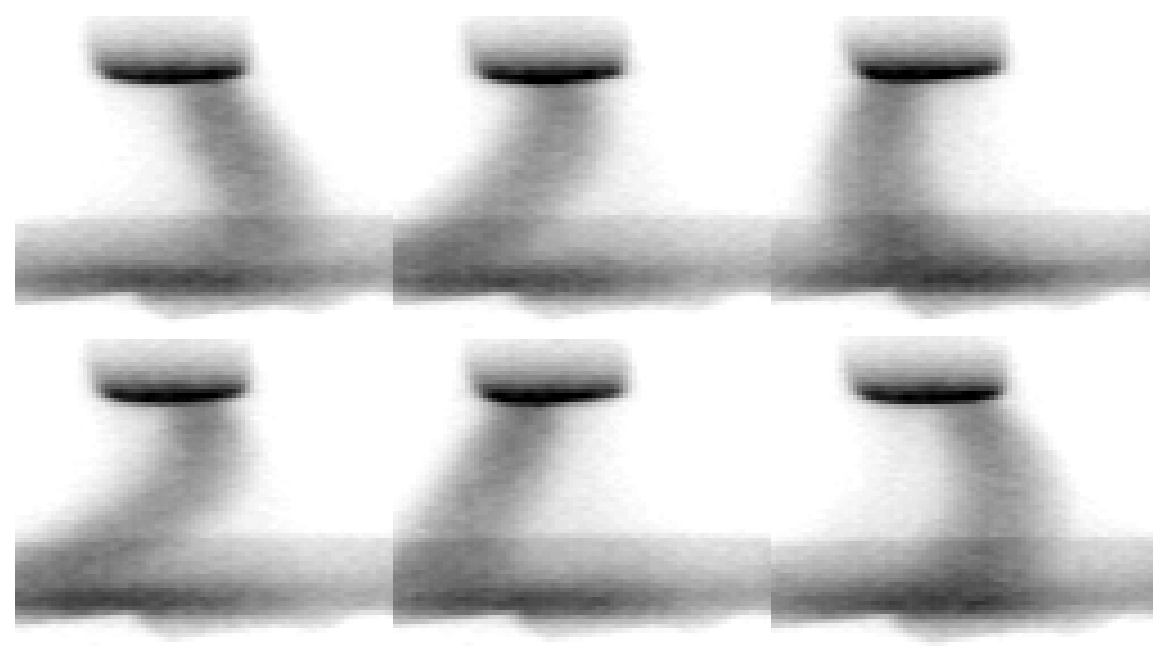

Figure 4.3: An example of instability at short electrode gap. Motion across the cathode surface is clearly visible. Electrode gap is $2.1 \mathrm{~cm}$; arc current is $240 \mathrm{~A}$; oscillation frequency is $\approx 300 \mathrm{~Hz}$. The horizontal scale is expanded 2.4:1.

applied AC transverse magnetic field (Chapter 3), and is discussed in more detail in Section 2.4 above.

The relative strength of the fundamental and its harmonic on the voltage and current can vary. In some instances, the oscillation on the current and voltage is dominated by the harmonic, with the fundamental showing up only weakly. The opposite case also occurs. Both can be seen in the example of Figure 4.5.

The frequency of arc column motion coincides with the fundamental frequency on the current and voltage waveforms. This was ascertained by inferring the column position along a horizontal line from the collimated photodiode array, described in Section 2.2.5. The inferred column position is also shown as a trace in Figures 4.4 and 4.5 .

The fundamental frequency of of current fluctuation for the same set of runs as in Figure 4.2 is plotted in Figure 4.6. Stable arc runs are indicated as zero frequency. The frequency of unstable runs is also plotted separately against the arc current in Figure 4.7 and against the electrode gap in Figure 4.8. No clear trend of frequency variation with the electrode gap can be seen. There is, however a possible trend of increasing frequency for higher current, which would be consistent 


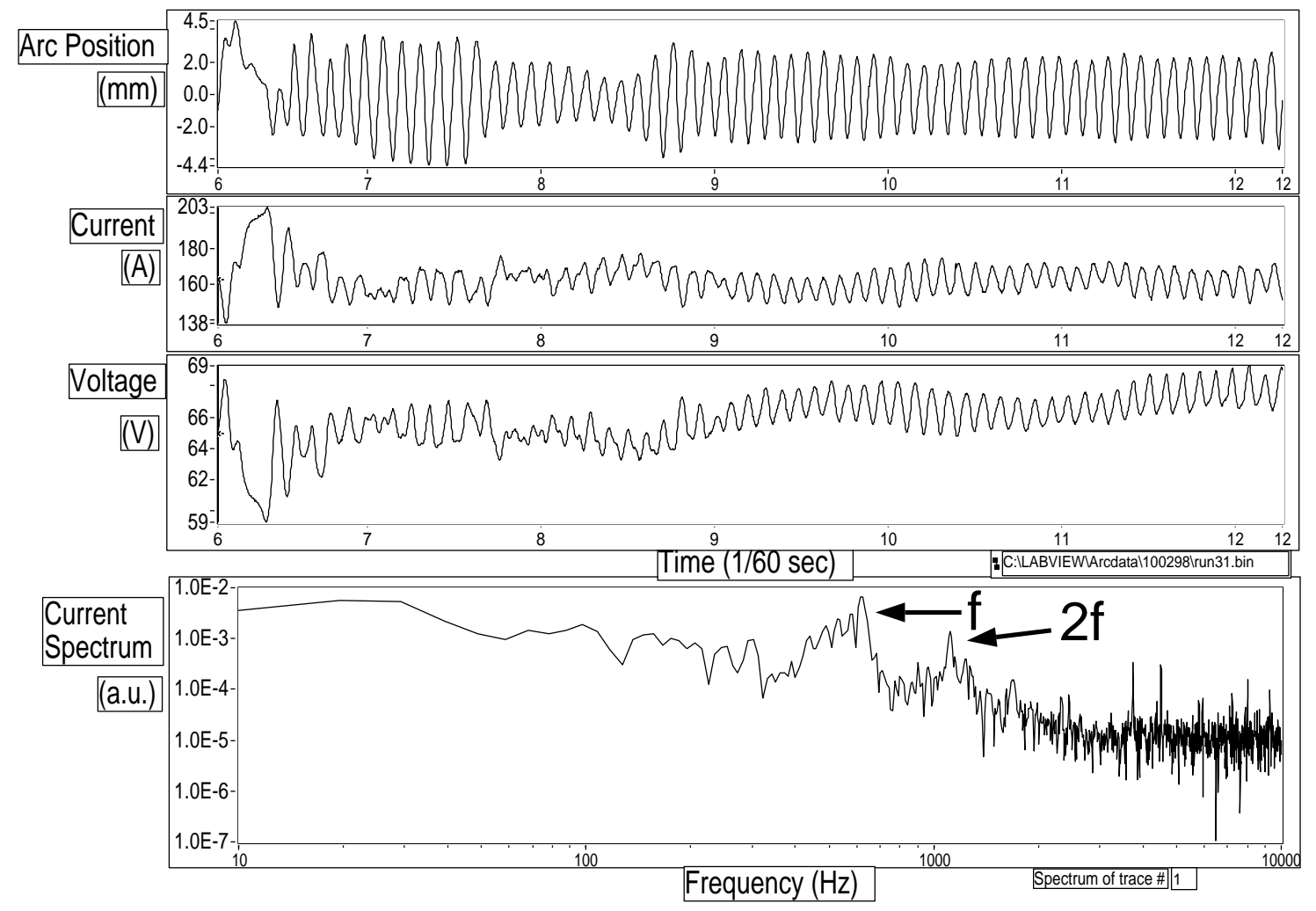

Figure 4.4: Waveforms of a typical low amplitude unstable run. Arc position refers to the center of a horizontal cross-section of the arc at a fixed distance above the anode, and is varying at $\approx 600 \mathrm{~Hz} . \mathrm{A} \approx 1200 \mathrm{~Hz}$ harmonic is prominent on the current spectrum in addition to the fundamental. In this and all the following waveform figures, the time scale is marked in fields taken by the gated camera at 59.939 fields per second, i.e., one unit on the time scale $\approx 1 / 60 \mathrm{sec}$. 0 on the time scale corresponds to the gate of the first field taken by the camera, 1 to the second, and so on. The current spectra are the amplitude of the Fast Fourier Transforms of the arc current over the time interval displayed.

with observations of Witkowski [33], which show the frequency increasing in the range 100-300 A and leveling off for 300-800 A. Large scatter in the data due to frequency variation over time and a limited current range preclude a more definite statement in the present experiments.

Instability onset can be gradual - it does not seem to require a finite "kick" for initiation. Figure 4.9 shows an example of such a gradual buildup of the oscillation, first in the column motion, then in the voltage and current waveforms. 


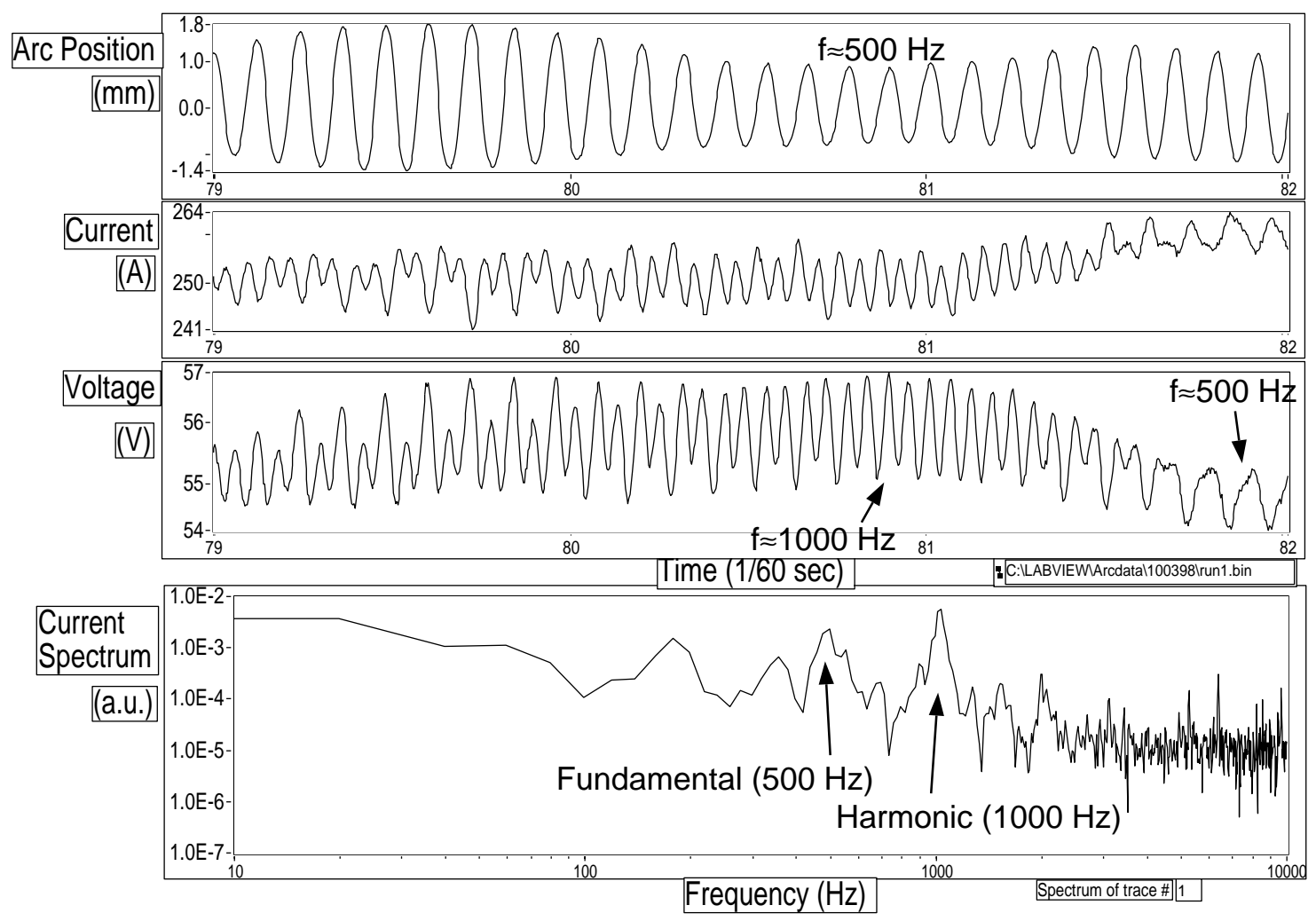

Figure 4.5: The relative strength of the fundamental and its harmonic on the voltage and current traces varies. In this example, the oscillation between 80.5 and 81.3 on the time scale is dominated by the harmonic, while between 81.5 and 82 the fundamental is prominent.

The presence of the instability of the column motion prior to the oscillation developing on the current also indicates that a current oscillation is not a part of the mechanism of the instability, at least at low amplitudes.

Instability at low amplitude can be intermittent, occurring in bursts that last from several to thousands of oscillation periods. Figure 4.10 shows the waveforms during such behavior. Moreover, even at higher currents (>180 A), the arc can remain stable for up to a minute or more after its initiation before the instability sets in. Once unstable bursts start to appear, the amplitude oscillation and the duration of the bursts typically increases over time, until the arc is wriggling continuously with such a large amplitude that it often leads to arc extinction. The time scale for this development is tens of seconds. 


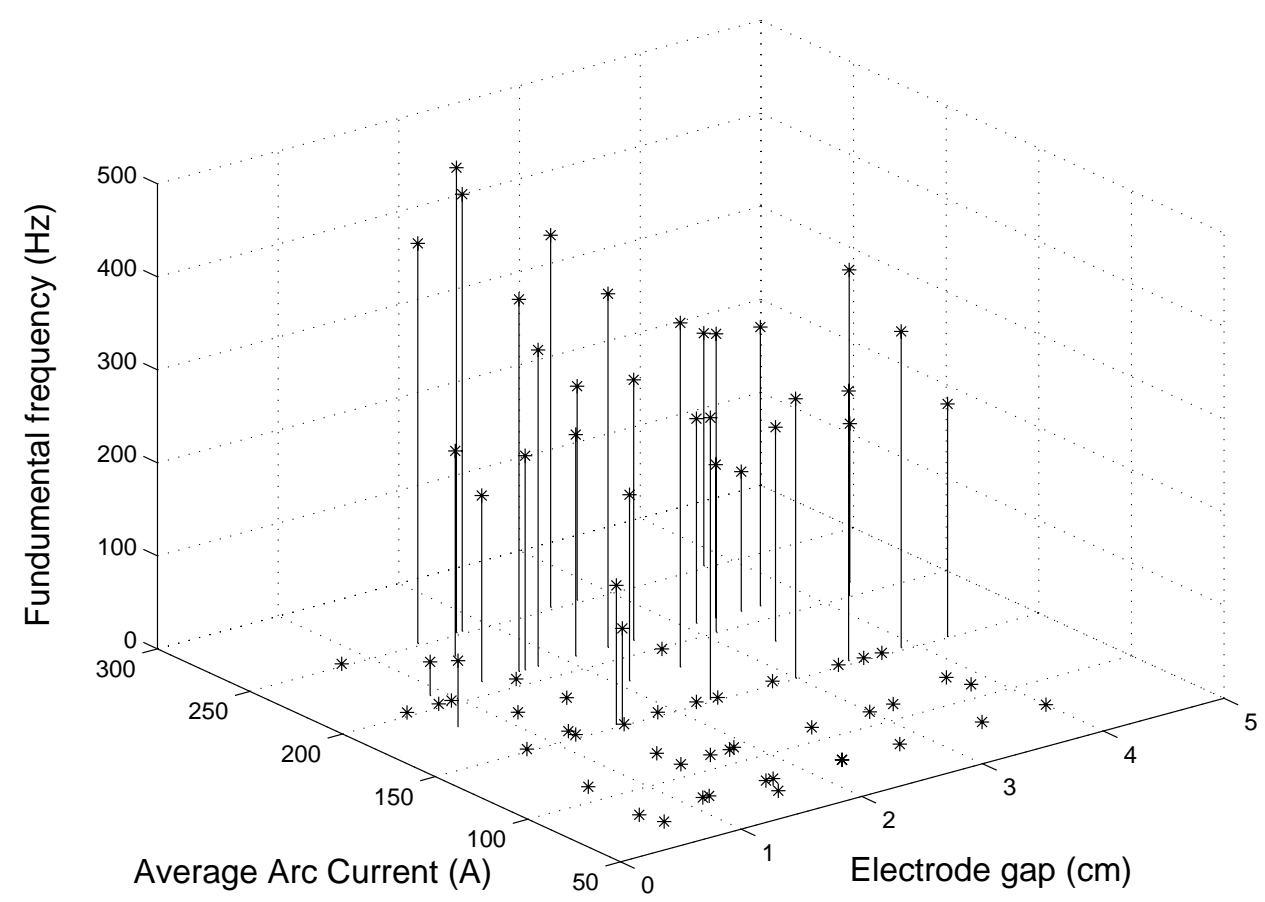

Figure 4.6: Measured fundamental frequency of current fluctuation vs. the arc current and interelectrode gap. Frequency is shown as 0 for stable runs. The frequency is taken as the fundamental peak in the amplitude spectrum of the current waveform over the $3.3 \mathrm{sec}$ data window. For large amplitude, only an approximate frequency in the middle of a range could be assigned.

For large amplitude instability, the spectrum no longer has distinguishable peaks, but is broad with a power law characteristic, as shown in Figure 4.11.

The shape of the arc column during instability is not always a three-dimensional helix: there are instances when the arc column is wriggling in a plane (see Figure 4.12). There are also instances when the spatial wavelengths in two planes of view are different.

Runs have been noted when the arc became stable after a long period of instability. In these cases and others it is found that by bringing the cathode back in contact or close proximity with the anode while the arc is on, then separating the two, instability can be restored. This is apparently due to a dependence on the cathode geometry, discussed below. 


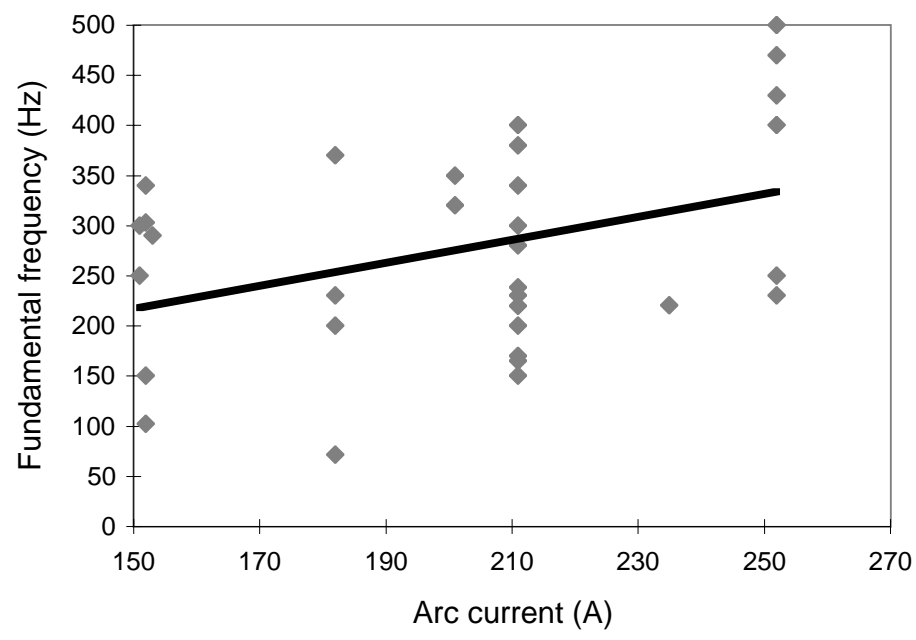

Figure 4.7: Measured fundamental frequency of current fluctuation vs. the arc current for the same set of runs as in Figure 4.6. A least-squares fit is also shown, indicating a possible trend of increasing frequency with current.

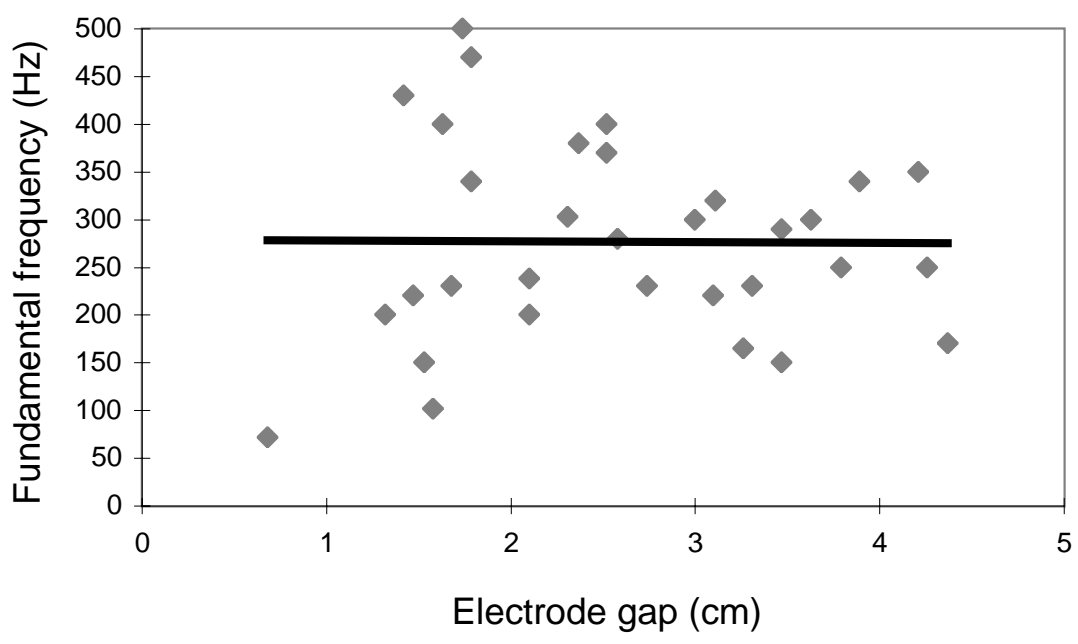

Figure 4.8: Measured fundamental frequency of current fluctuation vs. the electrode gap for the same set of runs as in Figure 4.6. A least-squares fit is also shown. The current range is $150-250 \mathrm{~A}$.

\subsubsection{Cathode geometry dependence}

It was found that the geometry of the cathode tip plays a crucial role in the arc stability. The geometries of the several classes of cathodes used in the stability experiments are shown in Figure 4.13. The dimensions labeled on the Figure as 


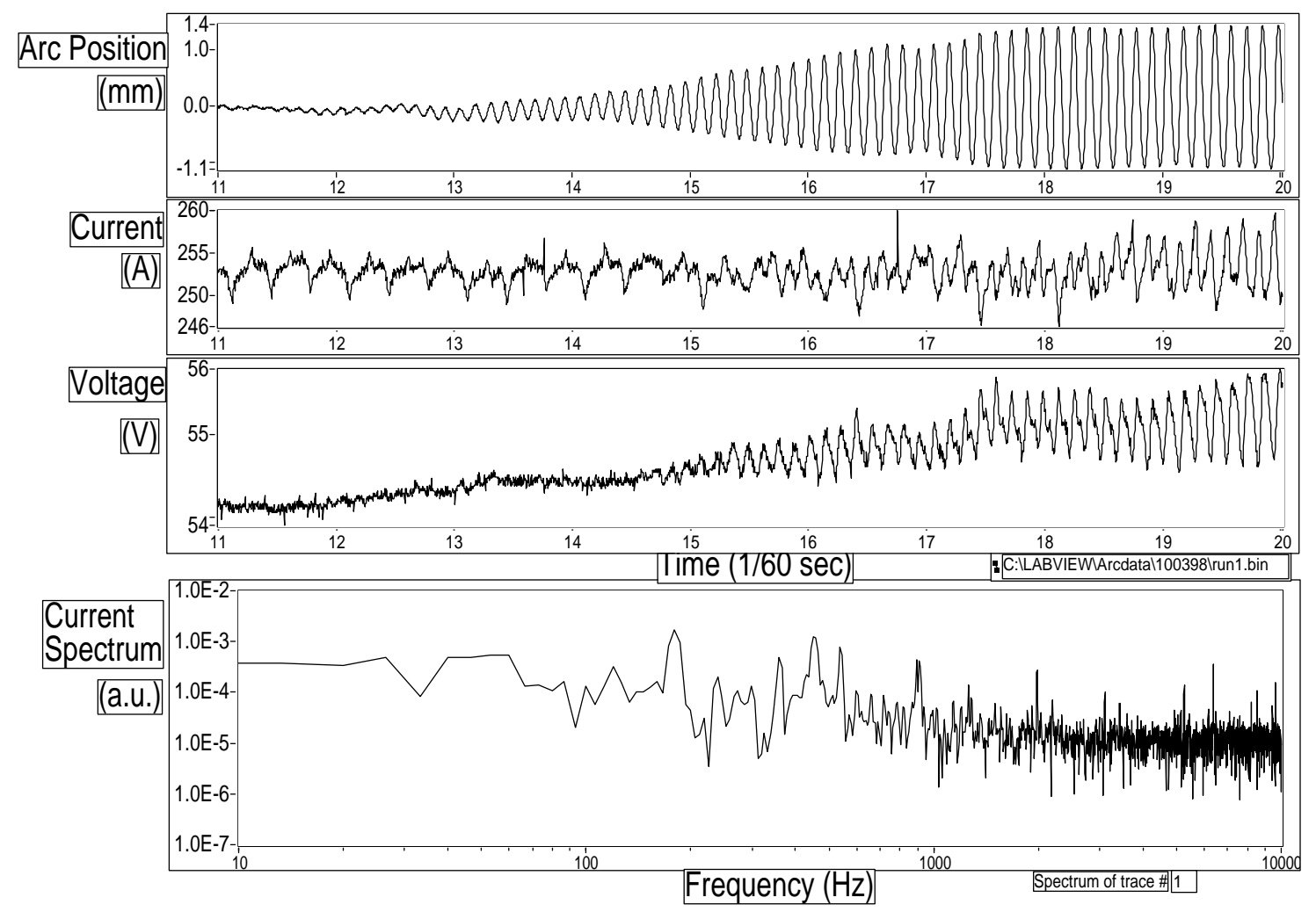

Figure 4.9: The onset of the instability can be gradual. The column begins oscillating ("Arc Position" trace) before the oscillation shows up on the voltage and current traces. The $180 \mathrm{~Hz}$ signal on the current trace noticeable prior to the instability onset is associated with the power supply SCR's.

"D", "L", etc. are given in Table 4.1 for each variation used. The Table also specifies whether the arc was stable or unstable with each particular cathode.

The stability data of Table 4.1 is summarized by a plot of occurrence of instability against the area of the cathode tip, shown in Figure 4.14. As can be seen in the Figure, for solid cylindrical cathode tips, the arc is unstable only in the range of $20-28 \mathrm{~mm}^{2}$ for the range of currents studied.

The cathode diameter dependence of the instability is dramatically demonstrated by the experiment with the "necked" cathode (\# 13 of Table 4.1). As can be seen in Figure 4.13(d), this cathode has two narrow "necks" machined in it with a diameter smaller than the lower stability threshold. The arc was unstable after approximately 15 sec of burning on the lower conical tip, stable while burning 


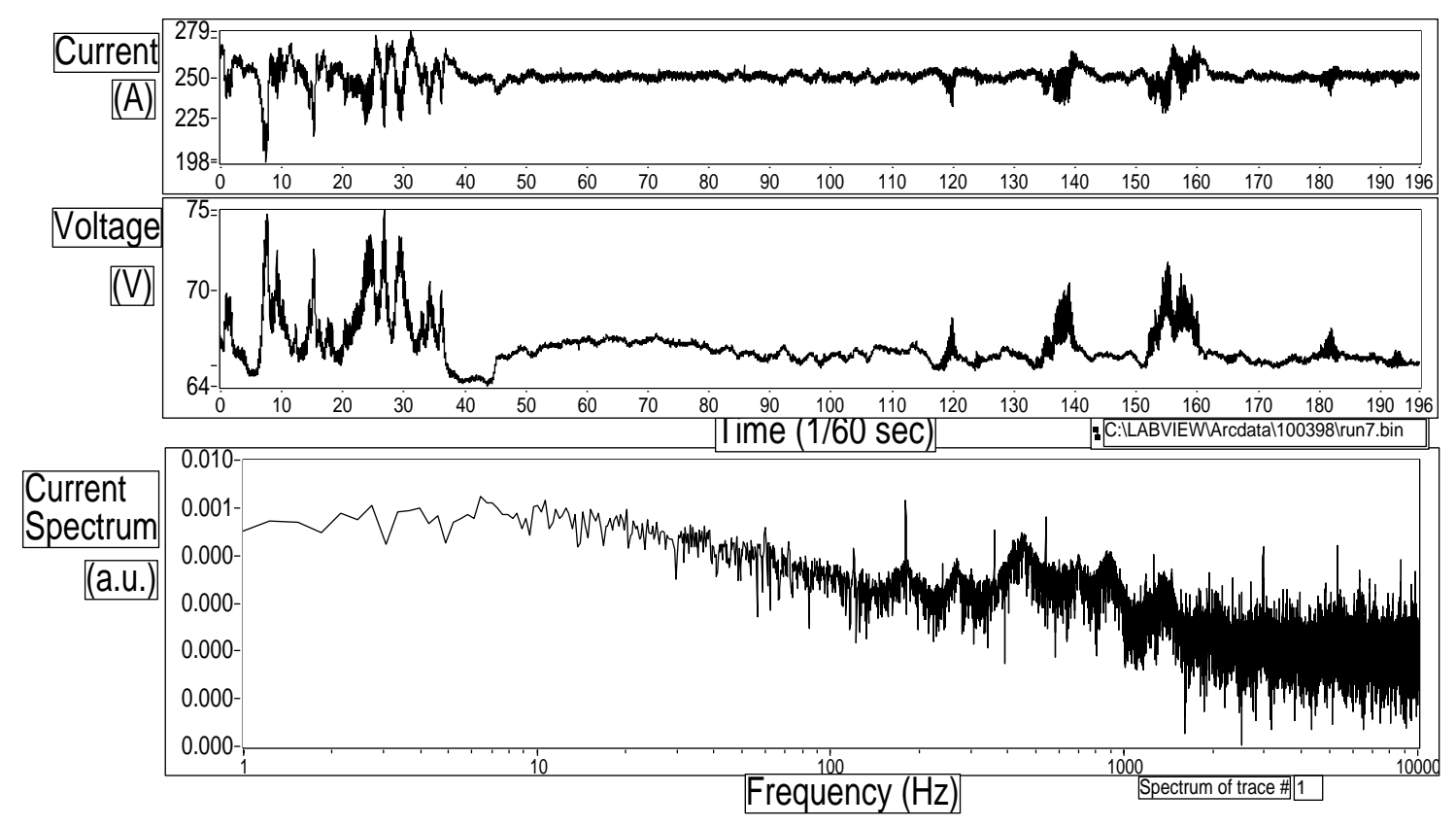

Figure 4.10: Waveforms showing intermittence of the instability bursts. Average arc current is $251 \mathrm{~A}$; electrode gap is $3.6 \mathrm{~cm}$.

through the lower "neck", unstable on the middle button after the lower neck had eroded, stable again on the upper "neck", and became unstable again after reaching the main body of the electrode. Sample images from this sequence are shown in Figure 4.15. The arc current was kept constant throughout. Although the arc length was changing as the cathode was eroding, it was probably not a factor, since the change from stability to instability and vice versa occurred at the point where the cathode diameter change occurred. Furthermore, the arc length has not been found to affect the threshold of the instability, as mentioned in Section 4.2.1.

Besides illustrating the contrast in stability between the narrow and wide sections, the "necked" cathode run also exhibited instability on a wider than usual cathode tip when the arc was attached to the middle section (Figure 4.15(c)). The diameter of that section was approximately $8 \mathrm{~mm}$ at that time, giving a tip area of $\approx 50 \mathrm{~mm}^{2}$, or about twice as large in area as the upper stability threshold for solid cylindrical cathodes. This is most likely due to its higher than normal temperature for cathodes of this size, as will be discussed further in Section 4.4.1. 


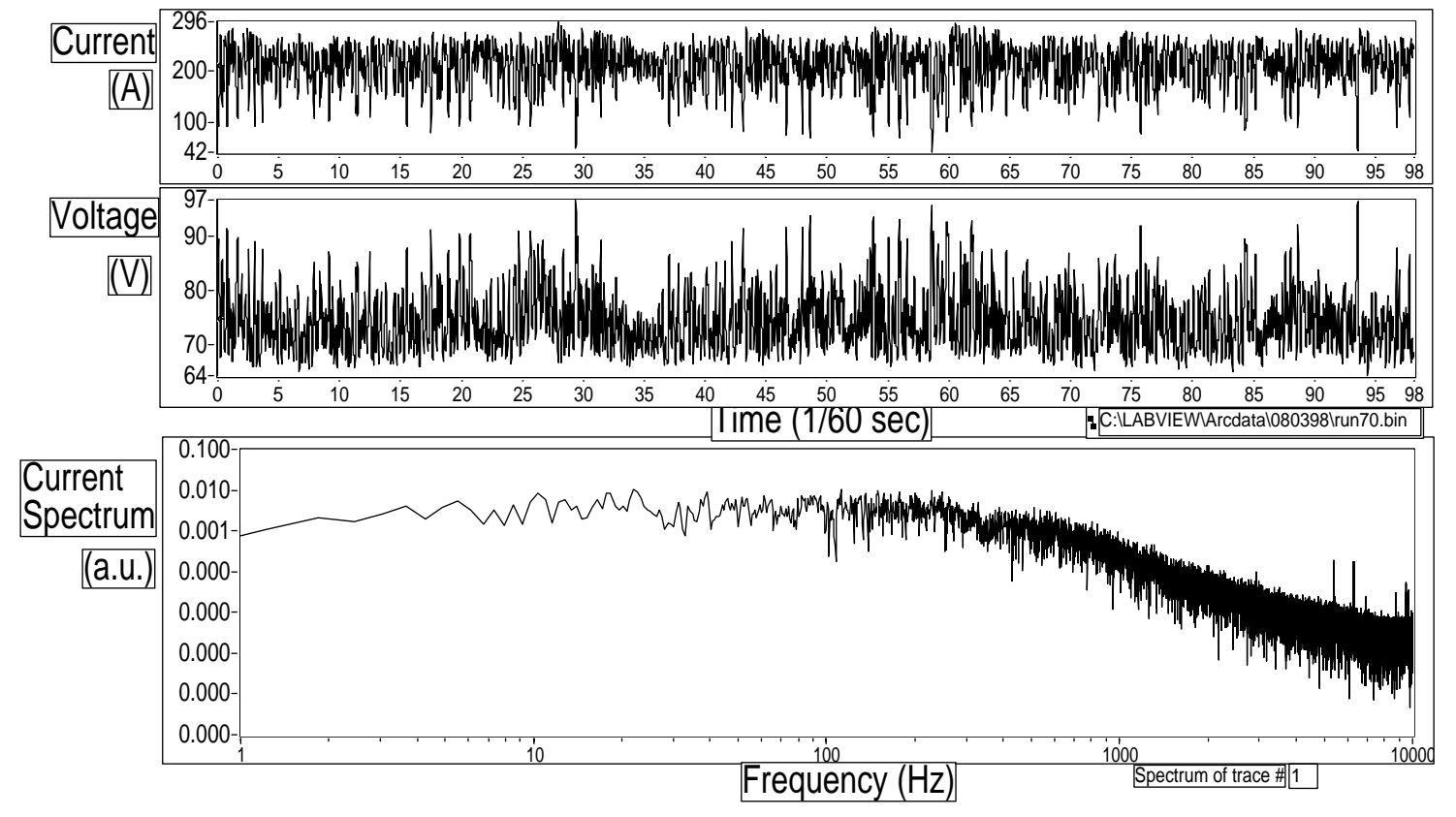

Figure 4.11: Waveforms during a large amplitude instability. Note that the waveforms are no longer simple oscillations, and the current spectrum is broad, with a power law decay $(\approx 2.9$ power $)$. Average arc current is $211 \mathrm{~A}$, with $16 \% \mathrm{RMS}$ fluctuation amplitude.

The geometry of the cathode not only affects the threshold for the instability, but also influences the unstable arc shape. An example of the latter is given in Figure 4.16, which shows images of instability with a "paddle" shaped cathode (Figure 4.13(f) and \# 18 in Table 4.1; also Figure 4.17 shows a photo of this geometry). The amplitude of arc column displacement is significantly greater in the plane of the wide cathode dimension.

Another example of instability behavior influenced by the cathode geometry is shown in the $30 \mathrm{sec}$ sequence in Figure 4.18. The arc is seen to be stable and deflected to the right for a while before suddenly switching from right to left side, being unstable, then becoming stable again. The thermal evolution of the cathode tip is also noticeable by the shift in the brightness of emission. The waveforms acquired during this run are shown in Figure 4.19. As can be seen from the "Arc Position" trace, this back-and-forth switching pattern repeated with approximately 0.8 sec period. 


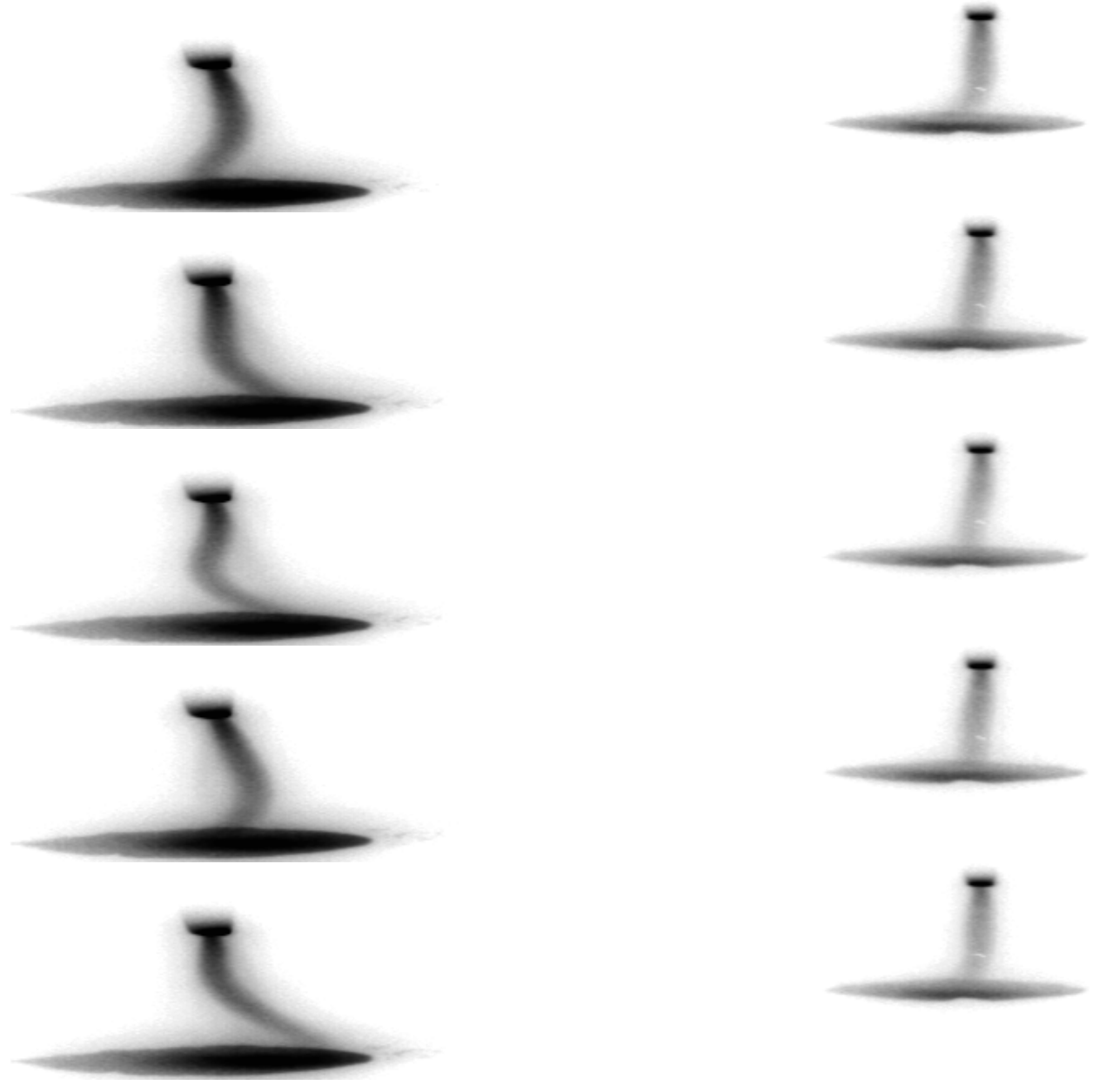

Figure 4.12: The instability is not always three-dimensional. Here the arc is moving in the direct view (left column) and is stationary in the mirror view (right column). The cathode is the standard 3/8" diameter cylindrical rod.

\subsubsection{Cathode spot motion}

Cathode spot motion has been observed in many unstable runs. For example, Figures 4.3 and 4.16 clearly show motion of the arc attachment on the cathode. In order to better examine this effect, a series of runs were taken with a close-up view of the cathode region, at approximately $4 \times$ the spatial resolution of the standard images. 


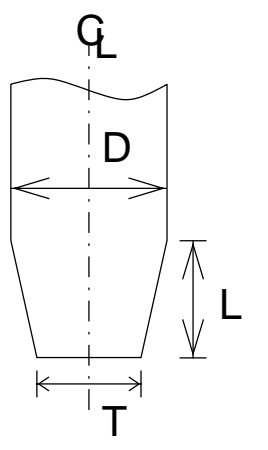

(a)

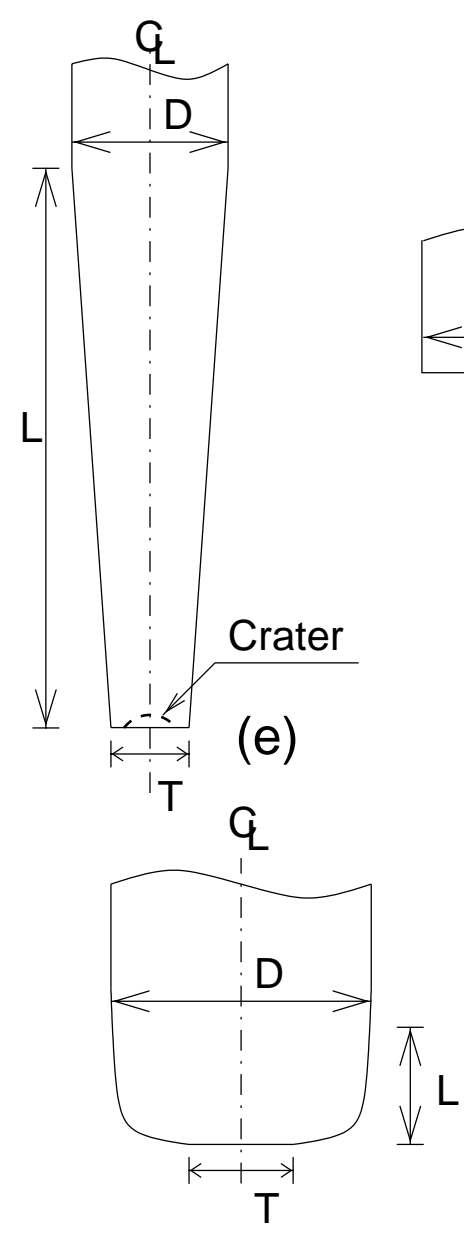

(g)

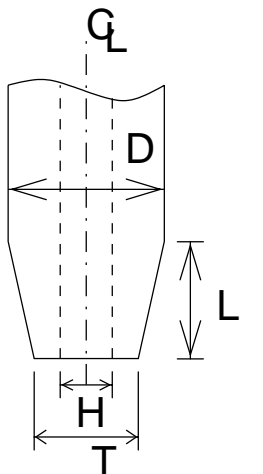

(b)

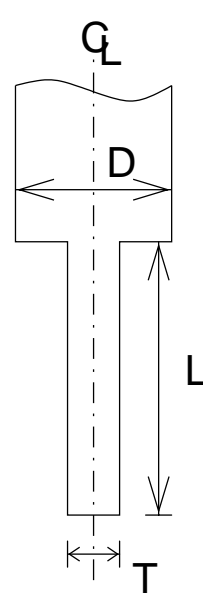

(c)

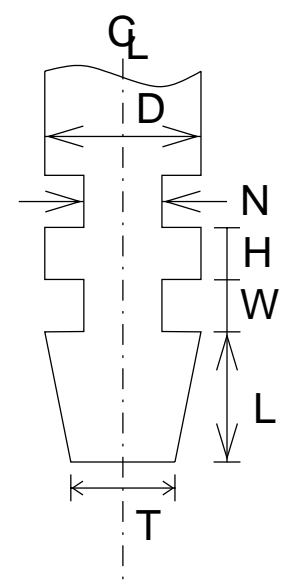

(d)

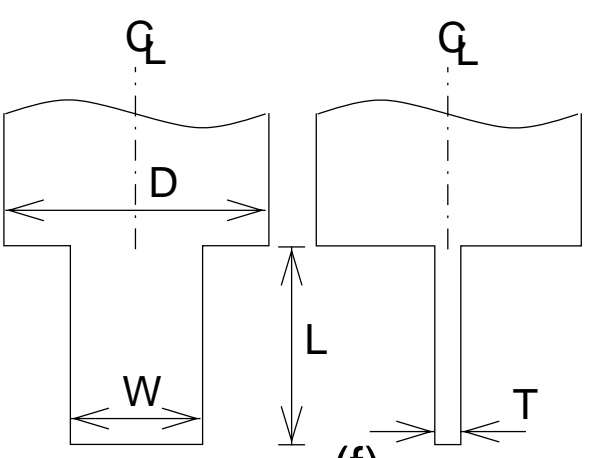

(f)

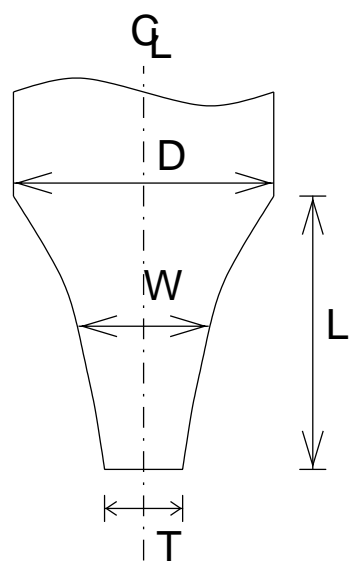

(h)

Figure 4.13: Cathode tips used in stability experiments. (a) standard cathode when new, (b) drilled standard cathode, (c) standard cathode with "necks" machined, (d) standard cathode after a long period of running, (e) "paddle" shaped cathode (face and profile view). The dimensions are given in Table 4.1. 


\begin{tabular}{|c|l||c|c|c|c|c|c|c|c|}
\hline \multirow{2}{*}{$\#$} & \multicolumn{1}{|c||}{ Fig. } & \multicolumn{3}{c|}{ Dimensions (mm unless noted) } & \\
& \multicolumn{1}{|c||}{ Cathode } & 4.13 & $\mathrm{D}$ & $\mathrm{H}$ & $\mathrm{L}$ & $\mathrm{N}$ & $\mathrm{T}$ & $\mathrm{W}$ & Unstable? \\
\hline \hline 1 & Standard New & (a) & $3 / 8^{\prime \prime}$ & - & 7 & - & 6.5 & - & No \\
2 & Standard (15 sec) & (a) & $3 / 8^{\prime \prime}$ & - & 6 & - & 5 & - & Yes \\
3 & McMaster Cu-clad New & (a) & $1 / 2^{\prime \prime}$ & - & 16.8 & - & 6.1 & - & No \\
4 & McMaster Cu-clad Used & (g) & $1 / 2^{\prime \prime}$ & - & 30 & - & 7.9 & - & No \\
5 & Standard Drilled & (b) & $3 / 8^{\prime \prime}$ & 4 & 4.2 & - & 6.2 & - & No \\
6 & Standard Drilled (1 min) & (b) & $3 / 8^{\prime \prime}$ & 4 & 0 & - & 9.5 & - & Yes \\
7 & Standard Drilled & (b) & $3 / 8^{\prime \prime}$ & 2.4 & 7 & - & 6.5 & - & No \\
8 & Standard Drilled (1 min) & (b) & $3 / 8^{\prime \prime}$ & 2.4 & 0 & - & 9.5 & - & Yes \\
9 & Standard Thin & (c) & $3 / 8^{\prime \prime}$ & - & 35 & - & 5 & - & No \\
10 & Standard Thin & (c) & $3 / 8^{\prime \prime}$ & - & 13 & - & 3.3 & - & No \\
11 & Standard Thin & (c) & $3 / 8^{\prime \prime}$ & - & 54 & - & 5.6 & - & Yes \\
12 & Standard Thin & (c) & $3 / 8^{\prime \prime}$ & - & 4 & - & 4.6 & - & No \\
13 & Standard "Necked" & (d) & $3 / 8^{\prime \prime}$ & - & 7.7 & 4.6 & 6.1 & 4.0 & Yes/No \\
14 & Standard Used (>3 min) & (e) & $3 / 8^{\prime \prime}$ & - & 34 & - & 5 & - & Yes \\
15 & Standard Used (>3 min & (e) & $3 / 8^{\prime \prime}$ & - & 20 & - & 6 & - & Yes \\
& & & & & & & & & \\
16 & Standard "Paddle" $250 \mathrm{~A})$ & (f) & $3 / 8^{\prime \prime}$ & - & 43 & - & 5 & 9.5 & Yes \\
17 & McMaster "Paddle" & (f) & $1^{\prime \prime}$ & - & 38 & - & 2.5 & 7.9 & Yes \\
18 & McMaster "Paddle" & (f) & $1^{\prime \prime}$ & - & 43 & - & 2.5 & 8.6 & Yes \\
19 & McMaster "Paddle" & (f) & $1^{\prime \prime}$ & - & 42 & - & 4.2 & 25 & No \\
20 & McMaster "Paddle" & (f) & $1^{\prime \prime}$ & - & 35 & - & 2 & 23.4 & No \\
& & & & & & & & & \\
21 & McMaster Thick min) & (g) & $1^{\prime \prime}$ & - & 9 & - & 10 & - & No \\
22 & McMaster Graded & (h) & $1^{\prime \prime}$ & - & 23 & - & 5 & 13 & Yes \\
23 & McMaster Graded & (h) & $1^{\prime \prime}$ & - & 30 & - & 5.3 & 11.7 & Yes \\
24 & McMaster Graded & (h) & $1^{\prime \prime}$ & - & 30 & - & 6.8 & 11.7 & No \\
\hline
\end{tabular}

Table 4.1: Dimensions of the cathodes used in the stability experiments. For several cathodes, the shape is also given after running for the approximate time shown is parenthesis; otherwise the dimensions are as machined prior to runs. Refer to Figure 4.13 for the drawings. The "Unstable?" column refers to the presence of instability at $250 \mathrm{~A}$.

Figure 4.20 shows such close-up images of the cathode region of arc during large amplitude instability. Shown are single exposure as well as double exposure images. Motion of the point of attachment at the cathode is clearly visible. The double exposure images, with exposures separated by $2 \mathrm{~ms}$, show that the cathode spot motion occurs on the time scale of the instability ( $\approx 1-4 \mathrm{~ms})$. 


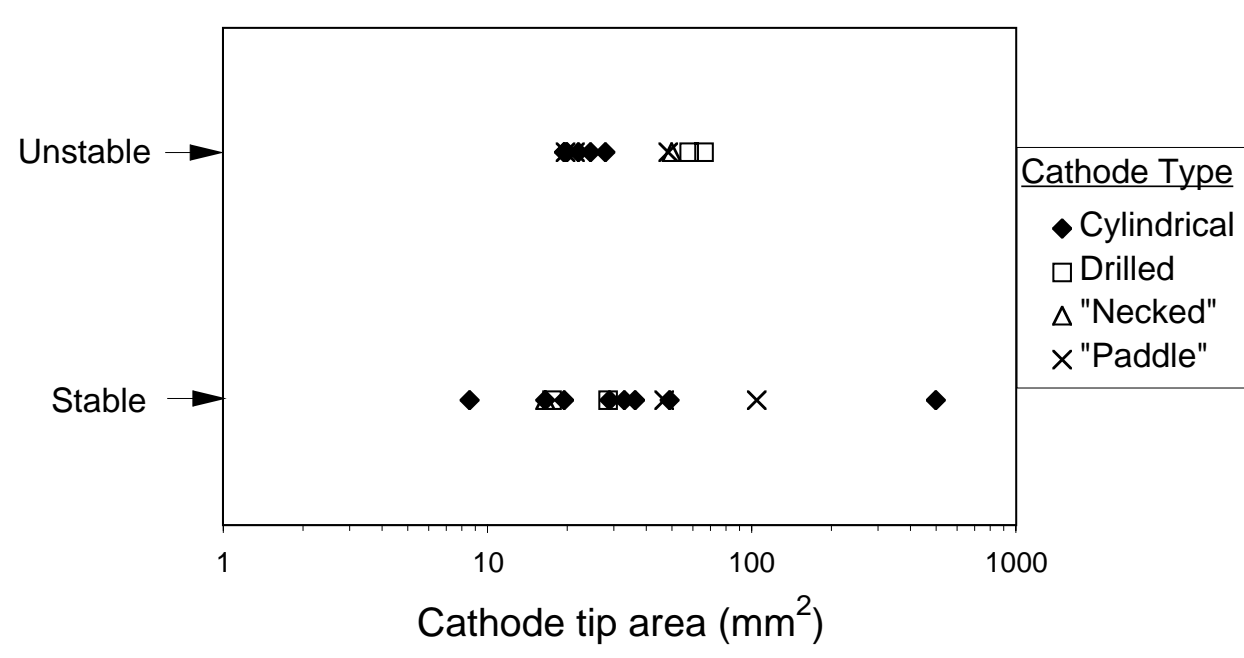

Figure 4.14: Arc stability vs. the cathode tip area for currents up to approximately 250 A. For solid cylindrical cathode tips, the arc is unstable only in the range of $20-28 \mathrm{~mm}^{2}$.

\subsubsection{Erosion rate of the cathode}

The erosion rates of the standard $3 / 8^{\prime \prime}$ cathode during stable and unstable periods are given in Figure 4.21. These measurements were obtained from the video tape record of the runs with the close-up view of the cathode region by measuring the change (recession) in the cathode tip position over time as the electrode position and the arc current remained fixed. The erosion rate during instability is approximately $20 \%$ higher. An erosion rate of $0.06 \mathrm{~mm} / \mathrm{sec}$ corresponds to $\approx 1.2 \times 10^{-3} \mathrm{~cm}^{3}$ of erosion per sec for a $5 \mathrm{~mm}$ diameter cathode tip, or $\approx 2 \times 10^{-6} \mathrm{~cm}^{3}$ per instability period. 


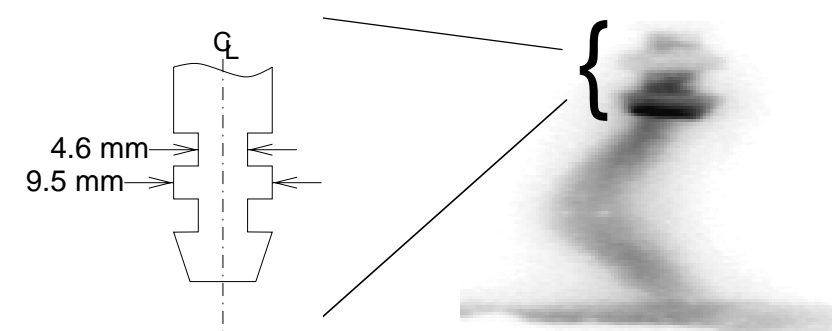

(a)

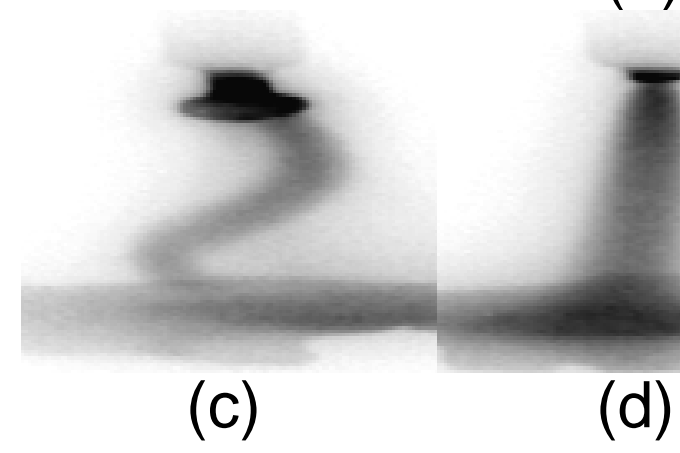

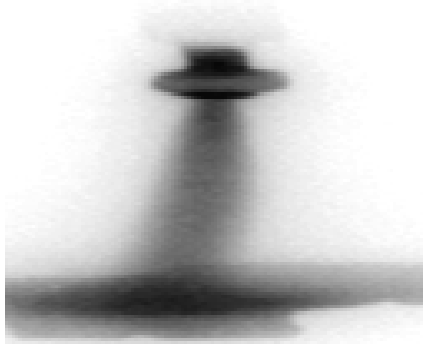

(b)

(e)

Figure 4.15: A time sequence of sample images from the experiment with the "necked" cathode (the initial cathode shape is shown schematically). The arc is (a) unstable on the lower wide section; (b) stable while attached to the remaining part of the lower narrow section; (c) unstable on the middle wide section; (d) stable while burning through the upper narrow section; (e) unstable after the upper narrow neck has been eroded. Arc current is $250 \mathrm{~A}$. Image in (a) is in the mirror view, (b)-(e) are direct view. The horizontal scale is expanded 2.4:1. 


\section{Straight on View \\ (scale: $2.4: 1$ )}

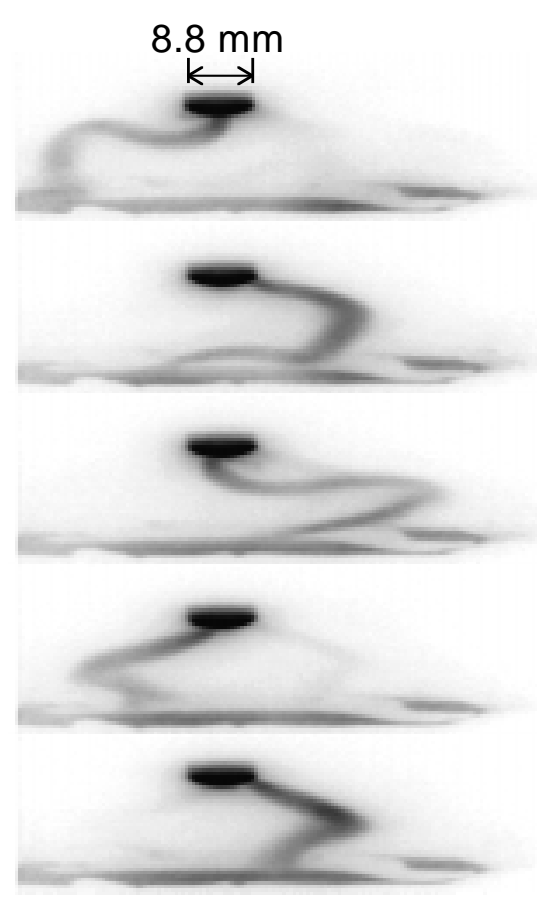

\section{$90^{\circ}$ View in Mirror} (scale 2.3:1)
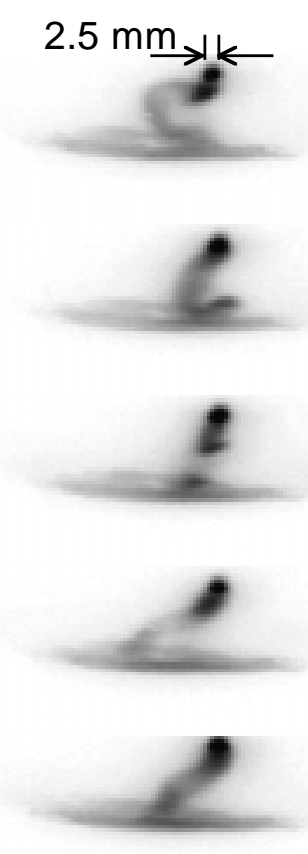

Figure 4.16: Sample pictures of unstable arc during large amplitude oscillation on a "paddle" shaped cathode. Left-hand column shows direct view of the arc; righthand column - a $90^{\circ}$ view in the mirror. Horizontal scale is expanded. Electrode gap is $2.7 \mathrm{~cm}$. Note that the cathode is wider in the direct view plane and that the amplitude of motion of the arc is greater in that plane. Also notable is that the arc attachment is moving along the tip of the cathode. 


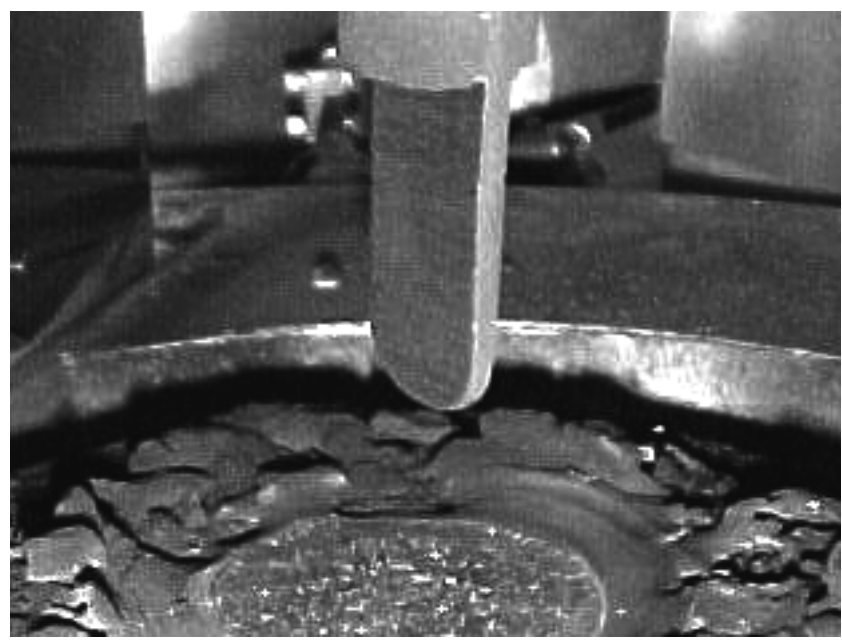

Figure 4.17: A photo of installed "paddle" shaped cathode (\# 19 in Table 4.1) prior to running. Most of the steel anode is also visible at the bottom of the picture. 


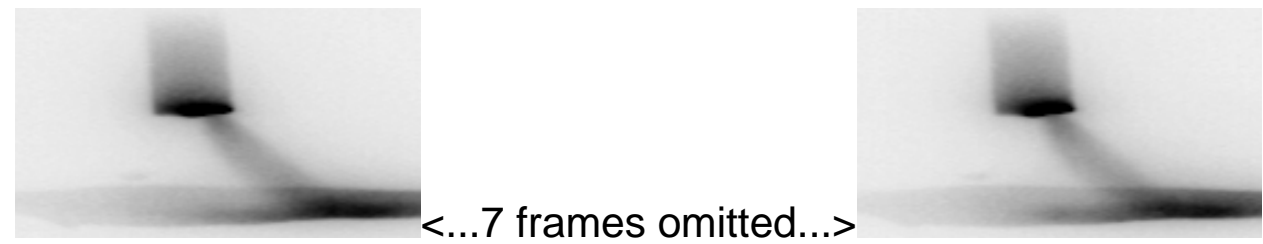

(a)

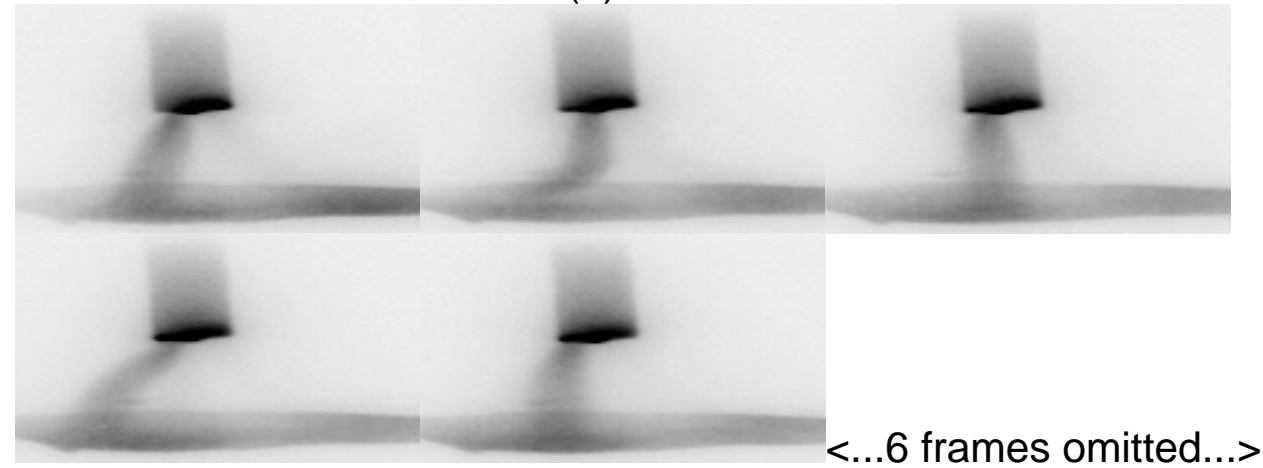

(b)

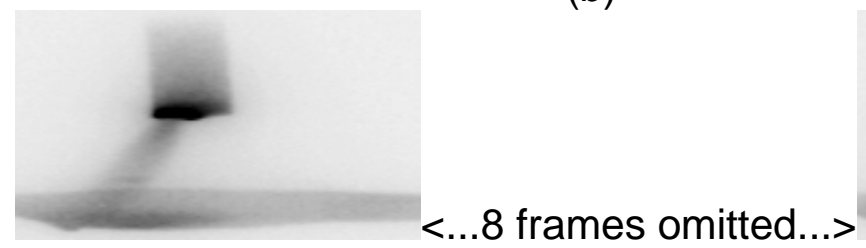

(c)

Figure 4.18: A time sequence of images of a "bistable" arc. Average arc current is $256 \mathrm{~A}$; electrode gap is $2.1 \mathrm{~cm}$. Images are expanded horizontally 2.4:1. 

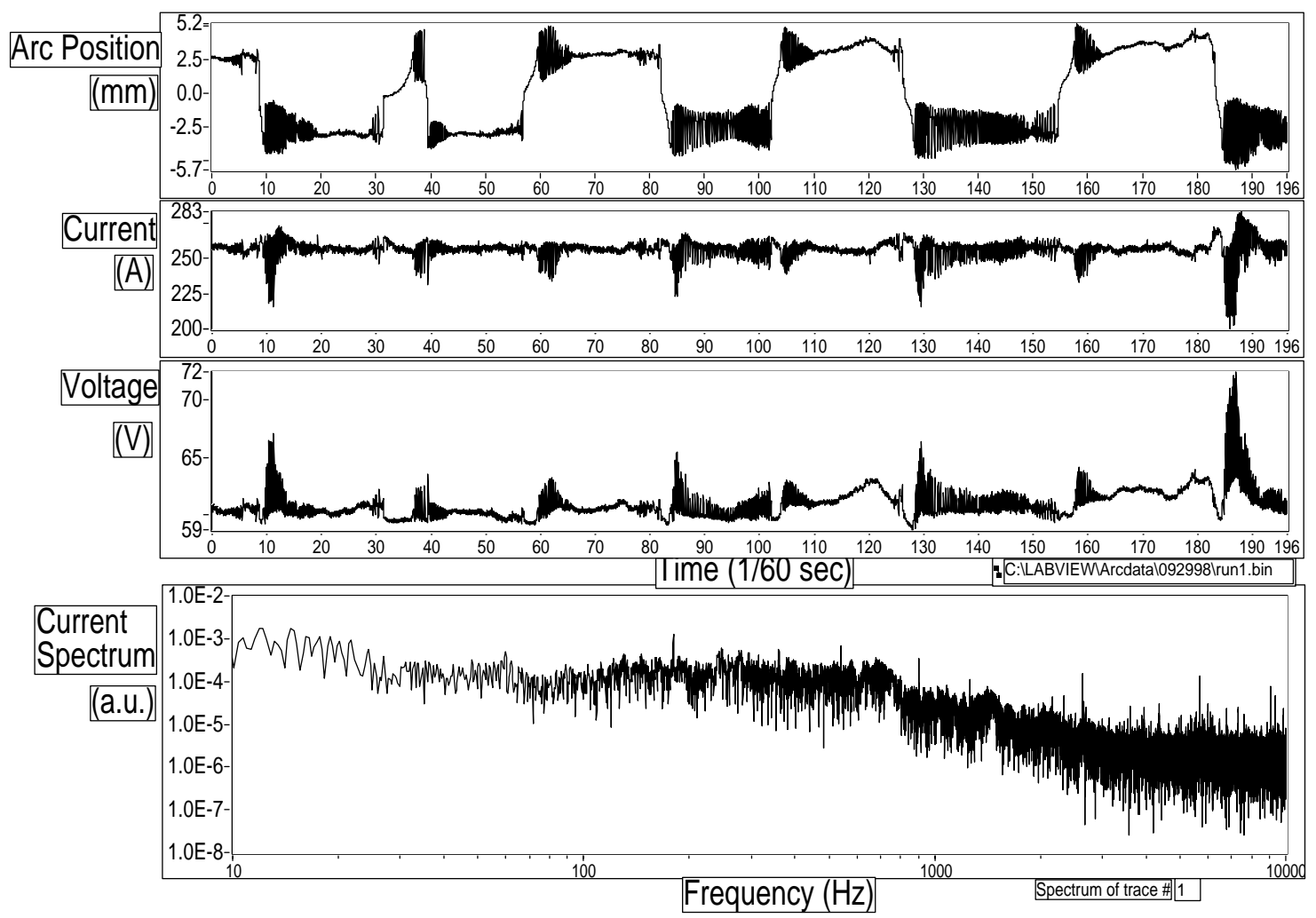

Figure 4.19: The waveforms during the "bistable" arc behavior. The images in Figure 4.18 correspond to fields $0-29$ on the time scale. The switching of the arc from one side of the cathode to the other is evident on the Arc Position trace. 


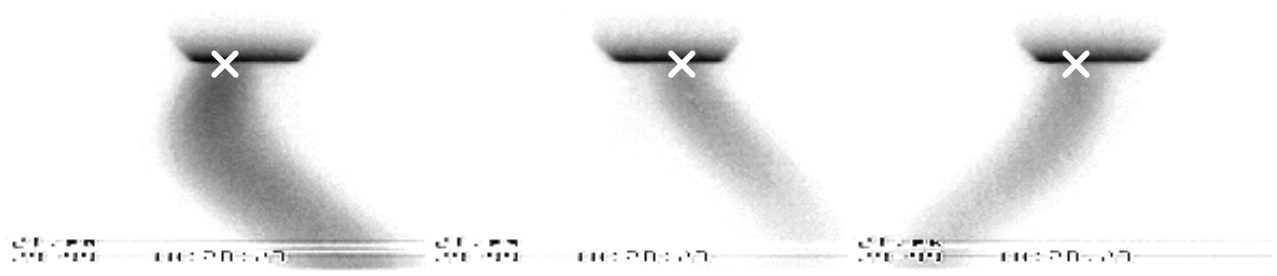

(a) Single exposure
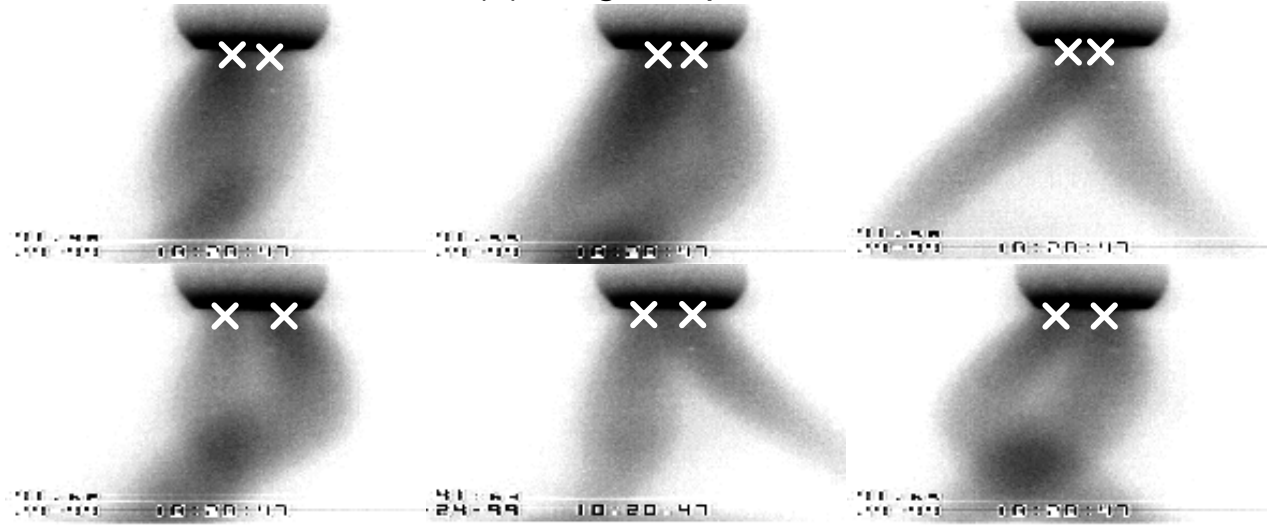

(b) Double exposure

Figure 4.20: Closeup images of the cathode region of the arc during large amplitude instability clearly show motion of the arc along the cathode surface (spot motion). An " $\times$ " marks the approximate cathode spot location. (a) Three fields with single $5 \mu \mathrm{s}$ exposure; (b) six fields with two $5 \mu \mathrm{s}$ exposures per field, with exposures separated by $2 \mathrm{~ms}$. In (b), the two images of the arc column do not overlap completely at the cathode, indicating displacement of the attachment point during the $2 \mathrm{~ms}$ between exposures. Arc current is $250 \mathrm{~A}$. The horizontal scale is expanded 2.3:1. 


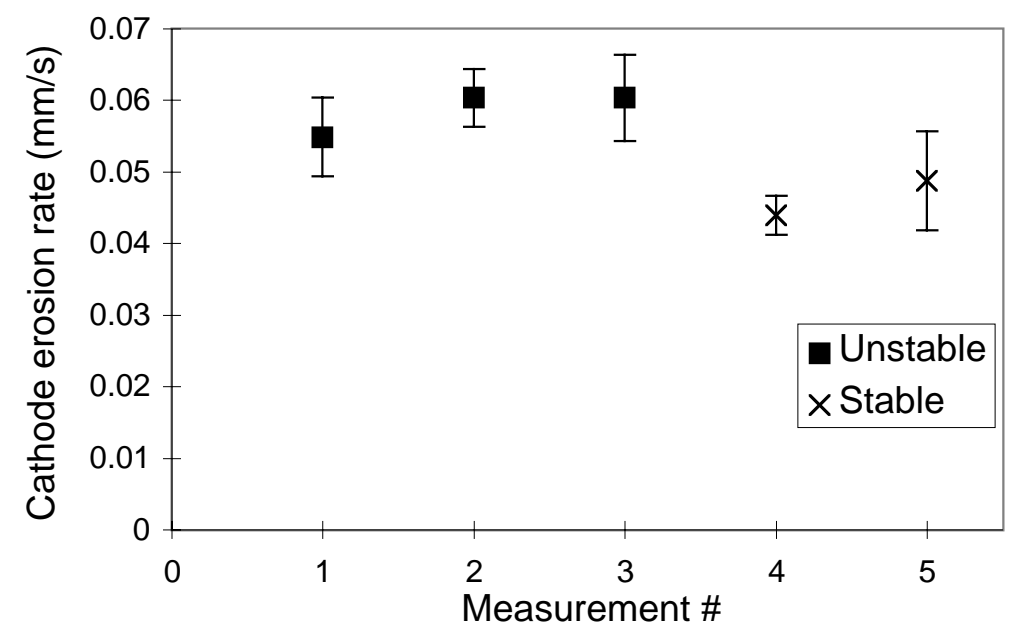

Figure 4.21: Measured erosion rates on standard 3/8" cathodes at $250 \mathrm{~A}$ average arc current. 


\subsection{Model of the Instability}

This section develops a theoretical description of the mechanisms responsible for the instability phenomena described above. A model of the instability derived below in Section 4.3.2 is applied to the experimental observations in Section 4.3.4.

\subsubsection{Mechanism of the instability}

In attempting to discover the mechanism of the instability, several different categories of instabilities have to be considered. Several possible candidates are listed below, together with a discussion of their relevance to the observations.

- Electrical circuit oscillation. Oscillation in the circuit containing the arc power supply and the arc could potentially have produced oscillation of the arc current and voltage. Circuit oscillations alone could not account for the three-dimensional displacement of the arc column nor the observed dependence of the instability on the cathode geometry. Moreover, as discussed in Section 4.2.1, arc column oscillation can appear prior to any oscillation on the current or voltage.

- Shear flow. Instability driven by the shear flows in the jet. These types of instabilities in fluid jets are usually axisymmetric disturbances of the jet column, with the wavelength comparable to the jet diameter [43], but helical modes can also appear under some conditions [53,54]. This type of instability does not explain the observed motion of the cathode spot, nor the occurrence of instability only for a narrow range of cathode diameters (see Section 4.2.2). Additionally, oscillations in a plane observed with "paddle" shaped cathodes are not explained by this mechanism.

- Convective. Convective instabilities can be driven by buoyancy of hot low density gas. The characteristic speeds of buoyancy generated flows are $~$ $\sqrt{g L} \sim 0.7 \mathrm{~m} / \mathrm{s}$, where $g$ is the acceleration of gravity and $L$ is a length scale. Since the jet speed in the experiment is $\sim 20 \mathrm{~m} / \mathrm{s}$, convective effects should not play a role. 


\begin{tabular}{|c|c|c|c|}
\hline & Current-Driven & "Firehose" & Spot Motion \\
\hline Arc Length & $\begin{array}{l}\text { Stable for short } \\
\text { arc }\end{array}$ & $\begin{array}{c}\text { Stable for short } \\
\text { arc }\end{array}$ & $\begin{array}{l}\text { Unstable for } \\
\text { all arc lengths }\end{array}$ \\
\hline Cathode spot & Stationary & Stationary & Moving \\
\hline $\begin{array}{l}\text { Electrode } \\
\text { Geometry }\end{array}$ & Independent & Independent & $\begin{array}{l}\text { Dependent on } \\
\text { cathode geometry }\end{array}$ \\
\hline Current & $\begin{array}{l}\text { More unstable for } \\
\text { higher current }\end{array}$ & $\begin{array}{l}\text { More unstable for } \\
\text { higher currents } \\
\text { (higher jet } \\
\text { speeds) }\end{array}$ & $\begin{array}{c}\text { Depends on spot } \\
\text { motion } \\
\text { mechanism }\end{array}$ \\
\hline Amplitude Growth & Exponential & Exponential & Linear \\
\hline Wavelength & $\begin{array}{l}\text { Fixed by } \\
\text { dispersion } \\
\text { relation }\end{array}$ & $\begin{array}{l}\text { Fixed by } \\
\text { dispersion } \\
\text { relation }\end{array}$ & (jet speed) / (frequency) \\
\hline
\end{tabular}

Table 4.2: Expected characteristics for a current-driven column instability, firehose instability, and a spot motion instability. The shaded characteristics are confirmed in the experiment.

- Anode effects. Effects such as the current distribution in the anode and vapor generation in the anode spot do not explain the dependence of the instability on the cathode geometry.

- "Firehose." This type of fluid instability, discussed in Ref. 55 in connection to astrophysical jets, occurs for jets with a confining boundary, such as a hose or a dense surrounding medium. It is also invoked as a destabilizing mechanism in Ref. 31 and to qualitatively explain the arc instability in Ref. 33, with the magnetic field of the arc serving as the confining boundary.

- Current driven. Current driven "kink" instabilities [56] are a strong candidate since such instabilities are known to occur in z-pinches, where the geometry is similar to that of the arc.

- Cathode spot motion. Cathode spot motion is observed in the experiment and can account the observed arc shape, as will be shown below.

The last three possible instability mechanisms appear to be the most likely candidates. Their expected characteristics are are compared in Table 4.2, with the experimentally confirmed characteristics shaded.

As can be seen from the Table, a current-driven column and "firehose" type instabilities have characteristics that conflict with the experimental observations of the instability. Neither one can explain the occurrence of the instability for 
only a range of cathode diameters nor the linear spatial growth of the arc shape. The cathode spot motion model, on the other hand, is able to account for the observations and is developed below. Further discussion of possible mechanisms of instability is given in Section 4.6.3. Discussion of the possible causes of the cathode spot motion is deferred until Section 4.4.

\subsubsection{Derivation of arc shape based on spot motion model}

The notion that the cathode spot motion can be an instability mechanism and can influence the shape of the arc column via the cathode jet is mentioned by Bowman in Ref. 2. Guillery and Finkelnburg [11] suggest that cathode spot mobility is necessary for the formation of a helical arc shape. However, no quantitative implications of spot motion for the arc shape are found in the literature. This section gives a derivation of the shape of the arc column due to the motion of the cathode spot.

The assumptions used in this model of the unstable arc shape are the same as made in the derivation of the driven arc motion Chapter 3, Section 3.3.1, except for the conditions at the cathode. Assumption 6 is relaxed to allow the cathode spot to move over the cathode surface. To simplify the derivation, the cathode surface will be assumed flat. As before, the cathode jet is assumed to start out normal to the cathode surface. With the absence of any external magnetic field, and once again neglecting the self magnetic field effects, the force balance equation is:

$$
\frac{D \vec{v}}{d t}=0
$$

Letting the z-axis be along the equilibrium arc axis, with positive direction away from the cathode. Let

$$
\vec{v}=v \hat{z}+\tilde{v}_{x} \hat{x}+\tilde{v}_{y} \hat{y},
$$


where $v$ is the jet speed, and $\tilde{v}_{x}$ and $\tilde{v}_{y}$ are the perturbed transverse velocity components. Expanding convective derivatives and linearizing, get:

$$
\frac{D \tilde{v}}{d t}=\frac{\partial}{\partial t}\left(\tilde{v}_{x} \hat{x}+\tilde{v}_{y} \hat{y}\right)+v \frac{\partial}{\partial z}\left(\tilde{v}_{x} \hat{x}+\tilde{v}_{y} \hat{y}\right)=0
$$

As can be seen from the above equation, in the linear approximation the equation of motion separates into the $\mathrm{x}$ and $\mathrm{y}$ components. The equation can thus be solved for motion in a plane and the full 3D shape recovered by adding out-of-the-plane motion at the end. Choosing the $\mathrm{y}-\mathrm{z}$ plane, expanding $\tilde{v}_{y}$ as the convective derivative of transverse displacement $\xi$ and linearizing, obtain:

$$
\tilde{v}_{y}=\frac{\partial \xi}{\partial t}+v \frac{\partial \xi}{\partial z}
$$

Substituting the above into Eq. (4.3):

$$
\frac{\partial^{2} \xi}{\partial t^{2}}+2 v \frac{\partial^{2} \xi}{\partial t \partial z}+v^{2} \frac{\partial^{2} \xi}{\partial z^{2}}=0
$$

Equation 4.5 is identical to equation 3.4 except for the absence of the forcing term. Assuming that the cathode surface is flat and that the equilibrium arc axis is normal to it, let the position of the cathode spot in the y-z plane be $y=f(t), z=0$. The initial conditions at the cathode are now a function of time: $\left.\xi(z, t)\right|_{z=0}=f(t)$ and $\left.\frac{\partial \xi}{\partial z}\right|_{z=0}=0$. Fourier transforming in time and incorporating these initial conditions in the Laplace transform in $\mathrm{z}$, the solution is:

$$
\xi(z, t)=f\left(t-\frac{z}{v}\right)+\frac{z}{v} f^{\prime}\left(t-\frac{z}{v}\right)
$$

The shape of the arc column is thus determined by the trajectory of the spot on the cathode through the effect of the cathode jet.

If the cathode spot is assumed to gyrate on the cathode at a particular angular frequency $\omega$ on an ellipse with major and minor radii of $2 a$ and $2 b$, its trajectory on the flat cathode surface can be parametrized as $a \cos (\omega t) \hat{y}+b \sin (\omega t) \hat{x}$. Its motion in the $\mathrm{y}-\mathrm{z}$ plane would then be simple harmonic: $f(t)=a \cos (\omega t)$. 


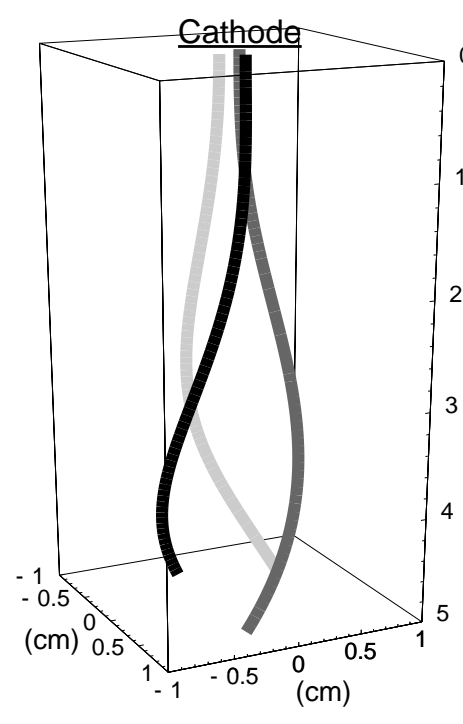

$\underline{400 \mathrm{~Hz}}$

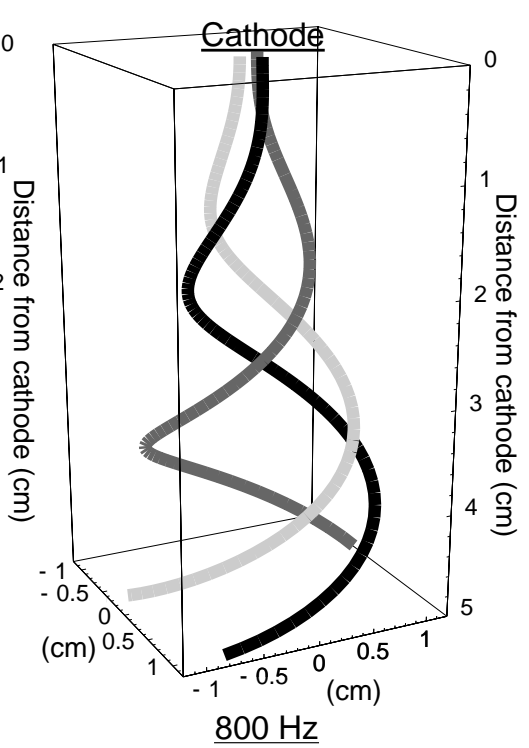

Figure 4.22: Derived arc shape for circular gyration of the spot on the cathode for two frequencies of gyration: $400 \mathrm{~Hz}$ and $800 \mathrm{~Hz}$. Arc shape is shown at three instances in time. Jet speed is $30 \mathrm{~m} / \mathrm{s}$; radius of gyration is $1.5 \mathrm{~mm}$. Horizontal scale is expanded.

Using Eq. (4.6), the arc shape as seen in the y-z plane would be:

$$
\xi(z, t)=a\left[\cos \left(\omega t-\frac{\omega z}{v}\right)-\frac{z \omega}{v} \sin \left(\omega t-\frac{\omega z}{v}\right)\right]
$$

In the $\mathrm{X}-\mathrm{z}$ plane, the motion would be $90^{\circ}$ out of phase. Combining the two, one obtains the $3 \mathrm{D}$ arc shape:

$$
\begin{aligned}
& \vec{\xi}(z, t)=a\left[\cos \left(\omega t-\frac{\omega z}{v}\right)-\frac{z \omega}{v} \sin \left(\omega t-\frac{\omega z}{v}\right)\right] \hat{y}+ \\
& b\left[\sin \left(\omega t-\frac{\omega z}{v}\right)+\frac{z \omega}{v} \cos \left(\omega t-\frac{\omega z}{v}\right)\right] \hat{x}
\end{aligned}
$$

An example plot of Eq. (4.8) is shown in Figure 4.22.

As can be seen in Eq. (4.8), the arc shape has no dependence on mass density, and is determined by the spot motion amplitude $a$, the frequency $\omega$, and the average jet velocity $v$. 
Eq. (4.8) can be written in dimensionless form by defining $\zeta \equiv z \omega / v, \tau \equiv \omega t$, and $\chi \equiv \xi \omega / v$ as before, as well as $A \equiv a \omega / v$ and $B \equiv b \omega / v$ :

$$
\vec{\chi}(\zeta, \tau)=A[\cos (\tau-\zeta)-\zeta \sin (\tau-\zeta)] \hat{y}+\begin{aligned}
& \quad B[\sin (\tau-\zeta)+\zeta \cos (\tau-\zeta)] \hat{x}
\end{aligned}
$$

For short arc lengths, when $\zeta \ll 1$, Eq. (4.9) can be Taylor expanded:

$$
\begin{aligned}
\vec{\chi}(\zeta, \tau)=A \hat{y}[\cos (\tau)(1+ & \left.\left.\frac{\zeta^{2}}{2}\right)+\frac{\zeta^{3}}{3} \sin (\tau)\right] \\
& +B \hat{x}\left[\sin (\tau)\left(1+\frac{\zeta^{2}}{2}\right)+\frac{\zeta^{3}}{3} \cos (\tau)\right]+\mathcal{O}(\zeta)^{4}
\end{aligned}
$$

The arc shape for short electrode gaps is thus parabolic with a cubic correction important for $\hat{y}$ component when phase $\tau$ is an odd multiple of $\pi / 2$ and for $\hat{x}$ component when $\tau$ is a multiple of $\pi$.

\subsubsection{Arc length variation due to spot motion}

Since the voltage across a stable arc is proportional to the arc length (Figure 2.11), it is reasonable to expect that the voltage fluctuations observed during the instability are at least in part caused by the arc length variation. From the arc shape predicted by the model, the expected arc length variation can be obtained and compared to the one seen in experiments.

Following the derivation of Section 3.3.3, and assuming the arc shape is described by Eq. (4.8), the arc length as a function of the phase $\tau$ and the dimensionless electrode gap $H \equiv \omega h / v$ can be obtained:

$$
L(H, \tau)=\frac{v}{\omega} \int_{0}^{H} \sqrt{1+\zeta^{2}\left[A^{2} \cos ^{2}(\tau-\zeta)+B^{2} \sin ^{2}(\tau-\zeta)\right]} d \zeta
$$


If the cathode spot moves strictly on a circle $(A=B)$, the arc length is not a function of time and, normalized by the electrode gap $h$, is given by:

$$
\frac{L(H)}{h}=\frac{1}{2 A H} \ln \left(A H+\sqrt{1+(A H)^{2}}\right)+\frac{1}{2} \sqrt{1+(A H)^{2}}
$$

For $A H \ll 1$, Eq. (4.12) can be expanded as:

$$
\frac{L(H)}{h}=1+\frac{(A H)^{2}}{6}+\mathcal{O}(A H)^{4}
$$

The $\%$ arc length increase for unstable arc thus grows as the square of electrode gap and the square of spot motion amplitude.

In general, when $A \neq B$, Eq. (4.11) is non-integrable. Numerical integration for different values of $h, A$, and $B$ shows that $L(\tau) \sim \cos (2 \tau)$, or, in other words, that the arc length varies at twice the frequency of spot motion $\omega$.

For $\zeta^{2} A^{2} \ll 1$ and $\zeta^{2} B^{2} \ll 1$, the square root in Eq. (4.11) can be Taylor expanded and integrated, resulting in:

$$
\begin{aligned}
& \frac{L(H, \tau)}{h}=1+\frac{\left(A^{2}+B^{2}\right)^{2} H^{2}}{12}+\frac{\left(A^{2}-B^{2}\right)}{16 H} \times \\
& \quad\left[2 H \cos 2(H-\tau)-\left(1-2 H^{2}\right) \sin 2(H-\tau)+\sin (2 \tau)\right]+\mathcal{O}\left(\left(A^{2}+B^{2}\right)^{2} H^{4}\right)
\end{aligned}
$$

As can be seen from this equation, the amplitude of arc length fluctuation is proportional to the difference of the squares of the spot motion amplitudes in the two planes and thus is higher for more elliptical motion of the spot on the cathode. Taking a time average over a period of the above, get the average arc length:

$$
\frac{\langle L(H, \tau)\rangle_{\tau}}{h} \approx 1+\frac{\left(A^{2}+B^{2}\right)^{2} H^{2}}{12}
$$




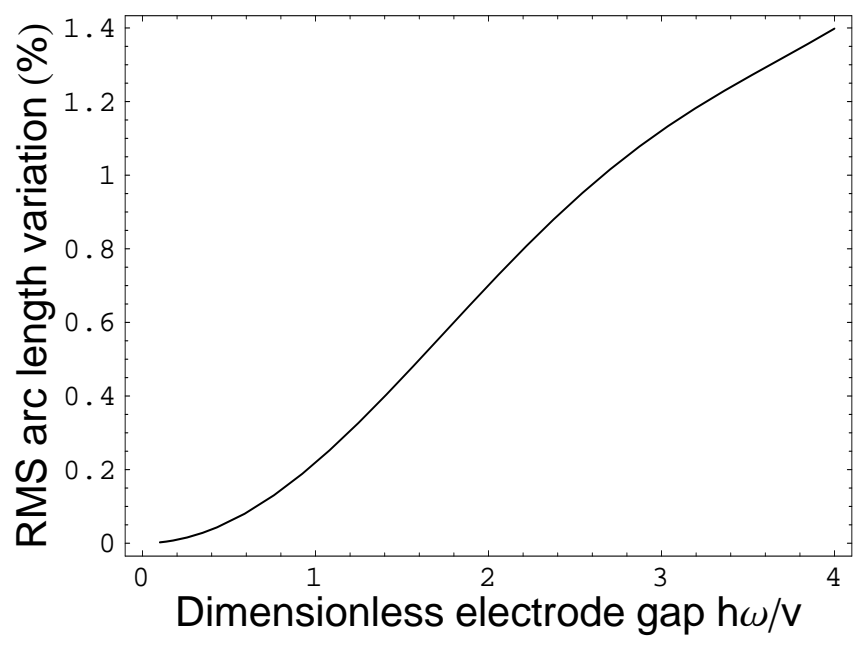

Figure 4.23: The RMS length variation increases with the electrode gap. The range on the abscissa corresponds to $0-5 \mathrm{~cm}$ electrode gap for jet speed of $25 \mathrm{~m} / \mathrm{s}$ and fundamental frequency of $500 \mathrm{~Hz}$. Spot motion amplitudes assumed are $a=0.2 \mathrm{~cm}$ and $b=0.16 \mathrm{~cm}$.

Subtracting this average value from Eq. (4.14) and taking the RMS, get the RMS fluctuation level of the arc length:

$$
\text { rms fluctuation }=\frac{\left|A^{2}-B^{2}\right|}{16 H} \sqrt{1+2 H^{4}-\left(1-2 H^{2}\right) \cos (2 H)-2 H \sin 2 H}
$$

A plot of this equation in Figure 4.23 shows that the \% RMS length variation increases with the electrode gap. Thus, the increase in the current fluctuation amplitude with electrode gap seen in Figure 4.2 is expected just on the basis of geometrical considerations if the arc shape is due to spot motion.

\subsubsection{Application of the Spot Motion Model to the Insta- bility Experiments}

Arc instability data is analyzed on the basis of the spot-motion model derived above. As was done in the applied transverse magnetic field case, the derived equation for arc shape is fitted to the arc shape obtained from arc images. Since 
the arc shapes obtained in experiment are 2-D projections of what is, in general, a 3-D arc shape, Eq. (4.7) was used to analyze the 2D images of the direct view and the $90^{\circ}$ mirror view of the arc. As in the applied transverse magnetic field case, there are two free parameters in the model: the spot motion amplitude $a$ and the jet velocity $v$. For a particular oscillation frequency $\omega$, the first one determines the amplitude of the arc oscillation, the second - the wavelength.

As in the applied magnetic field case, a fit parameter $\theta$ has been added to account for any small angle (typically $<5^{\circ}$ ) offset of the jet from the electrode axis. This takes into account, for example, any deviation of the cathode endface from the horizontal of the camera's field of view. Unlike in the applied field case, however, where the phase of the applied field was known precisely, the phase of the spot motion $\tau$ is a fit parameter as well.

The direction of Earth's magnetic field is transverse to the arc current, which puts a small parabolic deflection bias on the arc column. From the data on arc deflection in transverse magnetic field (Chapter 3), the maximum deflection occurs at the anode, and for the 0.4 Gauss Earth's magnetic field is $1 \mathrm{~mm}$ for a $4 \mathrm{~cm}$ long arc. This deflection is much smaller than the $\approx 1 \mathrm{~cm}$ arc column displacement due to the instability and is neglected in the model.

The arc shape obtained from the camera images has the origin set at the brightest location - the cathode spot. The fit function must therefore be zeroed to the spot location by subtracting $\xi(z=0)=a \cos (\tau)$ from Eq. (4.7). The form of the equation that is fitted to the arc shape then becomes:

$$
\xi_{\text {fit }}(z)=a\left[\cos \left(\tau-\frac{\omega z}{v}\right)-\frac{z \omega}{v} \sin \left(\tau-\frac{\omega z}{v}\right)-\cos (\tau)\right]+\theta z
$$

with $a, v, \tau$, and $\theta$ as fit parameters.

Since a single frequency of oscillation $\omega$ was assumed in deriving the above equation, the fits are done for runs where the frequency of oscillation is still distinct, unlike the large amplitude runs such as those of Figure 4.11 and Figure 4.16. Also, because of the additional phase parameter $\tau$, the fits had to be done for arcs sufficiently long or the frequency of oscillation sufficiently high to have at least half 


\begin{tabular}{|c|c|c|c|}
\hline $\begin{array}{c}\text { RMS f } \\
\text { current } \\
(\%) \\
\end{array}$ & $\begin{array}{l}\text { uation: } \\
\text { voltage } \\
(\%) \\
\end{array}$ & $\begin{array}{c}\text { Fundamental } \\
\text { frequency } \\
(\mathrm{Hz})\end{array}$ & $\begin{array}{l}\text { Spot motion } \\
\text { amplitude } \\
\pm 0.003(\mathrm{~cm})\end{array}$ \\
\hline 1.67 & 1.0 & 460 & 0.066 \\
\hline 1.81 & 1.1 & 622 & 0.072 \\
\hline 17.0 & 6.7 & broad & 0.148 \\
\hline 11.6 & 5.45 & broad & 0.148 \\
\hline
\end{tabular}

Table 4.3: Measured spot motion amplitudes during instability for four runs with the standard $3 / 8^{\prime \prime}$ cathode. Average arc current is $255 \mathrm{~A}$.

a wavelength on the arc column in order to uniquely determine the spot motion amplitude, phase and the jet speed.

Examples of typical fits using Eq. (4.17) are shown in Figure 4.24. In majority of cases, good agreement can be seen, with the fit correlation coefficient R generally 0.94 or higher, and typically above 0.98 (Figure 4.25). The example of Figure 4.17(c) illustrates a case where a good fit was not obtained. Such column displacements can be due to a non-harmonic displacement of the cathode spot and/or motion of the cathode spot outside the flat cathode tip area assumed in the model.

The amplitudes of spot motion inferred from the fits can be compared to the amplitudes measured from close-up, high resolution images of the cathode region, such as shown in Figure 4.20(a). Table 4.3 gives the spot motion amplitudes for four runs at $255 \mathrm{~A}$ average arc current. The amplitude for each run was obtained by finding the center of the luminous arc column immediately below the cathode for each of the 30 images per run and taking the distance between the extreme left and extreme right positions of the column center as being twice the amplitude of the spot motion.

Figure 4.26(a) has superimposed on it the four measured amplitudes and the amplitudes inferred from the fits of the model. The plot confirms that the spot motion amplitudes required by the model to describe the observed arc shape are fairly close to those actually present in the experiments. Figure 4.26(b) gives the 


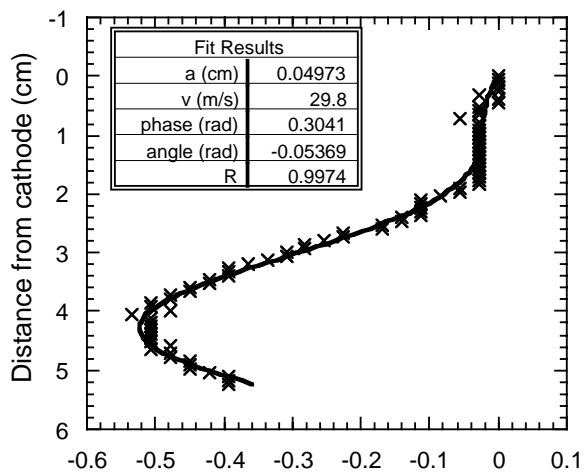

(a)

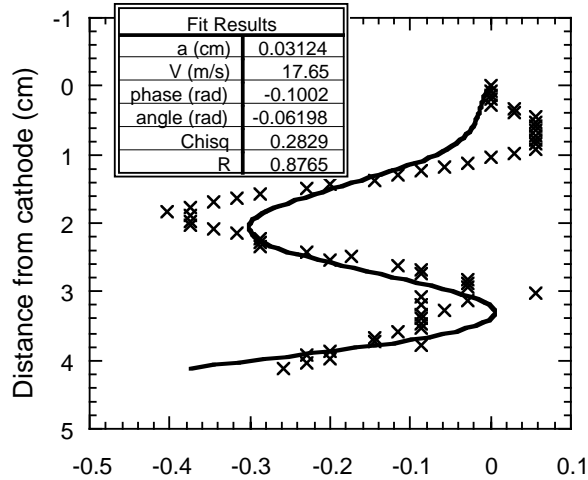

(c)

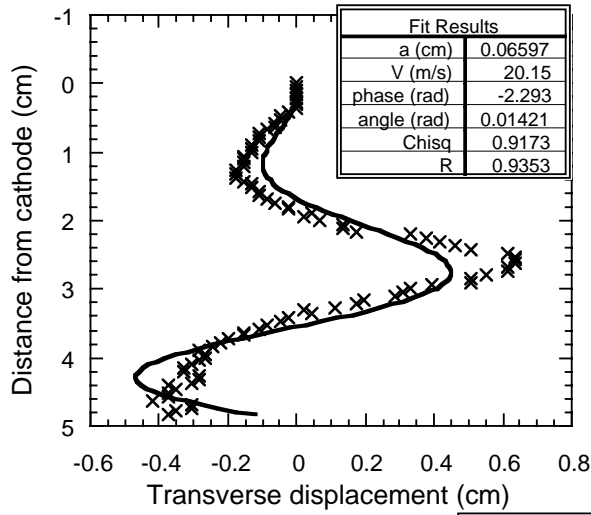

(e)

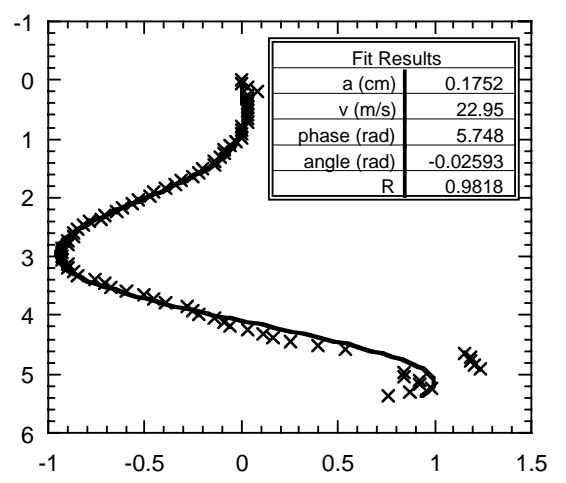

(b)

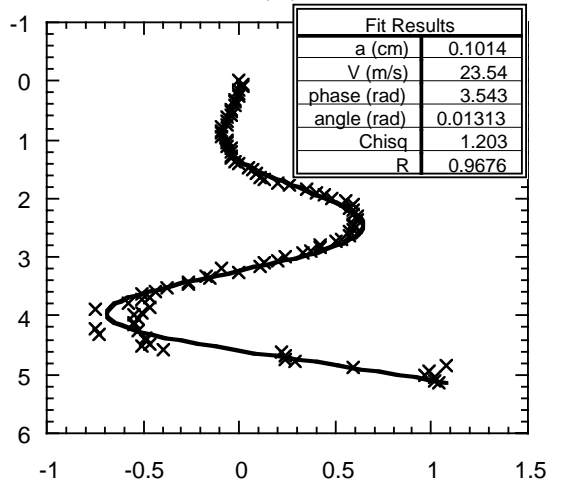

(d)

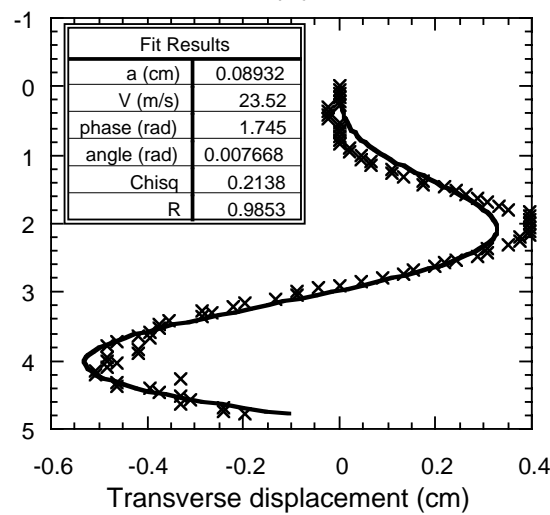

(f)

Figure 4.24: Examples of fits of the model to the experimentally obtained arc shape during instability. The instantaneous arc current and the frequency of oscillation, respectively, are: (a)250 A, $575 \mathrm{~Hz}$; (b) $230 \mathrm{~A}, 520 \mathrm{~Hz}$; (c) $212 \mathrm{~A}, 650 \mathrm{~Hz}$; (d) $220 \mathrm{~A}, 780 \mathrm{~Hz}$; (e) $197 \mathrm{~A}, 650 \mathrm{~Hz}$; (f) $222 \mathrm{~A}, 600 \mathrm{~Hz}$. In (a)-(c) the data is from the mirror view of the arc; in (d)-(f) from the direct view. Note: the horizontal scales are different. 


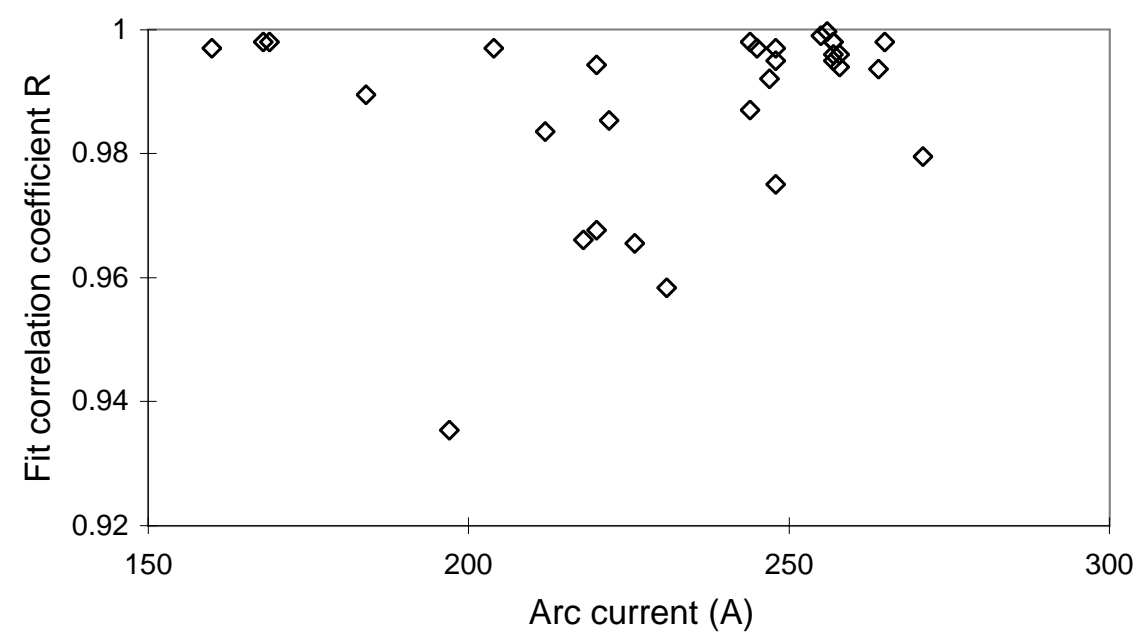

Figure 4.25: Good fits using the cathode spot motion model are obtained for the full range of current studied.

maximum arc displacement at $4 \mathrm{~cm}$ from the cathode as inferred from the fits to arc shape for the same data set.

Figure 4.27 compares the jet speeds inferred from the fits to the jet speeds inferred from the applied transverse magnetic field experiments, described in the previous Chapter. The inferred jet speed increases with the arc current, as expected from Maecker's scaling (Section 3.4.2) and has approximately the same slope as in the applied field experiments. The approximate agreement of the values indicates that the jet speed required for the spot motion model to fit the instability data is consistent with that inferred from the applied field experiments, as expected.

It should be emphasized that this one-dimensional model can only be applied to describe the low amplitude arc column displacements, such as in Figure 4.24, and cannot be used to describe the large amplitude displacements such as seen in Figure 4.16. It is plausible that these cases do not lie outside the framework of the spot motion model, but require a more sophisticated treatment for quantitative analysis than the one-dimensional approximation used here. 

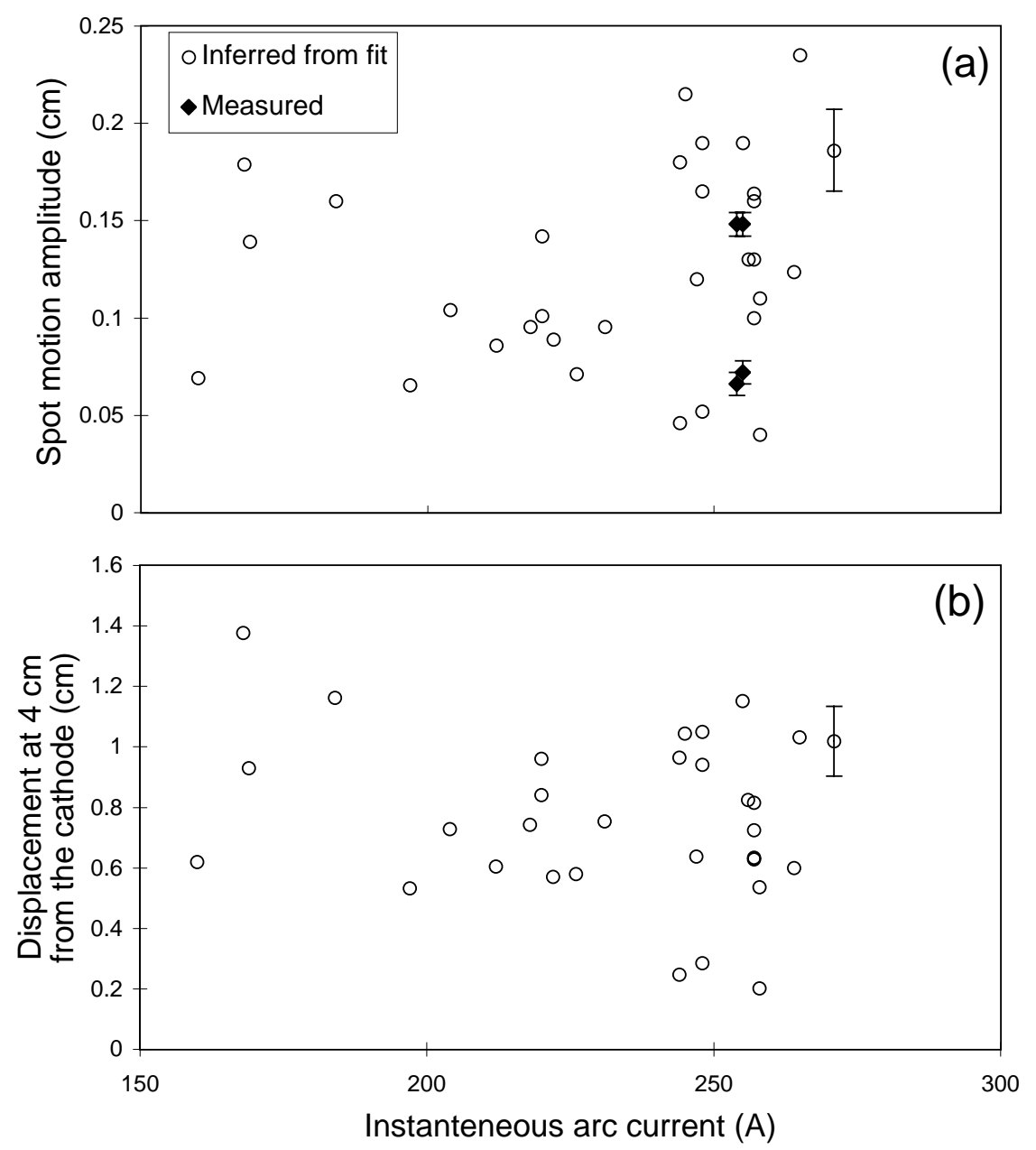

Figure 4.26: (a) The spot motion amplitude inferred from the fits of the model to unstable arc shape for instantaneous arc current. Also plotted are the measured amplitudes for four runs. The error bars on the measured amplitudes reflect the image resolution, while the error bars shown on several of the inferred amplitudes are typical standard errors from the fits. (b) Maximum arc displacement at $4 \mathrm{~cm}$ from the cathode as inferred from the fits to arc shape for the same data set as in (a). No trend vs. current is apparent; however, a trend may be masked by the scatter in spot motion amplitudes due to the data set having been collected over many runs with cathodes at various stages of erosion.

\subsection{Cathode processes affecting arc stability}

In the previous section the cathode spot motion model of the arc instability was developed and applied to the experimental data on the arc column displace- 


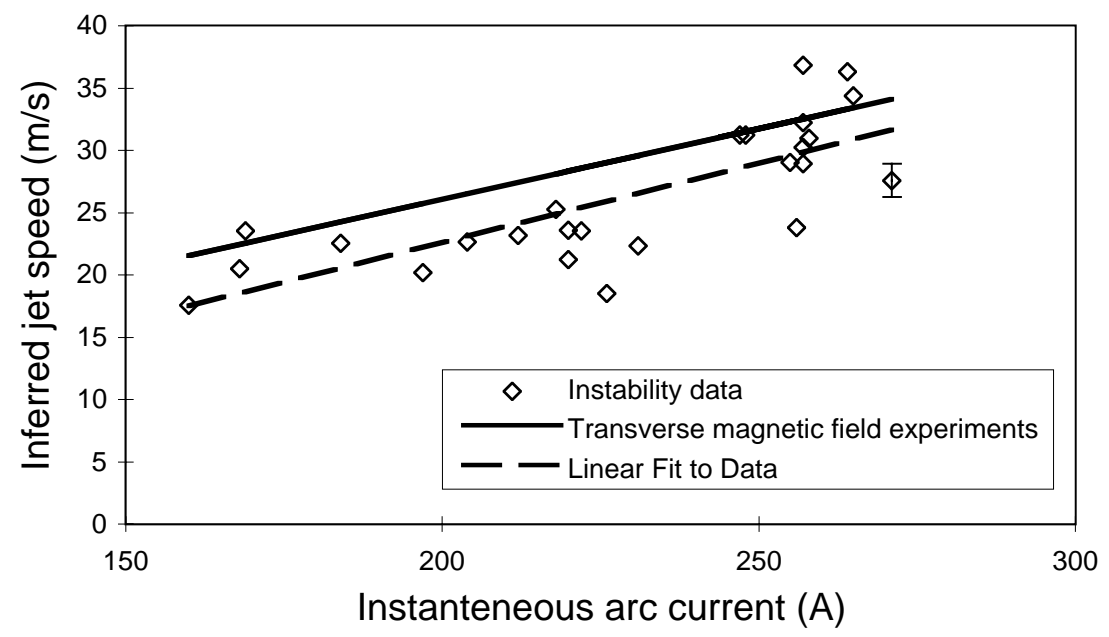

Figure 4.27: The jet speed inferred from the fits of the model to unstable arc shape as compared with the jet speed inferred from the transverse magnetic field experiments (solid line, from Figure 3.17) for instantaneous arc current. A least squares fit to the instability data is also shown. The error bars are typical standard errors from the fits.

ment. This section discusses the processes in the cathode region of the arc, the cathode surface, and the cathode bulk which could be responsible for the motion of the cathode spot itself.

Motion of cathode spots in vacuum arcs and arcs on non-refractory, or "cold" cathodes has been has been extensively discussed in the literature (see, for example [57], [58], and [59] and references therein). However, no comprehensive theory of their spot motion exists. Spot motion on the refractory cathodes, such as carbon, has received less attention and is also not understood [2]. This is probably due to the fact that in the low current arcs, which were studied the most from the basic physics point of view, the cathode spot is fixed or slowly moving [8].

The remainder of this section is organized as follows: Section 4.4.1 discusses the conditions under which the cathode spot may be mobile on refractory cathodes; Section 4.4.2 discusses possible reasons for the spot displacement. However, a definitive quantitative explanation for the observed spot motion has not been found. A discussion of the unresolved issues in identifying the causes of this cathode spot motion is given in Section 4.4.2. 


\subsubsection{Cathode temperature distribution influence on spot mobility}

As discussed in Section 4.2.1, the cathode geometry, and cathode tip diameter in particular, has a direct effect on arc stability. This section connects this observation to the cathode spot motion model of instability by considering first how the temperature distribution on the cathode surface can affect the mobility of the spot on the cathode, and then the dependence of the temperature distribution on the cathode geometry.

The electron emission on graphite cathodes in a high pressure arc for arc currents $(\lesssim 1 \mathrm{kA})$ are governed by thermionic emission, with the saturation electron current density given by the Richardson-Dushman equation with the Schottky correction [8]:

$$
J_{e}=A T_{c}^{2} \exp \left(-\frac{e \phi-\sqrt{e^{3} E / 4 \pi \epsilon_{0}}}{k T_{c}}\right)
$$

where $\phi \approx 4.4 \mathrm{eV}$ is the work function for graphite, $A \approx 6 \times 10^{5} \mathrm{~A} / \mathrm{m}^{2} \mathrm{~K}^{2}, T_{c}$ is the temperature of the emitting surface, $k$ is the Boltzmann constant, $e$ is the electron charge, $E \sim 10^{7} \mathrm{~V} / \mathrm{m}$ is the electric field at the cathode surface, and $\epsilon_{0}$ is the free space permittivity. Sufficient electron emission can thus only occur for high surface temperatures. For graphite cathodes, $T_{c} \approx 4,000 \mathrm{~K}$ (sublimation temperature) with the current density $J \sim 4 \times 10^{4} \mathrm{~A} / \mathrm{cm}^{2}$.

The temperature distribution in the cathode is determined by the net power influx due to ion bombardment, conduction and radiation from the arc plasma, the joule heating due to the current distribution in the cathode, and heat sinking by conduction into the cathode bulk, radiation from the cathode surface, and heat exchange with the surrounding atmosphere. The latter is neglected in the following because the thermal conductivity of air $(0.07 \mathrm{~W} / \mathrm{m} \cdot \mathrm{K}$ at $1,000 \mathrm{~K}$ [41]) is much smaller than that of graphite $(\sim 30 \mathrm{~W} / \mathrm{m} \cdot \mathrm{K}$ at $3,000 \mathrm{~K}[60])$. Since the temperature at the cathode spot must remain at $\approx 4000 \mathrm{~K}$, an increase in the heat removal rate from the cathode results in a more peaked, high-gradient temperature distribution. In particular, the rate of heat removal is higher for larger cathode diameter due 


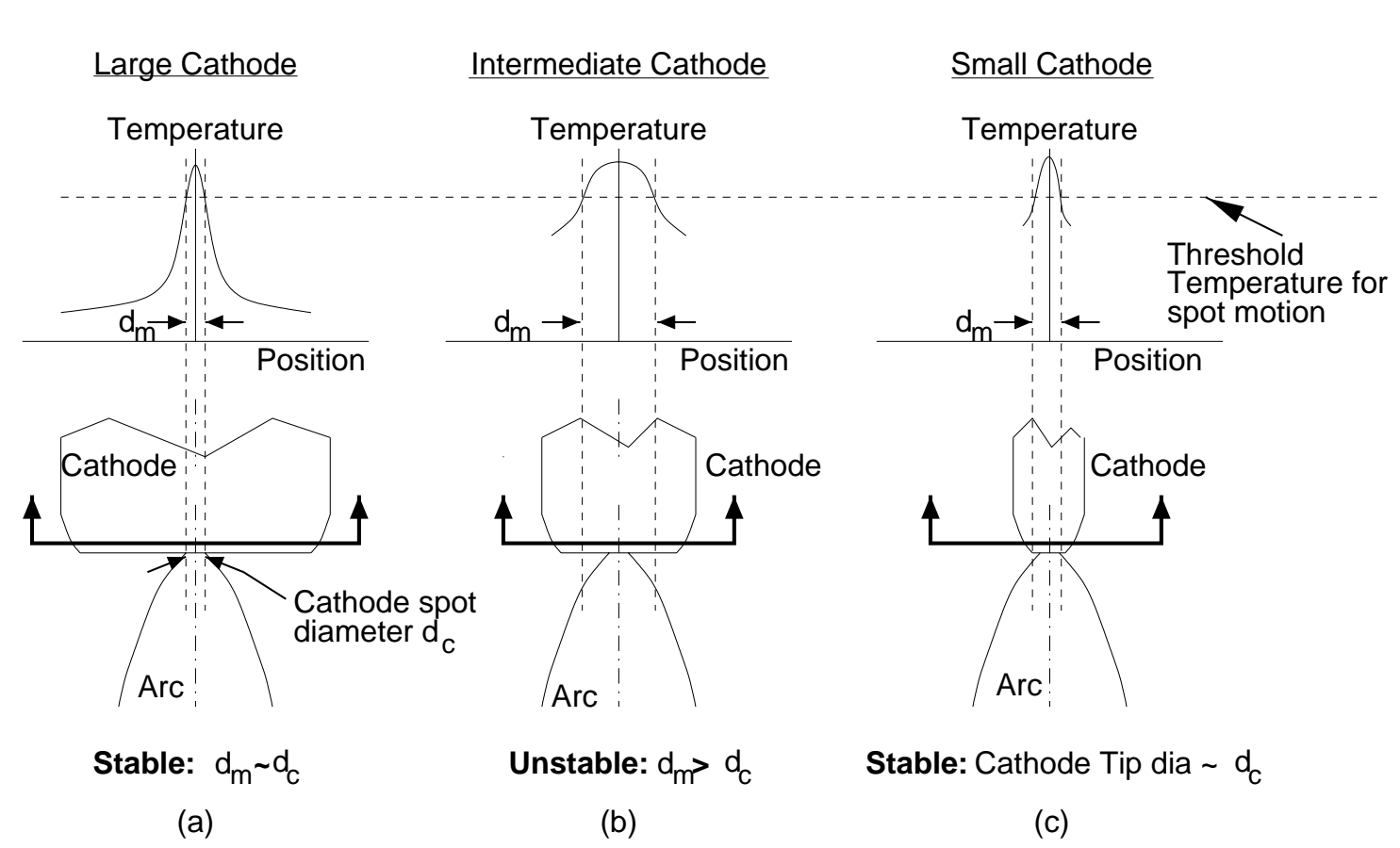

Figure 4.28: A schematic illustrating the dependence of the temperature distribution and spot mobility on the end-face of the cathode on the cathode diameter. $d_{m}$ is the diameter of the region on the cathode surface with sufficient temperature for spot motion; $d_{c}$ is the cathode spot diameter. (a) narrow temperature distribution for a large diameter cathode: $d_{m} \approx d_{c}$ and the spot is immobile; (b) a wider distribution on a smaller diameter cathode: $d_{m}>d_{c}$ and the spot can move; (c) cathode tip diameter $\approx d_{c}$ : the spot is immobile.

to increased heat conduction. This is illustrated schematically in Figure 4.28(a). Thus, it becomes less likely for the cathode spot to move to a neighboring location for a larger diameter cathode.

For sufficiently small diameter cathodes, on the other hand, the conduction of heat is replaced by radiation from the surface as the dominant mechanism of heat removal outside of the cathode spot. In this case, the temperature distribution on the tip of the cathode is more uniform, allowing for higher cathode spot mobility (Figure 4.28(b)). If the cathode tip diameter is decreased further until it is on the order of the cathode spot diameter, the spot is once again rendered immobile (Figure 4.28(c)). 
This is a qualitative explanation for the sensitivity of the arc instability to cathode tip area shown in Figure 4.14. In order to quantify this discussion, the cathode radius at which the power conducted into the cathode bulk equals that radiated from the surface can be roughly estimated as follows. The conduction is given by:

$$
P_{\text {cond }}=\frac{\kappa \Delta T A}{L}
$$

where $\kappa$ is thermal conductivity and $\Delta T / L$ is the temperature gradient. Letting $r$ be the cathode tip radius, $A \sim \pi r^{2}, L \sim r$. Since the average cathode tip temperature $T$ is much higher than that in the cathode bulk, $\Delta T \sim T$. The conducted power then becomes:

$$
P_{\text {cond }} \sim \kappa T \pi r
$$

The radiated power, assuming black body radiation, is given by:

$$
P_{\text {rad }}=\left(\text { Surface Area) } \sigma_{s} T^{4} \sim 3 \pi r^{2} \sigma_{s} T^{4}\right.
$$

where $\sigma_{s}$ is the Stefan-Boltzmann constant. Equating (4.20) and (4.21) and solving for $r$ obtain

$$
r \sim \frac{\kappa}{3 \sigma_{s} T^{3}} \sim \frac{(30 \mathrm{~W} / \mathrm{m} \cdot \mathrm{K})}{3\left(5.7 \times 10^{-8} \mathrm{~W} / \mathrm{m}^{2} \mathrm{~K}^{4}\right)\left(3 \times 10^{3} \mathrm{~K}\right)^{3}} \sim 6 \times 10^{-3} \mathrm{~m}=6 \mathrm{~mm}
$$

where the average tip temperature $T$ was taken to be $3,000 \mathrm{~K}$ and the value for $\kappa$ at 3,000 K was taken from Ref. 60 .

For radii smaller than the above estimate, the conducted power decreases, while power radiated increases due to increase in temperature, assuming the input power remains the same. Thus, at half the radius $(r=3 \mathrm{~mm})$ the conducted power decreases by a factor of 2 while the radiated power increases by a factor of $3 / 2$ via a temperature increase to $3,400 \mathrm{~K}$.

In the above, $\kappa$ was assumed constant. In fact, thermal conductivity of graphite falls rapidly above $3,000 \mathrm{~K}$, decreasing from approximately $30 \mathrm{~W} / \mathrm{m} \cdot \mathrm{K}$ 
at $3,000 \mathrm{~K}$ to $5 \mathrm{~W} / \mathrm{m} \cdot \mathrm{K}$ at $3,800 \mathrm{~K}[60]$, and making $P_{\text {rad }} \gg P_{\text {cond }}$ and therefore requiring higher average tip temperature.

Arc current is also a factor in determining the temperature distribution in the cathode. The heat flux into the cathode has been found empirically to increases linearly with arc current (for a tungsten cathode and up to $1 \mathrm{kA}$ current [61]). This leads to an increase in the average surface temperature of the tip, increasing the spot mobility.

From the above reasoning a relationship is suggested to the instability observations, in which the arc is unstable only for a range of cathode tip areas (cylindrical cathode type in Figure 4.14), and for arc currents above 120 A. The upper threshold on cathode area for instability corresponds to cathode tip radius of approximately $3 \mathrm{~mm}$, about where the heat removal becomes radiation dominant and the tip temperature is high enough to allow spot mobility. Sufficient current is also required to maintain a high temperature on the cathode surface surrounding the spot. An example of the effects of the cathode diameter and arc current on the cathode surface temperature distribution is shown in Figure 4.29. As can be seen in the figure, the bright area on the cathode is larger in the higher current case (b) and almost non-existent outside the cathode spot for the large diameter cathode (c).

Additional support for the hypothesis of cathode temperature distribution affecting stability comes from the "necked" cathode run described above in Section 4.2.2. The arc was unstable while it was attached to the wide middle section of the cathode, even though the area of that section exceeded by approximately a factor of 2 the upper threshold area for instability found for solid cylindrical cathodes. In this case, the cathode tip had high thermal resistance despite the large area because the connection to the cathode bulk was only through the narrow $(<4.6 \mathrm{~mm}$ dia.) upper neck. The heat conduction through the neck can be estimated as follows:

$$
P_{\text {conduct }}=\frac{\kappa \Delta T A}{L} \approx 120 \mathrm{~W}
$$




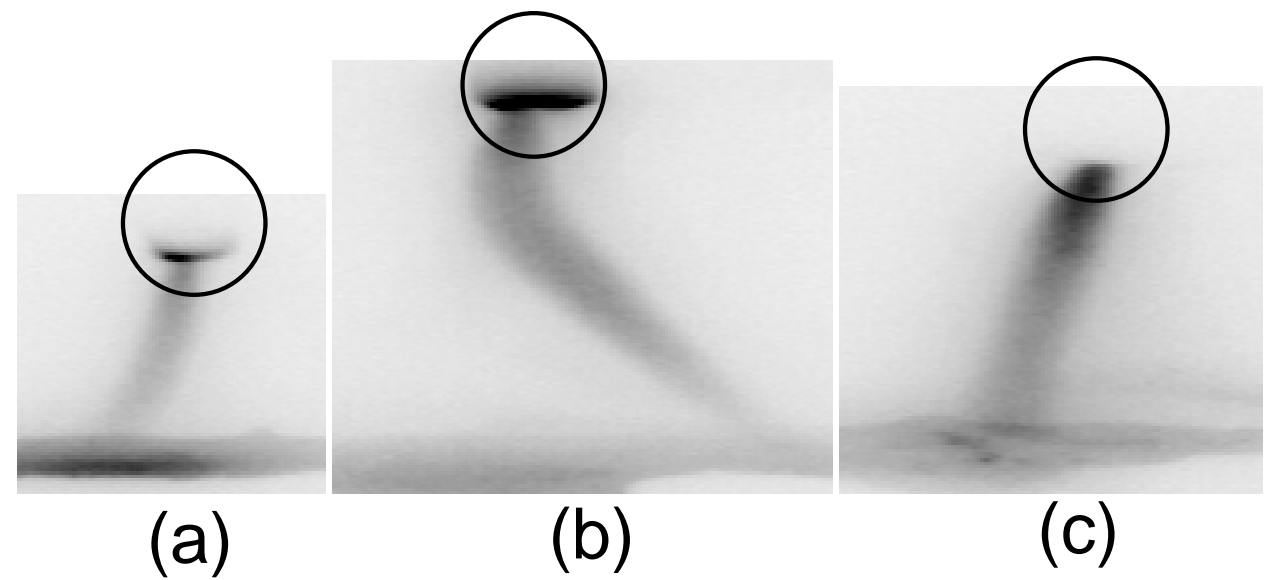

Figure 4.29: The brightly glowing high temperature area on the cathode increases for higher arc current and decreases for larger tip diameter (images are negatives, cathode is circled): (a) 3/8" diameter cathode at $113 \mathrm{~A}$; (b) $3 / 8^{\prime \prime}$ diameter cathode at $240 \mathrm{~A}$; (c) 1" diameter cathode at $253 \mathrm{~A}$ (the cathode is too dim to be visible). The arc is stable in (a) and (c) and unstable in (b). The magnification and exposure time are the same for all images; camera gain is the same for (b) and (c) and is $1.5 \times$ higher for $(\mathrm{a})$.

where $\kappa=10 \mathrm{~W} / \mathrm{m} \cdot \mathrm{K}$ is the heat conductivity at $3,500 \mathrm{~K}[60], \Delta T=3,000 \mathrm{~K}$ is the temperature difference across the neck, $A=1.6 \times 10^{-5} \mathrm{~m}^{2}$ is the cross-sectional area, and $L=4 \times 10^{-3} \mathrm{~m}$ is the length of the neck.

Such low heat conduction (compared to the power entering the cathode through the cathode spot) would imply that heat removal from the tip occurred mostly by radiation and evaporation, which would only be significant around 4,000 K. Thus, the "necked" cathode run, in addition to demonstrating instability dependence on cathode diameter, showed also that the arc can be unstable on a tip of larger diameter as long as the temperature of the tip can be maintained at a sufficiently high level.

The occurrence of instability for cathode tip areas higher than the range for solid cylindrical cathodes, such as drilled and "paddle" shaped (Figure 4.14) is probably a result of the cross-section of the cathode away from the tip itself being smaller than the cross section of a solid cylindrical cathode, which increases away from the tip. Thus, for example, while the area of the tip of the standard 
$3 / 8^{\prime \prime}$ electrode is $\approx 25 \mathrm{~mm}^{2}$, its cross-section is $71 \mathrm{~mm}^{2}$ in the bulk, whereas the standard "paddle" cathode (\# 16 in Table 4.1) has $48 \mathrm{~mm}^{2}$ cross-section. The actual geometry of the tip (solid cylindrical vs. drilled vs. rectangular) probably also plays a role in the heat conduction from the area surrounding the spot, affecting the thresholds for instability. Additionally, in the case of the drilled cathodes, the arc was attached near the center of the tip, and some of the time possibly on the inside of the hole, increasing the heat flux to the cathode from the arc column.

The heat flux from the arc column would also be higher if the cathode tip has a crater in it (is concave), since more heat would be incident on the cathode surface by radiation and conduction from the arc plasma. A crater on the tip was often observed at the end of runs with the standard cathode (Figure 4.13(e)).

An irreversible factor in determining the temperature of the tip is that if the cathode spot begins to move rapidly, the effective area under the spot through which heat is conducted into the cathode increases. The heat flux into the tip thus increases, allowing for greater spot mobility. This could account for the observation of the instability amplitude increasing over time once the instability sets in. The higher heat flux could also be responsible for the $\approx 20 \%$ higher cathode erosion rate seen during instability (Section 4.2.4).

\subsubsection{Possible reasons for spot motion}

The above discussion gave an indication of when the cathode spot can become mobile on the cathode, i.e. the spot can only move on a surface of sufficiently high temperature.. This section gives possible mechanisms for the spot motion within this region. The mechanisms considered are summarized in Table 4.4 and are discussed below. As can be seen from the table, the frequencies expected from current distribution effects in the cathode and cathode vaporization are on the order of those observed experimentally. A rigorous quantitative calculation of the stability of the arc attachment spot at the cathode requires the solution of the full set of time-dependent equations for the energy and particle balance at the cathodearc interface, as well as for the current and temperature profiles in the cathode, the cathode sheath, and the adjoining plasma. Because processes occurring in the 


\begin{tabular}{|c|c|c|c|}
\hline & Description & Mechanism & Frequency \\
\hline 1) & $\begin{array}{l}\text { Resistive Heating } \\
\text { in the Cathode } \\
\text { Bulk }\end{array}$ & $\begin{array}{l}\text { - ohmic heating under the cathode spot } \\
\text { - resistivity increases with } \mathrm{T} \\
\text { - higher voltage in cathode } \\
\Rightarrow \text { spot shifts to new location }\end{array}$ & $\sim 0.1 \mathrm{~Hz}$ \\
\hline 2) & Surface Heating & $\begin{array}{l}-\sim 1 \mathrm{~kW} \text { heat flux to the cathode } \\
\text { surface } \\
\text { - temperature and resistivity rises } \\
\text { under cathode spot } \\
\Rightarrow \text { spot shifts to new location }\end{array}$ & $\sim 0.1 \mathrm{~Hz}$ \\
\hline 3) & Cathode Erosion & $\begin{array}{l}\text { - erosion creates a "step" on cathode } \\
\text { surface } \\
\Rightarrow \text { unbalanced entrainment of gas }\end{array}$ & $\sim 0.1 \mathrm{~Hz}$ \\
\hline 4) & $\begin{array}{l}\text { Current Distribution } \\
\text { in the Cathode }\end{array}$ & $\begin{array}{l}\text { 1. Current is constricted in the } \\
\text { cathode and in the arc } \\
\Rightarrow \text { a repulsive force } \\
\Rightarrow \text { unstable equilibrium } \\
\text { 2. A force tending to restore the arc } \\
\text { current to the electrode axis. }\end{array}$ & $\sim 600 \mathrm{~Hz}$ \\
\hline 5) & $\begin{array}{l}\text { Vaporization from } \\
\text { the Cathode Spot }\end{array}$ & $\begin{array}{l}-\sim 1 \mathrm{~kW} \text { heat flux to the cathode } \\
\text { surface } \\
\text { - rapid surface temperature rise leads } \\
\text { to vapor production } \\
\Rightarrow \text { cold, resistive vapor displaces the } \\
\text { cathode spot }\end{array}$ & $\sim 500 \mathrm{~Hz}$ \\
\hline
\end{tabular}

Table 4.4: Possible mechanisms of cathode spot motion. An order of magnitude estimate of the expected frequency of instability for each mechanism is given. Only the last two mechanisms give the expected frequency on the order of the measured one $(200-800 \mathrm{~Hz})$. The mechanisms are listed in the order they are discussed in the text.

region, such as electron emission, ion production, cathode heating and vaporization are all interdependent [62], solutions to these equations have only been obtained in the literature numerically for steady-state while treating the cathode surface as the boundary of the solution region (e.g. Ref. 51,63,64). In what follows, only orders of magnitude estimates of individual physical mechanisms are attempted.

\section{Resistive heating in the cathode bulk}

The electrical resistivity of graphite for temperatures above $\approx 1,500 \mathrm{~K}$ increases linearly with temperature $[60,65]$, and can be approximated as:

$$
\eta(T)=c_{0}\left(T+T_{0}\right)
$$

where $c_{0}=2 \times 10^{-9} \Omega \mathrm{m} / \mathrm{K}$ and $T_{0}=2,000 \mathrm{~K}[60]$. 
Above a certain critical current density, thermal runaway due to Joule heating in the cathode can take place [66], causing an increase in the voltage drop in the cathode and potentially causing a spot displacement. The magnitude of this critical current density is given for a semi-infinite cathode in Ref. 67 as:

$$
J_{\text {crit }}=\frac{2}{a} \sqrt{\kappa \sigma_{0}}
$$

where $a \approx 1.3 \mathrm{~mm}$ is the cathode spot radius for $I=250 \mathrm{~A}^{1}, \kappa$ is the thermal conductivity, and $\sigma_{0}$ is a coefficient of temperature dependence of electrical conductivity in the relation $\sigma(T)=\sigma_{0} /\left(T+T_{0}\right)$. From Eq. (4.24), $\sigma_{0}=1 / c_{0}=5 \times 10^{8} \Omega^{-1} \mathrm{~m}^{-1} \mathrm{~K}$. From Ref. $60, \kappa=5 \mathrm{~W} / \mathrm{m} \cdot \mathrm{K}$ at $3,800 \mathrm{~K}$. The critical current for thermal runaway is then

$$
I_{\text {crit }}=\pi a^{2} J_{\text {crit }} \approx 400 \mathrm{~A}
$$

This critical current is approximately a factor of 2 higher than the range in the present experiments (150-250 A). However, the above estimates were done for a semi-infinite cathode and the critical current should be lower for a finite cathode radius ( $\sim 2-3 \times$ the cathode spot radius) since heat conduction would be smaller and the joule heating higher. It is thus plausible that there is a significant voltage increase due to joule heating in the cathode under the cathode spot.

However, the time scale for the development of the thermal runaway condition is [67]:

$$
\tau \sim \frac{\rho c_{p} \pi^{2} a^{4}}{c_{0} I^{2}} \sim 0.8 \mathrm{sec}
$$

where $a \approx 1.3 \mathrm{~mm}$ is the cathode spot radius for $I=250 \mathrm{~A}$ [68], the graphite mass density $\rho=1.8 \times 10^{3} \mathrm{~kg} / \mathrm{m}^{3}$ and heat capacity $c_{p}=2 \times 10^{3} \mathrm{~J} / \mathrm{kg} \cdot \mathrm{K}$ are taken from Ref. 60, and $c_{0}$ is defined by Eq. (4.24). This time scale is much slower than that of the instability time scale $\left(\sim 2 \times 10^{-3} \mathrm{sec}\right)$, and therefore thermal runaway cannot account for the instability.

\footnotetext{
${ }^{1}$ Assumed from the current density value of $4,500 \mathrm{~A} / \mathrm{cm}^{2}$ at $250 \mathrm{~A}$ [68].
} 


\section{Surface heating}

The intense cathode surface heating in the cathode spot also heats the region underneath the cathode spot, increasing resistivity and potentially causing a spot displacement. The time scale for the increase to propagate to depth on the order of the cathode spot radius $a$ is [69]:

$$
\tau_{\text {th }} \sim \frac{a^{2}}{\delta} \approx 1.3 \mathrm{sec}
$$

where thermal diffusivity $\delta=\kappa / c_{p} \rho \approx 1.3 \times 10^{-6} \mathrm{~m}^{2} / \mathrm{s}$ is taken from Ref. 60. As with resistive heating, this time scale is much slower than that of the instability time scale.

Both the resistive and surface heating, however, are on the time scale of the slow precession of the cathode spot observed for a stable arc, mentioned in Section 3.2.1. According to Eq. (4.28), the spot would move a spot diameter in $1.3 \mathrm{sec}$, completing one revolution along the periphery of a $6 \mathrm{~mm}$ diameter cathode tip in $\sim 10 \mathrm{sec}$. The observed precession period is approximately $20 \mathrm{sec}$. Thermal time scales, therefore, while appearing too slow for the instability, could account for the observation of the slow motion of the cathode spot.

\section{Cathode erosion}

The cathode erosion rates where given in Section 4.2.4 as approximately $0.06 \mathrm{~mm} / \mathrm{s}$ for approximately $5 \mathrm{~mm}$ tip diameter. For an instability frequency of $500 \mathrm{~Hz}$, this would imply an erosion rate per period of oscillation of

$$
\frac{0.06 \mathrm{~mm} / \mathrm{s}}{500 \mathrm{~Hz}}=1.2 \times 10^{-4} \mathrm{~mm}
$$

Since the cathode sheath layer length is on the order of $\lambda_{m f p} \sim 10^{-3} \mathrm{~mm}$ [Eq. (4.32)], cathode erosion is too slow to affect spot motion on the instability time scale.

The time scale of the slow precession of the spot on the cathode, however, with a period of $\sim 20 \mathrm{~s}$, implies an erosion of $\sim 1 \mathrm{~mm}$ of cathode material per pass. This can create a geometry with asymmetric entrainment of surrounding 


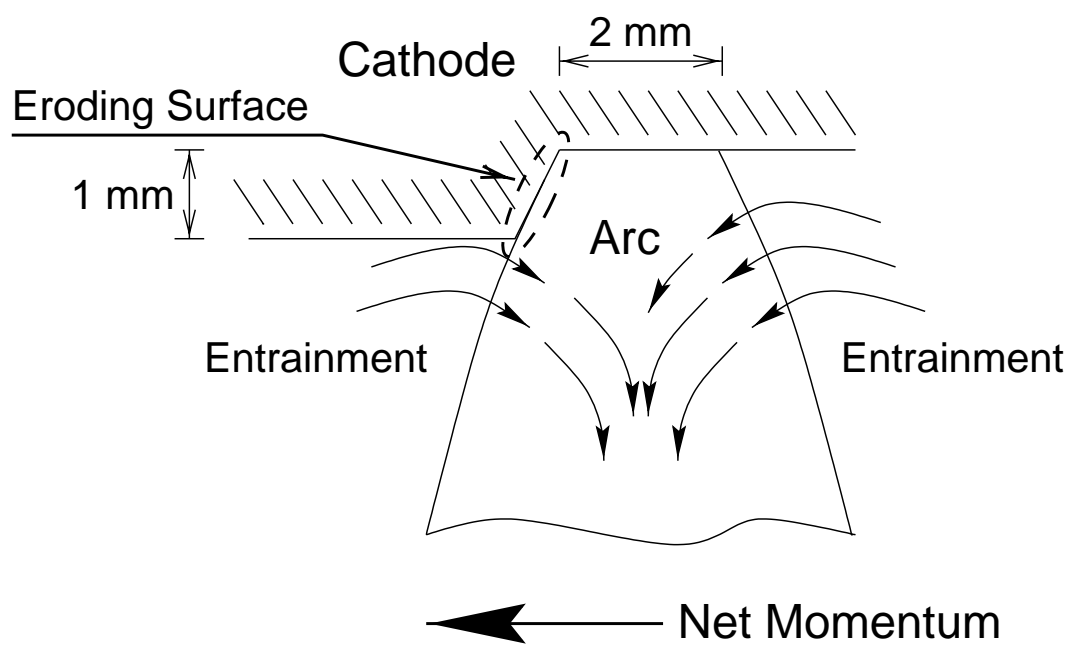

Figure 4.30: Schematic illustrating a possible spot motion mechanism due to asymmetric entrainment of gas. The edge of the eroding surface (circled) is causing less gas to be entrained on that side, creating a momentum imbalance.

gas into the jet, providing the transverse momentum for spot motion, as shown schematically in Figure 4.30. Thus, cathode erosion, in addition to the thermal runaway discussed above, is also a possible mechanism of the observed slow motion of the spot on the cathode.

\section{Interaction with the current distribution in the cathode}

The resistive diffusion time scale for the graphite cathode is:

$$
\tau \sim \frac{\mu_{0} L^{2}}{\eta} \sim \frac{\left(4 \pi \times 10^{-7} \mathrm{H} / \mathrm{m}\right)\left(5 \times 10^{-3} \mathrm{~m}\right)^{2}}{1 \times 10^{-5} \Omega \cdot \mathrm{m}} \sim 3 \times 10^{-6} \mathrm{~s}
$$

where the length scale $L$ was taken to be the radius of the standard $3 / 8^{\prime \prime}$ cathode. Since the time scale of the instability is $\sim 10^{-3} \mathrm{~s}$, the resistive diffusion time is much faster then the time scale of the instability. Resistive diffusion thus does not play a role in the mechanism of the instability and the current distribution in the cathode can be assumed to change instantaneously as the spot is moving on the cathode. Resistivity of the arc plasma $\left(1 \times 10^{-4} \Omega \cdot \mathrm{m}[70]\right)$ is higher than that of the graphite cathode, implying an even shorter resistive diffusion time in the arc plasma and no role in the instability mechanism. 


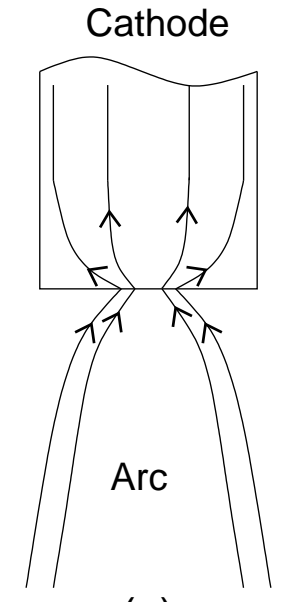

(a)

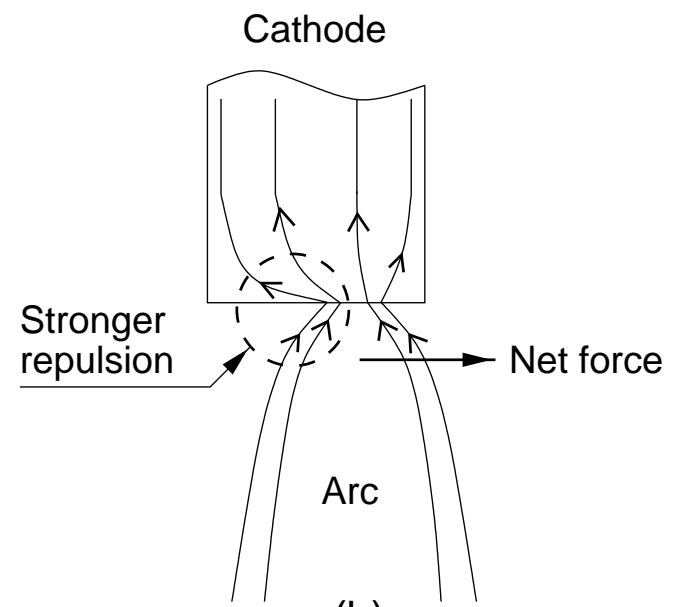

(b)

Figure 4.31: A schematic representing approximate current lines in the cathode and the cathode region of the arc for (a) cathode spot centered on the cathode and (b) displaced to the side.

Since the diameter of the cathode tip is normally larger than that of the cathode spot, a current constriction exists in the cathode tip, as depicted schematically in Figure 4.31(a). The extent of the constriction into the cathode bulk is on the order of the cathode radius [71]. Since the arc is also constricted in the region of the cathode spot, a mutual repulsion force acts between the two current distributions. When the spot is centered on the cathode, this force has no unbalanced component parallel to the cathode surface. This equilibrium point is unstable, however, and a small displacement of the spot to either side of the center results in an unbalanced component of the force acting in the direction of the displacement (Figure 4.31(b)). Thus, a stable equilibrium point for the cathode spot is likely to be off-center on the cathode. While not providing for continuous spot motion, this effect could be part of the spot motion mechanism.

On the other hand, the current in the cathode bulk tends to restore the arc current to the electrode axis. Witkowski [33] estimated a frequency of oscillation of the arc column on the basis of the interaction between the currents in the arc column and in the cathode. In his work it was assumed that the arc is attached at a fixed point on the cathode and that it is the angle the arc column makes with the cathode that is changing (see Figure 4.32). By assuming further straight line 


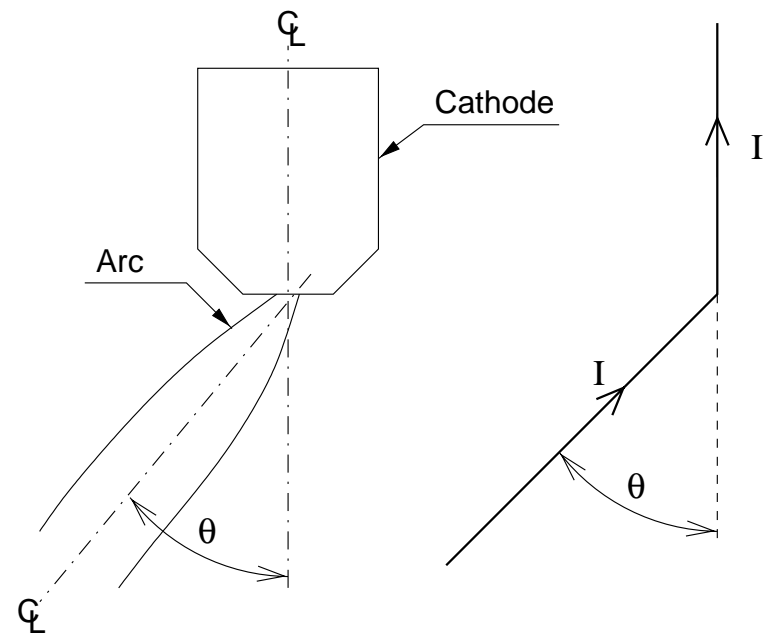

Figure 4.32: Schematic illustrating the assumption of Witkowski [33] in deriving the frequency of arc oscillation.

current distributions, a force was computed between the current in the electrode and the current in the column adjacent to it that tends to restore the arc column to vertical position ( $\theta=0$ in the figure). By balancing this restoring force and the moment of inertia of the arc column about the cathode attachment point, a harmonic oscillator type differential equation for the angle was obtained, with the natural frequency given by:

$$
f=\frac{I}{2 \pi L} \sqrt{\frac{3 \mu_{0}}{8 \pi \rho S}}
$$

where $L$ is an equivalent length of the straight section of the arc column near the cathode, $\rho$ is mass density, and $S$ is the area of arc cross-section. Assuming $L=1 \mathrm{~cm}, \rho S=m=6 \times 10^{-6} \mathrm{~kg} / \mathrm{m}$, where $m$ is the linear mass density inferred from transverse magnetic field experiments, and for $I=250 \mathrm{~A}$, get $f=630 \mathrm{~Hz}$, which is in approximate agreement with observed instability frequencies (see, for example, Figure 4.4).

The assumption of a fixed cathode spot and varying angle does not seem to be justified by observations in the present experiments. The cathode spot is mobile, and although the angle the column makes with the axis of the cathode can vary as in the case in Figure 4.16, the column is approximately normal to the cathode surface. 
Nonetheless, the agreement of the above estimate of oscillation frequency with the measured frequency of the instability suggests that electromagnetic interaction may be part of the spot motion mechanism.

\section{Cathode vaporization.}

The high temperatures found in the cathode spot result in vaporization of cathode material [59]. Intense vaporization has been used previously to account for motion of spots on graphite anodes in the constricted anode mode [62]. Influx of the carbon vapor with temperature much lower than that of the arc plasma $(4,000 \mathrm{~K}$ vs. approximately $12,000 \mathrm{~K}$ ) would tend to lower the conductivity in the electrode region of the arc and would favor displacement of the spot to a new location. Unlike at the anode, the cathode has an ion flux impinging on its surface accelerated by the cathode fall and maintaining the cathode temperature necessary for electron emission. However, this flux would not prevent the relatively cold cathode vapor from reaching the arc column, as can be seen from the following.

The ions that constitute the flux to the cathode are produced by electron impact mainly within a single electron mean free path from the cathode surface [9]. The electron mean free path is

$$
\lambda_{m f p} \sim \frac{1}{\sigma n_{0}} \sim \frac{1}{\left(5 \times 10^{-15} \mathrm{~cm}^{2}\right)\left(2 \times 10^{18} \mathrm{~cm}^{-3}\right)} \sim 10^{-4} \mathrm{~cm}
$$

where $\sigma$ is a characteristic cross section, weakly dependent on temperature, for collisions of electrons with neutrals [39], and $n_{0}$ is the neutral density at $4,000 \mathrm{~K}$ assuming equilibration with atmospheric pressure. The transit time of vapor atoms across $\lambda_{m f p}$ is

$$
\tau \sim \frac{\lambda_{m f p}}{v_{t h}} \sim \lambda_{m f p} \sqrt{\frac{m}{k T}} \sim \frac{10^{-4} \mathrm{~cm}}{1.7 \times 10^{5} \mathrm{~cm} / \mathrm{s}} \sim 6 \times 10^{-10} \mathrm{~s}
$$


The collision frequency of the vapor atom with the ion flux is

$$
\begin{aligned}
\nu \sim(\text { Flux })(\sigma) \sim\left(\frac{J_{\text {ion }}}{e}\right) \sigma \sim & \left(\frac{0.1 J_{e}}{e}\right) \sigma \sim \\
& \left(\frac{450 \mathrm{~A} / \mathrm{cm}^{2}}{1.6 \times 10^{-19} \mathrm{C}}\right)\left(10^{-14} \mathrm{~cm}^{2}\right) \sim 3 \times 10^{7} \mathrm{~s}^{-1}
\end{aligned}
$$

where the ion fraction of the current to the cathode is taken from Ref. 9 to be $\sim 0.1$ and the current density at the cathode is taken to be 4,500 A/cm ${ }^{2}$ [68]. The number of collisions a vapor atom would undergo while in the sheath is

$$
\tau \nu \sim 2 \times 10^{-3} \text { collisions }
$$

Thus the vapor would move through the sheath before suffering collisions with the returning ions and therefore could affect the conductivity in the pre-sheath plasma.

Experimental evidence for this effect comes from spectroscopic investigations with a $10 \mathrm{kA}$ copper cathode arc, were a higher temperature plasma was found to rotate around the copper vapor jet [72]. A spectroscopic and photographic studies by Mentel [73] find a cold (4,000-5000 K) cathode vapor jet persistent for over $1 \mathrm{~cm}$ from the cathode surface for the case of a $1 \mathrm{kA}$ carbon arc. The vapor jet is enveloped by the current-carrying plasma and is concentric with it in the case of a stationary cathode spot and is asymmetric w.r.t the current-carrying plasma for a non-stationary cathode spot. It is furthermore suggested in the paper that the asymmetric current distribution created by the vapor jet in the latter case generates magnetic forces which tend to displace the cathode spot from the vapor production site.

The frequency of spot rotation on the cathode based on this effect can be estimated as follows. The time scale for the cathode surface to reach sublimation temperature at a new cathode spot location and for vapor production to begin is $[69, \mathrm{p} .72]$ :

$$
\tau \sim \frac{\kappa^{2}(\Delta T)^{2}}{q^{2} \delta} \sim 5 \times 10^{-4} \mathrm{sec}
$$


where $\kappa \sim 10 \mathrm{~W} / \mathrm{m} \cdot \mathrm{K}$ and $\delta \sim 1.3 \times 10^{-6} \mathrm{~m}^{2} / \mathrm{s}$ as above, $\Delta T \sim 500 \mathrm{~K}$, and $q \sim 2 \times 10^{8} \mathrm{~W} / \mathrm{m}^{2}$ is the energy flux on the cathode surface within the cathode spot diameter. If the vapor production time $\tau$ is the limiting time scale for the spot displacement, the $2.6 \mathrm{~mm}$ diameter cathode spot would complete a revolution around the $6 \mathrm{~mm}$ diameter cathode tip in $\sim 1.8 \times 10^{-3} \mathrm{sec}$, implying a frequency of $\sim 550 \mathrm{~Hz}$, similar to the observed frequencies.

\subsection{Industrial Application}

In this section, an extrapolation of the results of this Chapter to an industrial scale DC arc furnace will be made, as was done in Section 3.5 for arc deflection in magnetic field. As mentioned in that section, since this involves an extrapolation over almost three orders of magnitude in arc current, as well as neglect of complexities such as foamy slag, only approximate estimates can be made without a more specific study.

Assuming as in Section 3.5 an arc current of $100 \mathrm{kA}$, arc length of $0.5 \mathrm{~m}$, and jet speed of $1 \mathrm{~km} / \mathrm{s}$ [2], the frequency of oscillation of the cathode spot required to produce a significant disturbance ( $\sim$ one wavelength) on the arc column is $f \sim$ $(1000 \mathrm{~m} / \mathrm{s}) /(0.5 \mathrm{~m})=2 \mathrm{kHz}$. Measurements of sound spectra from industrial furnaces [74] show broadband noise between 100 and 2,000 Hz.

In order for the arc column to be displaced the same angle as in the present experiments $\left(\approx 10^{\circ}\right.$, Figure 4.24), $\xi \sim(0.5 \mathrm{~m}) \tan \left(10^{\circ}\right) \sim 0.1 \mathrm{~m}$ at the anode, and the cathode spot motion amplitude required is, according to Eq. (4.7),

$$
a \sim \frac{\xi v}{z \omega} \sim \frac{(0.1 \mathrm{~m})(1000 \mathrm{~m} / \mathrm{s})}{(0.5 \mathrm{~m})(2 \pi)(2000 \mathrm{~Hz})} \sim 0.016 \mathrm{~m}=1.6 \mathrm{~cm}
$$

Assuming current density in the cathode spot to be the same as observed in a $10 \mathrm{kA}$ carbon $\operatorname{arc}\left(\approx 5,000 \mathrm{~A} / \mathrm{cm}^{2}[75]\right)$, the cathode spot radius is $\sim 2.5 \mathrm{~cm}$, and the above displacement amplitude represents approximately $1 / 2$ the spot radius, similar to what was found in the present experiments. 
The above requirement of motion of $1.6 \mathrm{~cm}$ in a quarter of a $2000 \mathrm{~Hz}$ cycle gives cathode motion speed of $\sim 130 \mathrm{~m} / \mathrm{s}$. Cathode motion on the order of $100 \mathrm{~m} / \mathrm{s}$ has been observed in $10 \mathrm{kA}$ carbon arcs [75].

Thus it seems plausible that spot motion is responsible at least for some of the instabilities associated with the industrial arc furnaces. Based on Table 4.4, the most likely spot motion mechanisms are current distribution effects in the cathode and cathode vaporization. Both are likely to remain important at higher currents, because of higher current densities and higher heat fluxes to the cathode.

\subsection{Discussion and Conclusions}

\subsubsection{The cathode spot motion model and its relevancy to experimental observations}

A systematic study has been conducted of the unstable arc behavior in the 100-250 A current range. A central finding of the experiments is the strong dependence of the instability on the cathode geometry: for the same arc current, the arc is stable or unstable for different cathodes. In particular, for the cylindrical cathodes, such as used in industry, the instability occurs only for a relatively narrow range of cathode diameters (Section 4.2). The unstable behavior is explained in terms of the cathode spot motion (Section 4.3). The conditions for the spot to be mobile on the cathode are discussed and appear consistent with those seen in the experiments (Section 4.4.1).

The picture of the "instability" that thus emerges is not that of a standard type of exponentially growing instability of, for example, ideal MHD, but is rather a result of periodic motion of the cathode attachment spot on the surface of the cathode. Possible mechanisms for this spot motion were discussed in Section 4.4.2.

The spot motion model successfully explains the arc instability observations. Arc column oscillation in one plane, as, for example, in Figure 4.12, can be understood as special cases of the spot moving back-and-forth on a line on the cathode. The instability for short arc lengths, with only a fraction of a wavelength on the 
arc column, is also consistent with the transit time of the jet for short arcs being smaller then the period of oscillation of the spot.

An equation for the arc displacement during instability is derived on the basis of the spot motion model and the one-dimensional thin-wire model of the arc dynamics developed previously for the arc in transverse magnetic field. The shape of arc in the majority of low to moderate amplitude instability cases is well fitted by the model. Measured values of the cathode spot motion amplitude are in approximate agreement with the values of the spot motion amplitude required by the model to describe the arc shape. The jet speed required by the model is within approximately $20 \%$ of the jet speed inferred on the basis of the model from the transverse magnetic field experiments and shows approximately the same rate of change with the arc current.

As shown in Section 4.3.3, the model predicts arc length variation at twice the oscillation frequency, and thus harmonic generation on the arc voltage. Depending on whether the spot is moving in a circular or more elongated orbit on the cathode, the amplitude of length variation will change. As discussed in Section 4.2.1, a harmonic of the oscillation frequency is usually present the voltage and current waveforms whose strength w.r.t. the fundamental varies over time.

The presence of the fundamental frequency on the arc voltage is not explained by the model as derived above. However, to simplify the derivation it was assumed that the cathode is flat and the spot motion is strictly in a plane parallel to the anode, which is usually not the case in the experiment because of to cathode erosion. The presence of the fundamental can, at least in part, be explained by the variation of the arc length due to this out-of-plane motion of the spot.

The influence of the self magnetic field on the arc column remains an open question. Since the arc shape during low amplitude instability is similar to that resulting from the applied transverse magnetic field, it is expected that the self-field effects would not be dominant in this parameter regime, as discussed in Chapter 3. However, the self-field probably does play a role in the case of large curvature column displacements. For example, although the large displacement of the column in Figure 4.16 can be explained qualitatively by the large amplitude spot motion 
on the curved, wide cathode tip, the self-magnetic field probably plays some role in accentuating the kinking.

The cases of large amplitude instability when there is a broadband spectrum of oscillation have not been analyzed quantitatively. It is plausible that these cases do not lie outside the framework of the spot motion model, but require a more sophisticated treatment then the one-dimensional approximation used and possible need for inclusion of self-magnetic field effects.

An additional factor that could affect the evolution of the column shape is the possible variation of the jet speed during the transit time of the jet across the gap from the cathode to the anode. Since the frequency of the column motion is present on the arc current, and since the jet speed is proportional to current, the jet speed is varying with the frequency of the instability. If, furthermore, the frequency is commensurate with the transit time, this would imply a variation of the jet speed along the arc length, which is not included in the model, and could account for cases such as shown in Figure 4.17(c) which are not well-fitted by the model. This could also be a source of the spread in the values of the inferred jet speeds. In addition, a significant lag time in full development of the jet after a current variation could account for the values inferred from instability fits being approximately $20 \%$ below those inferred from the transverse magnetic field experiments.

The occurrence of instability for only a range of cathode diameters is interpreted using the cathode spot motion model as follows. It is argued that due to the temperature dependence of the thermionic emission mechanism, the spot mobility increases for higher and more uniform cathode temperatures. Furthermore, the cathode temperature and temperature uniformity are shown to be higher for smaller cathode diameters. Thus, if the instability is caused by the motion of the spot on the cathode, it will occur for diameters small enough for the spot to be mobile. This diameter is shown to be approximately equal to that observed.

The lower bound on the cathode diameter for instability is interpreted as a physical limit on the excursion of the cathode spot. This lower bound would thus be expected to equal the cathode spot diameter. However, the lower bound diameter is observed to be $\approx 5 \mathrm{~mm}$, still approximately twice the cathode spot diameter at $250 \mathrm{~A}(\approx 2.6 \mathrm{~mm}[68])$. This may be an indication that the mechanism driving 
the spot motion ceases to apply below this diameter, even though the spot still has room to move, or that the spot size increased to cover the cathode end-surface.

\subsubsection{Mechanism of the cathode spot motion}

The question of the physics of spot motion remains open. Several possible mechanisms of the spot motion are investigated. Two of the candidates, thermal runaway in the cathode and cathode erosion, although both potentially able to produce spot motion, are too slow for time scales of the instability. They do, however appear to be relevant for the slow precession of the cathode spot.

One of the two likely mechanisms of spot motion is a magnetic interaction of the arc constriction in the cathode region with the current distribution in the cathode. It is argued that a cathode spot centered on the axis of the cathode may not be in a stable equilibrium w.r.t. the forces between currents in the arc and in the cathode. A more quantitative assessment of this effect is needed. An estimate of the frequency of spot oscillation based on magnetic interaction under somewhat different assumptions gives the right order of magnitude for the instability.

Cathode vaporization is considered as the other likely cause of spot displacement. I have put an order-of-magnitude estimate in Section 4.4.2 that indicates that the relatively cold vapor from the cathode will enter the pre-sheath plasma unimpeded by the ion flux to the cathode. The vapor would thus lower the conductivity in the plasma, tending to displace the current to the side. There is evidence for this effect from spectroscopic experiments (Section 4.4.2).

\subsubsection{Other possible models of the instability}

The characteristics of the three most likely models of instabilities were summarized in Section 4.3.1. The applicability of the cathode spot motion model to the observations was discussed above. The other two possible candidates are a current-

driven "kink" and jet-driven "firehose" type instabilities. Either one would explain the observed growth of instability amplitude with current: the latter one because 
the jet speed increases with current. However, they are unlikely to be significant in these experiments for the following reasons.

Neither the "kink" nor the "firehose" instabilities can easily explain the observed occurrence of the instability only for a narrow range of cathode diameters. These instabilities might be triggered by a change in the current density or the jet speed in the arc column. However, if there is any dependence of these quantities on the cathode diameter, it would be expected to be monotonic, and not specific to a narrow range. Moreover, the influence of the cathode geometry on the unstable arc shape, such as seen in the examples of motion in one plane with the "paddle" shaped cathodes, is not explained by these instabilities.

The displacement of the center of mass of the arc column is observed during many unstable runs, as, for example, in Figure 4.16. It is also apparent during instability at short arc lengths, when the column is deformed only a fraction of a wavelength and moves side-to-side as a whole. A displacement of the center of mass of the column has to involve interaction with the electrodes, and cannot be due solely to a column instability like the "kink" or the "firehose", since in that case momentum conservation would be violated.

Both a "kink" and a "firehose" instability would be expected to have exponential growth in time and therefore with distance from the cathode, since the plasma is streaming away from the cathode at the jet speed. In the experiments, linear growth is observed, consistent with the cathode spot motion model. Finally, it is expected that both the "kink" and the "firehose" modes would be stable for short arcs, when the transit time of the jet across the electrode gap is shorter then the growth time of the instability, whereas the arc is unstable for all lengths studied. 


\section{Chapter 5}

\section{Conclusions and Future Work}

The research done in this thesis has furthered the knowledge of atmospheric pressure arcs in several ways. An apparatus and a set of diagnostics was established that enabled systematic measurements of arc behavior in applied transverse magnetic field and of spontaneous arc instability without an external magnetic field. It has been shown that the arc phenomena studied are determined by electrode surface and electrode jet effects, as well as by the physics of the arc column. A simple one-dimensional model has been developed for arc dynamics that appears to be suitable for the description of both the arc deflection by a transverse magnetic field and for the arc instability studied. In the case of the instability, experimental evidence is presented supporting cathode spot motion as the mechanism of the unstable behavior. When coupled with the one-dimensional model, the spot motion reproduces the unstable arc shape well.

\subsection{Arc Deflection}

\subsubsection{Conclusions}

The shape of the arc deflected by the applied transverse magnetic field was measured using a gated camera for a range of arc currents, applied magnetic field strengths, as well as for a range of applied magnetic field frequencies from DC to 
$1.6 \mathrm{kHz}$ (Chapter 3). It was concluded from the results of this study that the shape of the arc column in applied transverse magnetic field is determined by a simple balance of the $\vec{J} \times \vec{B}$ force and the inertia of the cathode jet.

The model of arc deflection derived under these assumptions is consistent with the observations for a range of frequencies, from a constant (DC) applied magnetic field to oscillating fields up to the $1.6 \mathrm{kHz}$ studied. The model produces arc shapes consistent with the data using two fit parameters, linear mass density and average cathode jet velocity. The values of the two parameters are reasonable compared with expectations. The average velocity scales linearly with the arc current, as expected from Maecker's model of jet formation, and is an order of magnitude below the estimated peak value (Section 3.4.2).

The self-magnetic field effects are found to be important for low frequency and DC deflection: without inclusion of the self-field, the inferred mass density at low frequencies is a factor of 2 higher than expected from the high frequency fits (Section 3.4.2). An attempt to include the self-field effect analytically for higher frequencies using a local approximation (Section 3.3.6) produced an arc shape inconsistent with the observations. However, the consistency of the model without the self-field effect with the experiment for the whole 100-1600 Hz frequency range indicates that self-field effects do not play a significant role in this parameter regime and that the local approximation used is inaccurate (Section 3.4.4). A numerical calculation of the self-field effect also supports this view (Appendix A).

\subsubsection{Suggestions for future work}

For future work, a two-dimensional model is needed that would include radial and axial profiles of density and velocity. This would enable to accurately predict the arc deflection amplitude for low frequency and DC applied magnetic fields without having to first obtain the mass density and jet velocity from the high-frequency fits.

A more accurate, self-consistent numerical calculation of the self-field effect should be done in the future to fully resolve the issue at high applied field frequencies. Once again, a two-dimensional calculation with a current profile may be 
required as the wavelength of the bending approaches the arc displacement amplitude and the radius of curvature of the column becomes comparable to the column radius for high applied field frequencies.

The present model of arc deflection is directly applicable to devices in materials processing, where, for example, oscillating transverse magnetic fields have been used to spread the discharge over a wider processing area [52]. In addition, $\mathrm{AC}$ transverse magnetic field could be used as a diagnostic of the average flow speed and mass density in other arcs and plasma torches where strong flows are present.

An extrapolation of the model to the industrial furnace arcs gives a deflection angle similar to what is found in practice (Section 3.5). However, because the extrapolation is over almost three orders of magnitude in arc current, an intermediate current experiment $(\sim 10 \mathrm{kA})$ is desirable to confirm the model. Additional complications of the industrial furnaces, such as foamy slag and a possibly permeable steel shell have not been taken into account.

\subsection{Arc Instability}

\subsubsection{Conclusions}

Unstable arc behavior without an applied magnetic field was measured for a range of cathode diameters, geometries, arc currents, and arc lengths (Chapter 4). The results of the experiments have demonstrated that the occurrence of the instability strongly depends on the cathode geometry: for the same arc current, the arc can be stable or unstable depending on the cathode diameter (Section 4.2.2).

Cathode spot motion is proposed as the mechanism of the instability (Section 4.3). When coupled with the one-dimensional model for arc dynamics used to describe the behavior in the applied magnetic field, the spot motion model successfully explains the shape of the arc column at low amplitudes of instability. The spot motion amplitude required is in approximate agreement with the measured amplitudes. The average cathode jet speed is within $20 \%$ of that inferred on the 
basis of the model from the transverse magnetic field experiments on a stable arc, and shows the same rate of change with the arc current, as expected.

The dependence of the instability on cathode diameter is interpreted as a result of the cathode spot being mobile only for sufficiently small diameters, when the cathode tip is sufficiently hot (Section 4.4.1). Moreover, if cathode spot motion is the mechanism of the instability, there must be a lower bound on the cathode diameter for which the arc is unstable. This lower bound is expected to be the diameter of the cathode spot itself. Such a lower bound is indeed observed (Section 4.2.2), but is approximately twice the cathode spot diameter obtained from the literature.

Several possible mechanisms of spot motion have been investigated. In particular, two possibilities, thermal runaway in the cathode and cathode erosion, are shown to be too slow for the time scales of the instability. The interaction of the current distribution in the cathode with the current in the cathode region of the arc remains as the most likely cause of spot displacement. An estimate of the frequency of spot oscillation under such an interaction gives the right order of magnitude for the instability.

\subsubsection{Suggestions for future work}

As mentioned above, the lower bound on cathode diameter for arc instability is expected to be the diameter of the cathode spot itself, but is observed to be approximately twice the cathode spot diameter, as obtained from the literature. For future work, it would thus be useful to have a direct measurement of the cathode spot size. This measurement is not trivial, however, since the cathode spot diameter is on the order of millimeters and both the cathode surface adjacent to the cathode spot and the arc plasma in front of the cathode surface are very bright. The measurement would thus probably have to be accomplished by magnified imaging of the plasma attachment region immediately in front of the cathode using line filters, or by imaging the brightest area on the cathode surface using neutral density filters. Rapid turn-off of the arc current may also be helpful in accomplishing the latter. 
A direct experimental verification of the thermal nature of the observed stability dependence on the cathode diameter would be desirable for future research. Such a verification could be accomplished in two ways. First, by heating the tip of the cathode whose diameter is slightly larger than the unstable tip diameter by some external means and observing if the instability will occur. Second, by intensively cooling an otherwise unstable cathode and observing if the arc will stabilize. Either way, the experiment would not be an easy one to carry out because of the high thermal load of the cathode $(\sim 1 \mathrm{~kW})$. The first could perhaps be accomplished by heating the cathode with a powerful $(\sim 1 \mathrm{~kW} \mathrm{CW})$ laser; the second - by inserting a short graphite cathode into a water-cooled copper holder. Sufficient direct cooling of the cathode by machining a hollow channel on the inside and forcing a liquid or gas through it is more difficult to achieve technologically, because the unstable cathode diameter is relatively small $(\sim 1 \mathrm{~cm})$ and graphite is relatively fragile. Moreover, the arc would reach the cooling channel within minutes due to cathode erosion. Lastly, an inner cooling channel changes the current distribution in the cathode as well as the cathode temperature, making the thermal dependence of the instability inconclusive in such an experiment.

Another way of affecting the temperature distribution of the cathode for the same cathode diameter is to use a highly oriented graphite crystal as cathode material, as suggested by Prof. Sam Cohen. Such material has high heat conductivity parallel to the crystal planes $(\approx 3,000 \mathrm{~W} / \mathrm{m} \cdot \mathrm{K}$ at $2,000 \mathrm{~K}[76])$ that greatly exceeds heat conductivity of the polycrystalline graphites and carbon that where used in the present experiments $(\approx 40 \mathrm{~W} / \mathrm{m} \cdot \mathrm{K}$ at $2,000 \mathrm{~K}[60])$, and would cool the cathode tip more efficiently. One caveat, however, is that the electrical conductivity is also anisotropic to the same degree as the heat conductivity [60], and might affect the current distribution.

The one-dimensional analytical model was derived under the assumption of small amplitude (Section 3.3.2) and is therefore not applicable for quantitative description of the instability at large amplitude. In future work, it may be desirable to attempt to reproduce the arc shape for large spot motion amplitudes by using a simple "particle code" simulation. The simulation would introduce particles at regular time intervals at a position on the cathode surface (the simulated cathode 
spot) that would vary in time, with the initial particle velocity normal to the cathode surface. The particles are non-interacting, and would be tracked as they follow straight line, ballistic trajectories after being ejected from the "cathode spot". Particles would be removed as they reach a distance from the "cathode" designated as the "anode". As long as the time interval between adjacent particles is sufficiently small compared with the time scale of the cathode spot motion, the particles would track the location of the center of the arc column. If line segments connecting sequential particles are drawn, a time-evolution simulation of the arc column would result.

Besides simulating large amplitude spot motion, the code could also take into account shapes of the naturally eroded cathodes more realistically then the flat cathode surface used in the analytical solution, by appropriately programming the trajectory of the simulated "cathode spot" where the particles are introduced. The code could then be used to simulate the column shapes observed, for example, for large amplitudes on the "paddle" shaped cathode with a convex tip (Figure 4.16). In addition, the length of the simulated arc column could be computed, and the spectrum of its time variation compared to the fluctuation spectrum observed during large amplitude instability.

The simulation could be made to model the self-magnetic field effects as follows. The force on the current in each individual line segment connecting particle pairs due to the currents in all the other line segments could be computed, and the individual particle trajectories adjusted accordingly. However, besides complicating the simulation considerably, the self-field effect simulation would still be a thinwire approximation, and would only be valid for low amplitudes when the radius of curvature of the simulated column is larger then the actual arc column radius.

As with the applied AC magnetic field experiments, the model without the self-magnetic field effects has been used to describe the unstable arc shape. Since the arc shape during low amplitude instability is similar to that in the applied AC transverse magnetic field experiments, the self-field effects are not expected to play a dominant role, as discussed above in Section 5.1. A future resolution of the issue in the applied magnetic field case would then carry over to the instability case as well. 
The origin of the cathode spot motion during arc instability remains open. As mentioned above, interaction of the current distribution in the cathode with the current in the cathode region of the arc remains as the most likely cause of spot displacement. A more quantitative assessment of this interaction is needed, possibly including a calculation of current distribution in the cathode tip and the resulting forces for different locations of the spot on the cathode surface.

As in the arc deflection study, an extrapolation of the spot motion model of instability was made to an industrial-scale arc furnace using the approximate parameters available in the literature. It appears that this type of instability could have a significant effect on the arc shape in this regime. However, as in the arc deflection case, such a large extrapolation requires confirmation on an intermediate level experiment $(\sim 10 \mathrm{kA})$. Such an experiment could look for a systematic dependence of the unstable behavior on cathode geometry, such as, for example, cathode diameter thresholds for instability occurrence. Only the upper threshold on the cathode diameter could be studied at such currents however, since cathodes with diameter on the order of the cathode spot diameter would explode due to high resistive power dissipation. 


\section{Appendix A}

\section{Force due to Self-Magnetic Field of the Arc Column}

An expression for the force per unit length due to self-field for a plane curve $(z, \xi(z))$, for $\xi$ small compared to the length scale of bending is given in [77]:

$$
F_{\text {self }} \approx \frac{\mu_{0} I^{2}}{4 \pi} \int \frac{\left(z-z^{\prime}\right) \frac{\partial \xi\left(z^{\prime}\right)}{\partial z^{\prime}}-\xi(z)-\xi\left(z^{\prime}\right)}{\left[\left(z-z^{\prime}\right)^{2}+a^{2}\right]^{3 / 2}} d z^{\prime}
$$

where $a$ is the arc radius. For a parabolically deflected arc, $\xi=A z^{2}$. Integrating Eq. (A.1) from the cathode $z=0$ to the anode $z=h$, the force per unit length is:

$$
\begin{aligned}
& F_{\text {self }}= \\
& -\frac{\mu_{0} I^{2} A}{4 \pi}\left\{\ln \left(\frac{\sqrt{(h-z)^{2}+a^{2}}+h-z}{\sqrt{z^{2}+a^{2}}-z}\right)-\frac{h-z}{\sqrt{(h-z)^{2}+a^{2}}}-\frac{z}{\sqrt{z^{2}+a^{2}}}\right\}
\end{aligned}
$$

The maximum value of $F_{\text {self }}$ is at $z=h / 2$ and for $a / h \ll 1$ is equal to:

$$
F_{\text {self,max }}=-\frac{\mu_{0} I^{2} A}{4 \pi}\left(2 \ln \frac{h}{a}-2\right)
$$


where the minus sign indicates that the direction of the force is opposite to the deflection $\xi$, and is acting to decrease the deflection. The curvature for the parabola $\xi=A z^{2}$ is $1 / R \approx-\xi^{\prime \prime}(z)=-2 A$, for $\xi^{\prime}(z)=2 A z \ll 1$, i.e. for $z \ll R$. Eq. (A.3) thus becomes

$$
F_{\text {self,max }}=\frac{\mu_{0} I^{2}}{4 \pi R}\left(\ln \frac{h}{a}-1\right)
$$

Comparing the above to Eq. (3.20) and using the definition for $N$, obtain that for low frequency and DC deflection:

$$
N=\frac{\mu_{0} I^{2}}{4 \pi m v^{2}}\left(\ln \frac{h}{a}-1\right)
$$

For higher applied magnetic field frequencies, when the arc shape is not parabolic, integration in Eq. (A.1) has to be done numerically. Substituting the arc shape derived neglecting the self-field effect (Eq. (3.9)) into Eq. (A.1), the resulting $F_{\text {self }}$ into Eq. (3.22), and solving the differential equation numerically, an approximate arc shape with the self-field effect can be obtained. Figure A.1 compares the arc shape thus obtained with the one neglecting the self-field effect. A comparison with Figure 3.8 shows that the analytic form Eq. (3.20) gives an overestimate of the self-field effect. The arc shape in Figure A.1 is still an approximation, however, since the self-field force obtained is not self-consistent and the process needs to be iterated. Also, Eq. (A.1) starts to break down as $\chi(\zeta)$ approaches the wavelength of the bending. 


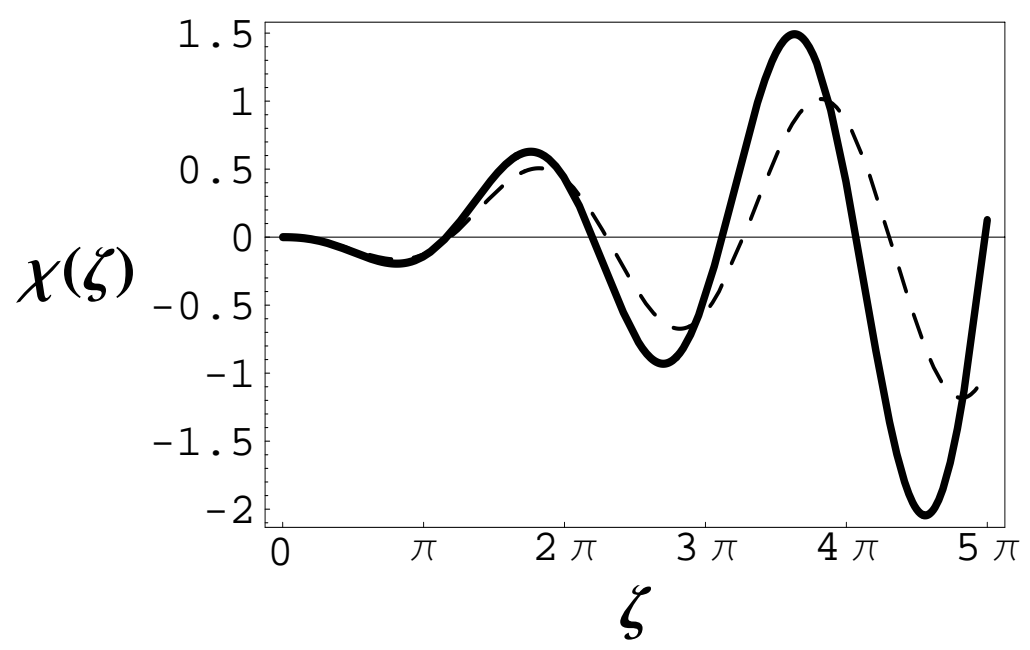

Figure A.1: Numerically calculated arc shape with the self-magnetic field effect. For comparison, the shape calculated without the self-field effect is shown as the dashed curve. 


\section{Appendix B}

\section{Exploratory Study of Arc Instability in Applied Axial Magnetic Field}

This Appendix gives a description of some exploratory experiments on arc stability in an applied magnetic field parallel to the arc column axis.

\section{B.1 Axial Field Experimental Setup}

A pair of the same kind of coils are used for the axial magnetic field as are used for the transverse magnetic field experiments ("L2" coils, Section 2.1.2). The location of axial field coils is shown in Figure B.1. As can be seen in the figure, the coils are mounted directly on top of each other, with the top coil flush with the anode surface. The coils are protected with $1 / 32^{\prime \prime}$ thick aluminum shields on the top and inside surface facing the anode. The coils can also be seen in the picture in Figure 2.3 as the annulus of the same dimensions as the transverse field coils, with the anode in its center and with the $90^{\circ}$ view mirror mounted on top. The coils are connected in series, and powered using either the Techron 7560 Audio Amplifier used in the transverse magnetic field experiments or an HP 6260B 0-100 A DC power supply. The magnetic field at a hight of $3.1 \mathrm{~cm}$ above the non-magnetic steel 


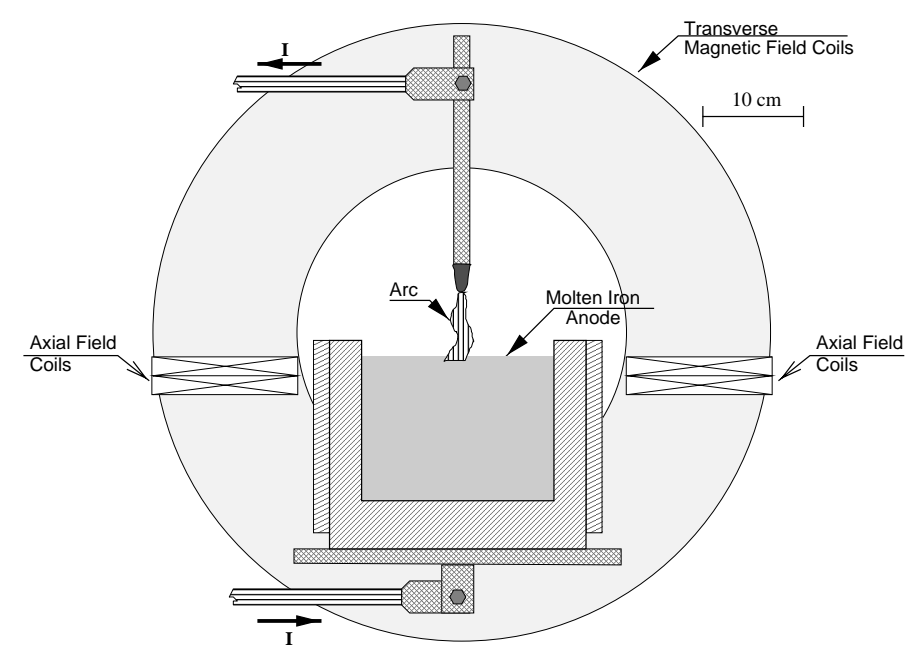

Figure B.1: Experimental setup (side view) showing the location of the axial field coils. The transverse field coils are also shown. The anode shown is the iron anode in the $\mathrm{MgO}$ conducting crucible.

anode surface was measured using a Walker MG-50 gaussmeter to be 2.0 Gauss/A. In the experiments, axial fields applied where 0-50 Gauss and 200 Gauss. The current in the coils is monitored using a Fluke Y1800 DC/AC current meter with a frequency response DC-1 kHz. The experimental setup is otherwise unchanged from that described in Chapter 2. The axial magnetic field coils are not energized during experiments other than described in this Appendix.

\section{B.2 Experimental Results}

When an axial magnetic field is applied with field strength $\geq 10$ Gauss, the arc assumes a helical shape, and produces a high-pitched sound. This instability occurs regardless of the presence of the cathode spot motion instability described in Chapter 4. This has been ascertained by running with both the $3 / 8^{\prime \prime}$ standard cathodes, which can exhibit the spot motion instability, and with the $1 / 2^{\prime \prime}$ cathodes, which are always stable with no external field applied. With the $1 / 2^{\prime \prime}$ cathode runs, the arc became stable as soon as the axial magnetic field was turned off, while with the $3 / 8^{\prime \prime}$ cathode, the arc was often unstable after the axial field was switched off. Runs have been done when both the spot motion and axial field instabilities were 
present. In such cases, arc shape was irregular and the sound produced by the arc was not of a single pitch.

The threshold for occurrence of the instability is approximately 10 Gauss. Between approximately 10 and 30 Gauss, the instability is intermittent, with unstable bursts interspersed by periods of stability. Sample waveforms under such conditions are shown in Figure B.2. Images of the cathode region acquired during the same time period as the waveforms are shown in Figure B.3. As can be seen from the images, the cathode spot was stationary during this period of low amplitude instability.

A sequence of images of somewhat larger amplitude instability for a longer arc is shown in Figure B.4. As can be seen from the sequence, periods when the arc is stable still exist.

Higher applied axial fields lead to steady, relatively large amplitude column oscillations. Sample waveforms for large amplitude oscillation are shown in Figure B.5. As can be seen in the figure, several frequency components are present. At approximately 40 Gauss, the arc typically extinguishes after a few seconds of instability. Higher fields for typical arc lengths lead to almost immediate extinction.

Figure B.6 shows shows the frequencies of column oscillation for a set of runs, as obtained from the collimated photodiode array diagnostic (Section 2.2.5), in the (electrode gap - applied magnetic field strength) parameter space. The frequency is plotted separately vs. the electrode gap and vs. the applied field strength in Figures B.7 and B.8, respectively. As can be seen from the plots, the frequency does not depend on the arc length, but increases approximately linearly with the applied magnetic field strength.

The dependence of the instability frequency on the arc current is less clear from the few data points obtained at constant applied magnetic field (Figure B.9). However, during the run, as the arc current was increased at a fixed axial magnetic field, the frequency of the sound produced by the arc was heard to increase as well. 


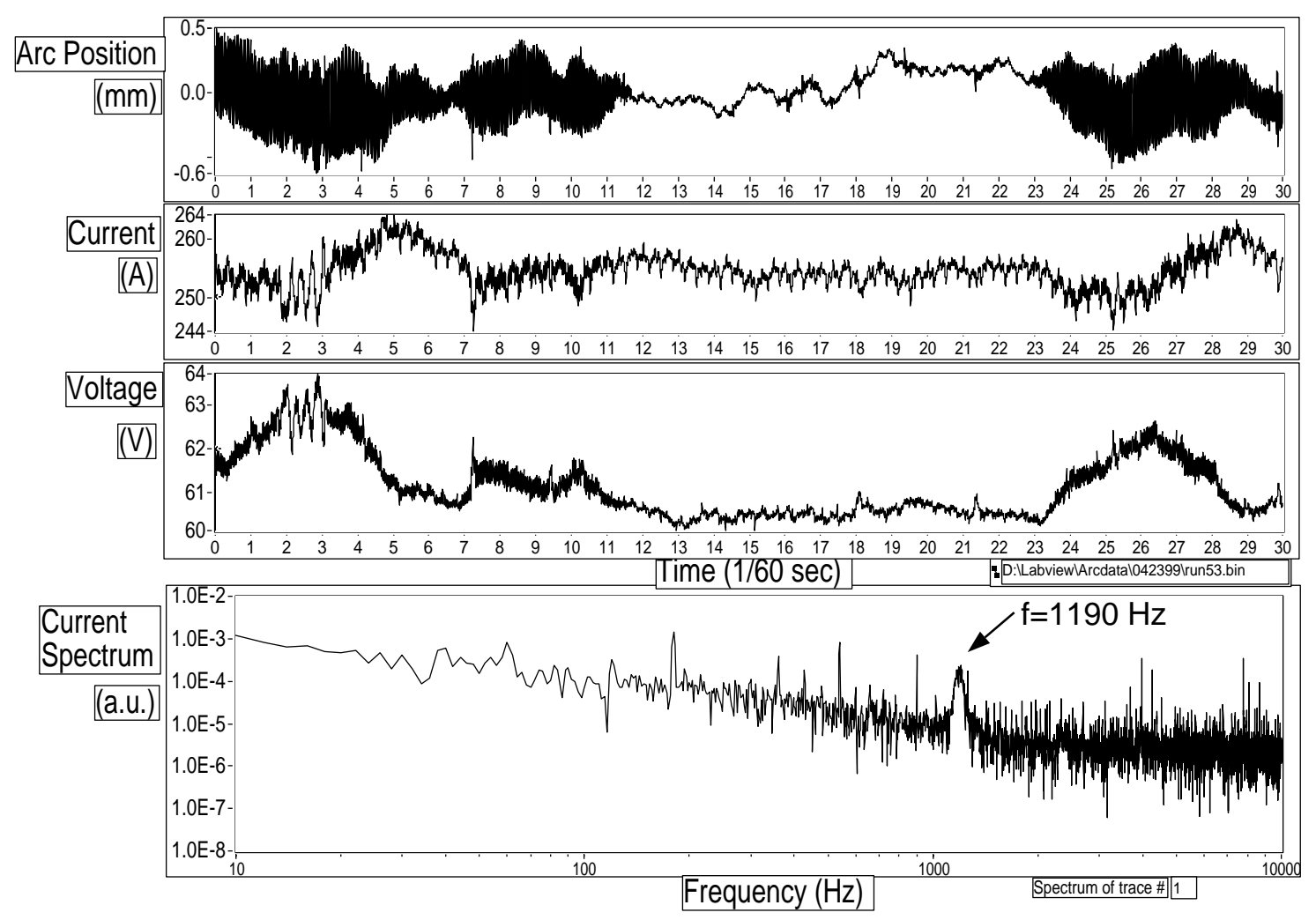

Figure B.2: Waveforms showing bursts of instability during axial magnetic field application. The axial magnetic field is 29 Gauss, average arc current is $254 \mathrm{~A}$, electrode gap is $3.9 \mathrm{~cm}$, the cathode used is $1 / 2 "$ dia. (\#3 in Table 4.1). The frequency of arc oscillation is well defined at $1190 \mathrm{~Hz}$. Unlike the case with arc instability with no external magnetic field, no harmonic of the oscillation frequency is visible on the current spectrum (compare with Figure 4.4). Images of the cathode region taken during the time interval shown here are given in Figure B.3.

Waveforms of the instability in 200 Gauss axial field in an electrode gap of $\approx 3 \mathrm{~mm}$ are shown in Figure B.10. In this case, the magnetic field was set up prior to arc initiation. High pitch sound of instability was heard immediately after the electrodes where separated to a gap of $\approx 3 \mathrm{~mm}$. The single data sample shown was taken, and the arc extinguished less than $10 \mathrm{sec}$ after initiation. Upon examination, it was found that the anode had an $4 \mathrm{~mm}$ deep by $5 \mathrm{~mm}$ dia. hole directly beneath the cathode that has formed during the brief arc operation. 


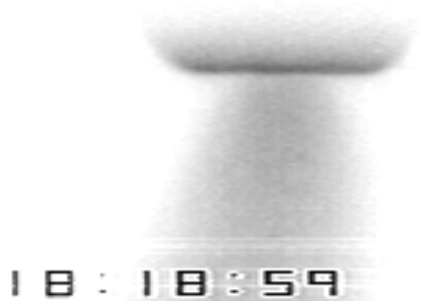

(a)

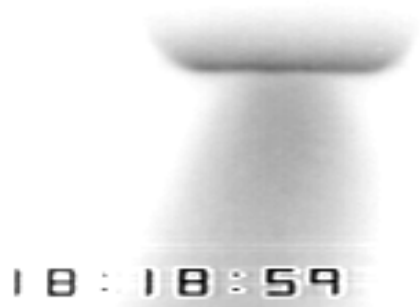

(b)

Figure B.3: Magnified view of the cathode region during instability in axial applied magnetic field shows no cathode spot motion. (a) a single frame with $10 \mu$ sec exposure. (b) the average of 30 consecutive frames, including the one shown in (a), taken over $0.5 \mathrm{sec}$. The average is almost identical to the single frame, indicating that the section of arc in the cathode region (and therefore, the cathode spot) was stationary during the instability. The waveforms corresponding to these 30 frames are given in Figure B.2. The axial magnetic field is 29 Gauss, average arc current is $254 \mathrm{~A}$, electrode gap is $3.9 \mathrm{~cm}$, frequency of column oscillation is $1190 \mathrm{~Hz}$, the cathode used is \#3 in Table 4.1. The horizontal scale is expanded 2.3:1.
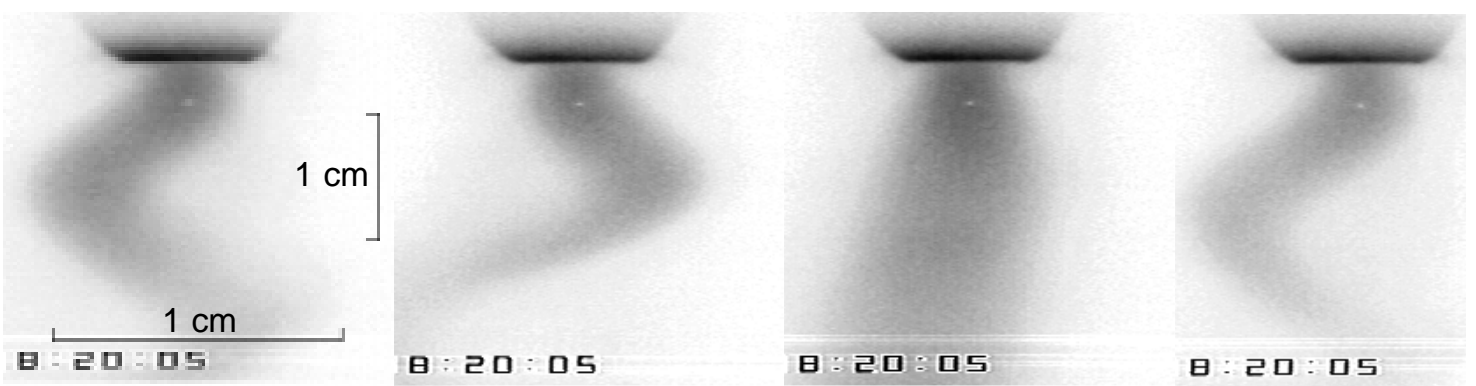

Figure B.4: A sequence of images of instability in axial magnetic field for a relatively long arc (only the upper half the arc is visible at this magnification). Periods of stability still exist (3rd frame from the left). Average arc current is $256 \mathrm{~A}$, axial field is 21 Gauss, electrode gap is $5.3 \mathrm{~cm}$, frequency of column oscillation is $1740 \mathrm{~Hz}$. The horizontal scale is expanded 2.3:1; image contrast is enhanced to emphasize column shape. 


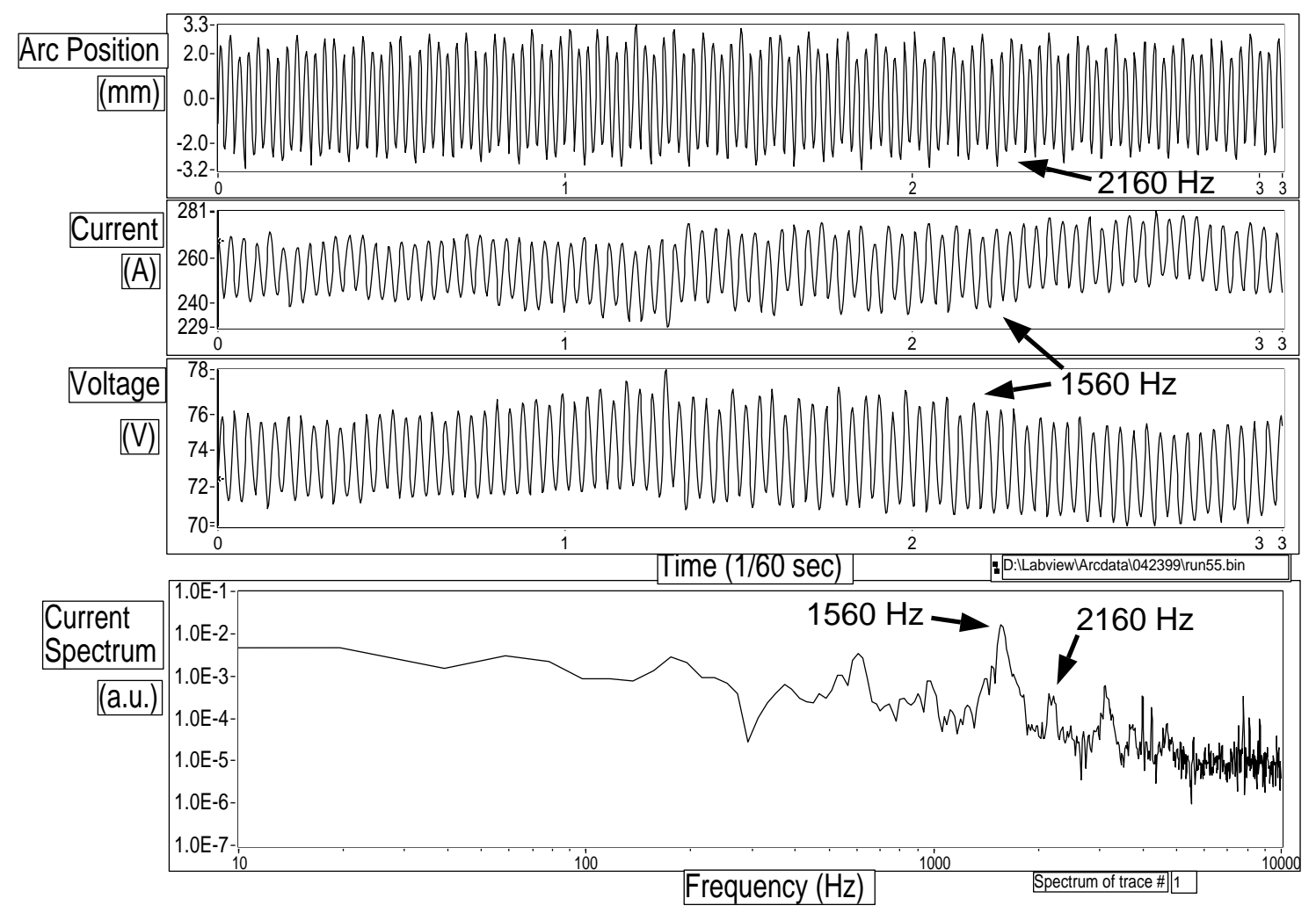

Figure B.5: Waveforms during large amplitude instability. The axial magnetic field is 37 Gauss, average arc current is $255 \mathrm{~A}$, electrode gap is $4.1 \mathrm{~cm}$. Several frequency components are present. The most prominent frequency on the column oscillation is $2160 \mathrm{~Hz}$, on the current and voltage oscillation- $1560 \mathrm{~Hz}$. The $2160 \mathrm{~Hz}$ shows up weakly on the current spectrum. 


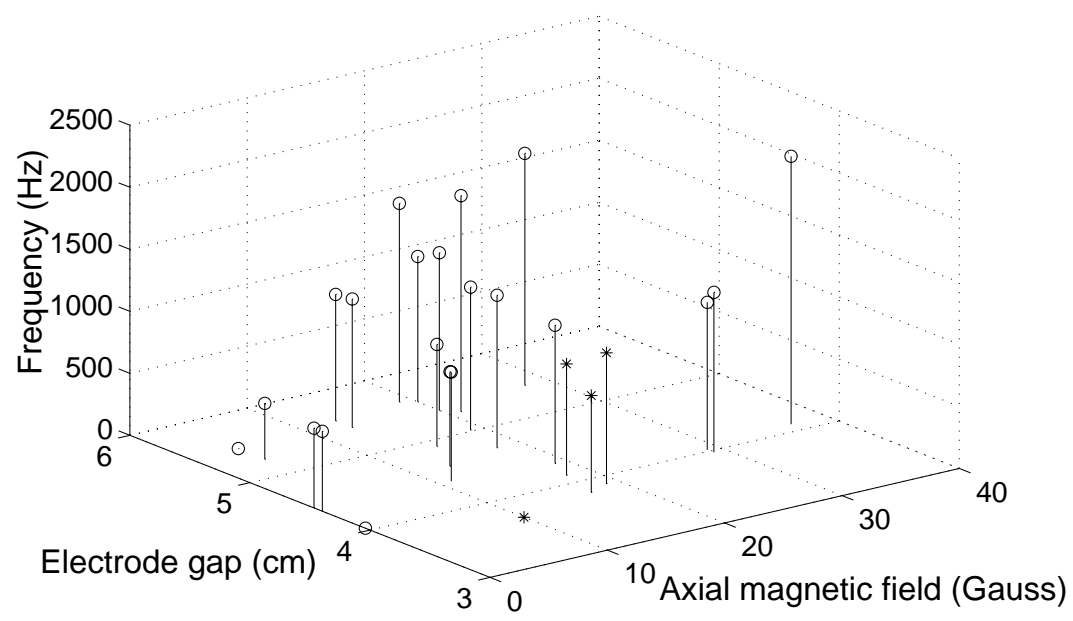

Figure B.6: Frequency of column oscillation in the electrode gap - axial magnetic field parameter space. Arc current is $254 \pm 10 \mathrm{~A}$ for points labeled "o", and $153 \pm 5 \mathrm{~A}$ for "*". Stable runs are indicated as zero frequency.

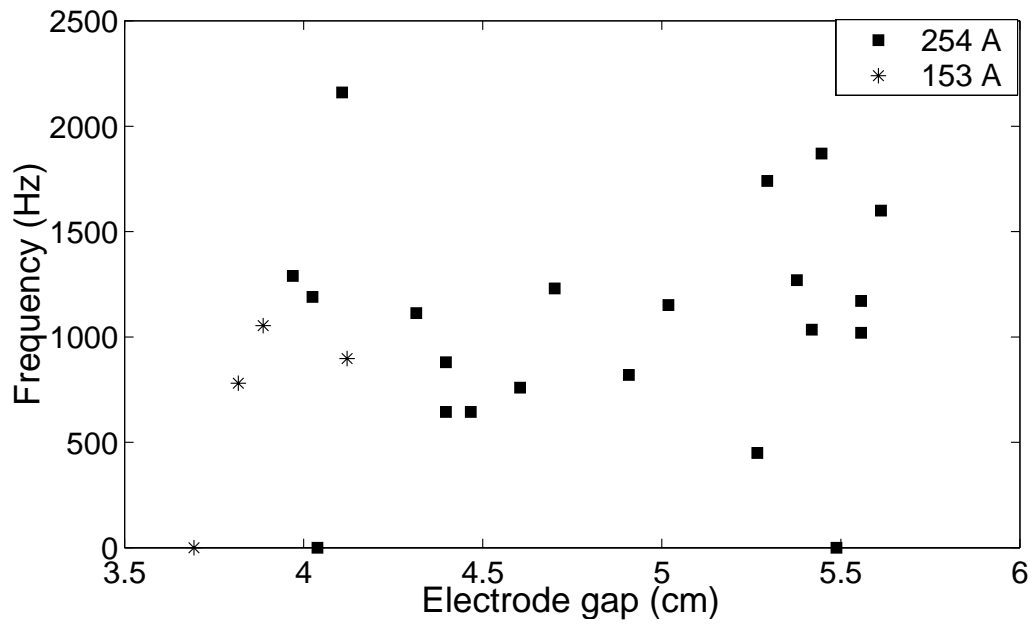

Figure B.7: Frequency of column oscillation vs. the electrode gap for the same set of runs as shown in Figure B.6. No dependence on the gap length can be seen. 


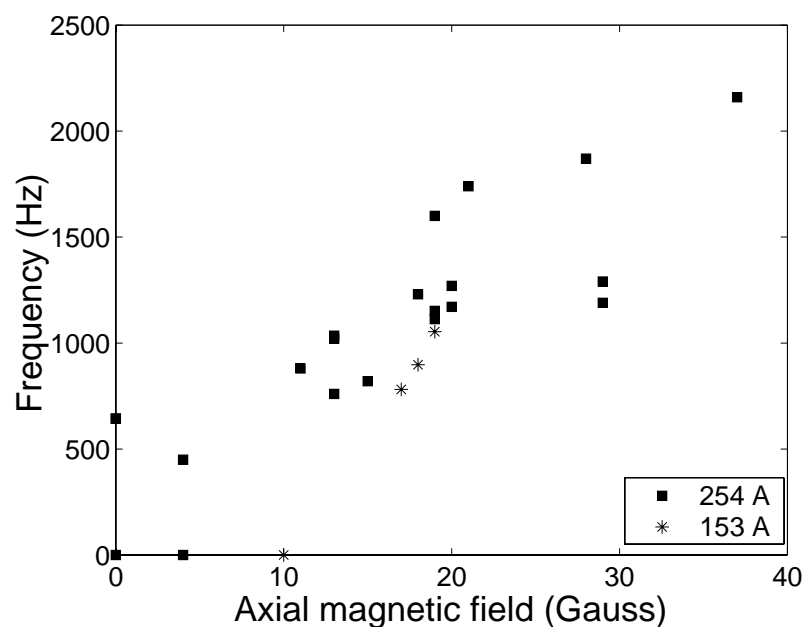

Figure B.8: Frequency of column oscillation vs. the applied axial magnetic field for the same set of runs as shown in Figure B.6. A clear trend of increasing frequency with magnetic field strength can be seen. Stable runs are indicated as zero frequency.

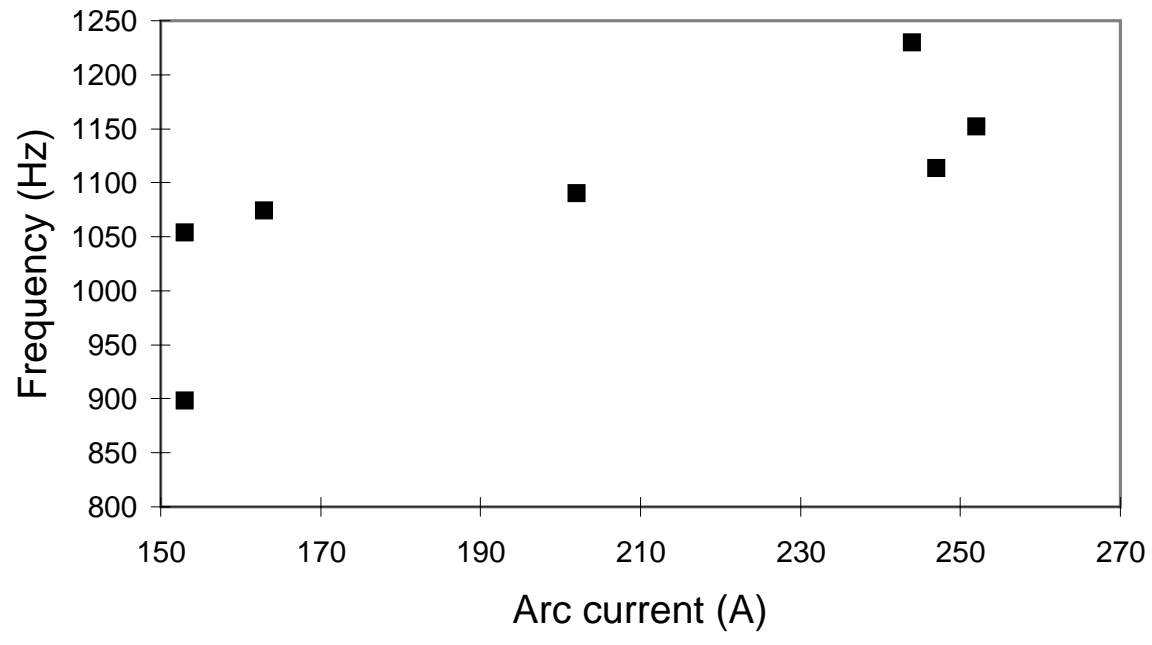

Figure B.9: Frequency of column oscillation vs. arc current for a subset of runs shown in Figure B.6. The applied magnetic field is 18-19 Gauss. The electrode gap is $3.9-5.0 \mathrm{~cm}$. The frequency is weakly dependent on the arc current in this range. 

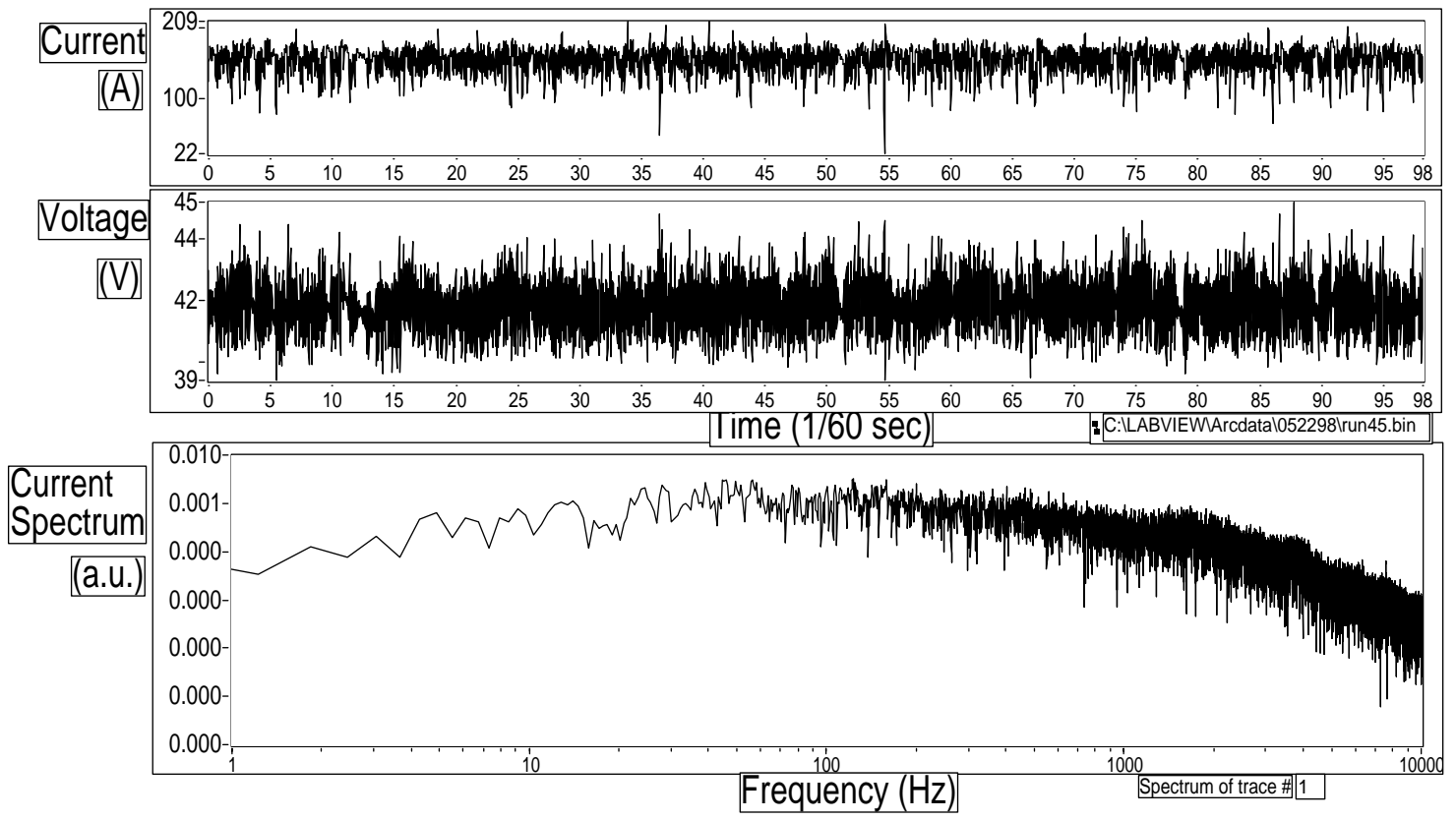

Figure B.10: Waveforms during instability in 200 Gauss axial magnetic field. The electrode gap is approximately $3 \mathrm{~mm}$, average arc current is $153 \mathrm{~A}$. The fluctuation spectrum is very broad, with high frequency components $(>1 \mathrm{kHz})$ present. 


\section{B.3 Speculation on the Mechanism of Instability in Axial Field}

Occurrence of helical arc instability in applied axial magnetic field has been studied previously, as discussed in Section 1.4.2. However, the studies concentrated on lower current ( $\leq 120 \mathrm{~A})$ and wall-stabilized arcs (Ref. 22-25). Ref. 52 describes application of the helically shaped arc to arc heaters, in which an oscillating axial magnetic field caused the arc helix to expand and contract radially, increasing the interaction volume between the arc and the gas to be heated.

Experiments with liquid metal described in Ref. 78 show kink instabilities on a free-falling current-carrying mercury jet in axial magnetic field. In Ref. 79, stability of a fluid of finite conductivity in axial magnetic field without flow is examined theoretically and applied to the experiments of Ref. 78.

Wang et al. [26] performed a theoretical investigation of a free-burning arc in applied axial field without the electrode jet effects. As described in Section 1.4.2, experiments by Bellan et al. [31] to stabilize arc blowout by applying an axial magnetic field yielded helical arc instability as a side effect.

The mechanism of the instability in the present experiments may be as follows. Since the collision frequency is much higher than the ion gyrofrequency (Table 1.1), the arc plasma is not magnetized by the applied magnetic field, and is thus freely transported across the magnetic field lines. Moreover, magnetic field is not "frozen in" the plasma, since the magnetic Reynolds number is at most $\sim 10^{-3}$ (for the 200 Gauss applied field experiment). The axial magnetic field thus provides no stabilizing effect for the arc, as opposed to high temperature plasma devices such as a z-pinch or a tokamak, where sufficiently strong axial or toroidal, respectively, magnetic field stabilizes kink modes [46].

On the contrary, the axial field is destabilizing, since a right-handed (when the magnetic field direction is from the anode to the cathode) or left-handed (when the magnetic field direction is from the cathode to the anode) helical perturbation results in radially outward component of the $\vec{J} \times \vec{B}$ force, which further expands the helix, as can be seen in the schematic shown in Figure B.11. Since the radius 


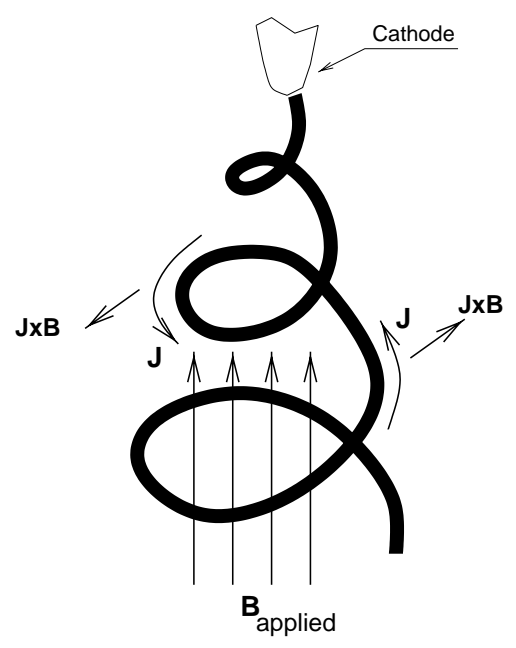

Figure B.11: A schematic illustrating the forces tending to expand and rotate the right-handed helical arc column in applied axial magnetic field for the case of the magnetic field directed from the anode to the cathode. In the case of the opposite magnetic field direction, the unstable helix would be left-handed. The schematic is not to scale.

of the helix is growing with distance from the cathode, a radial component of the arc current is present. This radial component produces an azimuthal component of the $\vec{J} \times \vec{B}$ force, tending to rotate the column in the right-hand sense w.r.t. the applied magnetic field direction.

The cathode jet tends to convectively stabilize the expansion of the helix. For a finite arc length, once the helical perturbation has formed, the helix has only a finite time in which to expand due to the $\vec{J} \times \vec{B}$ force - the transit time of the jet across the gap between the electrodes.

The frequency of column motion as observed by the collimated photodiode array (Section 2.2.5) is interpreted as being determined by the wavelength of the helix $\lambda$ and the speed at which the helix is moving across the horizontal line of sight of the array, i.e. the jet speed $v$ :

$$
f=v / \lambda
$$

This relationship is confirmed by the images of the unstable arc, such as shown in Figure B.4. The wavelength in the images is approximately $2 \mathrm{~cm}$. The average 
jet speed expected at this arc current (256 A) from the transverse magnetic field experiments is approximately $33 \mathrm{~m} / \mathrm{s}$ (Figure 3.17). According to Eq. (B.1), this implies a frequency of $1650 \mathrm{~Hz}$, in fair agreement with the measured frequency of $1740 \mathrm{~Hz}$.

The wavelength of the helix is expected to be the wavelength with the highest growth rate. According to Ref. 26, the most unstable modes are those with the wavelength $\lambda \sim 2 \pi r$, where $r$ is the conducting radius of the arc column. In the present experiments, $r \approx 3 \mathrm{~mm}$, giving the expected wavelength of $2 \pi(0.3 \mathrm{~cm}) \approx$ $2 \mathrm{~cm}$, in agreement with the observations.

The weak dependence of the frequency on the arc current seen in Figure B.9 can be explained on the basis of the above interpretation as follows. The jet speed has been found to scale linearly with the arc current $I$ from the transverse magnetic field experiments (Figure 3.17): $v \propto I$. The radius of the arc column is expected to scale as $r \propto \sqrt{I}$ if the current density in the column remains constant. The frequency would therefore be related to arc current as:

$$
f=v / \lambda \sim \frac{v}{2 \pi r} \propto \frac{I}{\sqrt{I}} \propto \sqrt{I}
$$

Thus the frequency would be expected to scale approximately as $\sqrt{I}$, not unlike what is observed in Figure B.9.

Future research on the axial magnetic field instability should verify that the sense of the helix is in fact right-handed when the magnetic field direction is from the anode to the cathode, and left-handed when the magnetic field is in the opposite direction. Additionally, a theoretical explanation of the dependence of wavelength of the instability on the applied field strength is needed. Relation (B.1) together with the data in Figure B.8 imply that the wavelength of the instability is inversely proportional to the applied magnetic field. 


\section{Bibliography}

[1] P. Fauchais and A. Vardelle. Thermal plasmas. IEEE Trans. Plas. Sci., 25(6):1258-80, Dec. 1997.

[2] Ben Bowman. Properties of arcs in DC furnaces. In Electric Furnace Conference Proceedings, volume 52, pages 111-120. Iron \& Steel Society, 1994.

[3] J. F. Lancaster, editor. The Physics of Welding. Pergamon Press, 2nd edition, 1986.

[4] International Iron and Steel Institute. World crude steel production by process, 1998. Iron E3 Steelmaker, 26(13):4-5, July 1999.

[5] N. Ao and M. Nakai. Prevention of arc deflection and electro-magnetic stirring in DC arc furnaces. SEAISI Quarterly, pages 20-29, Oct. 1994. South East Asia Iron and Steel Institute.

[6] B. Bowman and F. Fitzgerald. Hot spots in arc furnaces. J. Iron and Steel Institute, pages 178-186, March 1973.

[7] Masoud Sharifi. Magnetic field modeling of a direct current electric arc furnace. Master's thesis, University of Toronto, 1994.

[8] E. Pfender. Electric arcs and arc gas heaters. In Merele N. Hirsh and H. J. Oskam, editors, Gaseous Electronics, volume I: Electrical Discharges, chapter 5. Academic Press, New York, 1978.

[9] Yuri P. Raizer. Gas Discharge Physics. Springer-Verlag, New York, 1991.

[10] G. R. Jones. High Pressure Arcs in Industrial Devices. Diagnostic and Monitoring Techniques. Cambridge University Press, Cambridge, 1988. 
[11] Wolfgang Finkelnburg. The High Current Carbon Arc. Dept. of Commerce, Washington, DC., 1947. FIAT Final Report 1052-PB-81644.

[12] W. Finkelnburg and H. Maecker. Electrische Bögen und thermisches Plasma, volume 22 of Encyclopaedia of Physics, page 254. Springer Verlag, 1956.

[13] Max F. Hoyaux. Arc Physics. Springer-Verlag, New York, 1968.

[14] P. M. Bellan. Vorticity model of flow driven by purely poloidal currents. Phys. Rev. Lett., 69(24):3515-18, Dec. 1992.

[15] H. Maecker. Plasmaströmungen in lichtbögen infolge eigenmagnetischer kompression. Z. Phys., 141:198, 1955.

[16] T. W. Myers and W. C. Roman. Survey of investigations of electric arc interactions with magnetic and aerodynamic fields. Technical Report ARL 66-0184, Aerospace Research Labs, Wright-Patterson Airforce Base, September 1966.

[17] H. Maecker. Principles of arc motion and displacement. Proc. IEEE, 59(4):439449, April 1971.

[18] K. Bartels and J. Uhlenbusch. Bewegung eines freibrennenden lichtbogens in einem oszillierenden transversalen magnetfeld. Z. angew. Physik, 29(2):122$125,1970$.

[19] Zeng-Yuan Guo. The temperature and flow field of a free burning arc deflected by a transverse magnetic field. Int. J. Heat Mass Transfer, 27(3):383-390, 1983.

[20] G. Speckhofer and H. P. Schmidt. Experimental and theoretical investigation of high-pressure arcs-part II: The magnetically deflected arc (three-dimensional modeling). IEEE Trans. Plasm. Sci., 24(4):1239-1248, August 1996.

[21] G. B. Serdyuk. Calculations for welding arcs in transverse magnetic fields. Automatic Welding, 13(11):26-34, 1960.

[22] H. G. Hülsmann and J. Mentel. The helical magnetic instability of arcs in an axial magnetic field treated by a linear time dependent perturbation theory. Phys. Fluids, 30(7):2266-73, Jul 1987. 
[23] H. G. Hülsmann and J. Mentel. Experimental investigation of the helical magnetic instability of an arc discharge in an axial magnetic field and comparison with theory. Phys. Fluids, 30(7):2274-9, Jul 1987.

[24] P. J. Gaede. Der wendelnde wasserstoffbogen. Z. Physik, 255:40-58, 1972.

[25] K. A. Ernst and J. Kopainsky. Der einsatz def wendelinstabilität in wandstabilisierten lichtbögen. Z. Physik, 265:253-65, 1973.

[26] Xiaogang Wang, Jinyuan Liu, Ye Gong, Guobing Li, and Tengcai Ma. An electrostatic magnetohydrodynamics theory for resistive-viscous helical instabilities of arc discharges. Phys. Plasmas, 4(8):2791-7, Aug 1997.

[27] R. E. Blundell, Michael T. C. Fang, and A. Vourdas. Stability of a DC SF 6 arc in an axially accelerating flow. IEEE Trans. Plasma Sci., 25(5):852-9, Oct. 1997.

[28] A. M. Howatson and D. R. Topham. The instability of electric arcs burning axially in accelerated flow. J. Phys. D: Appl. Phys., 9:1101-9, 1976.

[29] K. Ragaller, U. Kogelschatz, and W. R. Schneider. über die wendel-instabilität eines konvektionsstabilisierten hochstrom-hochdruck-lichtbogens. Z. Naturforsch., 28a:1321-28, 1973.

[30] K. Ragaller. Investigations on instabilities of electric arcs. Z. Naturforsch., 29a:556-567, 1974.

[31] Paul M. Bellan and Jay W. Higley. Magnetic suppression of arc blowout in a model arc furnace. IEEE Trans. Plasma Sci., 20(6):1026-35, December 1992.

[32] B. Bowman and G.R. Jordan. The magnetic stability of furnace arcs. In IEE Conference on Gas Discharges, pages 231-235, London, 1970. Institution of Electrical Engineers.

[33] Von Siegbert Witkowski. Das wendeln des hochstromkohlebogens. Z. angew. Physik, 11(4):135-142, 1958.

[34] R. W. Montgomery and C. M. H. Sharp. The effect of cathode geometry on the stability of arcs. Brit. J. Appl. Phys.(J. Phys. D), 2(2):1345-8, 1969. 
[35] H. Edels. Properties of the high pressure ultra high current arc. In L. Pekarek and L. Laska, editors, 11th International Conference on Phenomena in Ionized Gases, volume 1, pages 9-59, Prague, 1973.

[36] S.-E. Stenkvist. Single electrode d-c arc furnace. Iron and Steel Engineer, pages 50-54, May 1985.

[37] Peter Bonanos. Coil data compilation. Technical Report 207, Princeton Plasma Physics Lab, December 1964.

[38] American National Standards Institute, Inc. Practice for Occupational and Educational Eye and Face Protection. American Society of Safety Engineers, 1989.

[39] David Book. NRL Plasma Formulary. Naval Research Lab, 1990.

[40] H. Maecker. Z. Physik, 136:199, 1953.

[41] Maher I. Boulos, Pierre Fauchais, and Emil Pfender. Thermal Plasmas: Fundamentals and Applications, volume I. Plenum Press, 1994.

[42] H. Maecker and H. G. Stablein. What keeps and arc standing in a cross flow? IEEE Trans. Plasm. Sci., PS-14(4):291-299, August 1986.

[43] D. J. Tritton. Physical Fluid Dynamics. Oxford University Press, 2nd edition, 1988.

[44] A. Jeffrey and T. Taniuti. Magnetohydrodynamic Stability and Thermonuclear Containment. Academic Press, 1966.

[45] F. B. Hildebrand. Advanced Calculus for Applications. Prentice-Hall, 1962.

[46] J. P. Freidberg. Ideal Magnetohydrodynamics. Plenum Press, 1987.

[47] Abelbeck Software. KaleidaGraph ${ }^{\mathrm{TM}}$. Software package, Ver. 3.0.1, 1993.

[48] E. Pfender, J. Fincke, and R. Spores. Entrainment of cold gas into thermal plasma jets. Plasm. Chem. Plasm. Proces., 11(4):529-543, 1991.

[49] R. J. Maqueda and G. A. Wurden. Fast imaging of visible phenomena in TFTR. Nucl. Fusion, 39(5):629-636, May 1999. 
[50] J. C. Borrel, L. Mineau, N. Meysoson, and J. L. Roth. Control of the arc deviation in the dc electric arc furnace of Unimetal Gandrange. La Revue de Metallurgie-CIT, 93(4):497, April 1996.

[51] J. W. McKelliget and J. Szekely. A mathematical model of the cathode region of a high intensity carbon arc. J. Phys. D: Appl. Phys., 16:1007-22, 1983.

[52] V. F. Put'ko. Electric arc behavior in dynamic magnetic fields. In O. P. Solonenko and M. F. Zhukov, editors, Thermal Plasma and New Materials Technology, volume 1: Investigations and Design of Thermal Plasma Generators, pages 229-249. Cambridge Interscience Publishing, 1994.

[53] Peter Plaschko. Helical instabilities of slowly divergent jets. J. Fluid Mech., 92(Part 2):209-215, 1979.

[54] S. C. Crow and F. H. Champagne. Orderly structure in jet turbulence. J. Fluid Mech., 48(Part 3):547-591, 1971.

[55] A. Achterberg. The influence of buoyancy on the stability of jets. Astronomy and Astrophysics, 114:233-37, 1982.

[56] Nicholas A. Krall and Alvin W. Trivelpiece. Principles of Plasma Physics. San Francisco Press, 1986.

[57] J. M. Lafferty, editor. Vacuum Arcs. Theory and Application. Wiley \& Sons, 1980 .

[58] I. G. Kesaev. Cathode Processes in Electric Arcs. Sandia Laboratories, 1978. Translation from Russian.

[59] A. E. Guile. Arc-electrode phenomena. Proc. IEE, IEE Reviews, 118(9R):113154, Sep. 1971.

[60] N. S. Rasor and J. D. McClelland. Thermal properties of graphite, molybdenum and tantalum to their destruction temperatures. Phys. Chem. Solids., $15: 17-26,1960$.

[61] M. F. Zhukov, A. C. Koroteev, and B. A. Urukov. Prikladnaya Dinamika Termicheskoii Plasmy. Nauka, 1975. 
[62] G. Ecker. Electrode components of the arc discharge. In Ergebnisse der exakten Naturwissenschaften, volume 33, pages 1-104. Springer-Verlag, 1961.

[63] T. H. Lee, A. N. Greenwood, and W. D. Breingan. A self consistent model for the cathode region of a high pressure arc. In B. Perovic and D. Tosic, editors, Proc. Seventh Int. Conf. Phenom. Ionized Gases, volume 1, pages 670-680, Beograd, Aug. 1965. Gradevinska Knjiga Publishing House. 1966.

[64] J. J. Lowke, P. Kovitya, and H. P. Schmidt. Theory of free-burning arc columns including the influence of the cathode. J. Phys. D: Appl. Phys., 25:1600-6, 1992.

[65] Charles L. Mantell. Carbon and Graphite Handbook. Interscience Publishers, 1968.

[66] Günter Ecker. Theoretical aspects of the vacuum arc. In J. M. Lafferty, editor, Vacuum Arcs. Theory and Application, chapter 7. Wiley \& Sons, 1980.

[67] E. Hantzsche. Thermal runaway prevention in arc spots. IEEE Trans. Plasma Sci., PS-11(3):115-122, Sept. 1983.

[68] Von P. Guillery. Über temperatur und stromdichte an der kathode von hochstromkohlebögen. Z. Naturforschg., 10a:248-9, 1955.

[69] B. Gebhart. Heat Transfer. McGraw-Hill, 2 edition, 1971.

[70] Max Karasik. Driven Motion and Instability of an Atmospheric Pressure Arc. PhD thesis, Princeton University, Jan. 2000.

[71] Ragnar Holm. Electric Contacts Handbook. Springer-Verlag, 3rd edition, 1958.

[72] D. E. Roberts. Spectroscopic investigation of the $10 \mathrm{kA}$ free burning arc in air. Technical Report ULAP-T8, University of Liverpool, March 1972.

[73] J. Mentel. The influence of vaporization upon the roots of a high current arc. Appl. Phys., 15:179-183, 1978.

[74] Doughlas H. McQueen. Noise from electric arc furnaces. I. General considerations. Scandinavian J. Metallurgy, 7:5-10, 1978. 
[75] G. R. Jordan, B. Bowman, and D. Wakelam. Electric and photographic measurements of high-power arcs. J. Phys. D: Appl. Phys., 3:1089-99, 1970.

[76] D. R. Lide and H. P. R. Frederikse, editors. CRC Handbook of Chemistry and Physics. CRC Press, Boca Raton, 75th edition, 1994.

[77] W. B. Thompson. An Introduction to Plasma Physics. Pergamon Press, 1962.

[78] A. Dattner. Current-induced instabilities of a mercury jet. Arkiv för Fysik, 21(7):71-80, 1962.

[79] G. S. Murty. Instability of a conducting fluid cylinder in the presence of an axial current, a longitudinal magnetic field and a coaxial conducting cylinder. Arkiv för Fysik, 19(35):483-97, 1961. 